\title{
Systems Analysis of the Cardiac Myocyte Hypertrophy Signaling Network
}

\author{
A Dissertation \\ Presented to \\ the faculty of the School of Engineering and Applied Science \\ University of Virginia.
}

\author{
In partial fulfillment \\ of the requirements for the degree \\ Doctor of Philosophy in Biomedical Engineering
}

Karen Ann Ryall

May 2014 


\section{Approval Sheet}

This dissertation is submitted in partial fulfillment of the requirements

For the degree of

Doctor of Philosophy in Biomedical Engineering

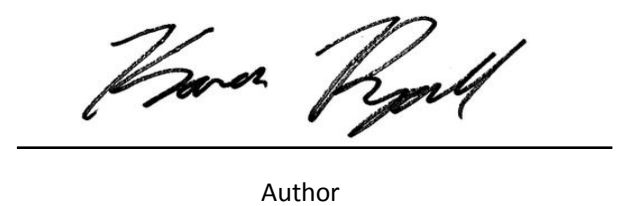

This dissertation has been read and approved by the examining committee:

\begin{tabular}{c} 
Jeffrey J. Saucerman, Ph.D. \\
Dissertation Advisor \\
Brent A. French, Ph.D. \\
Committee Chair \\
Jeffrey W. Holmes, Ph.D. \\
David L. Brautigan, Ph.D. \\
\hline Zhen Yan, Ph.D.
\end{tabular}

Accepted for the School of Engineering and Applied Science:

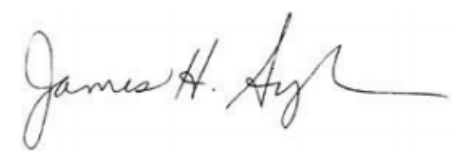

Dean, School of Engineering and Applied Science

May 2014

$\sim \mathrm{ii} \sim$ 
In loving memory of my Dad, Henry Carroll Ryall, who first taught me to love learning and science.

"To the King of ages, immortal, invisible, the only God, be honor and glory forever and ever. Amen."

(1 Timothy 1:17)

$\sim \mathrm{iii} \sim$ 


\section{Acknowledgements}

Graduate school has been a pretty long and overall awesome journey. I am so thankful that I can honestly say I enjoyed graduate school. Yes, there were some tough moments, some 'real' moments, but overall I had fun. I feel privileged to get to say that learning, discovery, and problem-solving are my jobs. I am very thankful for the people who helped get me to this point and helped me have such a positive experience:

Thank you God for loving me and for giving me more blessings, answers, and victories than I deserved. Thank you for creating an amazing world full of surprises and mysteries to discover. Jesus, thank you for giving me self-worth that can survive a million failures.

Thank you Jeff Saucerman for being an outstanding advisor. I think you've ruined me for any future bosses! Throughout grad school you always treated us with kindness, respect, patience, and optimism. You let me decide future directions of my project and gave me nudges when I was scared of taking a risk. You helped me slow down and think when I needed to and cared about my future more than I did. I can't imagine grad school being as positive of an experience as it was without your support and guidance. I am very thankful I ended up with you.

Additional thanks to the rest of my committee: Dr. Jeff Holmes, Dr. Brent French, Dr. David Brautigan, and Dr. Zhen Yan. You all made me come away from committee meetings feeling more jazzed about science than I was before. Your love for what you do is contagious. I came away from my proposal thinking I wanted to do this forever, which I don't think is normal. Thank you for the advice and support over the years. I know my projects were greatly improved by your feedback.

I would also like to thank my lab mates past and present. Special thanks go out to Renata Polanowska-Grabow and Lindsay McClellan for doing the myocyte isolations for the lab. Seriously, I would have "Mastered out" if I had to do them. You are stronger women than I am. I would also like to 
thank Jason Yang, for helping me get started, Robert Amanfu, for many interesting debates, food smells, and equipment fixes over the years, and Eric Greenwald, for many brain storming sessions and thoughtful questions related to planning my work. Additionally, thank you to the following undergraduate students for your contributions to my work over the years: Ashwin Katikapalli, Audrey Parker, David Holland, Matthew Kraeutler, and Kyle Delaney.

I also need to thank several graduate students in other labs for their project advice and support, most notably Bryan Piras. He answered so many questions over the years to fill in the gaps in my knowledge on genetic engineering and molecular biology. He was also one of my first stops if I needed a break. It was a BLAST working with you (get it?). Additionally, thank you Team "Research and Destroy" Cynthia Huang, Amy Clobes, Katy Parker, Nathan Thomas, and Rachel Evans for being great friends in the department. I would not have been nearly as productive without you guys to encourage and refresh me.

Finally, thank you to my Mom and Dad for doing so much to get me here. You guys taught me about time management, responsibility, and working hard. You taught me to always do my best, even if it goes unnoticed or unrewarded. Thank you for paying for my ridiculously expensive college education Debt + grad school would have really stunk. Special thanks to my Dad for teaching me my first lessons in science and for encouraging me to follow my dreams. Even when my dream was to be an entomologist, you were out in the woods with me looking for weird bugs to capture. I will remember your lessons forever. I wish you could have been here with me at the finish line.

Karen Ann Ryall May 2014

This work has been made possible by generous support from the National Institutes of Health (grant HL094476 to Jeffrey Saucerman, training grant HL007284 supporting Karen Ryall) and the National Science Foundation (pre-doctoral fellowship to Karen Ryall, CAREER grant \#1252854 to Jeffrey Saucerman). 


\section{Table of Contents}

$\begin{array}{ll}\text { Abstract } & 1\end{array}$

List of Figures $\quad 4$

List of Abbreviations 6

CHAPTER 1. Background and significance

1.1 Cardiac hypertrophy is a leading predictor of heart failure 8

1.2 Unique signaling in pathological and physiological hypertrophy 9

$\begin{array}{ll}1.3 \text { Unique growth responses to different stress types } & 9\end{array}$

$\begin{array}{ll}1.4 \text { Network complexity motivates use of systems methods } & 11\end{array}$

$\begin{array}{ll}1.5 \text { Significance } & 12\end{array}$

$\begin{array}{ll}1.6 \text { Specific Aims } & 13\end{array}$

CHAPTER 2. Automated imaging reveals a concentration dependent delay in reversibility of cardiac myocyte hypertrophy

2.1 Introduction 17

$\begin{array}{ll}2.2 \text { Methods } & 18\end{array}$

$\begin{array}{ll}2.3 \text { Results } & 26\end{array}$

$\begin{array}{ll}2.4 \text { Discussion } & 47\end{array}$

CHAPTER 3. Network reconstruction and systems analysis of cardiac myocyte hypertrophy signaling.

3.1 Introduction $\quad 52$

3.2 Methods $\quad 53$

$\begin{array}{ll}3.3 \text { Results } & 58\end{array}$

$\begin{array}{ll}3.4 \text { Discussion } & 81\end{array}$

CHAPTER 4. Phenotypic screen identifies distinct signatures of cardiac myocyte hypertrophy.

4.1 Introduction $\quad 85$

$\begin{array}{ll}4.2 \text { Methods } & 86\end{array}$

4.3 Results $\quad 92$

$\begin{array}{ll}4.4 \text { Discussion } & 123\end{array}$

CHAPTER 5. Reverse Phase Protein Array proteomics analysis of hypertrophic agonists.

$\begin{array}{ll}5.1 \text { Introduction } & 128\end{array}$

$\begin{array}{ll}5.2 \text { Methods } & 128\end{array}$

$\begin{array}{ll}5.3 \text { Results and Discussion } & 129\end{array}$ 
CHAPTER 6. Dissertation conclusions and future directions

$\begin{array}{lr}6.1 \text { Conclusions } & 140\end{array}$

6.2 Future Directions 142

APPENDIX A. Detailed methods for high-throughput cardiac myocyte 149 image acquisition and analysis

$\begin{array}{ll}\text { APPENDIX B. PE ligand trapping model } & 158\end{array}$

APPENDIX C. Hypertrophy signaling network model species and reaction list 163

APPENDIX D. References for experimental validation of transcription 172

factor activities and phenotypic outputs

$\begin{array}{ll}\text { APPENDIX E. CITED4 and myocyte elongation model } & 178\end{array}$

$\begin{array}{lr}\text { References } & 181\end{array}$

$\begin{array}{lr}\text { Curriculum Vitae } & 193\end{array}$ 


\begin{abstract}
Cardiac hypertrophy, the enlargement of myocytes in the heart, develops in response to physiological (e.g. exercise and pregnancy) or pathological (e.g. myocardial infarction and hypertension) stresses, increasing risk of heart failure and malignant arrhythmia. The cardiac hypertrophy response is managed by a dense web of signaling pathways, with many molecular species influencing cardiac myocyte growth. Little is known about the specific signaling pathways that distinguish pathological and physiological forms of hypertrophy. The complexity of this network has hindered the development of successful therapeutic strategies and indicates the need for integrative systems approaches which can provide a global view of functional relationships in the network. The overall goal of this dissertation is to integrate experimental and computational approaches to determine how the components and network topology of hypertrophy signaling lead to differential regulation of myocyte shape and gene expression.
\end{abstract}

To understand network organization in a complex process like hypertrophy, new large scale experimental approaches are required to quantitatively characterize a large number of input and output relationships at multiple time points. To address this challenge, we developed an automated image acquisition method that records $5 \times 5$ mosaic images of fluorescent protein-labeled cardiac myocytes within each well of a 96-well plate using an automated stage and focus. Post-processing algorithms automatically identify cell edges, quantify cell phenotypes, and track cells. We uniquely applied our imaging platform to study hypertrophy reversibility in a scalable cell model. Cell area changes after washout of a dose response to the $\alpha$-adrenergic receptor ( $\alpha A R$ ) agonist phenylephrine (PE) showed that hypertrophy reverses at low but not high levels of $\alpha$-adrenergic signaling: a reversibility delay. Perturbations with specialized $\alpha A R$ antagonists, a mathematical model, and live imaging of $\alpha A R$ localization identify the mechanism for this reversibility delay: ligand trapping with internalized PE acting on intracellular $\alpha A R$ 's. 
While many proteins and genes have been identified that affect hypertrophy, it is unclear how these parts work together as a coordinated system. To address this challenge, we developed a computational model of the cardiac myocyte hypertrophy signaling network to determine how the components and network topology lead to differential regulation of transcription factors, gene expression, and myocyte size. Our computational model of the hypertrophy signaling network contains 106 species and 193 reactions, integrating 14 established pathways regulating cardiac myocyte growth. 109 of 114 model predictions were validated using published experimental data testing the effects of receptor activation on transcription factors and myocyte phenotypic outputs. Network motif analysis revealed an enrichment of bifan and biparallel cross-talk motifs. Sensitivity analysis was used to inform clustering of the network into modules and to identify species with the greatest effects on cell growth. Many species influenced hypertrophy, but only a few nodes had large positive or negative influences. Ras, a network hub, had the greatest effect on cell area and influenced more species than any other protein in the network. We validated this model prediction in cultured cardiac myocytes. With this integrative computational model, we identified the most influential species in the cardiac hypertrophy signaling network and demonstrate how different levels of network organization affect myocyte size, transcription factors, and gene expression.

Finally, while different presentations of hypertrophy are seen in vivo (ex: physiological vs. pathological, eccentric vs. concentric), it is unclear how such a cross-talk dense network could manage these distinct responses. Moreover, little differential regulation is seen among hypertrophy agonists in commonly measured hypertrophy features such as cell size and fetal gene expression. We hypothesized that increasing hypertrophy measurements to include more shape features (ex: elongation and form factor) and expression of other genes relevant in cardiac remodeling (Ex: cell death, fibrosis, proliferation, and inflammation) would allow us to observe more diverse responses among the hypertrophic agonists. 
To test this hypothesis, we stimulated cardiac myocytes with 15 hypertrophic agonists and quantitatively characterized differential regulation of 5 shape features using high-throughput microscopy and transcript levels of 12 genes using qPCR. Transcripts measured were associated with phenotypes including fibrosis, cell death, contractility, proliferation, angiogenesis, inflammation, and the fetal cardiac gene program. While hypertrophy pathways are highly connected, the agonist screen revealed distinct hypertrophy phenotypic signatures for the 15 receptor agonists. We then used kmeans clustering of inputs and outputs to identify a network map linking input modules to output modules. Five modules were identified within inputs and outputs with many maladaptive outputs grouping together in one module: Bax, C/EBP $\beta$, Serca2a, TNF $\alpha$, and CTGF. Subsequently, we identified mechanisms underlying two correlations revealed in the agonist screen: correlation between regulators of fibrosis and cell death signaling (CTGF and Bax mRNA); and myocyte proliferation (CITED4 mRNA) and elongation. Follow-up experiments revealed positive regulation of Bax mRNA level by CTGF and an incoherent feed forward loop linking Nrg1, CITED4 and elongation. With this agonist screen, we identified the most influential inputs in the cardiac hypertrophy signaling network for a variety of features related to pathological and protective hypertrophy signaling and shared regulation among cardiac myocyte phenotypes.

Together, this body of work identified influential network hubs and shared regulation of maladaptive and adaptive hypertrophy features. While this systems approach revealed insights into network organization, it also allowed us to prioritize experiments to reveal new mechanistic insights into hypertrophy, such as the discovery of Ras as the most influential species, CTGF regulation of Bax, and CITED4 regulation of myocyte elongation. The quantitative network understanding gained in this work will be helpful in planning therapeutic interventions for heart failure that enhance adaptive responses and suppresses maladaptive responses. 


\section{List of Figures}

\section{Chapter 1. Background and Significance}

Chapter 2. Automated imaging reveals a concentration dependent delay in reversibility of cardiac myocyte hypertrophy.

2.1 High-throughput image acquisition and analysis platform 20

2.2 Single cross-section of myocyte area is representative of 3D growth 22

2.3 Comparison of data dispersion with and without tracking algorithm 24

2.4 Hypertrophy is reversible at low and persists at high levels of $\alpha$-adrenergic signaling. $\quad 27$

2.5 Residual PE after washout does not explain reversibility delay. 29

2.6 Reversibility of integrated GFP intensity 31

2.7 Increases in fluorescence intensity of cardiac myocytes have minimal effects on cell area $\quad 32$ measurements

2.8 Hypertrophy is reversed by membrane permeable $\alpha A R$-antagonist but not 34 membrane impermeable antagonist

2.9 CGP-12177a is a $\beta 1$-adrenergic receptor antagonist $\quad 35$

2.10 Concentration-dependent reversibility delay may be explained by a ligand trapping model 37

2.11 Model containing only sarcolemmal $\alpha A R^{\prime}$ s cannot reproduce the reversibility delay 38

2.12 Basal growth term is required to reproduce experimental observations 39

2.13 Saturation of degradation of internalized PE is required to fit $1 \mathrm{mmol} / \mathrm{L}$ PE data $\quad 40$

2.14 Saturation of degradation of internalized PE is required to fit across all concentrations $\quad 41$

2.15 Ligand trapping model predicts reversibility of hypertrophy with prazosin 43

2.16 Ligand trapping model predicts longer time course data of $1 \mathrm{mmol} / \mathrm{L} \mathrm{PE}$ treated myocytes 44

2.17 Intracellular $\alpha A R^{\prime}$ s in cardiac myocytes imaged with FL-BODIPY-prazosin 46

Chapter 3. Network reconstruction and systems analysis of cardiac myocyte hypertrophy signaling. 3.1 Schematic of the cardiac hypertrophy signaling network model $\quad 54$

3.2 Hypertrophy signaling network degree distribution $\quad 60$

3.3 Predicting global dynamics of the hypertrophy signaling network 63

$\begin{array}{ll}3.4 \text { Experimental validation of model predictions } & 65\end{array}$

3.5 Partial sensitivity analysis of the hypertrophy signaling network 66

3.6 Comprehensive sensitivity analysis of the hypertrophy signaling network 67

3.7 Correlations between local connectivity, influence, and sensitivity 69

3.8 Schematic of signaling network labeled with degree of influence on cell area 70

3.9 Influence of individual species on cell area $\quad 71$

3.10 Evaluation of model sensitivity to choice of default parameters $\quad 72$

$\begin{array}{ll}3.11 \text { Modular organization of the hypertrophy signaling network } & 74\end{array}$

3.12 Schematic of signaling network with module labeled for each species 75

3.13 Experimental validation of distributed processing by the Ras/MAPK pathway 77

3.14 Fold change in cell area for myocytes treated with inhibitors but without PE $\quad 80$

Chapter 4. Phenotypic screen identifies distinct signatures of cardiac myocyte hypertrophy. 4.1 Phenotypic screen methods and measurements 
4.2 Hypertrophic agonist dose responses for myocyte shape features

4.3 Hypertrophic agonist perimeter dose response data

4.4 Hypertrophic agonist integrated fluorescence intensity dose response data 95

4.5 Intrinsic variability in single-cell data captures distinct modes of shape regulation 97

4.6 Agonist screen identifies distinct hypertrophy phenotypic signatures 99

4.7 Fold change in Bax mRNA compared to control $\quad 100$

4.8 Fold change in Bcl2 mRNA compared to control $\quad 100$

4.9 Fold change in BNP mRNA compared to control 101

4.10 Fold change in C/EBP $\beta$ mRNA compared to control 101

4.11 Fold change in CITED4 mRNA compared to control 102

4.12 Fold change in CTGF mRNA compared to control 102

4.13 Fold change in IKB mRNA compared to control 103

4.14 Fold change in Serca2a mRNA compared to control 103

4.15 Fold change in Skeletal $\alpha$-actin mRNA compared to control 104

4.16 Fold change in TNF $\alpha$ mRNA compared to control 104

4.17 Fold change in VEGF mRNA compared to control 105

4.18 Fold change in myocyte area $\quad 105$

4.19 Fold change in myocyte elongation 106

4.20 Fold change in myocyte form factor 106

4.21 Fold change in myocyte perimeter $\quad 107$

4.22 Fold change in myocyte integrated fluorescence intensity 107

4.23 Clustering reveals modular input-output relationships in hypertrophy signaling 109

4.24 Immunofluorescence imaging of CTGF 111

4.25 Immunofluorescence negative controls for imaging CTGF 112

4.26 CTGF increases Bax mRNA abundance 113

4.27 CITED4 knockdown enhances Nrg1 and LIF-induced myocyte elongation 116

4.28 Computational model lacking CITED4 regulation of myocyte elongation cannot 119

predict enhanced myocyte elongation after CITED4 knockdown

4.29 Computational model lacking minimum myocyte elongation value for CITED4 120

regulation of myocyte elongation cannot predict control CITED4 siRNA data

4.30 Overexpression of CITED4 induces myocyte proliferation and hypertrophy

122

without affecting elongation

Chapter 5. Reverse Phase Protein Array Proteomics Analysis of Hypertrophic Agonists

5.1 Rank ordered list of changes in protein concentration with ET1 treatment 130

5.2 Rank ordered list of changes in protein concentration with Ang II treatment 131

5.3 Rank ordered list of changes in protein concentration with Nrg1 treatment 132

5.4 Rank ordered list of changes in protein concentration with serum treatment 133

5.5 Rank ordered list of changes in protein concentration with IGF1 treatment 134

5.6 Hierarchical clustering of the RPPA data at 1 hour and 48 hours 136

5.7 Hierarchical clustering of the combined RPPA data at both time points 137

\section{Chapter 6. Dissertation conclusions and future directions}

6.1 Predicted and measured changes in cell area in response to increasing concentrations of

hypertrophic agonists

6.2 Model prediction of hypertrophy signaling network dose response for each receptor

146 agonist 


\section{List of Abbreviations}

aAR $\quad \alpha$-adregergic receptor

Ang II Angiotensin II

ANP Atrial naturetic peptide

BNP Brain naturetic peptide

CITED4 Cbp/P300 interacting transactivator with Glu/Asp-rich carboxy-terminal domain, 4

CT1 Cardiotrophin 1

CTGF Connective tissue growth factor

EGF Epidermal growth factor

ET1 Endothelin 1

FGF2 Fibroblast growth factor 2

IL6 Interleukin-6

Iso Isoproterenol

LIF Leukemia inhibitory factor

NE Norepinephrine

Nrg1 Neuregulin 1

PE Phenylephrine

RPPA Reverse Phase Protein Array

SACT skeletal $\alpha$-actin

SERCA Sarcoplasmic reticulum ATPase

TGF $\beta$ Transforming growth factor $\beta$

TNF $\alpha$ Tumor necrosis factor $\alpha$ 
Chapter 1

\section{Background and Significance}




\subsection{Cardiac hypertrophy is a leading predictor of heart failure}

Cardiovascular disease is the most common cause of death and disability in industrialized countries and heart failure is currently considered to be the biggest challenge in cardiovascular medicine[1]. In 2012, 2.4\% of the United States population had heart failure[2], which is characterized by a decrease in the ability of the heart to adequately pump blood in response to the body's demands[3]. Despite significant therapeutic improvements over the last half century, the prognosis for heart failure patients remains bleak[4]. Symptomatic heart failure has a five-year mortality rate of more than 50 percent[5], [6], a prognosis worse than most cancers[7-9]. Cardiac hypertrophy, the increase in heart size due to enlargement of cardiac myocytes, is a leading predictor for heart failure[10], [11].

Hypertrophy develops as a result of increases in biochemical and mechanical stresses on the heart. Stresses from exercise or pregnancy induce physiological growth of the heart and stresses from hypertension, myocardial injury, and neurohumoral activation can lead to pathological growth[12]. The growth of individual cardiac myocytes is triggered by mechanical stimuli and stress-released hormones, cytokines, chemokines, and growth factors that lead to activation of hypertrophic signaling pathways and changes in gene expression[13]. Data from humans subjects confirmed that left ventricle hypertrophy is an independent risk factor for heart failure and that decreasing left ventricle mass reduces mortality[14]. Therefore prevention and reversal of hypertrophy are key objectives for advancing therapies for heart failure.

Despite the need for therapeutic advances, little drug development activity by pharmaceutical companies is ongoing for this syndrome, partially due to the high costs of running large cardiovascular outcome studies, with costs to develop a new agent potentially exceeding a billion dollars[15]. The large unmet medical need in heart failure demands the use of new approaches for developing therapies for heart failure that will improve the probability of success of clinical trials[16]. 


\subsection{Unique signaling in pathological and physiological hypertrophy}

Pathological hypertrophy is characterized with increased fibrosis, cell death, and cardiac dysfunction while physiological hypertrophy is reversible and characterized with normal or improved cardiac function without cell death and fibrosis[17]. While the mechanisms that distinguish pathological and physiological hypertrophy are unclear[18], differences in gene expression[19] and signaling pathway activation between these two forms of hypertrophy have been observed. Insulin-like growth factor 1 (IGF1) levels in the heart were upregulated in swim-trained rats[20] and professional athletes[21]. Conversely, pressure overload increased levels of Angiotensin II, catecholamines, and endothelin-1[2224]. Mice with reduced cardiac PI3K activity showed a reduced hypertrophic response to swimming, but not pressure overload induced hypertrophy[25], while rats with Angiotensin II receptor inhibition showed reduced pressure overload hypertrophy[26], but not exercise induced hypertrophy[27]. Increased understanding about the specific pathways controlling physiological and pathological hypertrophy is needed to develop advanced therapies for heart failure that enable physiological, adaptive growth and prevent pathological growth[17], [18], [28].

\subsection{Unique growth responses to different stress types}

In addition to physiological and pathological hypertrophy, myocytes grow distinctly in response to different stimuli. Pressure overload due to stimuli such as hypertension, aortic stenosis, and strength training leads to increases in systolic wall stress and concentric hypertrophy, where the cardiac wall thickens. Conversely, volume overload due to stimuli such as aortic regurgitation, arteriovenous fistulas, aerobic exercise, and pregnancy increases diastolic wall stress and results in eccentric hypertrophy, where the left ventricle dilates[29]. Little is known about the specific signaling pathways that determine myocyte elongation and eccentric hypertrophy versus increased myocyte thickness and concentric hypertrophy[30]. Since eccentric hypertrophy is a greater risk to patients than concentric 
hypertrophy[31] , increased knowledge of the unique signaling pathways controlling these growth patterns will be vital in improving therapies for heart failure. 


\subsection{Network complexity motivates use of systems methods}

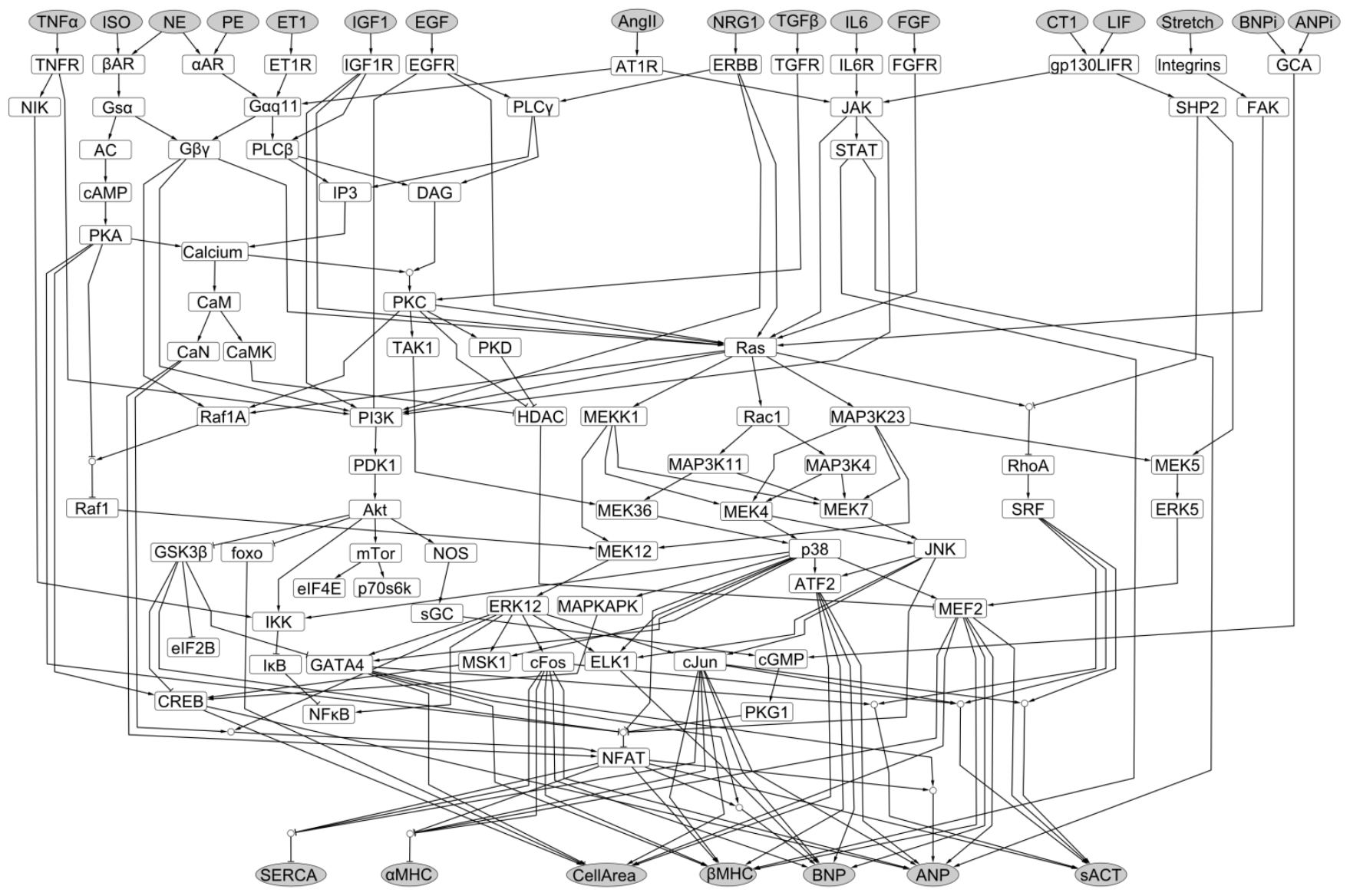

Figure 1.1. Diagram of major pathways in the cardiac myocyte hypertrophy signaling network. 
The cardiac hypertrophy response is controlled by a dense web of signaling pathways, with many molecular species influencing cardiac myocyte growth [3] (Figure 1.1). While many of the components of this network have been identified, the distinct contributions to different features of hypertrophy such as elongation, fibrosis, and cell death between signaling pathways is not understood[32]. This information will be essential in determining how these biological circuits can be manipulated therapeutically to prevent and reverse heart failure. The complexity of the cardiac hypertrophy signaling network indicates that integrative systems approaches, which can provide a global view of functional relationships in the network, will be essential in accomplishing these goals[33].

Current approaches to studying hypertrophy have been low-throughput and qualitative, probing isolated pathways. These studies are useful in determining a functional role of a gene or protein, but experiments providing quantitative, temporal, and spatial information are needed to fully understand network organization in a complex process like cardiac hypertrophy [34]. This will require new large scale experimental approaches that can measure a large number of input and output relationships at multiple time points [13]. One promising approach is high-content cellular imaging, which can quickly and reproducibly generate high volumes of quantitative data across spatial and temporal dimensions[35]. Here, we developed a new high-throughput experimental approach needed for systems analysis of the cardiac myocyte hypertrophy signaling network. We used this platform to better characterize the phenotypic outputs of predominant hypertrophic pathways and test hypotheses about molecular species that regulate distinct forms of hypertrophy.

\subsection{Significance}

Previous work on hypertrophy has primarily only examined individual pathways in a given study, often with little quantification. In this work we take a more integrative approach to studying hypertrophy by comparing the effects of many pieces of the network in a single study, providing an 
opportunity to analyze the relative contribution of pathways to various features associated with cardiac hypertrophy as well as the influence of interactions between pathways.

Since hypertrophy is controlled by a dense signaling network, this global view will be essential for developing innovative therapies[34]. This systems approach will reveal common network modules and pathways that control specific aspects of hypertrophy[36]. This knowledge will increase understanding on the molecular basis for physiological versus pathological hypertrophy and eccentric versus concentric hypertrophy, which will be critical for engineering more specifically targeted therapies for cardiac hypertrophy.

Current methods to studying hypertrophy have heavily relied on low-throughput methods such as manual segmentation of immunofluorescence images of cardiac myocytes or in vivo rodent models. These methods have been useful in providing functional information about network species, but provide limited quantitative shape data and accommodate limited numbers of perturbations in a single study. Current automated myocyte image segmentation methods for hypertrophy have used fixed cells[37], [38], which prevent scalable studies of the kinetics or reversal of hypertrophy. Our new live cell automated imaging and analysis platform allows us to quickly and reproducibly track and analyze thousands of cardiac myocytes with minimal user input, making feasible the large number of perturbations needed for systems studies of cardiac myocyte hypertrophy signaling[39].

\subsection{Specific Aims}

For this dissertation, our overall objective was to integrate experimental and computational approaches to determine how the components and network topology of hypertrophy signaling lead to differential regulation of myocyte shape and gene expression with the following specific aims:

Aim 1: Develop an automated microscopy and image processing platform for cardiac myocyte hypertrophy and validate this platform by studying the reversibility kinetics of phenylephrine-induced hypertrophy. To understand network organization in a complex process like cardiac hypertrophy, new 
large scale experimental approaches are needed that can measure a large number of input-output relationships at multiple time points. We developed a platform that tracked changes in shape of thousands of individual cardiac myocytes over time, enabling the study of kinetics and reversibility of hypertrophy. We validated this imaging platform by studying the dynamics of reversal of phenylephrineinduced hypertrophy at multiple concentrations.

\section{Aim 2: Develop a large-scale computational model of the hypertrophy signaling network.} Computational models have been used to increase understanding of the role of signaling components and topology on cardiac physiology such as myocyte contractility, arrhythmia, and hypertrophy. Although these models have been used successfully to address focused questions about individual pathways, more global network reconstructions are needed to understand differential regulation of hypertrophy and crosstalk between pathways. We developed a computational model of hypertrophy by integrating the most established pathways implicated in cardiac myocyte growth and using the normalized Hill equation modeling approach with default parameters. Sensitivity analysis informed clustering of the network into modules and identified species with the greatest effects on cell growth.

\section{Aim 3: Quantitatively characterize differential regulation of hypertrophy phenotypes induced} by 15 predominant hypertrophic agonists. We developed a computational model with 106 signaling effectors, combining 14 established pathways involved in cardiac myocyte hypertrophy. Model analysis revealed that while many of these established pathways predict the increases in cell area induced by each agonist, these pathways cannot explain the differential regulation of myocyte shape and gene expression induced by each agonist. Our new automated microscopy and image analysis platform enables quantitative comparisons of changes in area, elongation, form factor, perimeter, and integrated fluorescence intensity between pathways. We performed qPCR for 12 genes associated with fibrosis, apoptosis, contractility, proliferation, and inflammation, providing a hypertrophy phenotypic signature 
for each ligand. Differential expression of these genes helped associate measured changes with physiological or pathological features of hypertrophy. We used statistical analysis tools to find new modules and links between inputs and outputs and validate mechanisms underlying a subset of these relationships using our automated imaging platform.

These aims elucidate mechanisms that control how the densely connected hypertrophy signaling network manages a variety of different hypertrophy phenotypic outputs. This quantitative network understanding is needed to understand and ultimately control the differential regulation of myocyte shape and gene expression in cardiac myocyte hypertrophy. 


\section{Chapter 2}

\section{Automated imaging reveals a concentration dependent delay in reversibility of cardiac myocyte hypertrophy.}

Reprinted from: Automated imaging reveals a concentration dependent delay in reversibility of cardiac myocyte hypertrophy, Vol. 53, Issue 2, August 2012, Pages 282-90, with permission from Elsevier. 


\subsection{Introduction}

Cardiac hypertrophy develops in response to stresses on the heart as the body attempts to compensate for reduced cardiac output. While this response may be adaptive initially, if stresses persist, an apparently irreversible decompensation can occur leading to heart failure [12]. A dense signaling network manages this response, with numerous pathways implicated in the hypertrophy phenotype [3]. A quantitative understanding of the pathways and their interactions is needed to understand how context dependent decisions are made by myocytes to control heart growth and in order to develop more effective therapies for heart failure.

Reversing hypertrophic remodeling remains a key objective for advancing therapies for heart failure. Nearly every successful clinical treatment for heart failure associated with improvements in longterm clinical outcomes reverses remodeling [14]. Despite this, little is known about the specific signaling pathways that distinguish reversible forms of hypertrophy from irreversible forms which lead to heart failure. Few comprehensive studies of hypertrophy reversibility have been conducted and none in a cell culture model. Previous studies have shown reverse remodeling in mouse models [40-42] and in patients after medical device implantation or other surgical interventions [43-45]. While these studies support the idea that targeting reversibility of pathological hypertrophy may be a viable therapeutic strategy, studying reversibility of hypertrophy in a cell model will be essential for systematically exploring reversibility mechanisms and screening for potential drug targets.

Current approaches to study hypertrophic signaling have been low-throughput and qualitative, probing isolated pathways. These studies are useful in determining a functional role of a gene or protein, but experiments providing quantitative, temporal, and spatial information are needed to fully understand network organization in a complex process like cardiac hypertrophy [34]. This will require new large scale experimental approaches that can measure a large number of input and output relationships at multiple time points [13]. One promising approach is high-content cell imaging, which 
can quickly and reproducibly generate high volumes of quantitative data across spatial and temporal dimensions [35]. Recently automated segmentation has been used to study cardiac hypertrophy, but only in fixed cells [37], [46].

Here, we developed a high-throughput screening procedure to study the hypertrophy signaling network in live cardiac myocytes. Using automated large-scale microscopy and image analysis, thousands of individual cardiac myocytes were measured and tracked over several days. This new platform was applied to study reversibility kinetics of phenylephrine (PE)-induced cardiac myocyte hypertrophy. Our approach revealed that PE-induced hypertrophy exhibits a concentration-dependent reversibility delay, with sustained hypertrophy after agonist washout in myocytes exposed to high initial concentrations of PE. Additional perturbations and a mathematical model demonstrate that this reversibility delay can be mechanistically explained by internalized PE acting on intracellular $\alpha$ adrenergic receptors.

\subsection{Materials and methods}

\subsubsection{Cell culture}

Cardiac myocytes were harvested from 1-2 day old Sprague Dawley rats using the Neomyts isolation kit (Cellutron, Baltimore, MD). All procedures were performed in accordance with the Guide for the Care and Use of Laboratory Animals published by the US National Institutes of Health and approved by the University of Virginia Institutional Animal Care and Use Committee. Myocytes were cultured in plating media (Dulbecco Modified Eagle Media, 17\% M199, 10\% Horse Serum, 5\% Fetal Bovine Serum, $100 \mathrm{U} / \mathrm{mL}$ penicillin, and $50 \mathrm{mg} / \mathrm{mL}$ streptomycin) on Cellbind treated 96 -well plates (Corning, Corning, NY) at a density of 100,000 cells/well. Two days after isolation, myocytes were transfected with GFP driven under a cardiac myocyte specific troponin T promoter [47] using Lipofectamine 2000 (Invitrogen, Carlsbad, California; transfection efficiency: 10-15\%). 
Two days after transfection, myocytes were imaged using an Olympus IX81 inverted microscope with 10X UPlanFLN 0.30 NA objective, Orca-AG CCD camera (Hamamatsu, Bridgewater, NJ), automated stage (Prior Scientific, Rockland, MA), and IPLab software (Scanalytics, Fairfax, VA). Images were acquired with 120 ms exposure time using a 480/40-nm excitation filter and 535/50-nm emission filter (Chroma filters; Optical Insights, Santa Fe, NM). After imaging, cells were rinsed and transferred to serum-free media (Dulbecco Modified Eagle Media, 19\% M199, 1\% ITSS, 100U/mL penicillin, and 50 $\mathrm{mg} / \mathrm{mL}$ streptomycin) with a given concentration of $\mathrm{PE}$, an $\alpha$-adrenergic receptor ( $\alpha \mathrm{AR}$ ) agonist. After 24 hours, cells were imaged, rinsed twice, and cultured in serum-free media without PE. Follow-up images were recorded every 24 hours.

\subsubsection{Automated microscopy}

Software algorithms were developed to automatically focus, capture a $5 \times 5$ grid of images in each well of interest in a 96-well plate, and assemble these images into a composite mosaic image. This allows for a highly reproducible imaging protocol in which the same region of cells can be imaged and tracked over the entire four day imaging period. Mosaic images enhance the number of cells imaged per well. The $5 \times 5$ mosaic encompasses an approximately $0.06 \mathrm{~cm}^{2}$ region, which is about $20 \%$ of the cell growth area of a well and contains 150 GFP-expressing cardiac myocytes. In contrast, a single image captures less than $1 \%$ of the cell growth area of a well and on average contains less than 10 myocytes.

Using centroid coordinates for a 96-well plate, the imaging software directed the motion of the stage to the center of each well in a left to right serpentine motion (Figure 2.1A). Within each well, an autofocus operation was performed by positioning the objective to five different heights, $50 \mu \mathrm{m}$ apart, and recording an image. The z-step resulting in the highest contrast image was selected and then the process was repeated at five smaller z-step intervals $2 \mu \mathrm{m}$ apart. The objective height resulting in the highest contrast image of the $2 \mu \mathrm{m}$ z-steps was the focus used for image collection in that well. 


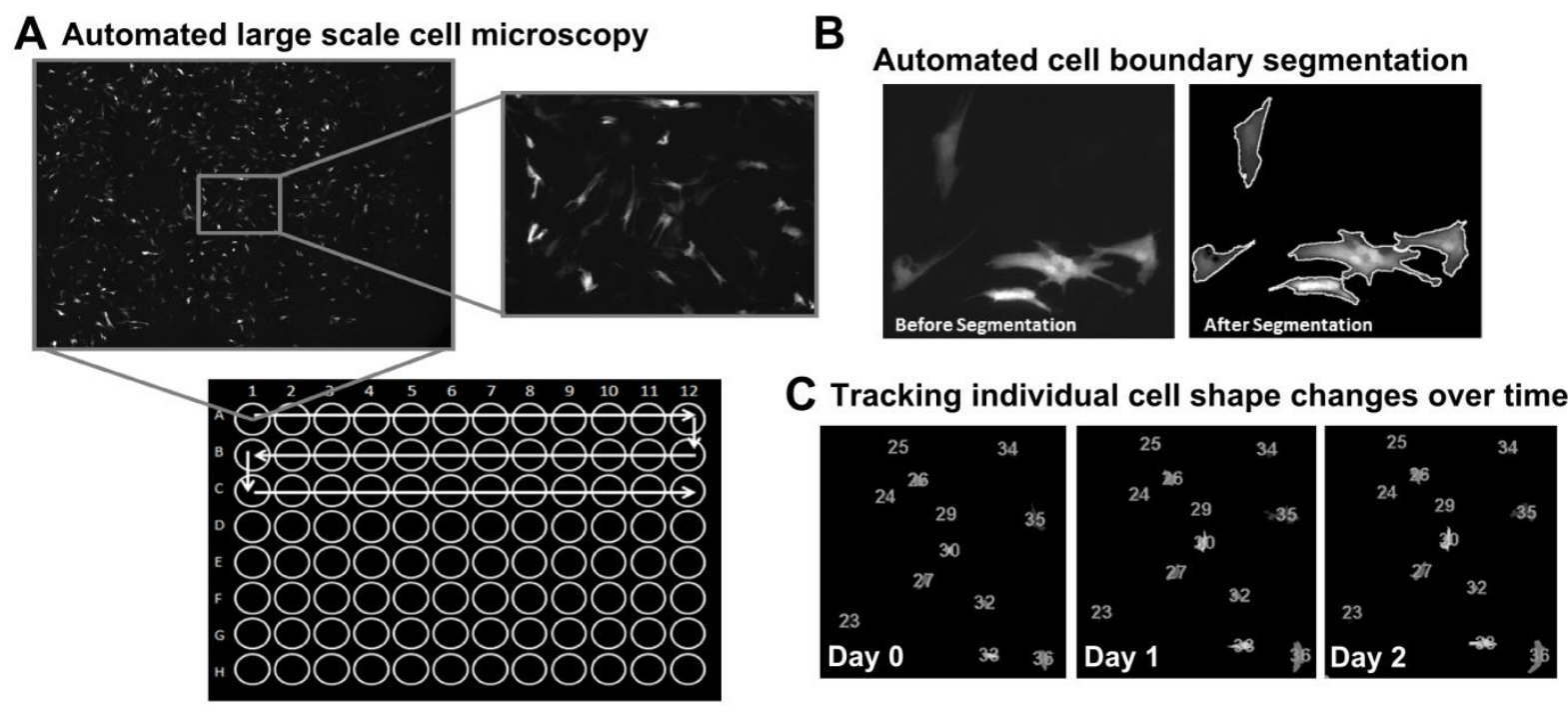

Figure 2.1: High-throughput image acquisition and analysis platform can track morphological dynamics of individual cardiac myocytes. A) Scripts to control the microscope's automated stage allowed for highly reproducible imaging of $5 \times 5$ mosaics images of GFP-labeled cardiac myocytes within each well of a 96-well plate. These mosaic images maximize the cell data collected per well/perturbation, including approximately 100-200 cells per well. B) Example output cell boundary segmentations from automated image analysis algorithm. Using these segmentations, a variety of cardiac myocyte morphology measurements can be calculated. C) Example output from cardiac myocyte tracking over 3 days. Cells are labeled and tracked between measurements based on the proximity to the cell's location on the preceding day. 
The automated stage then moved to each position in a $5 \times 5$ grid about the centroid of the well in a left to right serpentine motion, capturing an image at each location. This grid of images was then assembled into a composite mosaic image (Figure 2.1A) and saved with a filename labeling the coordinates of the well on the 96-well plate. This process was repeated for every well of interest on the plate. Imaging for these experiments lasted 10-20 minutes and cells were kept in an incubator between imaging periods.

\subsubsection{Automated image analysis}

Based on the filename, the algorithm first loads the four $5 \times 5$ mosaic images saved in 16-bit Tiff format of the same well imaged on four different days. A threshold of 0.01 is set to reduce background noise and the intensities are rescaled to a range from 0 to 1 , which is a requirement for the segmentation step in the algorithm. Next, a pixel intensity that distinguishes objects from background is found using the Otsu method [48].

An advantage of this approach is that given the transfection efficiency $(10-15 \%)$ using Lipofectamine, expressing cells are rarely adjacent to each other, allowing for conspicuous cell boundaries. However, in the event of a cluster of expressing myocytes, cells are differentiated by intensity value, since GFP expression tends to be higher in the center of the cell. The number of cells in a group is determined by counting the number of local maxima of intensity in a smoothed image. Then dividing lines between adjacent cells are calculated with a watershed algorithm [49] using the previously identified local maxima of intensity as starting points. Any objects touching the border of the image are discarded.

Using the identified cell boundaries (Figure 2.1B), various metrics of cell shape are calculated for each cell including area, perimeter, eccentricity, major axis length, minor axis length, form factor, and orientation. Here, the output of interest was cell area. We validated the use of myocyte area as 


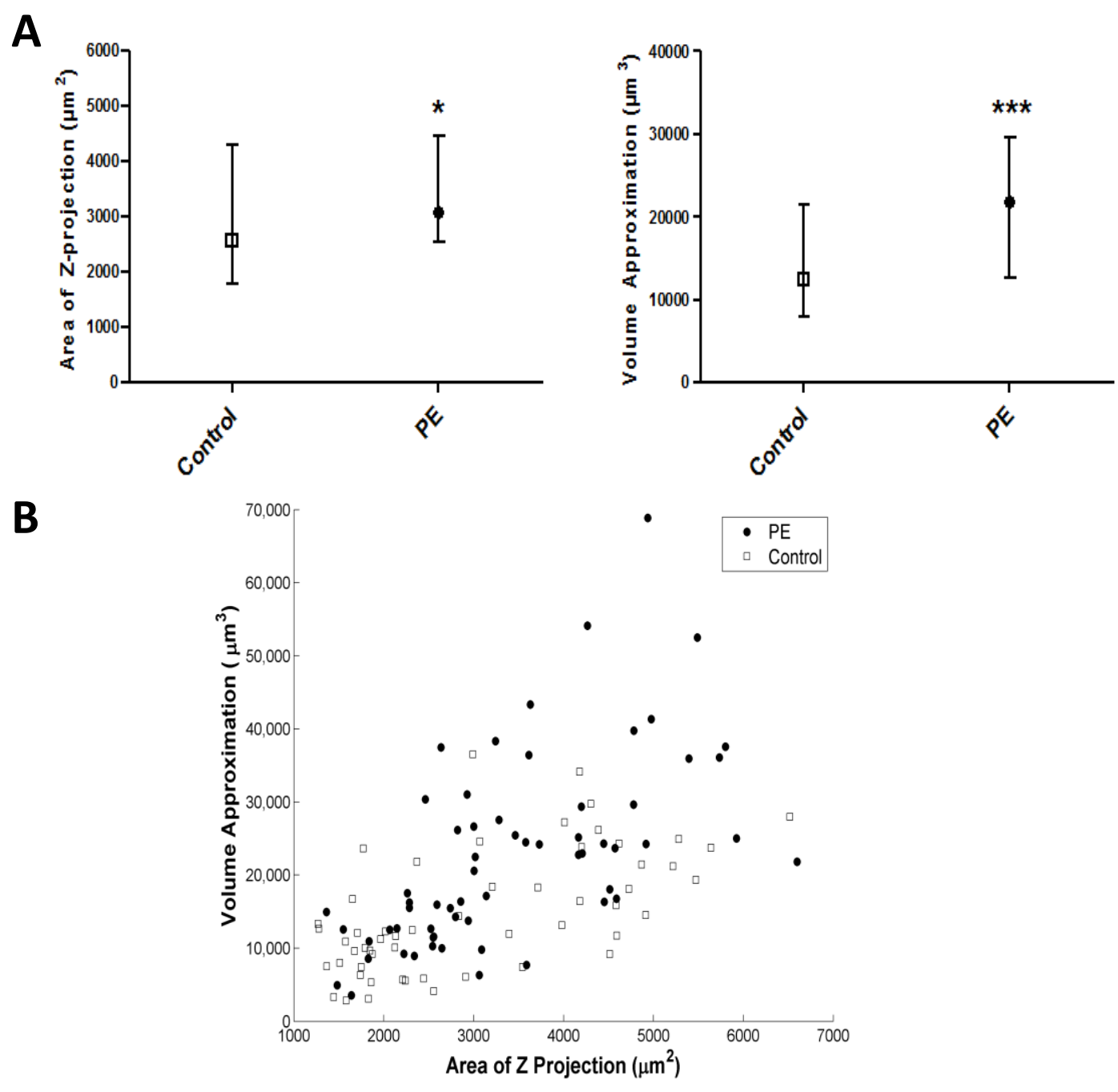

Figure 2.2: Single cross-section of myocyte area is representative of 3D growth for PE-induced hypertrophy. 3D image stacks taken at 1- $\mu \mathrm{m}$ z-steps were collected of cardiac myocytes transfected with GFP driven under a cardiac myocyte specific troponin T promoter. Images were collected with a Zeiss LSM 510 META laser scanning microscope with a 25X water Plan-Neofluar 0.8 NA objective, LSM 4.0 META software, and a $40 \mathrm{~mW}$ Argon-ion laser generating the 488-nm line. Images were median filtered with 2.0 radius window to reduce background and then image volumes were estimated by summing myocyte cross-sectional areas at 1- $\mu \mathrm{m}$ steps throughout the entire cell using the 3D object counter plugin for ImageJ. The summation of the image stack was calculated to get the Z-projection, which approximates the signals that would be recorded using widefield epifluorescence microscopy, which collects light throughout every z-plane of the specimen. Cell areas of the Z-projection were calculated using our automated image analysis pipeline described in the main text. a) Myocytes cultured in a solution of $10 \mu \mathrm{mol} / \mathrm{LPE}$ in serum free media for 24 hours were compared to myocytes kept in serum free media (control). Plots display the median and interquartile range ( 60 cells per condition) of Z-projection area (left) and volume approximation (right) for both conditions. Myocytes with PE had significantly larger Z-projection area $\left({ }^{*} P<.05\right)$ and cell volume $\left({ }^{* * *} P<.001\right)$. Differences in area and 
volume were tested for statistical significance using a Mann-Whitney test. Results indicate that PE induces myocyte growth in all dimensions and that recorded increases in cell area are not exclusively increases in cell attachments and spreading. b) Scatter plot of $Z$ projection area and volume data for cardiac myocytes in both conditions. To validate our use of cross-sectional area as a measurement of cardiac myocyte hypertrophy we calculated the correlation of area of the Z-projection with our approximation of cell volume. The Pearson's correlation coefficient, which included data from both conditions, was 0.67 , indicating a strong correlation between these two measures and validating our use of cross-sectional area as representative of three-dimensional cardiac myocyte growth for PE-induced cardiac myocyte hypertrophy. 

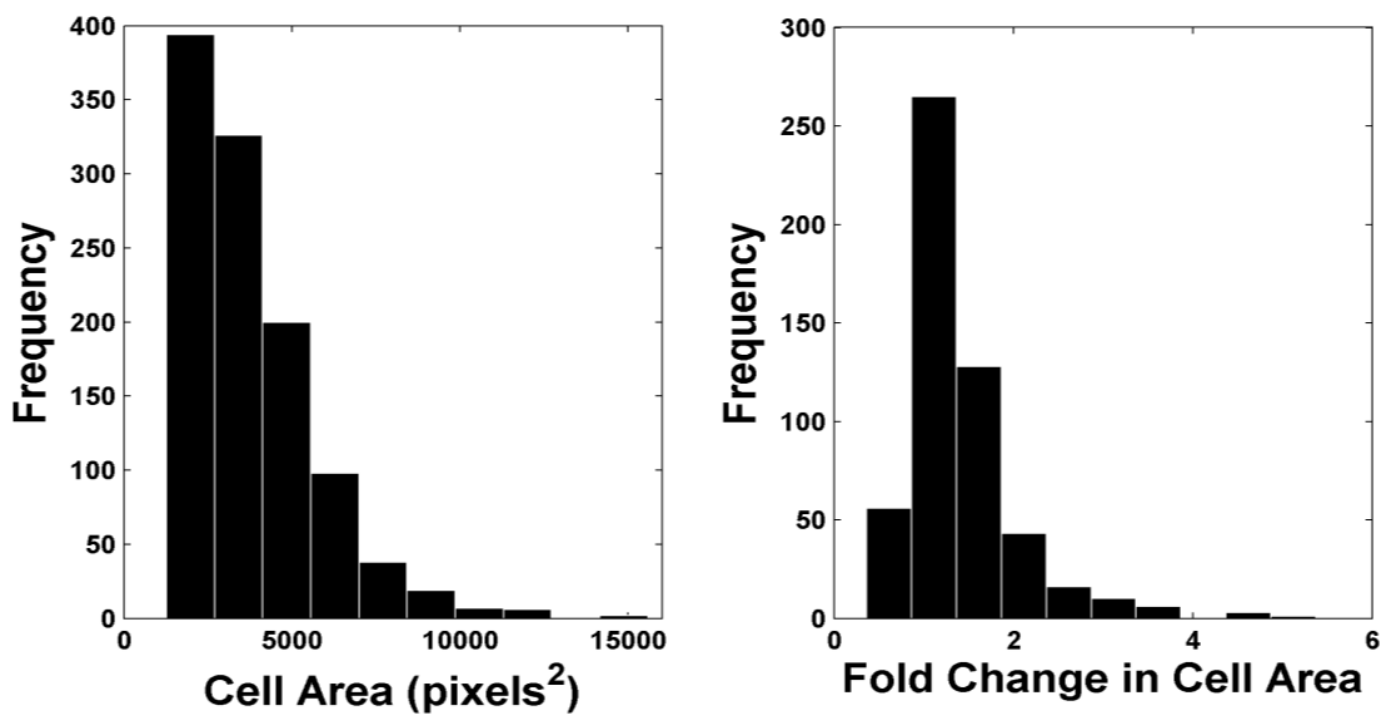

Figure 2.3: Comparison of data dispersion with and without tracking algorithm. Fold change data output from the tracking algorithm have less dispersion and a more peaked histogram than the raw area data collected without tracking. This provides evidence of the increased precision for calculating changes in area attained using our data analysis approach with myocyte tracking. Example data shown are from the $100 \mu \mathrm{mol} / \mathrm{L}$ PE condition: day 1 cell areas and fold changes in cell area between day 1 and 2. Moreover, normalized $90 \%$ confidence intervals of the median calculated through bootstrapping were an average of $77 \%$ larger for cell measurements without tracking. Bootstrapped estimates were calculated by first taking 50 point samplings of the dataset with replacement and calculating the sample median for $\mathrm{N}=200$ different samplings. Then the .05 and .95 quantiles were calculated for these 200 estimates of the median. This process was repeated 10 times and averaged to give a final estimate of the $90 \%$ confidence intervals. For example, the day $1100 \mu \mathrm{mol} / \mathrm{L}$ data had $90 \%$ confidence intervals of 1.16-1.37 for normalized fold change in area with tracking and 2827-3779 for area in square pixels without tracking. This generated a normalized range in $90 \%$ confidence interval of 0.17 and 0.29 respectively, with $70 \%$ wider normalized confidence intervals without cell tracking. In addition, the normalized interquartile range was $65 \%$ larger without tracking. The reduction in data dispersion with tracking provides evidence for the method's increased precision in the estimate of the median and increased statistical power. Therefore, tracking individual cells increases the ability to resolve changes occurring to a cell population with inherent biological variability after exposure to a hypertrophic agonist. 
representative of three-dimensional cell growth for PE-induced cardiac myocyte hypertrophy (Figure 2.2). After segmenting the first image of the four day sequence, each cell is given an ID number and tracked between subsequent images based on distance - the closest cell in the next image to its original position is labeled with the same ID number. The tracking algorithm searches a neighborhood of 100 pixels surrounding the previous location of each identified myocyte. Since cardiac myocytes migrate minimally, the myocytes stayed in the same location between subsequent images and distance was a robust method of tracking the myocytes (Figure 2.1C). The ID number of each cell is saved in the output file, so that the measurements can be sorted in post-processing. The tracking step in this algorithm therefore allows for changes in measurements of individual myocytes to be observed over time. Fold change data output using the tracking algorithm have less dispersion and a more peaked histogram than the raw area data collected without tracking (Figure 2.3).

The automated cell segmentation and tracking algorithm was implemented using the opensource MATLAB-based CellProfiler software package [50]. Matlab scripts were developed to sort area measurements based on the saved cell ID numbers. Only myocytes with an area measurement for all four days were included in our data analysis. Detailed methods for developing automated image acquisition and analysis pipelines are available in Appendix A.

\subsubsection{BODIPY-prazosin competitive inhibition experiments}

Cardiac myocytes were given a $10 \mathrm{nmol} / \mathrm{L}$ solution of BODIPY-FL-prazosin (Molecular Probes) in serum free media for five minutes and then imaged using a Zeiss LSM 510 META laser scanning microscope with a 63X oil Plan Apochromat 1.4 NA objective, LSM 4.0 META software, and a $40 \mathrm{~mW}$ Argon-ion laser generating the 488-nm line. 3D stacks were taken for a subset of the imaged myocytes at $0.5 \mu \mathrm{m}$ z-steps. For competitive inhibition experiments, myocytes were preincubated with a solution of $10 \mu \mathrm{mol} / \mathrm{L}$ phentolamine, $1 \mathrm{mmol} / \mathrm{L} \mathrm{PE}$, or $100 \mu \mathrm{mol} / \mathrm{L}$ CGP-12177a in serum free media for ten minutes before adding BODIPY-prazosin, giving a final concentration of $10 \mathrm{nmol} / \mathrm{L}$ BODIPY-prazosin. 
Images were collected after myocytes were in solution with BODIPY-prazosin for five minutes. BODIPYprazosin labeling was quantified by segmenting the imaged myocytes using the phase-contrast channel and then calculating the above threshold integrated intensity of the BODIPY-prazosin channel normalized by cell area for each myocyte imaged. The threshold was set as the pixel intensity three times above the mean background intensity in the BODIPY-prazosin channel. The median and interquartile range were calculated for each condition. Differences in above threshold integrated intensity/cell area were tested for statistical significance using Kruskall-Wallis non-parametric one-way analysis of variance followed by a Dunn's multiple comparisons post-test.

\subsection{Results}

\subsubsection{Concentration dependent reversibility of PE-induced hypertrophy}

This automated imaging platform provides a unique opportunity to examine the reversibility of cardiac myocyte hypertrophy. Previous cellular studies have primarily examined single time points, preventing the observation of dynamics of cell growth generated by various hypertrophic agonists. Since this platform can track morphological changes in individual cardiac myocytes, mechanistic studies of hypertrophy reversibility are possible. Here, we studied the reversibility of cell growth after application of several different concentrations of PE.

After recording initial images (day 0), the cardiac myocytes were cultured in serum-free media containing varying PE concentration for 24 hours and then rinsed and switched to serum-free media without PE. Images were collected every 24 hours. On day 1, myocytes exhibited concentrationdependent increases in cell area, as expected, highlighting this platform's ability to quantify distinct levels of cell growth (Figure 2.4A). Levels of PE-induced hypertrophy are consistent with the range reported in the literature using other imaging methods [37], [51], [52]. After PE washout, myocytes exposed to lower concentrations ( $\mathrm{PE} \leq 10 \mu \mathrm{mol} / \mathrm{L}$ ) began decreasing in size, reverting to approximately their original 
A

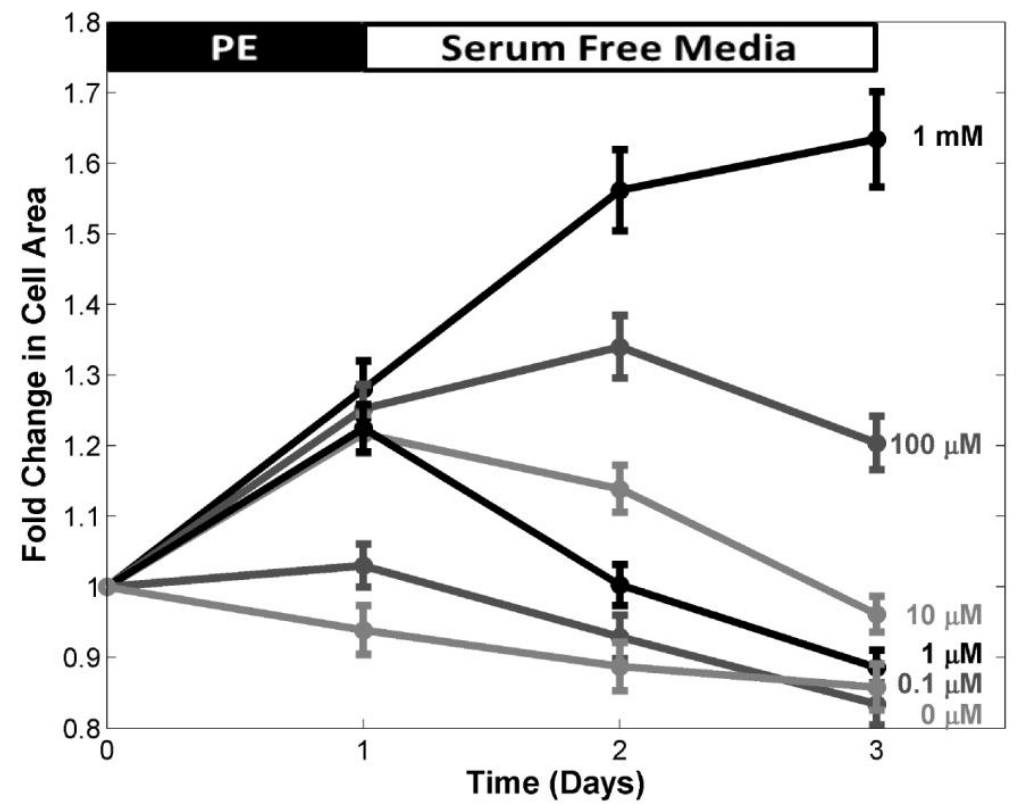

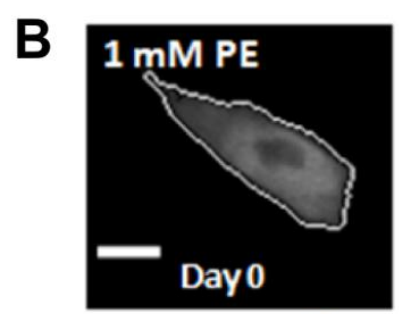

$316 \mu \mathrm{m}^{2}$

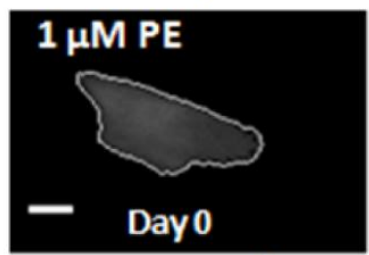

$516 \mu \mathrm{m}^{2}$

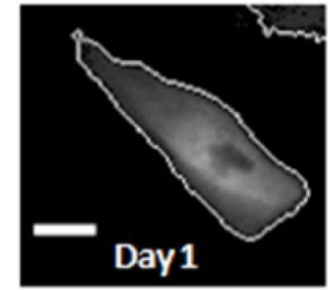

$426 \mu \mathrm{m}^{2}$

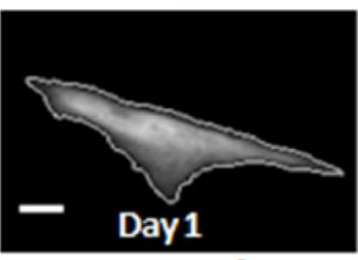

$603 \mu \mathrm{m}^{2}$

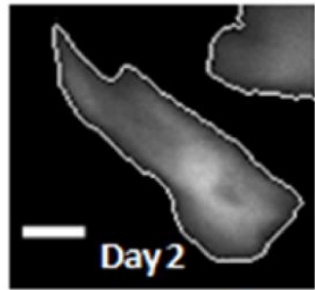

$528 \mu \mathrm{m}^{2}$

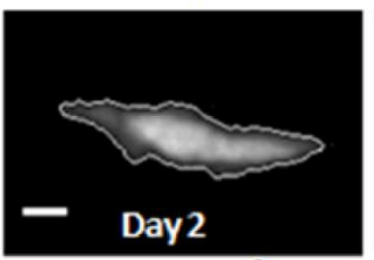

$516 \mu \mathrm{m}^{2}$

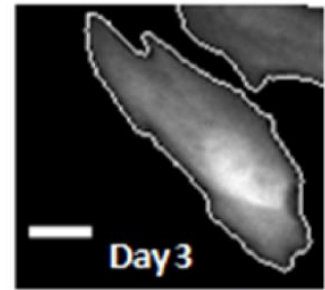

$581 \mu \mathrm{m}^{2}$

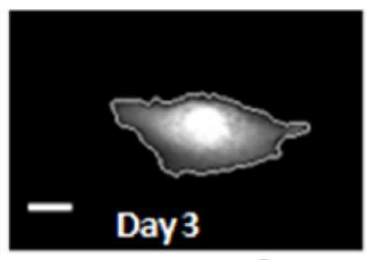

$501 \mu \mathrm{m}^{2}$

Figure 2.4: Hypertrophy is reversible at low and persists at high levels of $\alpha$-adrenergic signaling. A) Time course data of median ( 400 cells per condition) fold change in cell area of cardiac myocytes after 24 hours exposure to a given concentration of PE in serum free media. On day 1 the agonist was washed out and replaced with serum free media. Error bars are +/- SE. B) Representative images with labeled cell areas (Scale bar: $10 \mu \mathrm{m}$ ) of segmented cardiac myocytes exposed to $1 \mathrm{mmol} / \mathrm{L} \mathrm{PE} \mathrm{(top)} \mathrm{and} 1 \mu \mathrm{mol} / \mathrm{L}$ $P E$ (bottom). Cells exposed to $\geq 100 \mu \mathrm{mol} / \mathrm{L}$ PE continue to increase in size after the agonist is washed out of the extracellular media. 
area within 48 hours after washout. Conversely, myocytes exposed to the highest concentrations

$(\mathrm{PE} \geq 100 \mu \mathrm{mol} / \mathrm{L})$, continued to increase in area after agonist washout, with the $1 \mathrm{mmol} / \mathrm{L}$ condition showing increasing fold changes in cell area throughout the entire 72-hour data collection period. Figure 2.4B shows representative cells exposed to $1 \mu \mathrm{mol} / \mathrm{L}$ or $1 \mathrm{mmol} / \mathrm{L} \mathrm{PE}$.

Differences in fold change in cell area on day 1 and day 3 were tested for statistical significance using Kruskall-Wallis non-parametric one-way analysis of variance followed by a Dunn's multiple comparisons post-test test (Table 2.1). To confirm that this finding was not the result of residual PE after washout in the high concentration conditions, we also measured cell growth in myocytes exposed to PE for 30-second intervals (Figure 2.5).

\section{PE-induced hypertrophy reversibility}

\begin{tabular}{|l|l|l|}
\hline Dunn's Multiple Comparison Test & Significant, $\boldsymbol{P}<\mathbf{0 . 0 5}$ \\
\hline Fold Change in Cell Area on & Day 1 & Day 3 \\
\hline $\mathbf{0} \mu \mathrm{M}$ PE vs $0.1 \mu \mathrm{M}$ PE & Yes & No \\
\hline $\mathbf{0} \mu \mathrm{M}$ PE vs $1 \mu \mathrm{M}$ PE & Yes & No \\
\hline $0 \mu \mathrm{M}$ PE vs $10 \mu \mathrm{M}$ PE & Yes & Yes \\
\hline $0 \mu \mathrm{M}$ PE vs $100 \mu \mathrm{M}$ PE & Yes & Yes \\
\hline $\mathbf{0} \mu \mathrm{M}$ PE vs $1 \mathrm{mM}$ PE & Yes & Yes \\
\hline
\end{tabular}

Table 2.1: Differences in fold change in cell area on day 1 and day 3 in data from PE-induced hypertrophy reversibility experiments were tested for statistical difference from control ( $0 \mu \mathrm{mol} / \mathrm{L} P E)$ using Kruskall-Wallis non-parametric one-way analysis of variance followed by a Dunn's multiple comparisons post-test test. $\mathrm{N} \sim 400$ cells per condition, $\mathrm{P}<0.05$ was considered significant. 


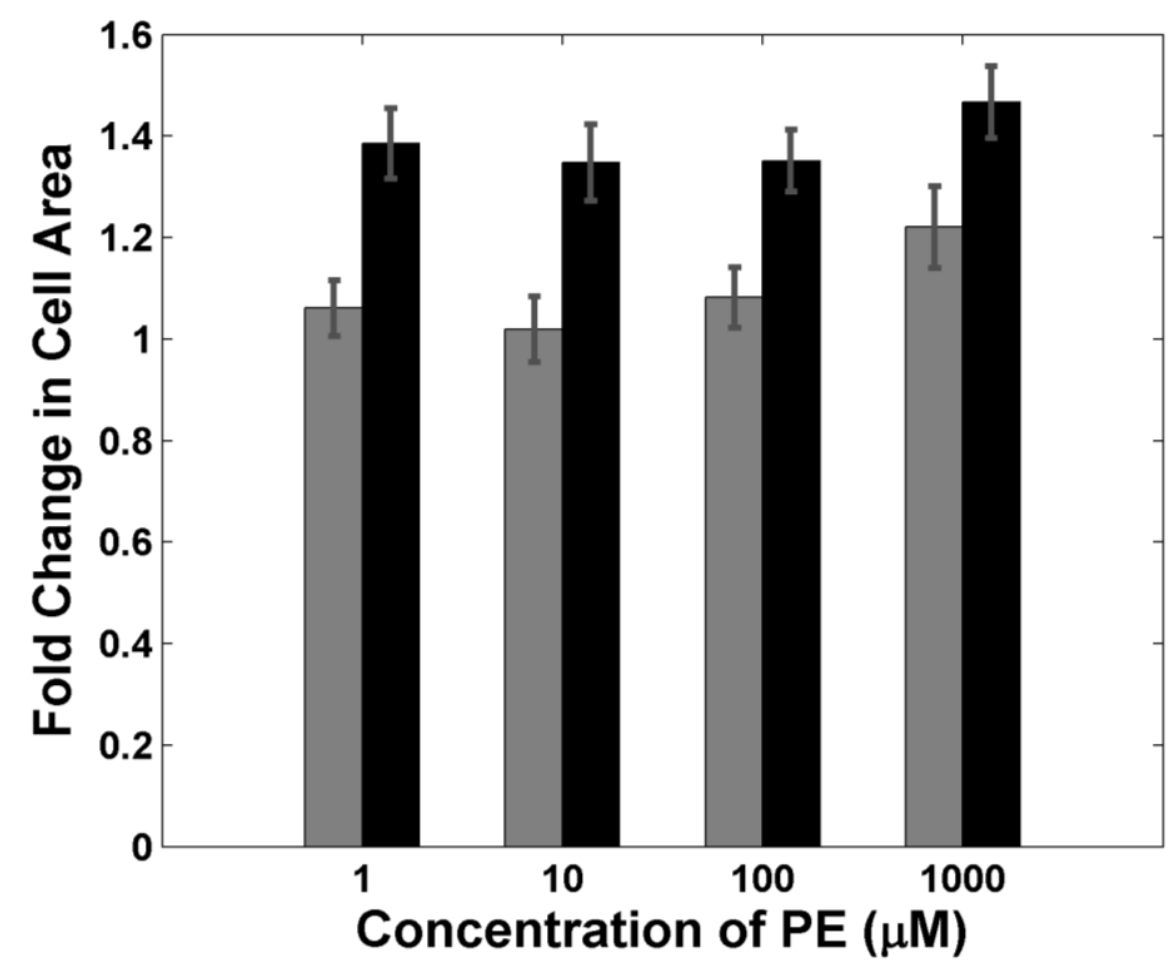

Figure 2.5: Residual PE after washout does not explain reversibility delay. Median fold changes in cell area for myocytes ( $\sim 150$ cells per condition) exposed to a given concentration of PE for 30 seconds (gray bars) or 24 hours (black bars). Images were captured before exposure to PE and 24 hours after initial exposure to PE. Any remaining PE after washout should be independent of time of exposure to the cells. Therefore looking at the 30 second results could give an estimate of the effects of insufficient washout. The hypertrophy response in the 30 second condition is too small compared to the 24 hour response to explain the sustained hypertrophy seen in high PE concentration conditions. The hypertrophy response to 30 seconds of $1 \mathrm{mmol} / \mathrm{L} P E$ is much less than the response to 24 hours of $1 \mu \mathrm{mol} / \mathrm{L}$ response, implying that if any PE is remaining after washout it $<1 \mu \mathrm{mol} / \mathrm{L}$. Moreover, since the 30 -second $1 \mathrm{mM} P E$ response is $\sim 50 \%$ the level of the 24 -hour $1 \mu \mathrm{M}$ PE response, the potential contamination is likely closer to 0.1 $\mu \mathrm{mol} / \mathrm{L}$ PE. From looking at PE data in Figure 2.4A, the projected area increase after 48-hour stimulation of $<1 \mu \mathrm{mol} / \mathrm{LPE}$ is less than the increase in area observed after washout in the $1 \mathrm{mM}$ condition. It appears that less than $1 \mu \mathrm{mol} / \mathrm{L}$ PE remains in the highest concentration tested $(1 \mathrm{mM})$, and $<1 \mu \mathrm{mol} / \mathrm{L}$ residual PE would not be able to produce the magnitude of reversibility delay seen experimentally. These results imply that residual PE after washout is not responsible for the observed reversibility delay. Error bars are +/- SE. 
Integrated fluorescence intensity of the GFP driven by the TnT promoter follows a similar pattern as cell area after exposure to PE (Figure 2.6). Myocytes exposed to the highest concentrations of PE continue to increase in integrated GFP fluorescence after agonist washout at 24 hours, while the lower concentrations begin to decrease in integrated intensity after agonist washout. We performed additional validations to ensure that increases in integrated GFP fluorescence upon application of PE were not affecting myocyte area measurements (Figure 2.7). Integrated GFP fluorescence would not increase if the cells were only spreading out. Thus the integrated GFP fluorescence intensity provides a reporter of troponin T promoter activity that is relatively independent of myocyte growth.

The data show that the kinetics of cardiac myocyte hypertrophy after removal of PE is a concentration dependent reversibility delay: PE-induced hypertrophy is reversible at low concentrations of PE and persists at higher concentrations despite washout of the agonist. This surprising result has implications for a memory response in aAR-mediated hypertrophy. It suggests that hypertrophy may persist for a significant time period after exposure to transient circulating $\alpha A R$ agonists resulting from the neurohumoral response to stress. This memory response could accelerate development of hypertrophy and hinder its reversal.

\subsubsection{Evidence for the role of intracellular $\alpha A R^{\prime}$ s in reversibility delay}

We hypothesized that sustained $\alpha A R$ signaling inside the cell allowed for the continued increase in cell area even after extracellular washout of PE. This could be mediated by cellular uptake of PE. Therefore myocytes given high extracellular concentrations of PE could potentially transport enough PE into the cell during the 24 hour exposure period for sustained increases in cell area. Recent evidence that $\alpha A R^{\prime}$ s colocalize with intracellular endosomes [53] and the nuclear membrane in adult cardiac myocytes [54] provides a potential mechanism for this intracellular $\alpha A R$ signaling. Therefore, we hypothesized that the concentration dependent reversibility delay is explained by intracellular ligand trapping: internalized PE acting on intracellular $\alpha A R^{\prime} s$. 


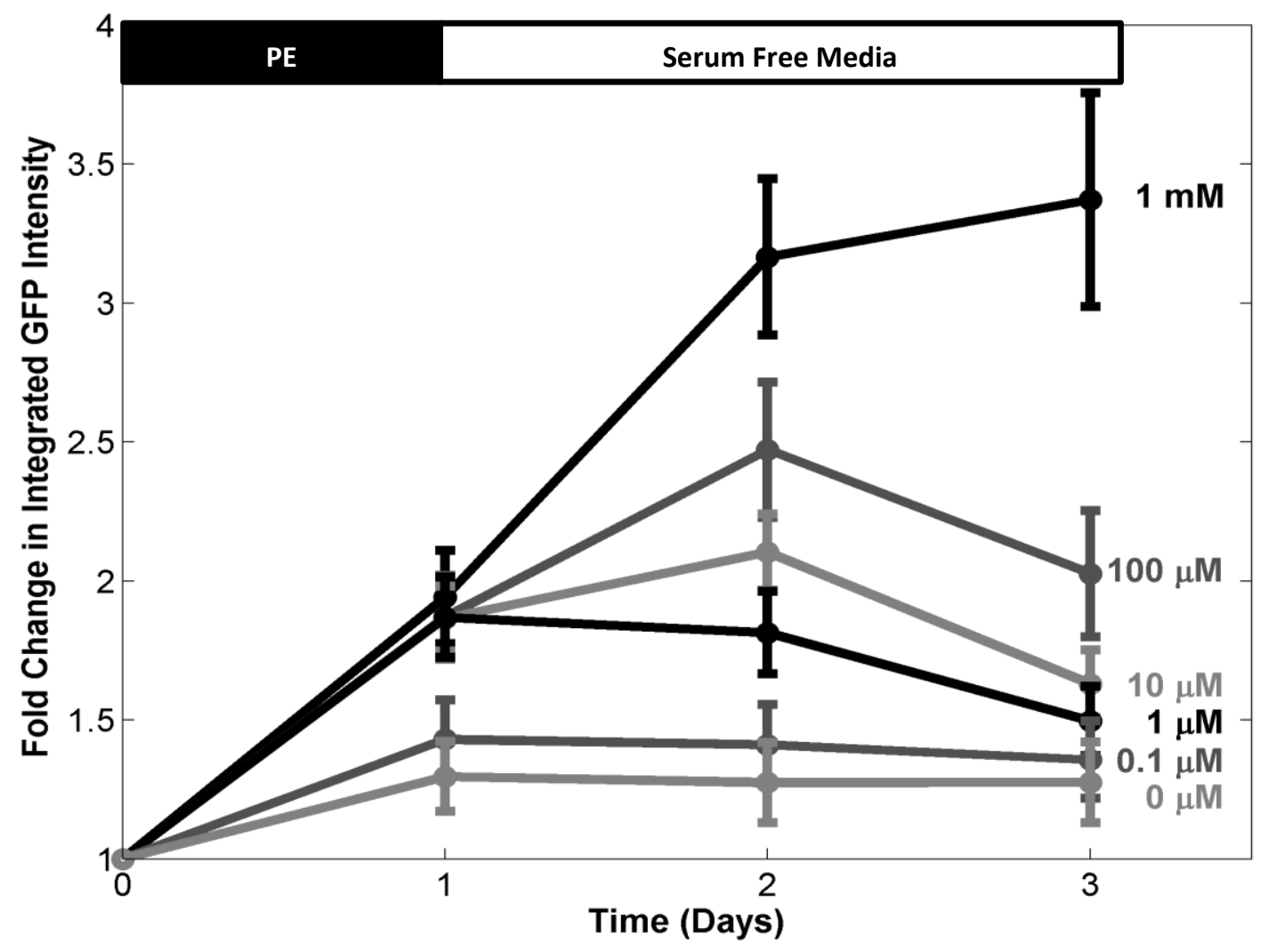

Figure 2.6: Increases in integrated GFP intensity are reversible at low but not high levels of $\alpha$ adrenergic signaling. Time course data of median ( $\sim 00$ cells per condition) fold change in integrated intensity of cardiac myocytes after 24 hours exposure to a given concentration of PE in serum free media. On day 1 the agonist was washed out and replaced with serum free media. Error bars are +/- SE. These results indicate that along with cell area, activity of the troponin T promoter is increasing with addition of increasing amounts of PE and has similar time course trends as cell size. 

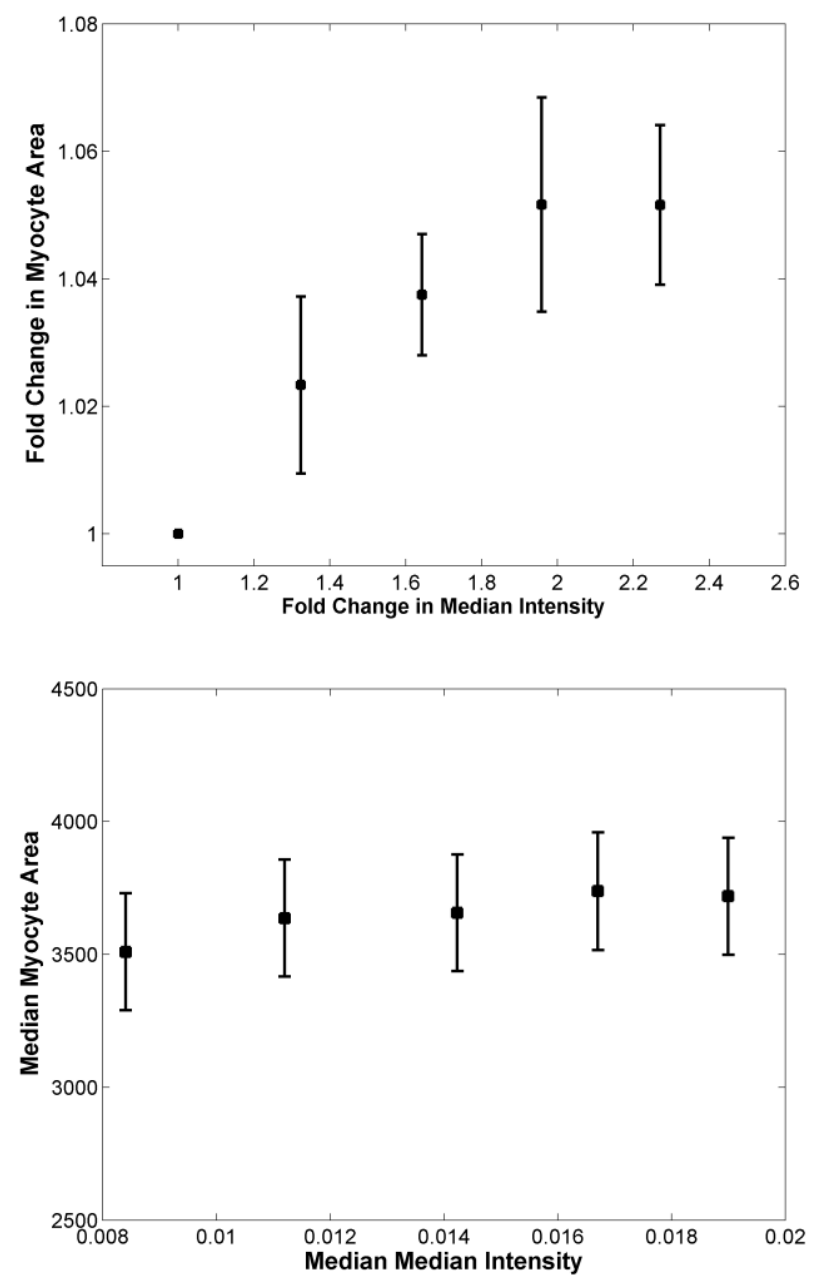

Figure 2.7: Increases in fluorescence intensity of cardiac myocytes have minimal effects on cell area measurements. We imaged the same region of cardiac myocytes at five different exposure times (100, $150,200,250$, and $300 \mathrm{~ms}$ ) to test the effects of increased fluorescence intensity on cell area measurements. Each image was median filtered with a 2.0 radius window to reduce the effects of increased background noise on the segmentation. The change in median intensity of myocytes between the 100 and 300 ms exposure times was similar to the intensity change seen in $1 \mathrm{mM} \mathrm{PE}$ condition between the first and last image collected in the 3 day imaging period used in the hypertrophy reversibility experiments. a) Median fold change in myocyte area given the median fold change in median intensity seen with each incremental increase in exposure time. $\mathrm{N}=139$ cells per condition and error bars are +/- SE. The fold change in myocyte area leveled off at around $5 \%$ for the maximum intensity increases recorded in our experiments. This $5 \%$ increase in area is much less than the area increases we were observing with addition of PE and is therefore an acceptable level of error. b) Median myocyte area and the corresponding median median cell pixel intensity at each incremental increase in exposure time. $\mathrm{N}=139$ cells per condition and error bars are +/- SE. The changes in median pixel intensity observed between the lowest and highest exposure times were comparable with the maximum increases in median cell pixel intensity observed when adding the highest concentrations of PE to the myocytes. With increasing median pixel intensities the corresponding increases in cell area began leveling off. 
To test this hypothesis, two specialized $\alpha A R$ antagonists were used: prazosin, an $\alpha A R$ antagonist that can act at the sarcolemma and also be transported inside the cell, and CGP-12177a, a hydrophilic $\alpha A R$ antagonist that cannot be internalized and therefore acts only at the sarcolemma[55-58]. After recording initial images, a given concentration of prazosin or CGP-12177a with $10 \mu \mathrm{mol} / \mathrm{L}$ PE was applied to the cardiac myocytes for 24 hours (Figure 2.8A). Myocytes cultured with prazosin showed concentration dependent prevention of hypertrophy. Conversely, CGP-12177a did not prevent PEinduced myocyte hypertrophy. In other words, an $\alpha A R$ antagonist that can act inside the cell was able to prevent PE-induced hypertrophy and $\alpha A R$ antagonist that can only act at the sarcolemma was not. These results imply that $\alpha A R^{\prime} s$ inside the cell are involved in PE-induced hypertrophy, which is consistent with our ligand trapping hypothesis.

To further test this mechanism, $10 \mu \mathrm{mol} / \mathrm{L}$ prazosin or CGP-12177a in serum free media was administered to the myocytes after 24 hour exposure to a given concentration of PE (Figure 2.8B). Myocytes treated with prazosin began decreasing in area immediately after PE washout at all concentrations of PE. This provides evidence that continued increases in cell area after extracellular PE washout was due to sustained $\alpha A R$ activation. The reversibility data with prazosin indicate that the largest contributor of the reversibility delay is at the level of the $\alpha A R$ and not due to feedback downsteam of $\alpha A R^{\prime}$ s such as from autocrine or paracrine effects [59] or nonspecificity of PE. Moreover, reversibility kinetics with prazosin compared to untreated (Figure 2.4) imply that expression of GFP did not substantially affect reversal of myocyte growth.

Conversely, myocytes given CGP-12177a continued to exhibit a reversibility delay response, where hypertrophy was reversible at low $(\mathrm{PE} \leq 10 \mu \mathrm{mol} / \mathrm{L})$, but not high levels of alpha-adrenergic signaling. The final fold changes in cell area observed on day 3 with CGP-12177a were somewhat higher than what was seen in the serum-free media only condition. Based on the time course of the $0 \mu \mathrm{mol} / \mathrm{L}$ PE myocytes, CGP-12177a induces minor increases in cell area on its own consistent with its mild partial 

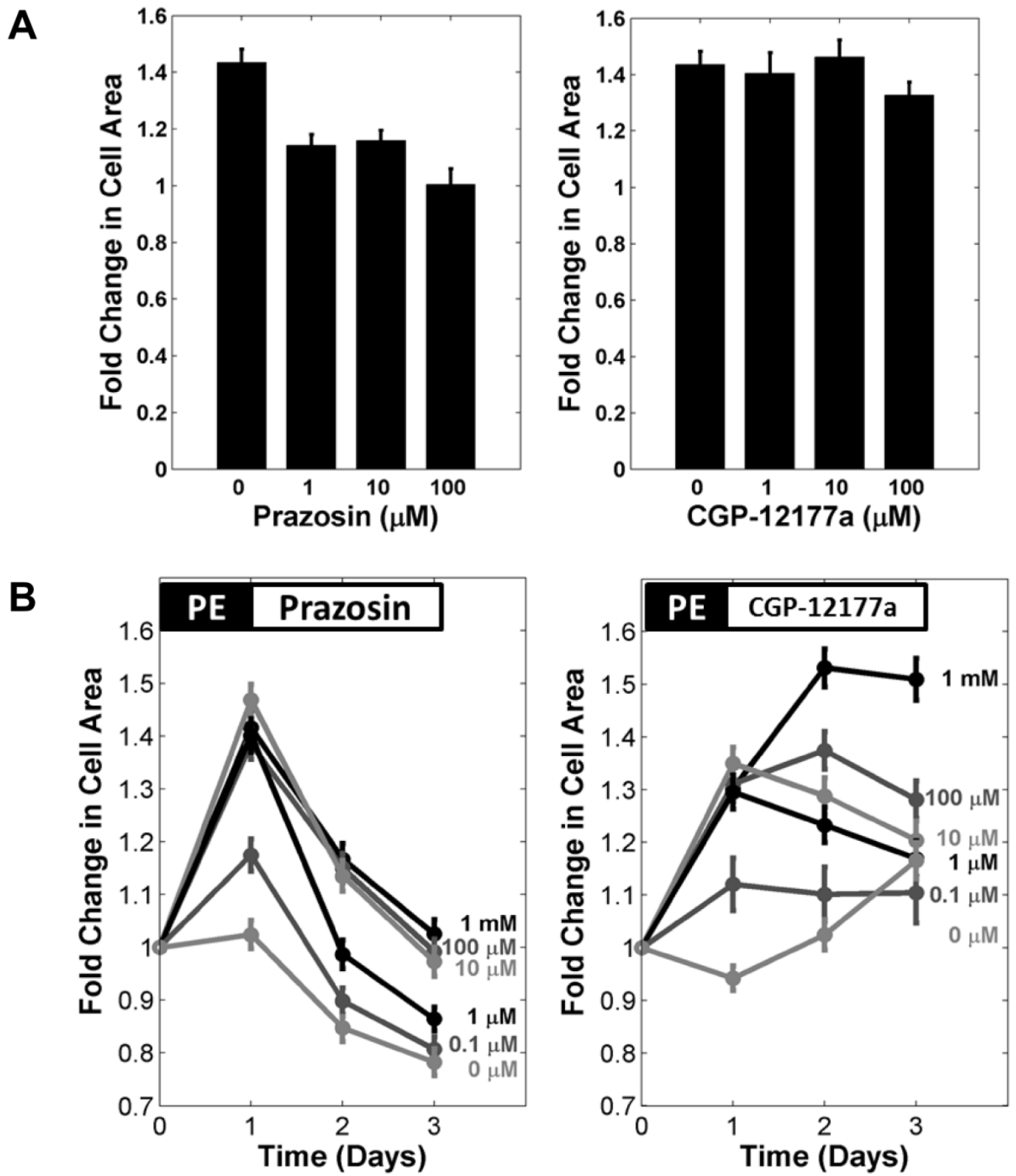

Figure 2.8: Hypertrophy is reversed by membrane permeable $\alpha A R$-antagonist but not membrane impermeable antagonist. A) Median ( 350 cells per condition) fold change in cell area of cardiac myocytes exposed to $10 \mu \mathrm{mol} / \mathrm{L}$ of PE with a given concentration of prazosin (left) or CGP-12177a (right) for 24 hours. prazosin and CGP-12177a are both alpha adrenergic receptor antagonists. Prazosin can act at the sarcolemma and also be transported inside the cell and CGP-12177a can only act at the sarcolemma. Error bars are +/- SE. B) Time course data of median ( 1000 cells per condition) fold change in cell area of cardiac myocytes after 24 hours exposure to a given concentration of PE. On day 1, PE was washed out and cells were cultured in $10 \mu \mathrm{mol} / \mathrm{L}$ prazosin (left) or $10 \mu \mathrm{mol} / \mathrm{L} \mathrm{CGP-12177a} \mathrm{(right)} \mathrm{in}$ serum free media. Error bars are +/- SE. Prazosin reverses PE-induced hypertrophy. CGP-12177a does not affect hypertrophy reversibility, supporting the hypothesis that internalized PE acting on nuclear $\alpha A R^{\prime} s$ may explain the reversibility delay. 
A

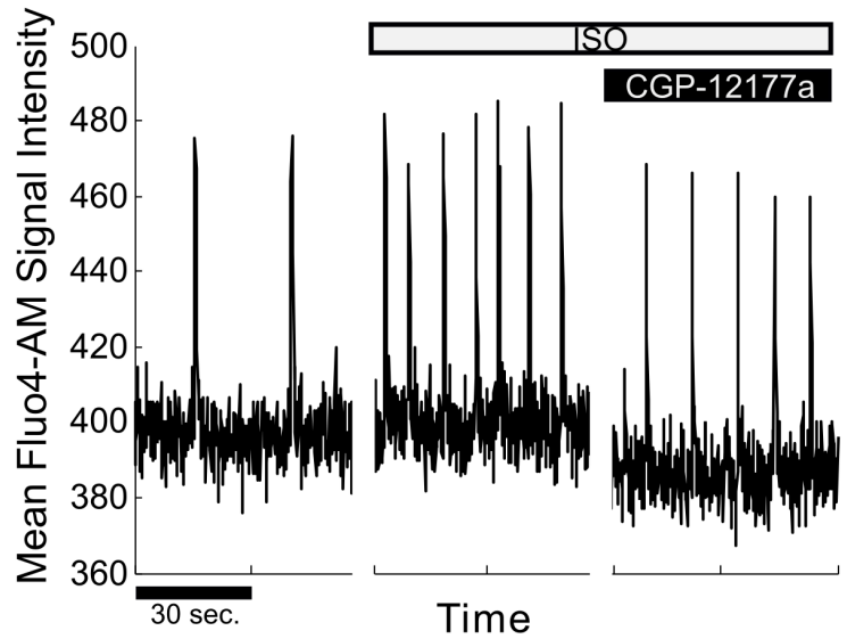

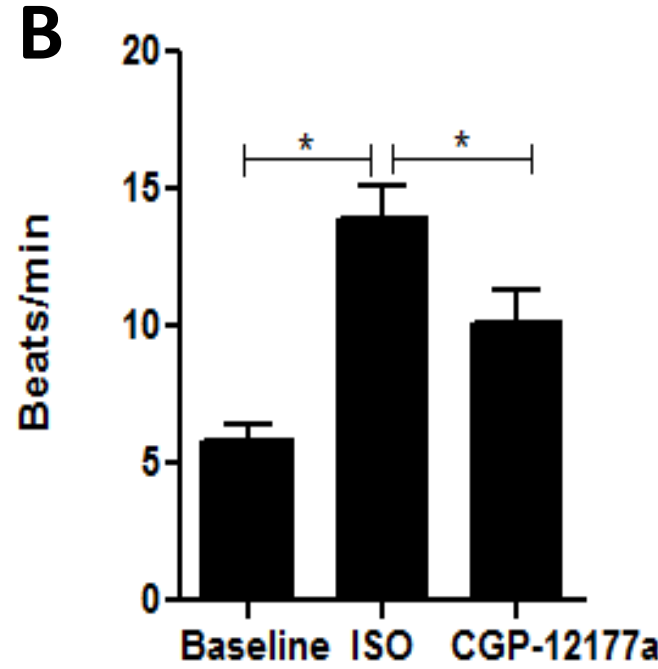

Figure 2.9: CGP-12177a is a $\boldsymbol{\beta} 1$-adrenergic receptor antagonist. To show that the CGP-12177a used in reversibility experiments was active, we imaged calcium transients in cultured neonatal rat ventricular myocytes to demonstrate its ability to act as a $\beta 1$-adrenergic receptor antagonist. Myocytes were loaded with Fluo-4AM (Invitrogen) and calcium transients were imaged for a total of 6 minutes. Baseline calcium transients were recorded for 2 minutes, then $10 \mathrm{nmol} / \mathrm{L} \beta 1$-adrenergic receptor agonist isoproterenol (ISO) was applied to the myocytes. Transients were recorded for another 2 minutes before applying $100 \mu \mathrm{mol} / \mathrm{L} \mathrm{CGP-12177a}$ to the solution and recording transients for 2 additional minutes. A) Representative mean Fluo4-AM signal intensity over time for a myocyte during the three conditions tested. Each interval shown is one minute, time scale bar is 30 seconds. Beating frequency increased with isoproterenol and decreased with CGP-12177a. B) The number of beats/minute was calculated for each myocyte $(\mathrm{N}=9)$ for the second minute of data collection for each condition to give myocytes time to respond to the stimulus. Results showed that isoproterenol significantly increased the number of beats per minute and CGP-12177a significantly reduced the number of beats $/ \mathrm{min}$. Differences in beats $/ \mathrm{min}$ were tested for statistical significance using a one way ANOVA with Bonferroni's multiple comparison post-test. ${ }^{*} \mathrm{P}<.05$. 
agonism towards $\beta 3$-adrenergic receptors [56] and also providing evidence that the CGP-12177a used in these experiments was active. We further demonstrate activity of CGP-12177a by demonstrating that the compound acts as a $\beta 1$-adrenergic receptor antagonist [58] (Figure 2.9). Together these results support the hypothesis that the concentration dependent reversibility delay may be explained by intracellular ligand trapping: cellular uptake of PE with hypertrophy induced by activity at intracellular $\alpha A R^{\prime} s$.

\subsubsection{Mathematical model of ligand trapping explains hypertrophy reversibility delay}

To further evaluate ligand trapping's role in the reversibility of PE-induced hypertrophy, we developed an ordinary differential equation model of PE-internalization and myocyte hypertrophy (Figure 2.10A). In the model, cellular uptake rate of PE is linearly related to the extracellular PE concentration. After internalization, PE can act on intracellular $\alpha A R^{\prime} s$ and lead to increased cell area. Both PE degradation and activity at $\alpha A R^{\prime} s$ are modeled using saturating, Michaelis-Menten form kinetics. Cell area is also influenced by constant basal growth and linear atrophy terms. Equations are provided in Appendix B.

Model parameters were estimated by nonlinear least-squares fitting to experimental data from Figure 2.4A. Figure 2.10B shows that the model predictions closely fit the experimental data on reversibility of PE-induced hypertrophy. As we saw experimentally, the ligand trapping model predicts the reversibility delay response, with persisting hypertrophy at high concentrations of PE. The model output shows how PE internalization allows for the time of the peak fold change in cell area to shift to later time points when myocytes are exposed to higher extracellular concentrations of PE.

The ligand trapping model makes predictions of how intracellular PE concentration and fraction of ligand-bound $\alpha A R^{\prime} s$ (Figure 2.10C) elicit sustained hypertrophic responses. After extracellular PE washout at 24 hours, internal PE concentration remains high until PE degradation reduces it back to 

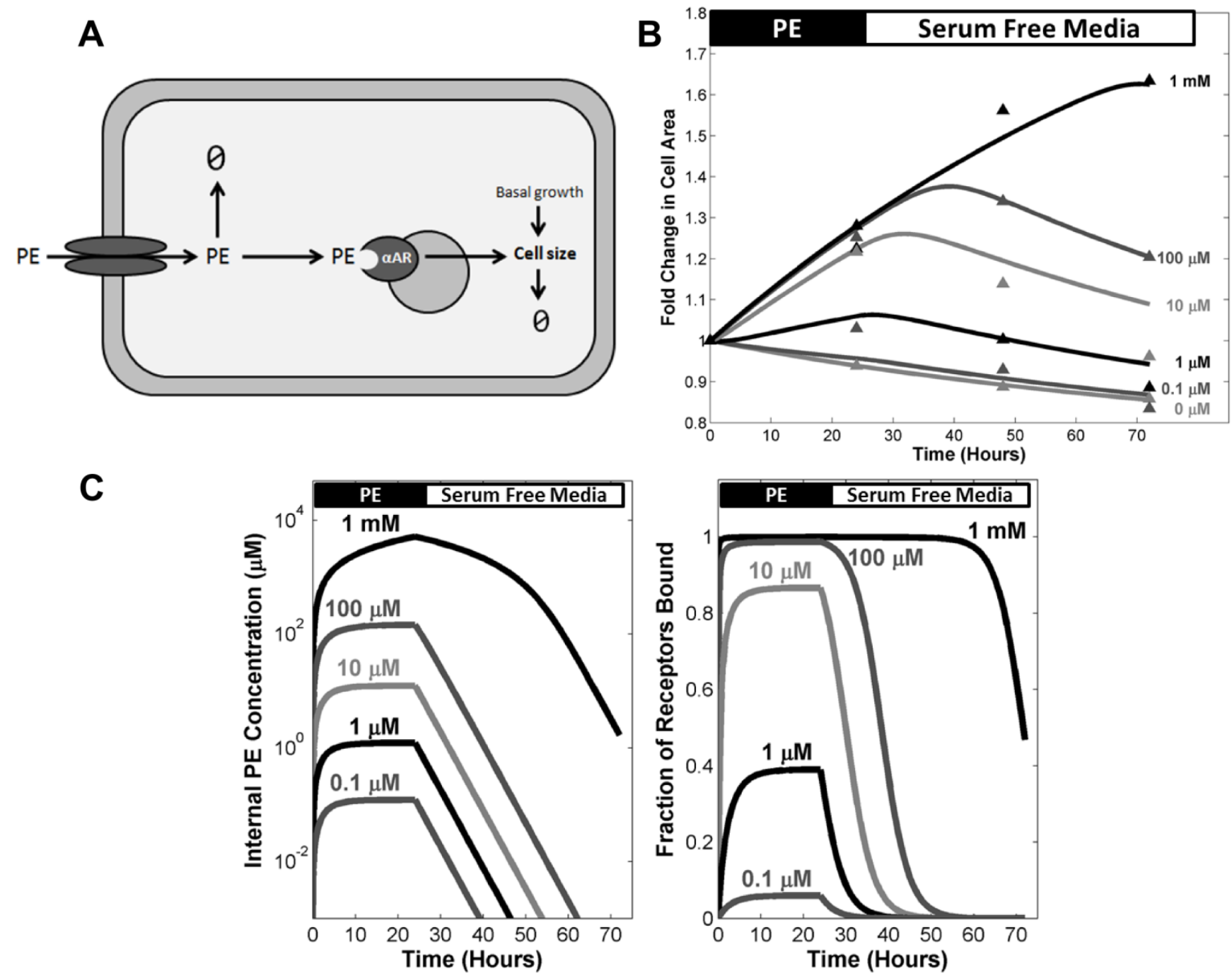

Figure 2.10: Concentration-dependent reversibility delay may be explained by a ligand trapping model. A) Schematic of the mathematical model. PE is internalized by the cell and acts on intracellular $\alpha A R^{\prime} s$, which results in increased cell size. B) Results of nonlinear least squares fit of the model to experimental data (points). C) Model-predicted time courses for internal PE concentration (left) and fraction of $\alpha A R^{\prime} s$ with bound PE (right). At high concentrations, internal PE concentration and fraction of receptors bound remains high after agonist is washed out of the extracellular media, allowing for further increases in myocyte hypertrophy. 


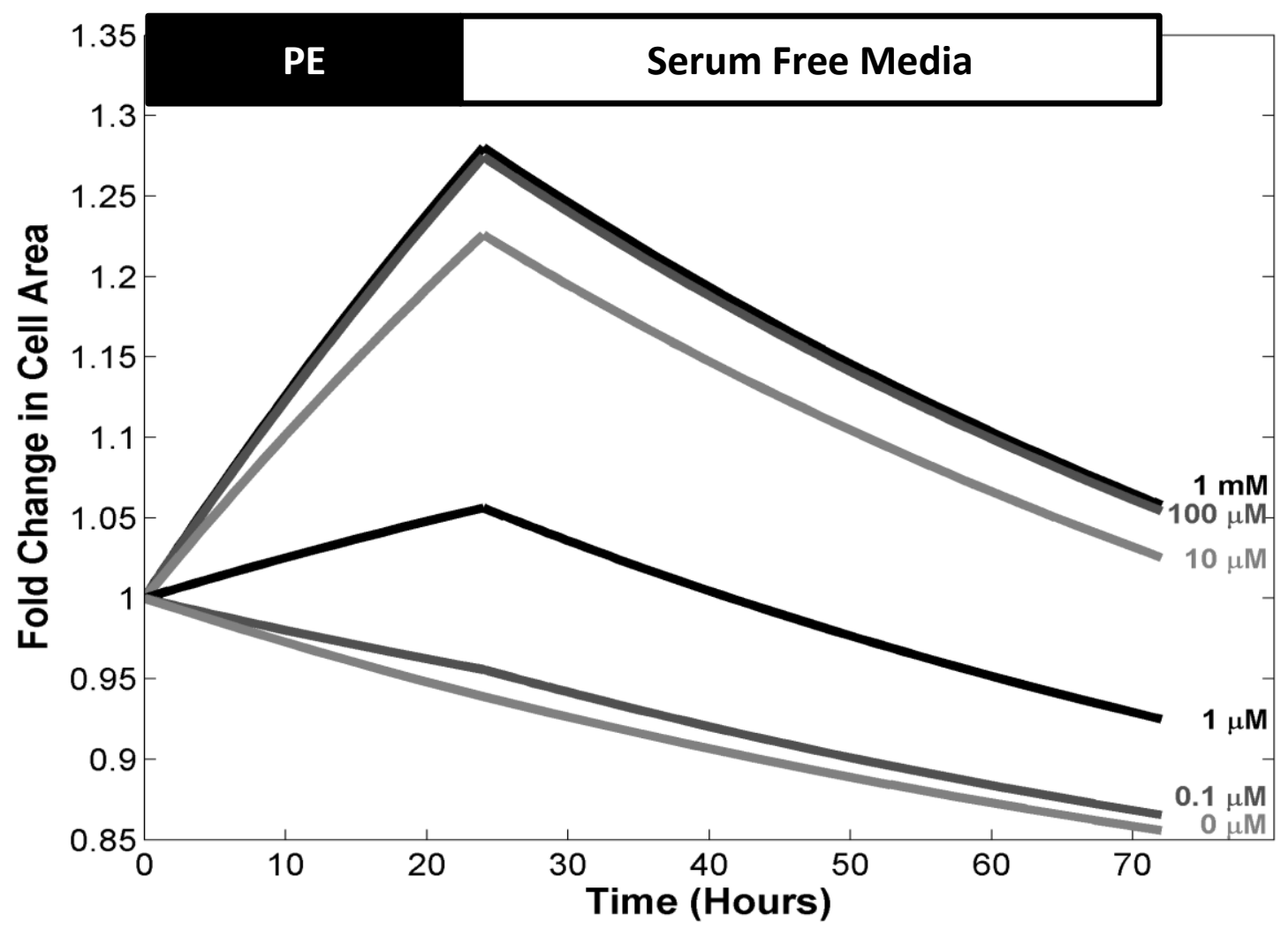

Figure 2.11: A model containing only sarcolemmal $\alpha A R^{\prime} s$ cannot reproduce the experimentally observed reversibility delay. Model predictions of fold change in cell size after exposure to a given concentration of PE of myocytes with only sarcolemmal $\alpha A R$ 's. Unlike experimental observations, this model is not able to generate sustained increases in cell size after washout of the agonist at any tested concentration of PE. 


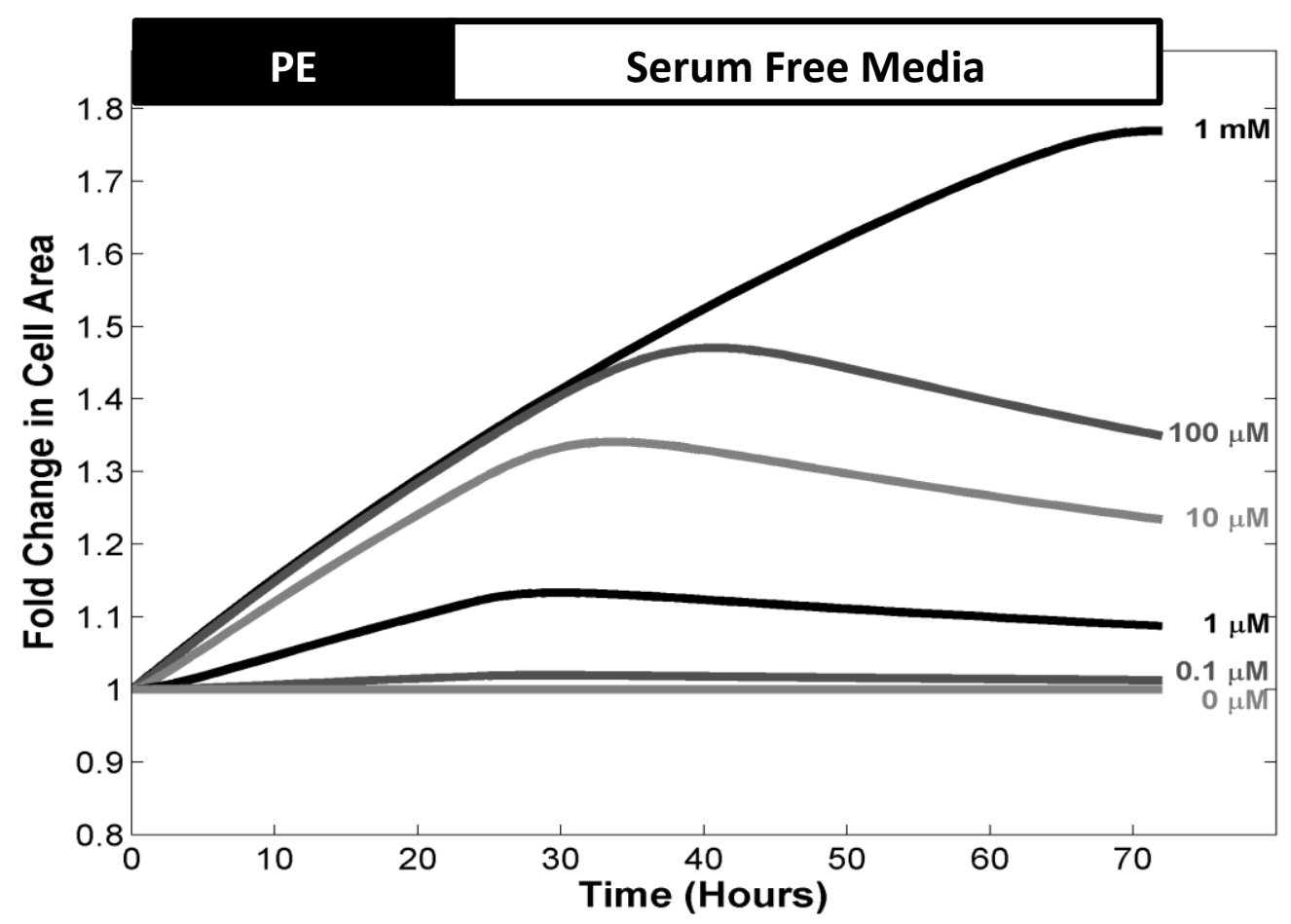

Figure 2.12: A basal growth term is required in the model to reproduce experimental observations of decreases in myocyte size below initial value. Model predictions of fold change in cell size after exposure to a given concentration of PE of myocytes without a basal growth term affecting cell size. Unlike experimental observations, in this version of the ligand trapping model, cell size cannot fall below the initial observation. However, this version of the model does still exhibit a concentration-dependent reversibility delay in hypertrophy. 


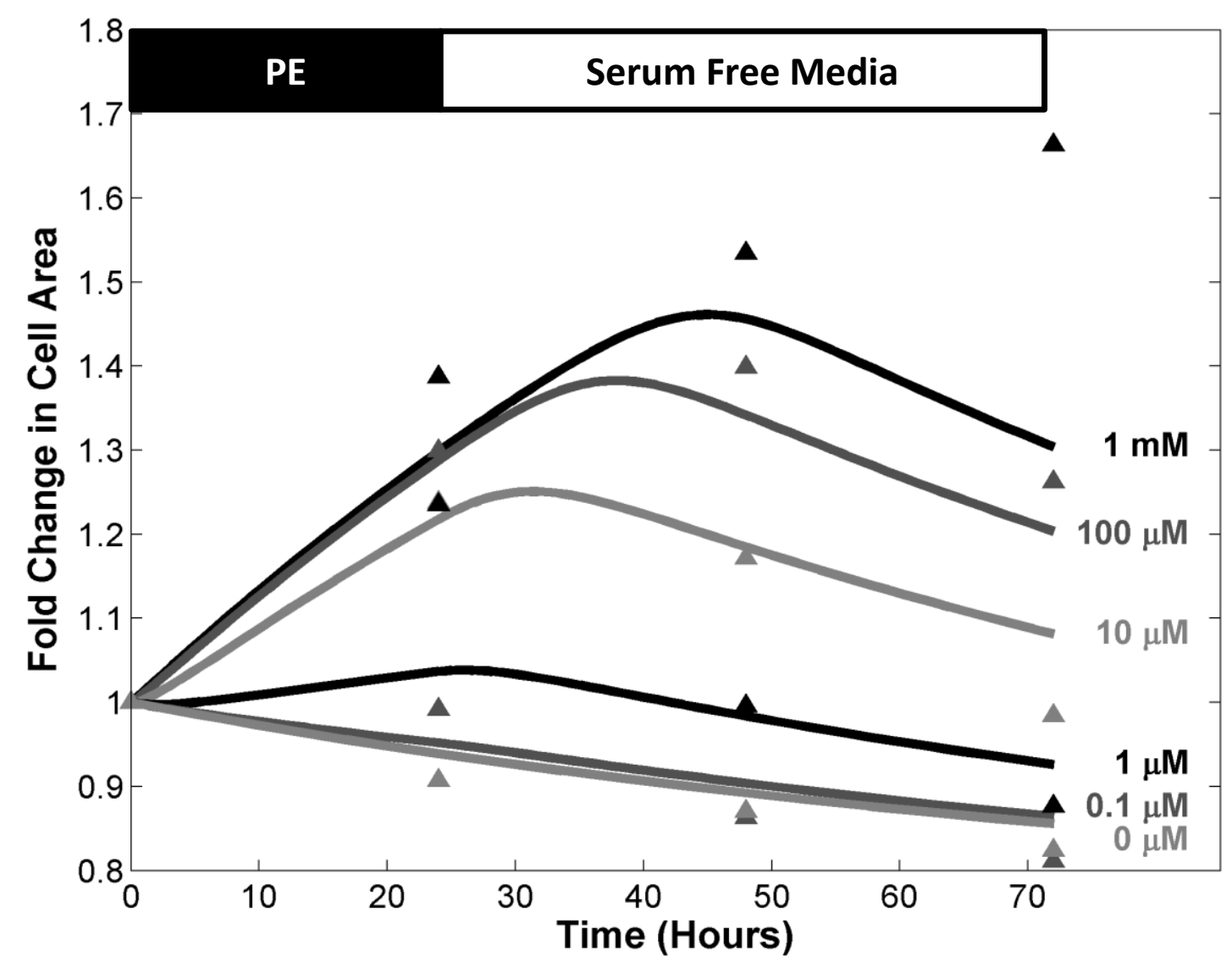

Figure 2.13: Saturation of degradation of internalized PE is required to fit $1 \mathrm{mmol} / \mathrm{L} \mathrm{PE}$ data. Nonlinear least squares fit of ligand trapping model with linear degradation of internalized PE and experimental data (points). This model structure is not able to generate the sustained growth of the myocytes exposed to $1 \mathrm{mmol} / \mathrm{L}$ PE seen 48 hours after washout of PE. 


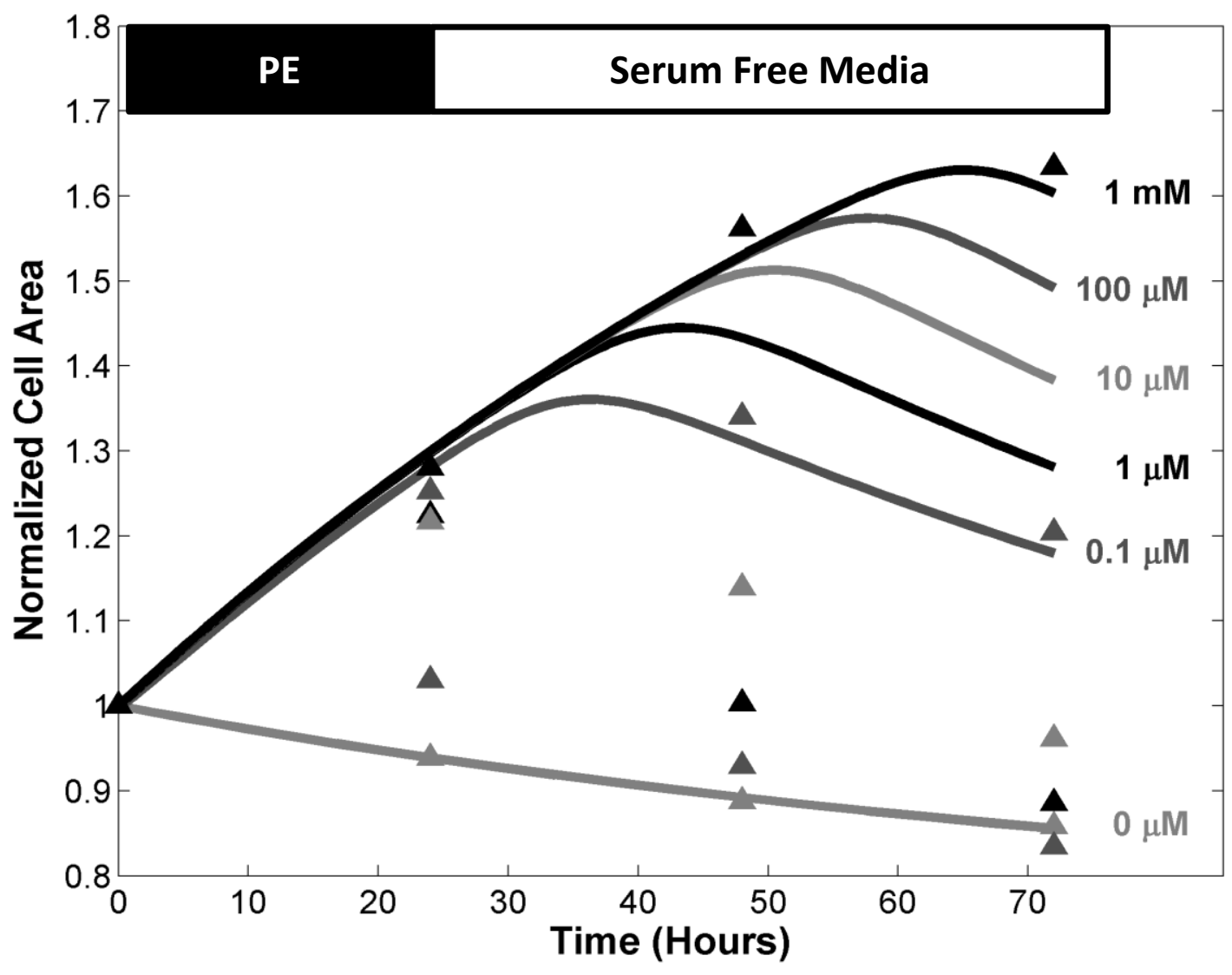

Figure 2.14: Saturation of degradation of internalized $P E$ is required to fit across all PE concentrations tested. Experimental data for $1 \mathrm{mmol} / \mathrm{L} \mathrm{PE}$ treated myocytes can also be fit by increasing the internalization parameter k1 from .2447 hours $^{-1}$ to 145 hours $^{-1}$. The model results compared to experimental data (points) of this fit are shown in the plot above. Increased rate of internalization of PE resulted in a good fit for $1 \mathrm{mmol} / \mathrm{LPE}$, but a poor fit for each of the other concentrations tested. Therefore it would be possible to fit the data with a nonlinear (e.g. quadratic) equation for uptake of PE, but a three-fold increase in uptake at high extracellular concentrations does not make sense biologically. Therefore, we changed degradation of PE to be saturating and hypothesize that there may be a transport of enzyme mediated degradation that would cause this saturation. 
baseline. The higher the extracellular concentration of PE myocytes were exposed to, the longer the time internal PE remains high enough to continue to act on intracellular $\alpha A R^{\prime} s$ to increase myocyte area.

The $1 \mathrm{mmol} / \mathrm{L}$ PE degradation kinetics appear distinct from the lower concentrations due to the saturating degradation of PE. Similarly, since internal levels of PE persist after washout, the fraction of receptors bound remains high for longer in myocytes exposed to higher extracellular concentrations of PE. Intracellular $\alpha A R^{\prime} s$, basal cell growth, and saturating PE degradation were all required to reproduce experimental observations (Figure 2.11-2.14). The latter may imply that an enzyme or transport mediated process is responsible for decreases in the intracellular PE over time.

A key test of this model is its ability to predict the response to prazosin (data from Figure 2.8B), which was not used in model construction. Prazosin was modeled as a competitive inhibitor with $\mathrm{K}_{\mathrm{i}}$ of $0.1 \mathrm{nM}[60]$. As seen experimentally, the model showed reversibility of PE-induced hypertrophy with addition of prazosin at all PE concentrations (Figure 2.15). Experimental data for prazosin experiments had higher peak fold changes, but the reversibility kinetics for the model and experimental data are qualitatively similar. Moreover, the model is able to accurately predict longer time course data for the 1 $\mathrm{mmol} / \mathrm{L}$ PE condition, which was also not used in model construction. In the 4-day time window of data collection from experiments shown in Figure 2.10, the $1 \mathrm{mmol} / \mathrm{L}$ PE condition appeared irreversible, while the model structure predicted it would eventually return to baseline, only at a later time point than the lower PE concentrations. In subsequent experiments, myocytes in the $1 \mathrm{mmol} / \mathrm{L} \mathrm{PE}$ condition began to decrease in cell area 48 hours after PE washout, with kinetics remarkably similar to the prior model predictions (Figure 2.16). These results are consistent with the ligand trapping hypothesis, that the reversibility delay in PE-induced hypertrophy is a result of internalized PE acting on intracellular $\alpha A R^{\prime} s$. 


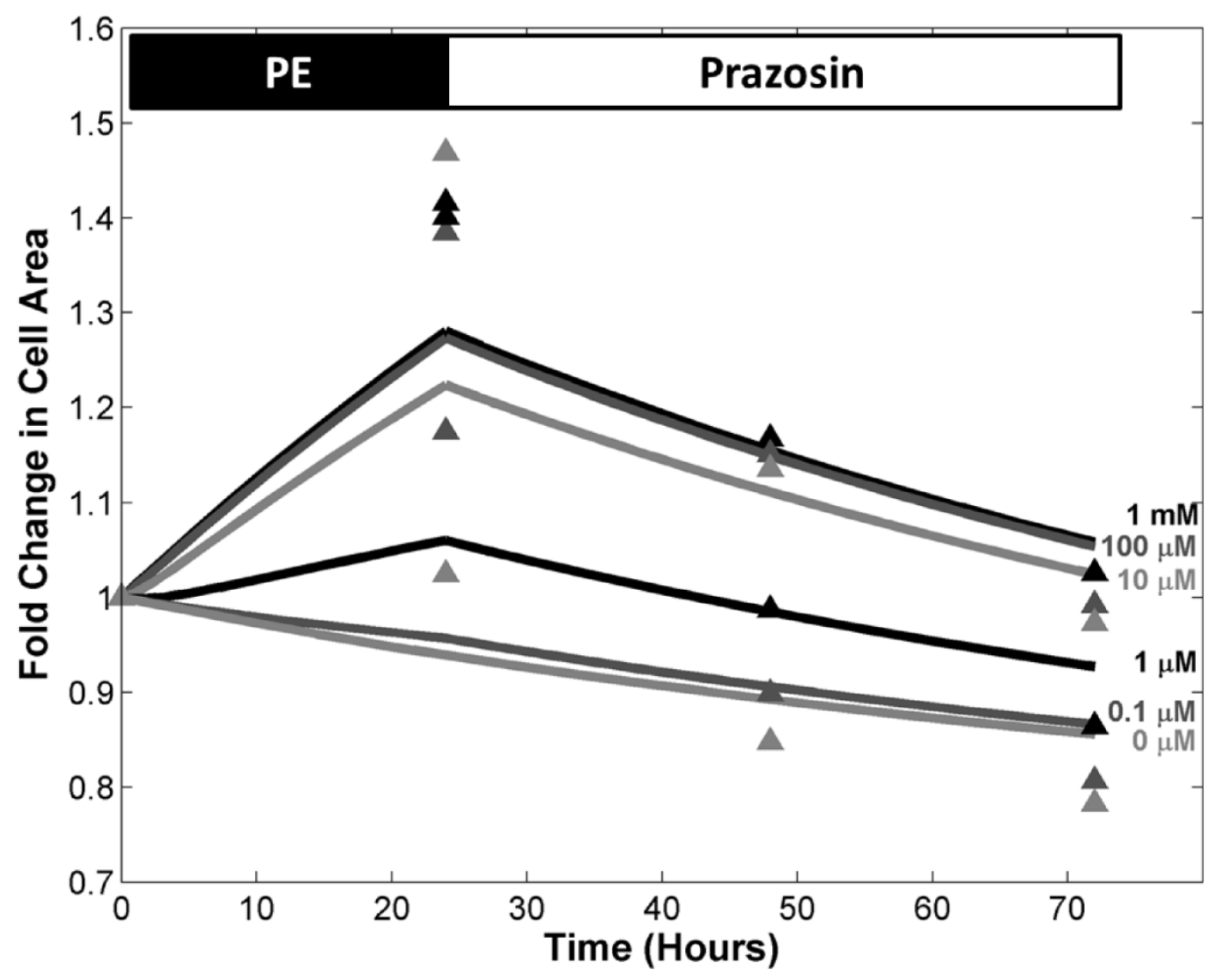

Figure 2.15: Ligand trapping model predicts reversibility of hypertrophy with prazosin and longer time course data of $1 \mathrm{mmol} / \mathrm{L} \mathrm{PE}$ treated myocytes. A) Mathematical model predictions and experimental data (points) of fold change in cell size of myocytes exposed to PE and then prazosin after agonist washout. As seen experimentally, the model predicts that hypertrophy is reversed by membrane permeable $\alpha A R$ antagonist, prazosin $\left(\mathrm{K}_{\mathrm{i}}=0.1 \mathrm{nM}\right)$, after 24 hour exposure to a given concentration of $\mathrm{PE}$. 


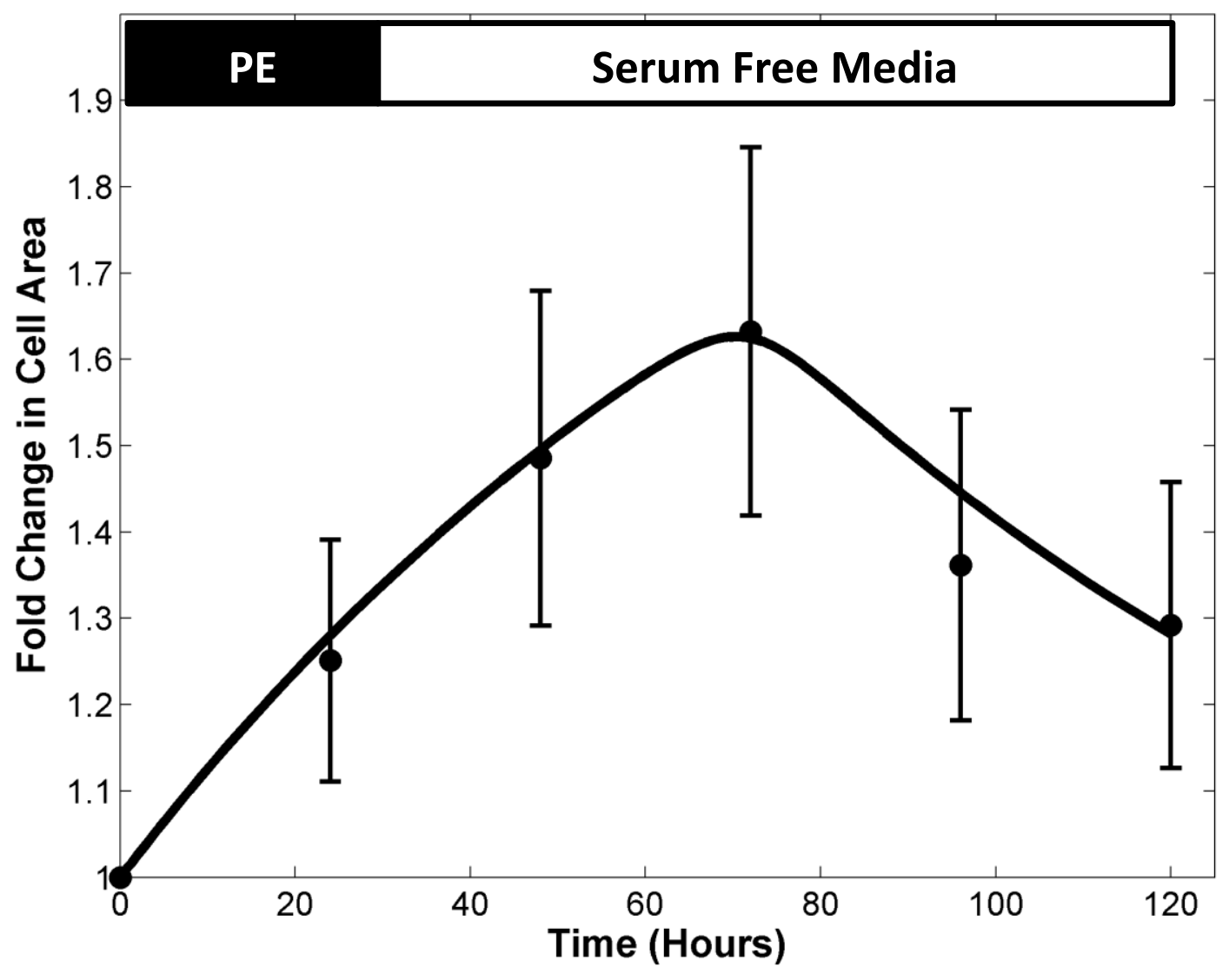

Figure 2.16: Ligand trapping model predicts longer time course data of $1 \mathrm{mmol} / \mathrm{L}$ PE treated myocytes. Model predictions and experimental data (61 cells) of fold change in cell size of myocytes exposed to 1 $\mathrm{mmol} / \mathrm{L}$ PE for 24 hours. Ligand trapping model predicts that 48 hours after agonist washout, myocytes exposed to $1 \mathrm{mmol} / \mathrm{L}$ PE will begin to decrease in cell size. This is consistent with longer time course experimental data, which was not used to fit the model. Error bars are +/- SE. 


\subsection{4 $\alpha A R$ localization determined using BODIPY-prazosin is consistent with ligand trapping model}

Our ligand trapping mathematical model predicts that intracellular $\alpha A R^{\prime} s$ are necessary for the concentration-dependent reversibility delay in PE-induced hypertrophy. To test this prediction we collected confocal microscopy images of myocytes with BODIPY-FL fluorescently labeled prazosin to determine the distribution of $\alpha A R^{\prime} s$ in cardiac myocytes. BODIPY-prazosin fluoresces when bound to $\alpha A R^{\prime} s$ and remains effectively non-fluorescent when unbound [61]. Images revealed that the majority of BODIPY-prazosin labeling was present inside the cardiac myocytes (Figure 2.17A). Quantification of labeling from a 3D stack of images throughout the entire myocyte offer further evidence that most of the $\alpha A R^{\prime} s$ were located in the cell interior and not the sarcolemma (Figure 2.17B). The distribution of BODIPY-prazosin labeling within the cells was punctate in appearance, resembling vesicles. Moreover, time-lapse images show directed movement of these punctate structures, which is consistent with the expected motion of vesicular trafficking. This vesicular arrangement of $\alpha A R^{\prime} s$ is consistent with previous data that showed that expressing $\alpha_{1 a} A R^{\prime}$ s in R-1F cells were predominately found in intracellular organelles, including early and late endosomes [53]. These punctate vesicular structures are also apparent in BODIPY-prazosin images from adult mouse cardiac myocytes [54]. This result provides evidence of a large population of $\alpha A R^{\prime} s$ inside cardiac myocytes and is therefore consistent with the ligand trapping hypothesis.

Competitive inhibition experiments with phentolamine (an $\alpha A R$ antagonist) and PE were performed to quantify nonspecific binding (Figure 2.17C-D). Labeling was quantified by calculating the integrated intensity of pixels above a threshold (three times the background signal) for each cell and normalizing by cell area. Phentolamine and PE both resulted in significant attenuation of the BODIPYprazosin signal in the cardiac myocytes $(\mathrm{P}<0.001)$. These results confirm the specificity of BODIPYprazosin for $\alpha A R$ labeling and show that PE is internalized by cardiac myocytes and can bind to intracellular $\alpha A R^{\prime}$ 's. CGP-12177a did not significantly attenuate the BODIPY-prazosin labeling inside the 
A
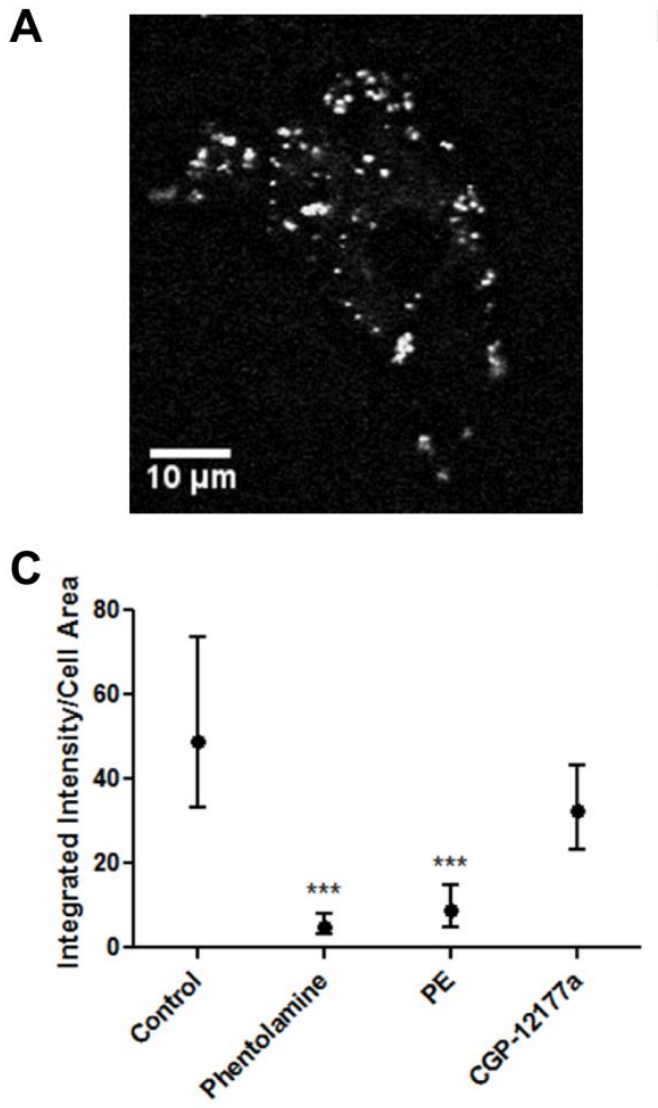

B

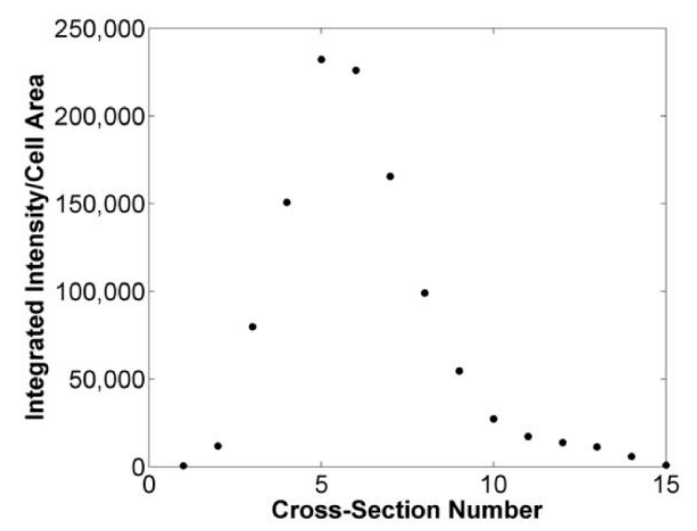

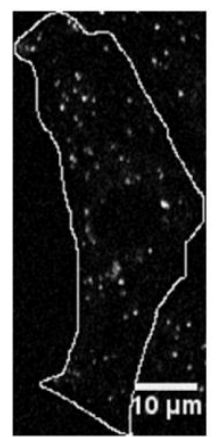

Control: 57.5

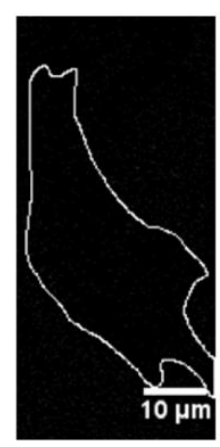

Phentolamine: 4.5

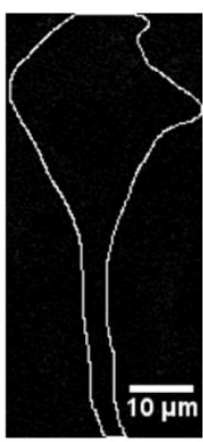

PE:

8.5

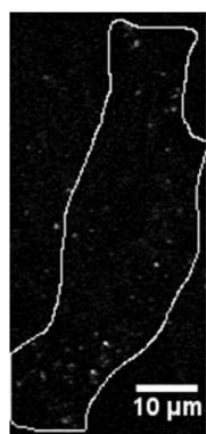

CGP- 12177a: 31.3

Figure 2.17: Intracellular $\alpha A R^{\prime} s$ in cardiac myocytes imaged using fluorescently labeled BODIPYprazosin. A) Cardiac myocytes in a $10 \mathrm{nmol} / \mathrm{L}$ solution of BODIPY-prazosin were imaged using a confocal laser scanning microscope and a $63 \mathrm{X}$ oil objective. The distribution of $\alpha A R^{\prime} \mathrm{s}$ in the myocytes is predominately intracellular, as predicted by the ligand trapping model. The $\alpha A R$ labeling occurred in punctate vesicles spread throughout the cytoplasm. B) 3D Distribution of BODIPY-prazosin labeling indicates large population of intracellular $\alpha A R^{\prime} s$. Integrated intensity of above threshold signal in the BODIPY-prazosin channel normalized by cell area was computed at $0.5-\mu \mathrm{m}$ intervals throughout cardiac myocytes. Results for a representative cell are shown above. Labeling is highest in the center indicating that the majority of $\alpha A R^{\prime} s$ are intracellular, which is consistent with the ligand trapping model. C) Median and interquartile range of integrated intensity of above background BODIPY-prazosin signal normalized by cell area for competitive binding experiments of $10 \mathrm{nmol} / \mathrm{L}$ BODIPY-prazosin with 10 $\mu \mathrm{mol} / \mathrm{L}$ phentolamine, $1 \mathrm{mmol} / \mathrm{L}$ PE, or $100 \mu \mathrm{mol} / \mathrm{L}$ CGP-12177a. N 80 cells per condition. Results with $P E$ and phentolamine show high specificity of BODIPY-prazosin in labeling $\alpha A R^{\prime} s$ and results with CGP12177a provide further evidence that CGP-12177a is not internalized like the $\alpha A R$ antagonist prazosin. $* * * P<0.0001 \mathrm{D})$ Representative images with segmented cell boundaries from the BODIPY-prazosin competitive binding experiments for each condition. The quantification of the labeling (integrated intensity/cell area) for the pictured cell is indicated below. 
cell, providing additional evidence that CGP-12177a is not internalized by the cardiac myocytes like the $\alpha A R$ antagonist prazosin. These results provide evidence of intracellular $\alpha A R^{\prime} s$ and internalization of PE by cardiac myocytes and therefore support our ligand trapping model that the reversibility delay in PEinduced hypertrophy is a result of internalized PE acting on intracellular $\alpha A R^{\prime}$ s.

\subsection{Discussion}

Automated methods that can quickly and reproducibly collect data over spatial and temporal dimensions are needed to study the complex signaling networks controlling cardiac hypertrophy. Here, we developed what is to our knowledge the first automated live-cell image acquisition and analysis approach for high-content imaging of cardiac myocyte hypertrophy. While this method has limited ability to visualize multiple proteins simultaneously or sarcomere striations compared to immunofluorescence techniques, it does not require fixation of the myocytes which allows for multiple time point imaging. Therefore, cardiac myocyte hypertrophy can be quantified and tracked in individual cells over several days. Additionally, even though plasmid-based transfection efficiency does not allow for visualization of every cell in the imaging area, it makes the cell boundaries more conspicuous, which simplifies cell boundary segmentation.

Compared to manual image collection, our method offers substantial increases in imaging speed and reproducibility, increased size of imaging area and therefore a greater number of expressing cells imaged per well/perturbation, and elimination of data collection biases in user-selected regions of cells. With our automated image analysis algorithm, we are able to quickly and robustly quantify features of cell morphology in the large image sets produced using our automated imaging system. Since the algorithm can track myocytes between subsequent images collected over multiple days, we uniquely applied this platform to study reversibility of cardiac myocyte hypertrophy.

Our approach revealed that the kinetics of PE-induced hypertrophy after PE washout is a concentration-dependent reversibility delay: hypertrophy is reversible at low and persists at high levels 
of $\alpha A R$ signaling. Data from hypertrophy reversibility experiments with specialized $\alpha A R$ antagonists, a mathematical model, and $\alpha A R$ localization images using BODIPY-prazosin support a reversibility delay mechanism of ligand trapping, where PE is internalized and acts on intracellular $\alpha A R^{\prime}$ s. In addition to our BODIPY-prazosin data in neonatal cardiac myocytes, recent evidence has shown a large population of $\alpha_{1} A R^{\prime} s$ in adult cardiac myocytes [54]. Moreover, expressing $\alpha_{1 a} A R$ 's in R-1F cells were predominately found in intracellular organelles [53] and $40 \%$ of native $\alpha_{1} A R^{\prime} s$ in human smooth muscle cells were intracellular [62]. Prazosin, an $\alpha A R$ antagonist that can act at the sarcolemma and also be transported inside the cell, reversed PE-induced hypertrophy. In contrast, CGP-12177a, a membrane impermeable $\alpha A R$ antagonist, did not reverse PE-induced hypertrophy. The prazosin and CGP-12177a results indicate that the reversibility delay mechanism is primarily at the receptor level and not predominately the result of downstream positive feedback. Additionally, only a mathematical model incorporating intracellular $\alpha A R^{\prime} s$ could reproduce the experimentally observed reversibility delay. Evidence of intracellular $\alpha A R^{\prime} s$ was obtained using BODIPY fluorescently labeled prazosin and competitive binding experiments with PE provide evidence for internalization of PE and prazosin but not CGP-12177a. A similar ligand trapping mechanism may be important in other signaling pathways in the cardiac hypertrophy signaling network. Functional intracellular receptors have been identified for angiotensin II [63], endothelin-A and -B [64], and $\beta$-adrenergic receptors [65] in cardiac myocytes.

$\alpha 1 \mathrm{~A}$ and $\alpha 1 \mathrm{~B}$ receptor subtypes have been shown to colocalize with Gaq and PLC $\beta 1$ at the nuclear membrane in adult mouse cardiac myocytes [22]. Moreover, membrane permeable $\alpha 1 \mathrm{AR}$ antagonist prazosin blocked phosphorylation of ERK in adult mouse cardiac myocytes while membrane impermeable $\alpha 1 A R$ antagonist CGP-12177a did not [22]. To better characterize this mode of signaling, additional studies demonstrating interaction of Gaq with intracellular $\alpha A R$ 's such as with FRET microscopy and experiments showing location of Gaq activity would be valuable. Additionally, 
experiments characterizing the trafficking of vesicular $\alpha A R$ 's and downstream signaling proteins warrant further study.

Hypertrophy reversibility delay and ligand trapping results may have implications for disease progression and treatment for heart failure. Endogenous $\alpha A R$ agonists norepinephrine and epinephrine are released in response to cardiac stress. The reversibility delay result implies that through ligand trapping, hypertrophy signaling can continue for a significant time after transient exposure to circulating catecholamines. Catecholamine uptake data show that cardiac myocytes can internalize substantial levels of norepinephrine within 5 minutes [54]. This memory mechanism, while potentially providing cardioprotection for future insults, could also accelerate the development of hypertrophy and heart failure. Moreover, $\alpha$ AR agonist internalization would complicate therapeutic approaches aimed at blocking this pathway. Through internalization, the cell could circumvent antagonists that act only at the sarcolemma, as seen here with myocytes treated with CGP-12177a. Therefore development of pharmacologic agents that can be readily internalized by the cell or prevent uptake of hypertrophic agonists may be important for successful treatment of cardiac hypertrophy.

Here we used cultured neonatal rat ventricular myocytes in our imaging platform to study cardiac myocyte hypertrophy reversibility. While cultured cells do not fully replicate the complex 3D environment of the heart, they are necessary for the scalable high-throughput experiments needed for systems-wide analysis of hypertrophy signaling networks. Previous studies have demonstrated reversibility in patients after surgical interventions [43-45] and in mice with drug treatments [40-42]. Moreover it was recently shown that hypertrophy and systolic dysfunction induced by constitutively active calcineurin was reversible by solely turning off calcineurin activity, without the requiring any additional treatment [66]. This study has interesting implications concerning the reversibility of hypertrophy when the underlying cause is removed. Furthermore, the reversibility of signaling downstream of calcineurin is consistent with our data that PE-induced hypertrophy is reversible. 
Reversibility of other hypertrophic pathways and specific circumstances that may disrupt this reversibility requires further study.

Comprehensive studies in cultured cells are needed to better understand signaling network organization, cross-talk, and mechanisms of hypertrophy reversibility. This knowledge will help improve pharmacologic and genetic target selection for treatment of cardiac hypertrophy. Moreover, the highthroughput image acquisition and analysis techniques shown here could be extended to be used in highthroughput drug or RNAi screens or with human stem-cell derived cardiac myocytes [67].

In summary, we developed an automated image acquisition and analysis approach for quantifying changes in morphology of individual cardiac myocytes over time. This methodology can accommodate the large number of pharmacologic and genetic perturbations needed to better study the biological circuits controlling cardiac hypertrophy. This approach revealed that PE-induced hypertrophy exhibits a concentration-dependent reversibility delay that can be explained by intracellular ligand trapping. 


\section{Chapter 3}

\section{Network reconstruction and systems analysis of cardiac myocyte hypertrophy signaling.}

Reprinted from: Network reconstruction and systems analysis of cardiac myocyte hypertrophy signaling, Vol. 287, No. 50, December 2012, Pages 42259-68, with permission from the American Society for Biochemistry and Molecular Biology. 


\subsection{Introduction}

Cardiac hypertrophy develops in response to biochemical and mechanical stresses, increasing patient risk of heart failure and malignant arrhythmia [12]. The cardiac hypertrophy response is managed by a dense web of signaling pathways with many species influencing cardiac myocyte growth [13]. The complexity of this network has hindered the development of successful therapeutic strategies [3] and indicates the need for integrative systems approaches which can provide a global view of functional relationships in the network [34].

Computational models have been used to increase understanding of the role of signaling components and topology on cardiac physiology such as myocyte contractility [68], arrhythmia [69], and hypertrophy [70], [71]. While these models have been used successfully to address focused questions about individual pathways, more global network reconstructions are needed to understand differential regulation of hypertrophy and crosstalk between these pathways. Large-scale integrative models have been successful in other systems such as the prediction of optimal evolution [72] and drug targets [73] in metabolic networks and prediction of the global transcriptional response to genetic and environmental perturbations [74].

Here, we developed a computational model of the hypertrophy signaling network by integrating many established pathways implicated in cardiac myocyte growth. We used the recently described normalized-Hill modeling framework [75] with default parameters, which allowed us to build a more complete network despite limited available quantitative biochemical data. We used this model to determine how the components and network topology of hypertrophy signaling lead to differential regulation of transcription factors, gene expression, and myocyte size. Model predictions were validated using published and new experimental data testing the effects of receptor activation on transcription factors and myocyte phenotypic outputs. Using this model we analyzed network motifs, dynamics, and modules to increase understanding of network organization and performed sensitivity analysis to 
identify global functional relationships in the network. Key findings include identification of Ras as an influential network hub, enrichment of network motifs causing crosstalk, and many nodes that influence myocyte hypertrophy, but only a few nodes with large positive or negative effects on cell growth.

\subsection{Experimental Procedures}

\subsubsection{Modeling Approach}

Network reconstruction of cardiac hypertrophy signaling focused on the most established pathways leading from receptor inputs to transcription factor activities, gene expression, and myocyte size. Each reaction was substantiated with at least two citations from the literature, with a preference for data from neonatal rat ventricular myocytes. The overall cardiac hypertrophy signaling network model contains 106 species and 193 reactions (Figure 3.1). The model contains 17 receptor inputs (tumor necrosis factor $\alpha$, TNF $\alpha$; isoproterenol, ISO; norepinephrine, NE; phenylephrine, PE; endothelin-

1, ET1; insulin-like growth factor , IGF1; epidermal growth factor, EGF; angiotensin II, AngII; neuregulin 1, NRG1; transforming growth factor $\beta$, TGF $\beta$; interleukin 6, IL6; fibroblast growth factor, FGF;

cardiotrophin 1, CT1; leukemia inhibitory factor, LIF; stretch, brain naturetic peptide, BNP; atrial naturetic peptide, ANP) and 7 phenotypic outputs (cell area and expression of six genes: sarcoplasmic

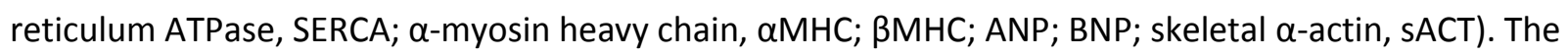
size of the model allowed for investigation of differential regulation of hypertrophy and crosstalk between pathways. 


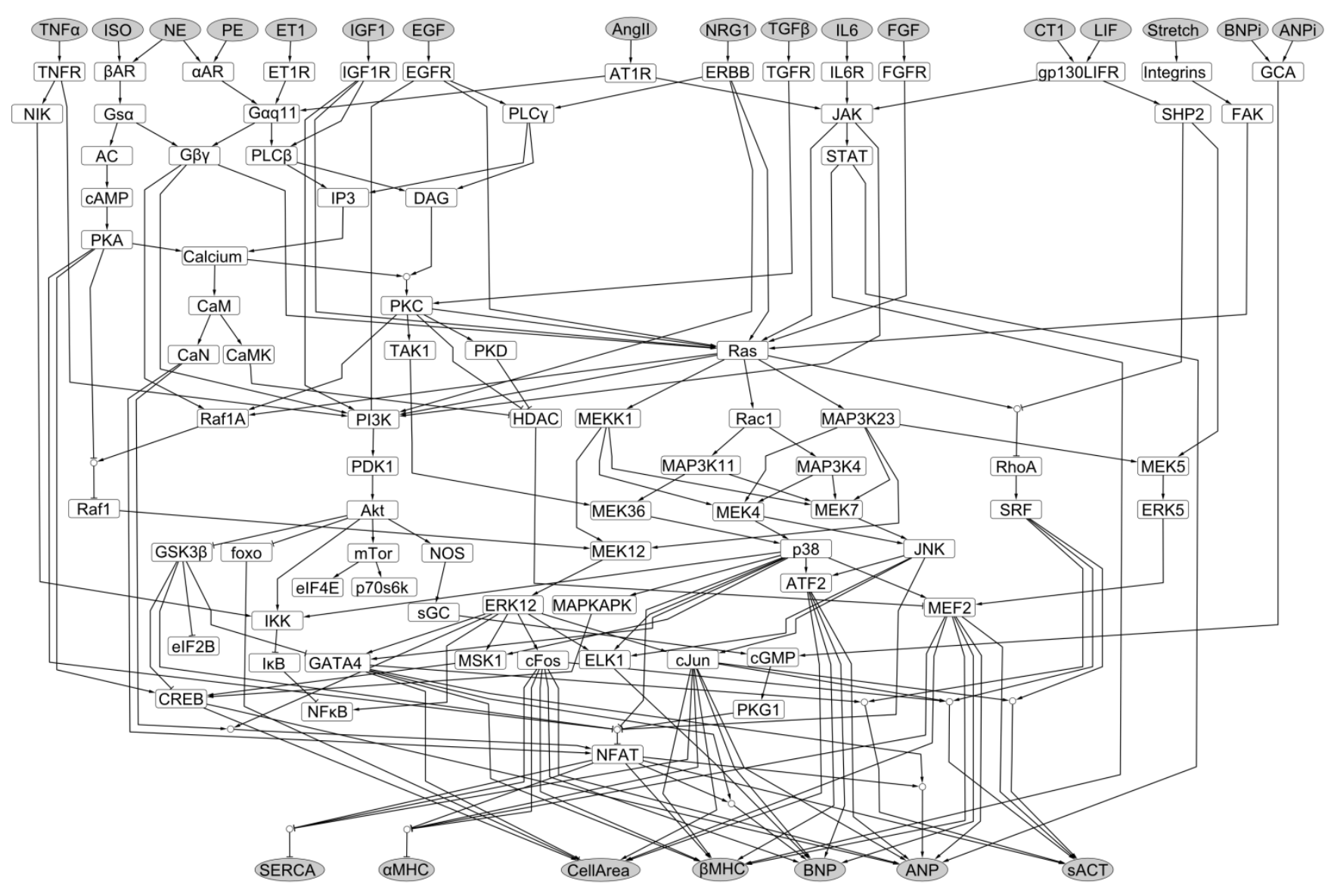

Figure 3.1: Schematic of the cardiac hypertrophy signaling network model. The model consists of 106 species and 193 reactions. Model inputs and outputs are shown in gray. This model was implemented using the normalized-Hill differential equation modeling approach (12) using 132 literature citations focused on neonatal rat ventricular myocytes (see Appendix C). 
Species dynamics were predicted by converting the network to mathematical equations using the recently described normalized-Hill differential equation approach [75]. Briefly, this modeling approach uses logic-based differential equations, representing activation or inhibition reactions using normalized Hill functions together with logical AND and OR gates to compute crosstalk. Each species has a corresponding differential equation, which is computed in units of fractional activation so that protein abundance parameters are not required. For example, the joint activation of protein kinase C (PKC) by calcium (Ca) and diacylglycerol (DAG) is represented by the following equations:

1) $\frac{d P K C}{d t}=\frac{1}{\tau}\left(\frac{B \cdot C a^{n}}{K^{n}+C a^{n}} \cdot \frac{B \cdot D A G^{n}}{K^{n}+D A G^{n}} \cdot P K C_{\max }-P K C\right)$

2) $B=\frac{E C_{50}^{n}-1}{2 E C_{50}^{n}-1}$

3) $K=(B-1)^{\frac{1}{n}}$

An example of an OR gate is seen in the activation of JNK by MEK4 or MEK7:

4) $\frac{d J N K}{d t}=\frac{1}{\tau}\left(\left(\frac{B \cdot M E K 4^{n}}{K^{n}+M E K 4^{n}}+\frac{B \cdot M E K 7^{n}}{K^{n}+M E K 7^{n}}-\left(\frac{B \cdot M E K 4^{n}}{K^{n}+M E K 4^{n}} \cdot \frac{B \cdot M E K 7^{n}}{K^{n}+M E K 7^{n}}\right)\right) \cdot J N K_{\max }-J N K\right)$

Default parameters (specified in Appendix C: weight $=1, n=1.4$, tau $=1$ and $E C 50=0.5$ ) were used for all reactions based on a prior normalized-Hill model of the cardiac $\beta$-adrenergic pathway [75]. MATLAB code for the 106 differential equations was generated automatically from the table in Appendix C using Netflux (freely available at http://code.google. com/p/netflux). Initial values for each species were 0 or 1 and are listed in Appendix C. The normalized-Hill framework allows predictions of network dynamics and is compatible with many analyses from the field of nonlinear dynamics while requiring minimal knowledge of biochemical parameters. Notably, the kinetics of this model can be refined as experimental data become available [75]. 


\subsubsection{Analysis of Network Topology}

The hypertrophy signaling network was exported from Netflux into Cytoscape [76] for topological analysis. The Network Analyzer [77] plug-in was used to calculate topological properties of the network such as the mean number of neighbors and the characteristic path length from input to output (Table 3.1). The NetMatch [78] plug-in was used to identify enriched network motifs. Motifs are biologically significant network structures that form the building blocks of a complex system, often facilitating regulation, stability, and cross-talk [79]. To identify statistically enriched network motifs, the hypertrophy signaling network was compared to a set of ten randomized models with a scale-free degree distribution. These randomized models were created using the RandomNetworks plug-in (http://sites.google.com/site/randomnetworkplugin). Comparisons were performed for five different motifs: three-node feed-forward loops, bi-parallels, four-node feed-forward loops, bi-fans, and threenode feedback loops. The $\mathrm{z}$-score for each comparison was calculated using the equation: $\mathrm{Z}=\left(\mathrm{N}_{\text {hyp }}{ }^{-}\right.$ $\left.N_{\text {rand }}\right) / S_{\text {rand }} . N_{\text {hyp }}$ is the number of a particular motif in the hypertrophy network, $N_{\text {rand }}$ is the average number of that motif in the randomized scale free networks, and $\mathrm{S}_{\text {rand }}$ is the standard deviation.

\subsubsection{Sensitivity Analysis and Identification of Network Modules}

A sensitivity analysis was performed by simulating individual knockdowns for each of the 106 species in the network and then measuring the activation of all species in the network at steady state. Knockdowns were generated by setting the maximal activity for a given species to zero (e.g. $P K C_{\max }=0$ ). All 17 input reactions were set to a weight of 0.072 such that cell area was close to 0.5 , maximizing the information that could be obtained from sensitivity analysis. This is based on the experimental observation that unstimulated neonatal myocytes are roughly half the size of myocytes stimulated with a strong hypertrophic agonist [80]. Results were combined as a $106 \times 106$ sensitivity matrix defined as: $S_{i j}$ $=\Delta \mathrm{Y}_{\mathrm{i}} / \Delta \mathrm{P}_{\mathrm{j}}$, where $\mathrm{S}_{\mathrm{ij}}$ is the sensitivity of species " i" to knockdown of species " $\mathrm{j}$ ", $\Delta \mathrm{Y}_{\mathrm{i}}$ is the change in 
steady-state output of species " $\mathrm{i}$ " (control-knockdown), and $\Delta \mathrm{P}_{\mathrm{j}}=1$ when species " $\mathrm{j}$ " is being knocked down.

In order to determine which species in the network have similar functions, k-means clustering was applied after thresholding the sensitivity matrix. This threshold was applied at a sensitivity level of 0.001 , where sensitivities above the threshold were set to 1 and all values below the negative of the threshold were set to -1 . All other values were set to zero. In Cytoscape, species could be collapsed into their modules using the plug-in Metanodes (http://www.cgl.ucsf.edu /cytoscape/metanodes /metanodes.html), providing a map of how the modules were interconnected with each other.

\subsubsection{Cell Culture and Microscopy}

Cardiac myocytes were harvested from 1-2 day old Sprague Dawley rats after decapitation using the Neomyts isolation kit (Cellutron, Baltimore, MD). All procedures were performed in accordance with the Guide for the Care and Use of Laboratory Animals published by the US National Institutes of Health and approved by the University of Virginia Institutional Animal Care and Use Committee. Myocytes were cultured in plating media (Dulbecco Modified Eagle Media, 17\% M199, 10\% Horse Serum, 5\% Fetal Bovine Serum, $100 \mathrm{U} / \mathrm{mL}$ penicillin, and $50 \mathrm{mg} / \mathrm{mL}$ streptomycin) on Cellbind treated 96 -well plates (Corning, Corning, NY) at a density of 100,000 cells/well. Two days after isolation, myocytes were transfected with GFP driven under a cardiac myocyte specific troponin T promoter $^{1}$ using Lipofectamine 2000 (Invitrogen, Carlsbad, California; transfection efficiency: 10-15\%).

Two days after transfection, myocytes were imaged using an Olympus IX81 inverted microscope with 10X UPlanSApo 0.40 NA objective, Orca-AG CCD camera (Hamamatsu, Bridgewater, NJ), automated stage (Prior Scientific, Rockland, MA), and IPLab software (Scanalytics, Fairfax, VA). Images were acquired with 120 ms exposure time using a 480/40-nm excitation filter and 535/50-nm emission filter (Chroma filters; Optical Insights, Santa Fe, NM). After imaging, cells were rinsed and transferred to a solution of $100 \mu \mathrm{mol} / \mathrm{L}$ Ras inhibitor (FPT Inhibitor III), $100 \mu \mathrm{mol} / \mathrm{L} \mathrm{JNK}$ inhibitor (SP600125), $10 \mu \mathrm{mol} / \mathrm{L}$ 
p38 inhibitor (SB203580), or $10 \mu \mathrm{mol} / \mathrm{L}$ of MEK1/2 (U0126) inhibitor (Calbiochem, La Jolla, CA) in serumfree media (Dulbecco Modified Eagle Media, 19\% M199, 1\% ITSS, 100U/mL penicillin, and $50 \mathrm{mg} / \mathrm{mL}$ streptomycin). After 1 hour, the myocytes were transferred to a solution of $10 \mu \mathrm{mol} / \mathrm{L} P \mathrm{P}$, an $\alpha-$ adrenergic receptor ( $\alpha A R$ ) agonist, with a given inhibitor. Follow-up images were recorded after 24 hours. In each well of interest in the 96-well plate, a 5x5 grid of images was collected automatically using custom image acquisition scripts [39]. Changes in myocyte area were evaluated using automated custom MATLAB algorithms [39]. Differences in fold change in cell area were tested for statistical significance using Kruskall-Wallis non-parametric one-way analysis of variance followed by a Dunn's multiple comparisons post-test test.

\subsection{Results}

\subsubsection{Topology of the Hypertrophy Signaling Network}

The construction of a cardiac hypertrophy signaling network model creates an opportunity to examine how global network properties influence the development of hypertrophy. Previous studies have primarily examined effects of a single species or pathway using biochemically detailed mass action or Michaelis kinetics. Here, we used the recently described normalized-Hill modeling framework [75] with default parameters, allowing us to model a more integrative signaling network despite limited available quantitative biochemical characterization at this scale. Comprehensive sensitivity analysis comparing a previously built mass action model of $\beta$-adrenergic signaling with detailed parameters with a normalized-Hill model with default parameters showed high agreement, with a Pearson correlation

coefficient of 0.754 [75]. Key reasons for the high agreement between these two modeling approaches are the normalization of equations in terms of fractional activation and the separation of steady state (e.g. reaction weights) and kinetic (time constants) parameters.

Topological properties of the overall network are summarized in Table 3.1. Species with a small number of neighbors and species with many neighbors are overrepresented compared to a network 


\begin{tabular}{|c|c|}
\hline Number of Species & 106 \\
\hline Number of Reactions & 193 \\
\hline Avg. Number of Neighbors & 3.508 \\
\hline Characteristic path length & 4.936 \\
\hline Hubs (8+ neighbors) & $\begin{array}{l}\text { Ras, p38, cJun, NFAT, MEF2, ERK12, } \\
\text { GATA4, PI3K }\end{array}$ \\
\hline Inputs & $\begin{array}{l}\text { AngII, ANP, BNP, CT1, EGF, ET1, FGF, } \\
\text { IGF1, IL6, ISO, LIF, NE, NRG1, PE, } \\
\text { Stretch, TGF } \beta \text {, TNF } \alpha\end{array}$ \\
\hline Transcription factors & $\begin{array}{l}\text { ATF2, cFos, cJun, CREB, foxo, } \\
\text { GATA4, MEF2, NFAT, SRF }\end{array}$ \\
\hline Outputs & $\begin{array}{l}\alpha \mathrm{MHC}, \mathrm{ANP}, \beta \mathrm{MHC}, \mathrm{BNP}, \text { CellArea, } \\
\text { SACT, SERCA }\end{array}$ \\
\hline
\end{tabular}

Table 3.1: Topological properties of the cardiac hypertrophy signaling network. The number of neighbors is equivalent to the total number of reactions that go in or out of a given species.

Characteristic path length is the average number of nodes between two species in the network. 


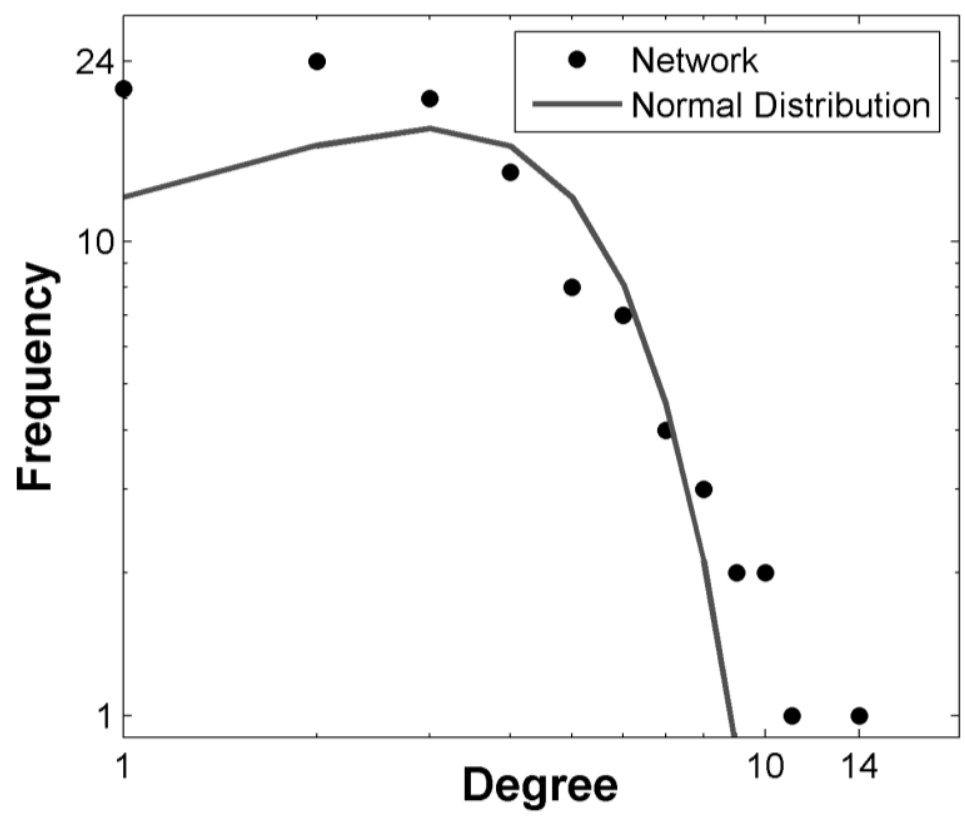

Figure 3.2: Hypertrophy signaling network degree distribution. Log-log plot of degree (number of neighbors) distribution of nodes in the hypertrophy network compared to a normal distribution with the same median and standard deviation as the hypertrophy network. The hypertrophy network degree distribution fails the D'Agostino and Pearson omnibus normality test. In the hypertrophy signaling network, species with low degree are more abundant than species with high degree and enriched compared to the normal distribution. Additionally, the network has more species with much higher degree ( $\geq 10$, hubs) than would be expected in a network with a normal degree distribution. 
with normally distributed linkages (Figure 3.2). Eight nodes with an especially high number of neighbors ( $\geq 8)$, "hubs [81]," were identified (Table 3.1). Since hubs participate in a large number of reactions in the network, they may be influential across several parallel pathways in the hypertrophy network.

Network motif analysis revealed the presence of feed-forward loop, bi-fan, and bi-parallel motifs in the hypertrophy network. While a high level of cross-talk was expected based on the network diagram, the specific types of interaction motifs present and their significance compared to other networks of the same size was not known. The structure of each identified motif, two examples from the hypertrophy network, and quantitative comparisons to the randomized networks with the same number of species and interactions are shown in Table 3.2. Feed-forward loops indicate cross-talk between species in parallel pathways and influence reaction speed. Feed-forward loops in the hypertrophy network were primarily longer path four-node loops instead of three-node loops. Threenode feed-forward loops were present, but underrepresented in the hypertrophy network compared to randomized scale-free networks. Bi-parallel motifs were overrepresented in the network while other types of four-node feed-forward loops were absent. Bi-fan motifs were highly overrepresented in the hypertrophy network compared to the randomized scale-free networks, indicating a high level of crosstalk between pathways in the hypertrophy signaling network.

\subsubsection{Simulation and Experimental Validation of Network Dynamics}

The normalized-Hill differential equation framework of the hypertrophy signaling network model enables network-wide prediction of signaling dynamics. Figure 3.3 shows an example simulation of the response to a transient exposure to phenylephrine (PE) followed by TNFa. Different patterns of activation between the two hypertrophic agonists can be clearly observed as well as groups of network species with similar patterns of activation. For example, members of the Ras/MAPK pathway are activated with PE and not TNF $\alpha$ while PI3K is activated by both agonists. Moreover, similar patterns of activation can be seen in small GTPases Rac1, Raf1A, Ras, and RhoA as well as members of the MAPK 


Feed-

Table 3.2: Enriched network motifs in the hypertrophy signaling network. Motif analysis revealed feedforward loop, bi-fan, and bi-parallel network motifs in the cardiac hypertrophy network. The structure of each motif is shown along with two specific examples present in the network. The prevalence of these motifs was compared to the average of ten randomized scale-free models. $\mathrm{N}_{\text {hyp }}$ is the number of motifs in the hypertrophy network and $\mathrm{N}_{\text {rand }}$ is the average number of motifs in the randomized networks. The z-score comparing prevalence in the hypertrophy and the randomized models is also shown. Bi-fan motifs were largely prevalent in this network while feed-forward loops were underrepresented compared to the randomized models. 


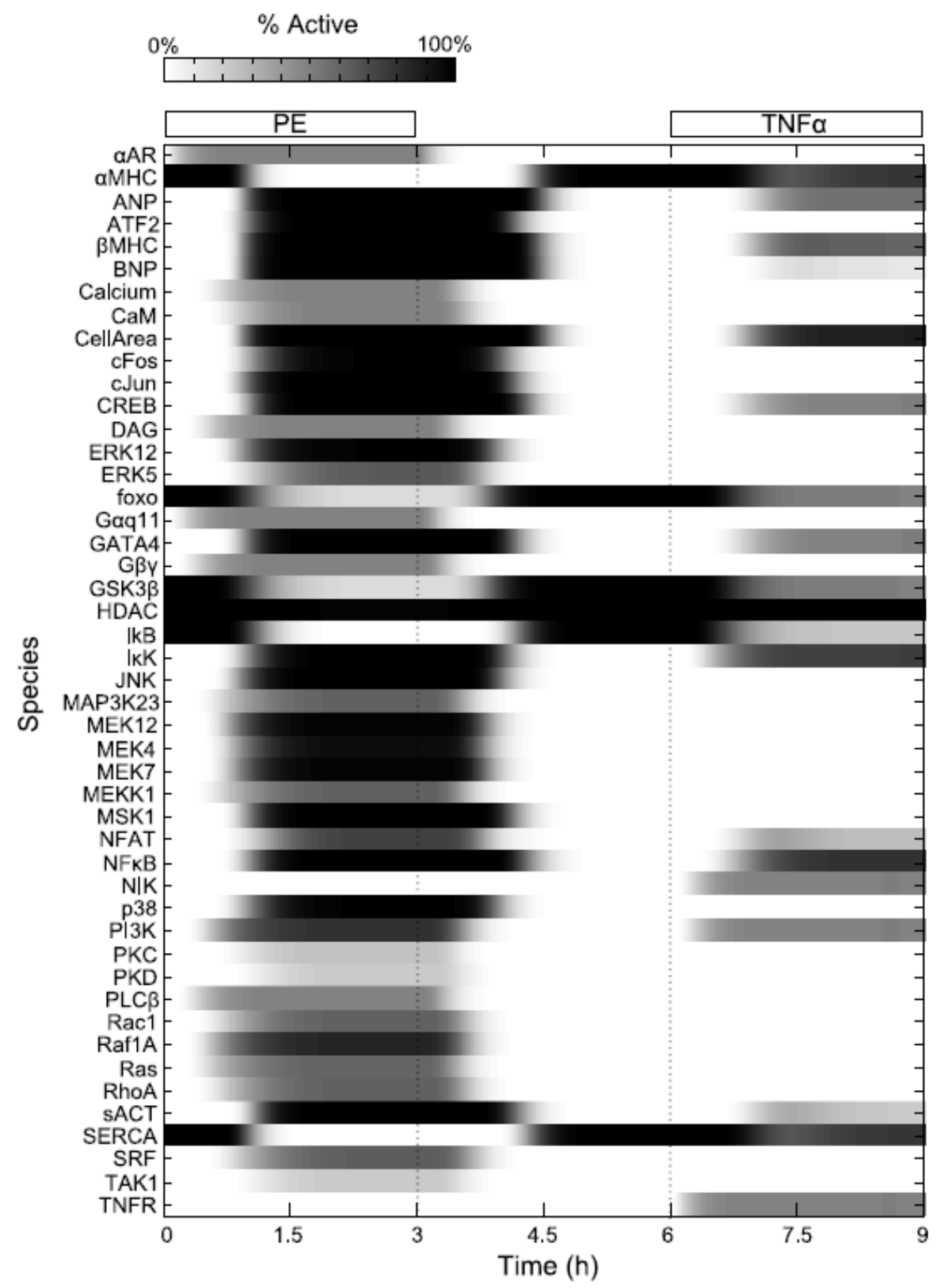

Figure 3.3: Predicting global dynamics of the hypertrophy signaling network. Predicted activation time course for a subset of species (47/106) in the cardiac hypertrophy signaling network model comparing perturbations by phenylephrine (PE) and tumor necrosis factor alpha (TNF $\alpha$ ) to show how different ligands induce different dynamic network responses. PE was removed to restore the original system steady state before adding TNF $\alpha$. 
pathway MEK12, MEK4, and MEK7. While the current model uses default time constants for all reactions, these parameters can be readily revised as kinetic experimental data become available [75].

The model was validated using independent data from the experimental literature testing the effects of each model input on the phenotypic outputs (see Figure 3.4). Experimental sources are listed in Appendix D. Model data was binned into the categories of positive, negative, and no effect at steady state due to limits in the resolution of the available experimental data. The hypertrophy model was able to correctly predict 109 of 114 (96\%) qualitative input-output relationships observed experimentally in neonatal ventricular myocytes. A notable exception is the model prediction that IGF1 increased ANP gene expression, which is the opposite of experimental observations [82]. Experimental data was found for only $48 \%$ of these input-output relationships, indicating substantial gaps in the literature. Model discrepancies with experimental data such as this will help inform model revision and experimental design.

\subsubsection{Sensitivity Analysis of Myocyte Hypertrophy}

Sensitivity analysis provides a global view of quantitative functional relationships between every species in the hypertrophy signaling network. Figure 3.5 shows the sensitivity analysis for a subset of the network, while the full 106 x 106 species sensitivity matrix for the entire network is shown in Figure 3.6. Each column of the matrix shows the change in activity of each species in the model when a given species is knocked down. Therefore the diagonal of the matrix represents self-activation, which varies between species as a result of differences in baseline activity.

Sensitivity analysis revealed quantitative relationships in the hypertrophy signaling network that would not be apparent from observation of the network topology alone. By examining a particular row of the sensitivity matrix one can see what species most strongly influence a particular species. For example, ANP gene expression (row 2 ) is more strongly influenced by Ras than JAK and more strongly influenced by JAK than JNK, even though each of these species is in the same pathway. 

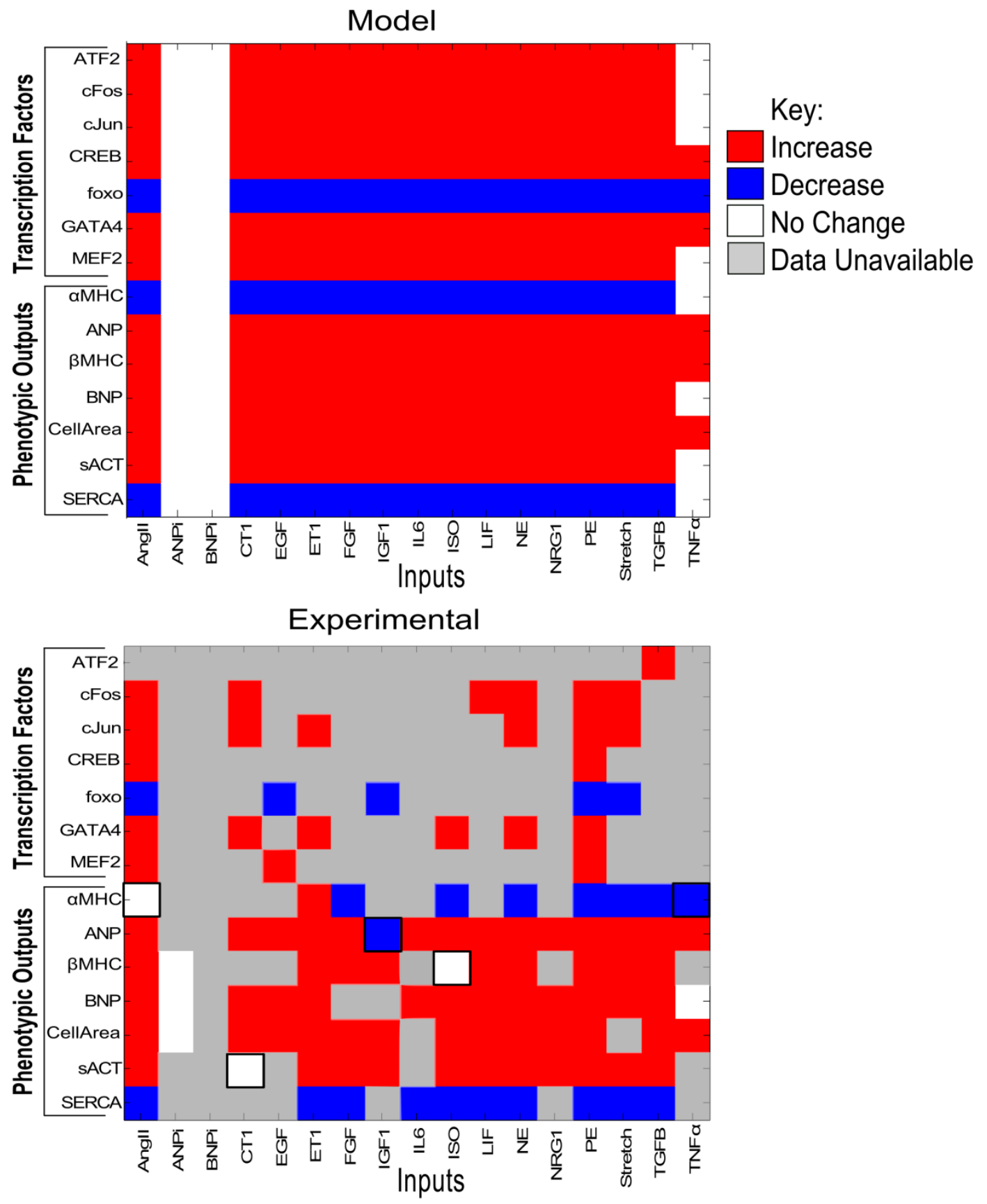

Figure 3.4: Experimental validation of model predictions from receptor activation to transcription factors and phenotypic outputs. Red indicates increase, blue indicates decrease, white indicates no change, and grey indicates an absence of available data. The cardiac hypertrophy model accurately predicted the large majority of qualitative input-output relationships of the hypertrophy network (109 of 114 relationships, sources provided in Supplemental Table 2). Discrepancies between the model and experimental results are boxed. 


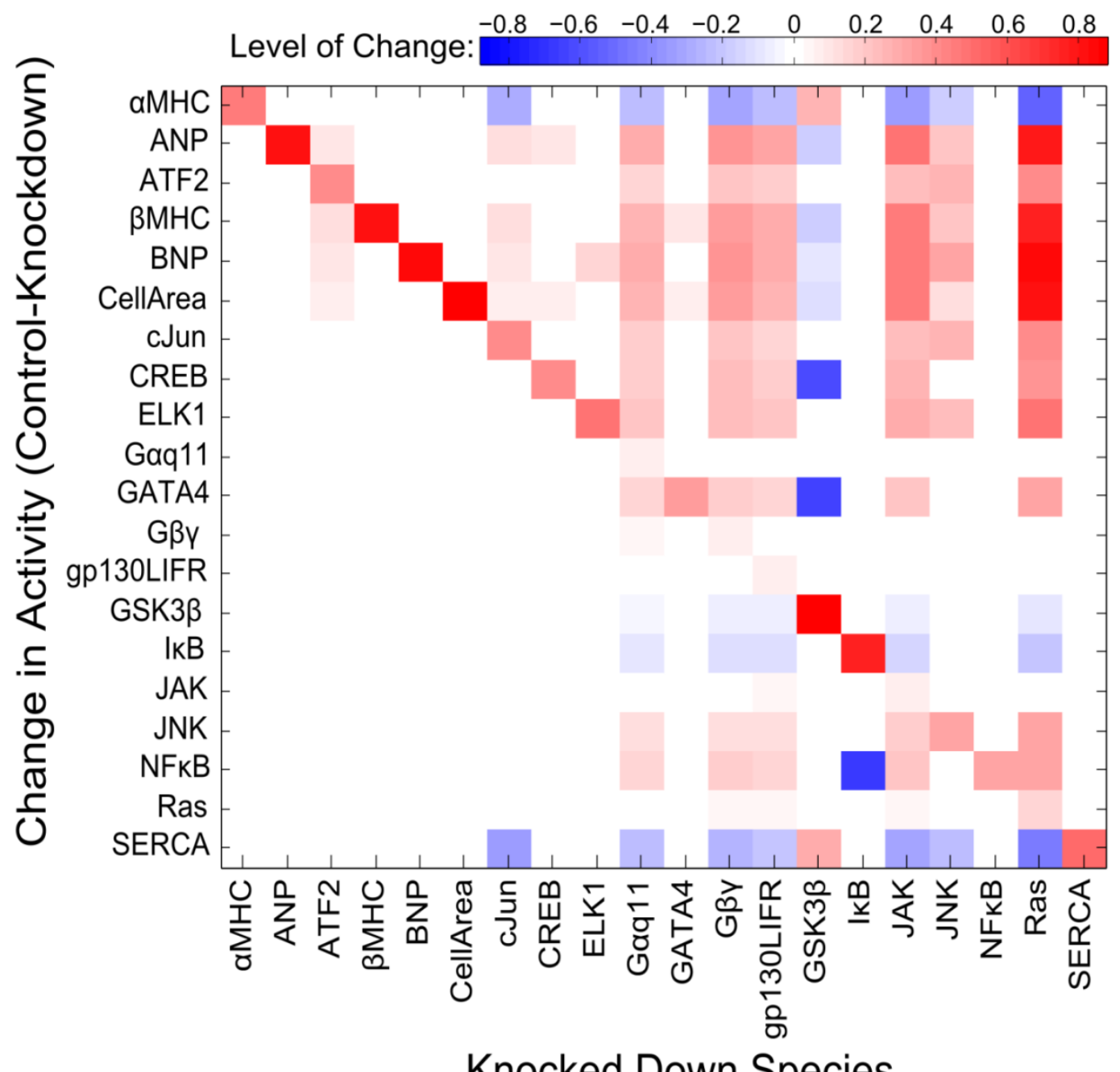

Figure 3.5: Sensitivity analysis reveals global functional relationships of the hypertrophy signaling network. A subset of the full sensitivity matrix for the cardiac hypertrophy signaling network model is shown. Each column of the sensitivity matrix represents a computational experiment in which the labeled species was knocked down and then the activity of each species in the model was measured at steady state. Sensitivity values indicate change in steady state output (control - knockdown). Therefore red signifies that the species in the column activates the species in the row, while blue signifies inhibition and white signifies no change. 


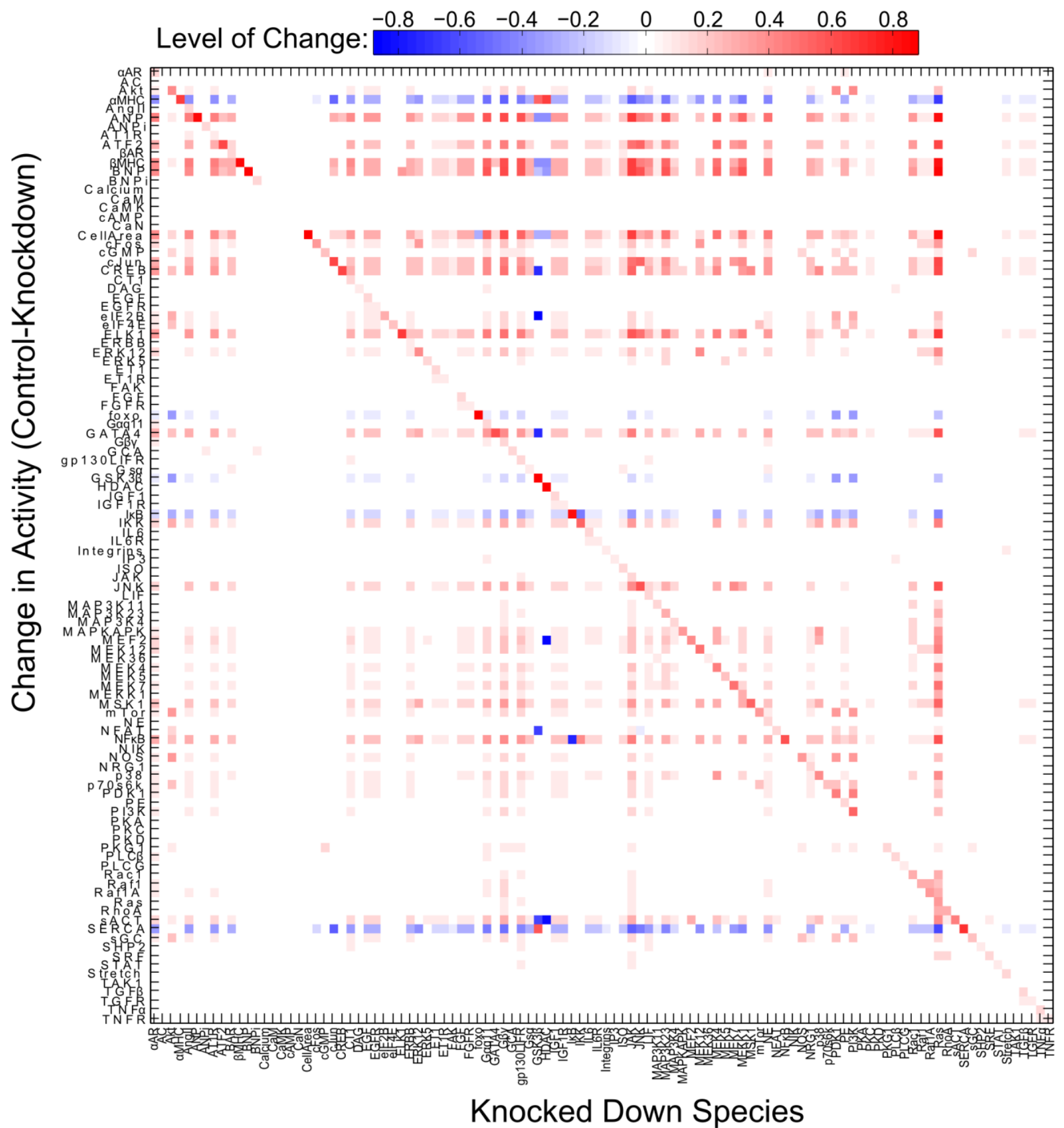

Figure 3.6: Sensitivity matrix for the entire hypertrophy signaling network. Each column of the sensitivity matrix represents a computational experiment in which the labeled species was knocked down and then the activation of all species in the model was measured at steady state. Sensitivity values were computed as described in Methods. Red indicates that the species in the column activates the species in the row, while blue indicates inhibition and white indicates no change. 
By examining the columns of the sensitivity matrix the global influences of every species in the network can be compared. For example, GSK3 $\beta$ (column 14) has a greater inhibitory role in the network compared to other species and may represent a potential therapeutic target for overexpression/activation. Figure 3.5 also shows that Ras has a greater relative influence on species in the network compared to other species such as GBy. Moreover, species further upstream in the network such as Gaq11 and gp130LIFR tend to affect more species in the network than species further downstream such as ATF2 and ELK1. Interestingly, while Ras affects a large number of species in the network, Ras is not significantly affected by many species in the network. Since Ras has 14 direct connections to other species in the network, including 8 activating species, Ras can retain a high level of activation despite reduced levels of a single upstream species. While we hypothesized strong correlations between the number of connections with other species in the network and sensitivity and influence [81], [83], sensitivity analysis revealed only mode rate correlations between local connectivity and global network influence and sensitivity in the hypertrophy network (Figure 3.7). Notable exceptions to the expected connectivity/function correlation included a substantial influence of ET1 despite direct links only to its receptor ET1R, and a high sensitivity of NFKB despite being directly regulated only by IKB and ERK1/2.

The row of the sensitivity matrix corresponding to Cell Area (row 6) highlights species with major influences on cell growth. Ras, JAK, GßY, Gaq11, and gp130LIFR have the highest influences on Cell Area in the network. The single species with the greatest influence on cell area, Ras, is also a hub, with 14 connections to other species in the network. GSK3 $\beta$ negatively influences Cell Area. A network view of the relative influence on Cell Area of each species is shown in Figure 3.8. This diagram shows highly influential species at various locations in the network, not just near the outputs. With these results we rank ordered the species in the network based on their degree of influence on Cell Area from least to greatest (Figure 3.9A). This information will be useful for prioritizing future experiments and 


\begin{tabular}{|l|r|r|r|}
\cline { 2 - 4 } \multicolumn{1}{c|}{} & In Degree & \multicolumn{1}{c|}{ Out Degree } & \multicolumn{1}{c|}{ Degree } \\
\hline Influence & -0.34 & 0.29 & -0.13 \\
\hline Sensitivity & 0.62 & -0.04 & 0.50 \\
\hline
\end{tabular}

B
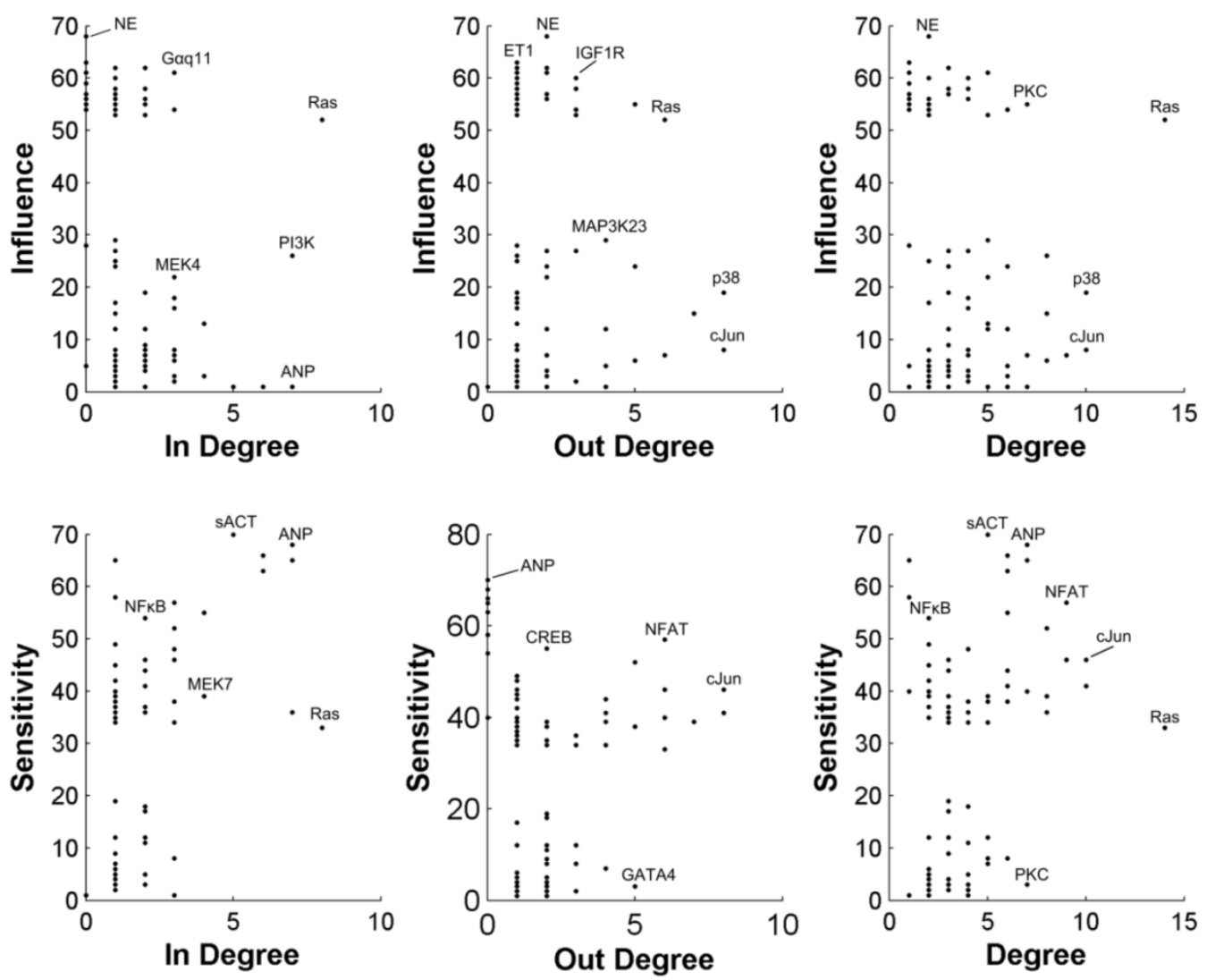

Figure 3.7: Correlations between local connectivity, influence, and sensitivity. A) Degree is the sum of in-degree (the number of reactions entering a node) and out-degree (the number of reactions exiting a node). The number of species a given node affects (Influence, column of sensitivity matrix) and the number of species that affect a given node (Sensitivity, row of sensitivity matrix) were calculated for each species using the network sensitivity matrix with a threshold of 0.001 . Spearman correlation coefficients were calculated to quantify the relationship between local connectivity and global influence and sensitivity. While correlations between out-degree and influence and in-degree and sensitivity were expected to be high, results show only a moderate correlation. B) Scatter plots depicting the relationships between influence and sensitivity with in-degree, out-degree, and degree. Results show high variation of sensitivity and influence for a given value of degree in the hypertrophy signaling network. 


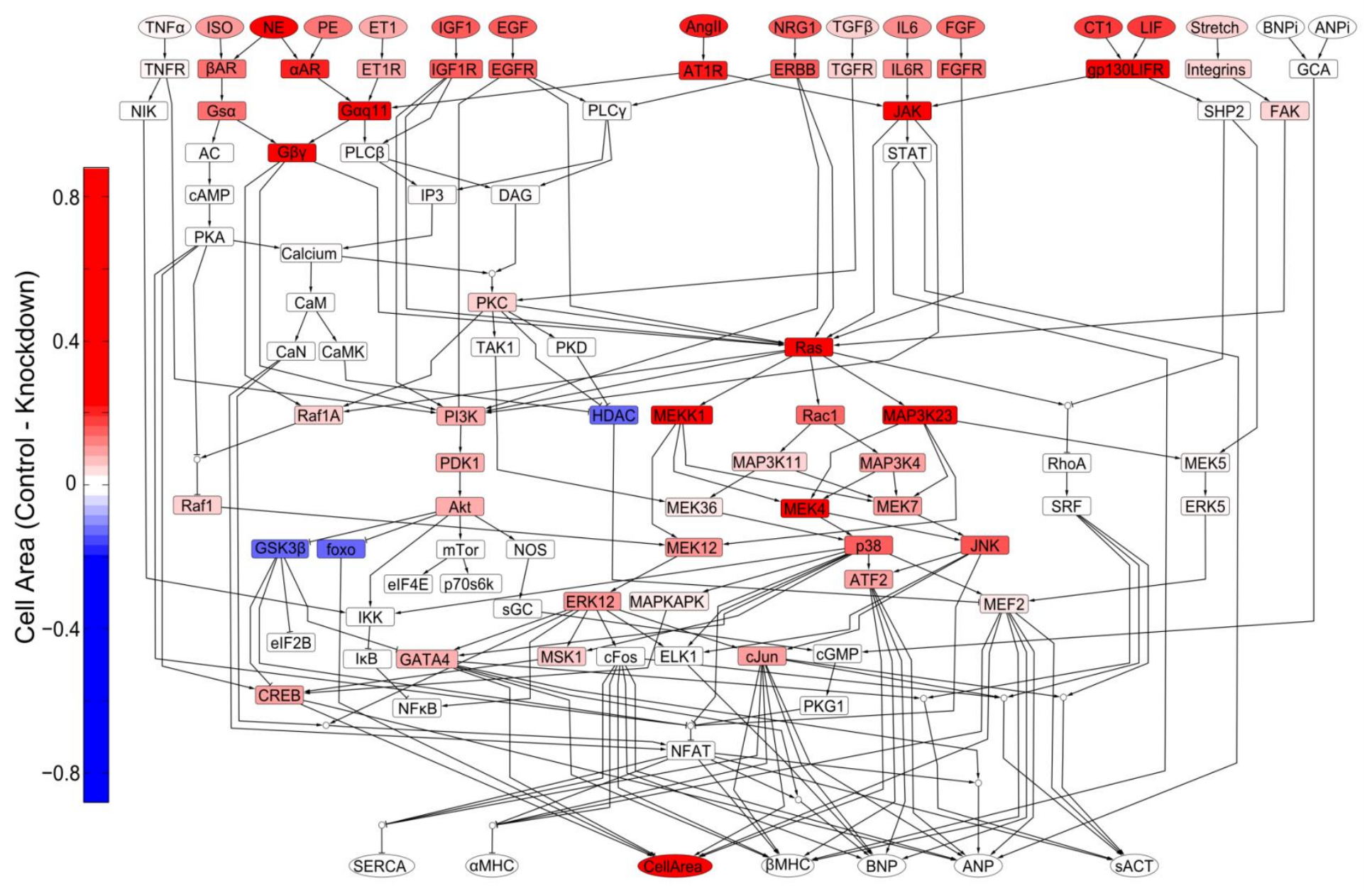

Figure 3.8: Schematic of cardiac hypertrophy signaling network labeled with degree of influence on cell area. Sensitivity coefficients for cell area (Difference in cell area, control-knockdown) for each species in the cardiac hypertrophy signaling network model mapped onto the network diagram to depict the degree of influence on cell area for each species. Red nodes indicate the species has a positive effect on cell area while blue nodes indicate a negative effect. Overall the strongest positive regulators of cell area were located at the receptor level or involved in MAPK signaling. 


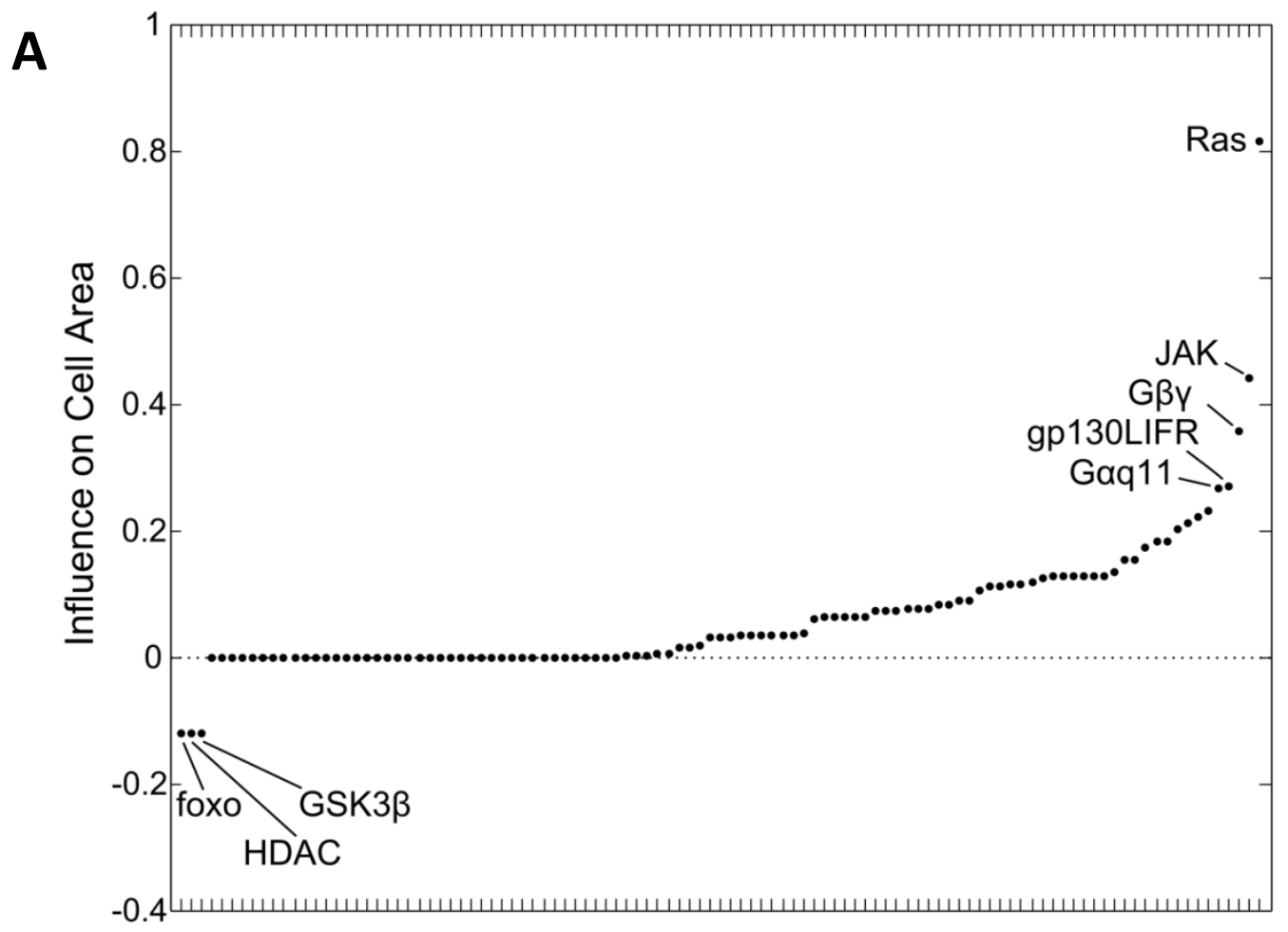

\section{Species}

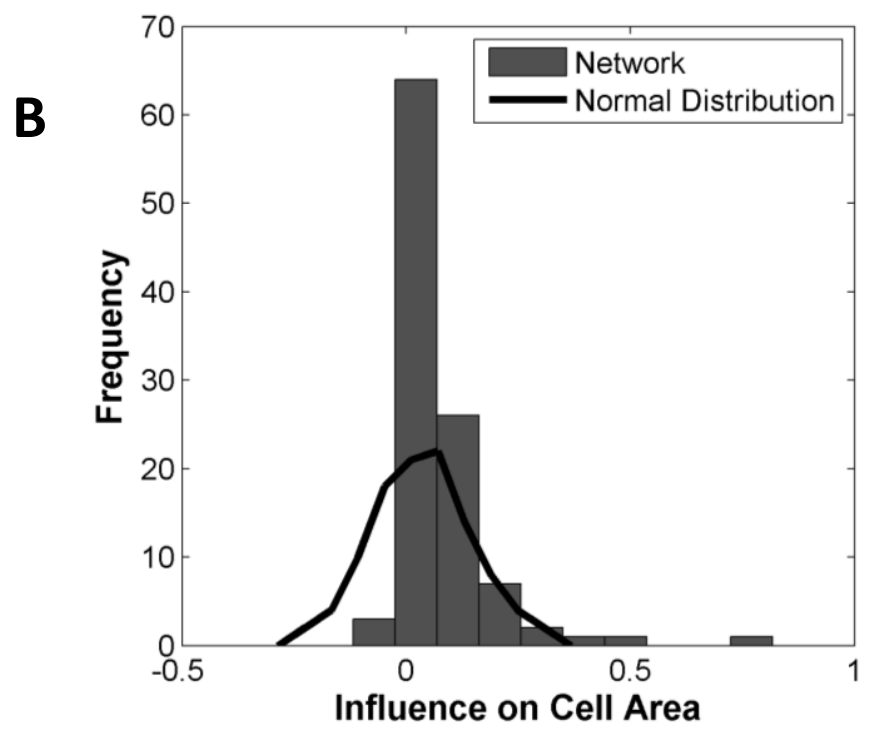

Figure 3.9: Influence of individual species on cell area. A) Each species in the hypertrophy signaling network model was rank ordered based on its level of influence on cell area using the sensitivity coefficients for cell area from Figure S2. Most of the species in the model positively influenced cell area with Ras, JAK, G $\beta \gamma$, and Gaq11 having the largest effects. Three species inhibited cell area, HDAC, foxo, and GSK3 $\beta$. B) Histogram of distribution of level of influence on cell area of species in the hypertrophy network compared to a normal distribution with the same median and standard deviation as the network. Compared to a normal distribution, species with modest positive influences are highly overrepresented and species with negative influences on cell area are underrepresented. Moreover, network species Ras, JAK, and G $\beta \gamma$ had much higher influences on cell area than would be expected in a network with a normal distribution of influences on cell area. The network degree distribution failed the D'Agostino and Pearson omnibus normality test. 

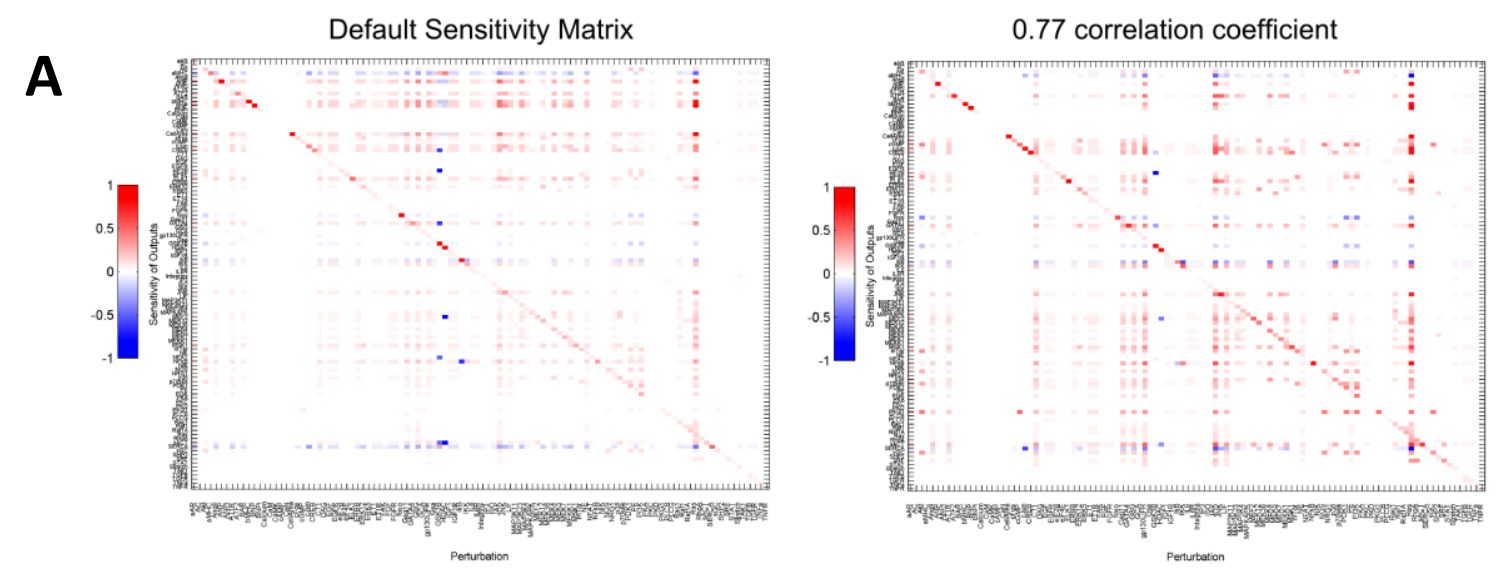

B
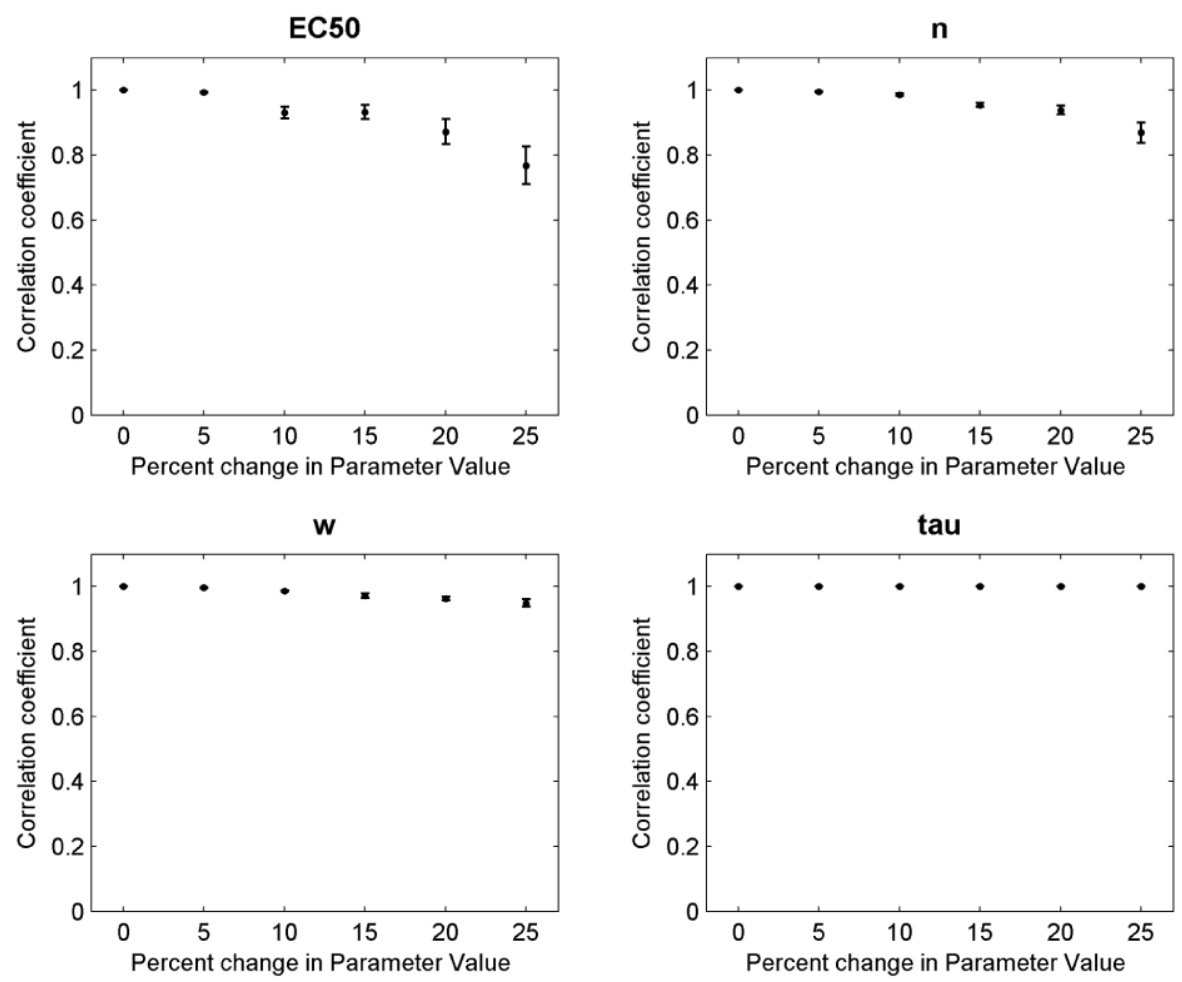

Figure 3.10: Evaluation of model sensitivity to choice of default parameters: A) Sensitivity matrix for default parameter choice (left) compared to a sensitivity matrix with a Pearson correlation coefficient of 0.77 (right). The sensitivity matrix on the right was produced by allowing values for EC50 to fall along a uniform probability distribution $+/-25 \%$ of the default value of 0.5 . Qualitatively the two sensitivity matrices are very similar, with most of the differences being in the relative magnitude of the values. B) We adjusted each parameter value using a uniform probability distribution $+/-0$ to $25 \%$ of the default parameter value and calculated the Pearson correlation coefficient of the resulting sensitivity matrix compared to the default sensitivity matrix. Simulations were repeated 5 times per condition. Correlation coefficients remained high despite large distributions of parameter values. The model was most sensitive to the selection of EC50. These results provide confidence that model results are robust to changes in default parameter values. 
potential drug targets. Compared to a normal distribution, species with low positive influences are overrepresented, species with negative influences are underrepresented, and Ras, JAK, and G $\beta \gamma$ have much higher influences on cell area than expected (Figure 3.9B). To ensure that the model was not highly sensitive to our choice of default parameter values, we examined the correlation coefficients between the model with default parameter and models with choices of EC50, $n$, weight, and tau based on a uniform probability distribution within a given range (Figure 3.10).

\subsubsection{Sensitivity Analysis Reveals Modular Regulation of Hypertrophy}

We hypothesized that there may be an underlying structure to the sensitivity matrix that would help elucidate functional organization of the hypertrophy signaling network. Rather than forming modules based on network structure, correlations in the rows of the sensitivity matrix were automatically sorted by k-means clustering into twelve functional modules in the hypertrophy network (Figure 3.11). A network schematic labeling the module for each species is shown in Figure 3.12. Of these, the largest module (Receptors) contained every input and corresponding receptor except for $\beta$ adrenergic receptor ( $\beta A R$ ). This module also included connected species PKC, TAK1, PKD, and HDAC. This large module occurred because each of these species is located "upstream" in the hypertrophy network and therefore affected by a limited number of other species. The MAPK species were partitioned into two groups, one consisting of species closely neighboring Ras and one centering on p38. Species in the downstream module centering on p38 are affected by more species than the upstream module that included Ras. Species that are predominately inhibited by other species in the network (SERCA, aMHC, IKB, foxo, GSK3 3 ) were also grouped together. NFAT's unique mixture of inhibition and activation by other species in the network resulted in it being grouped by itself.

The MAPK and PI3K modules contain major network hubs, suggesting a role of these modules as network integration points. For example, the PI3K module is centered on a species with high in-degree (PI3K) and one with high out-degree (AKT). Other modules represent discernible, linear pathways such 


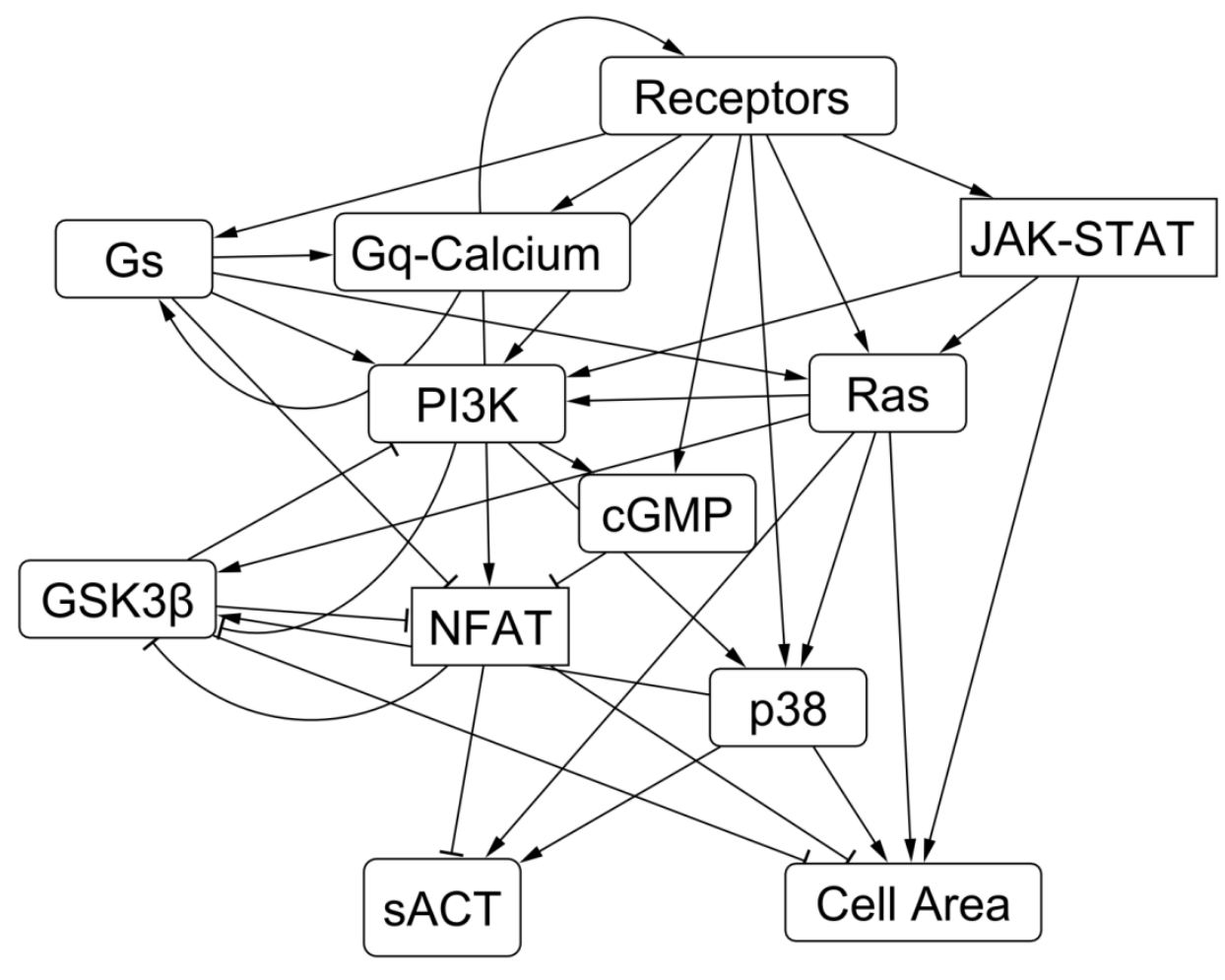

Figure 3.11: Modular organization of the hypertrophy signaling network revealed by sensitivity analysis. k-means cluster analysis of the sensitivity matrix of the cardiac hypertrophy model revealed twelve major functional signaling modules. Labeled connections between modules represent interactions between species in different modules. 


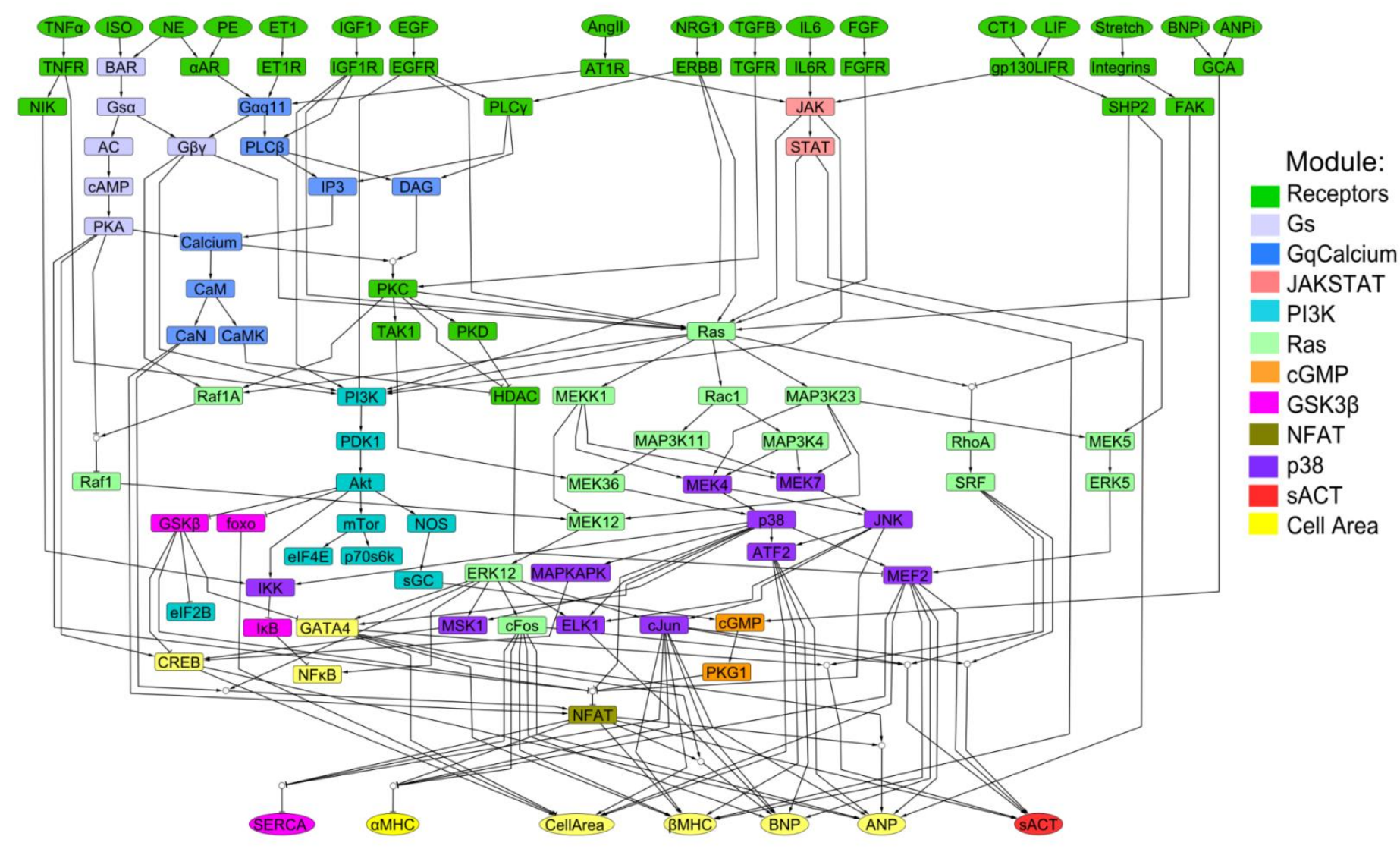

Figure 3.12: Schematic of cardiac hypertrophy signaling network, labeling module for each species. kmeans clustering of the sensitivity matrix revealed twelve functional modules in the hypertrophy network. The color of each species indicates which module the species was assigned to during clustering. Module names represent influential species in a particular module. 
as $\beta A R, G q / C a l c i u m$, and JAK/STAT modules. Network outputs were organized in three different modules suggesting differences in regulation and indicating the network's ability to generate different hypertrophic phenotypic outputs in different signaling states.

The network was sorted into twelve modules because this group number resulted in moderate sized modules. Expanding the number of groups beyond twelve resulted in more small groups with $\leq 4$ members. For example, with fifteen modules Gq-Calcium signaling split into three modules and SERCA and $\alpha \mathrm{MHC}$ split from the GSK3 $\beta$ module. Reducing the module number below twelve resulted in more modules joining the large receptors module. For example, reducing the number of groups to ten modules resulted in Gs signaling grouping together with the large receptors module and SACT joining the Cell Area module.

Connections between modules are labeled (Figure 3.11) in order to characterize interactions between network components. Modules were on average connected to 5.17 other modules in the network. Motif analysis revealed 22 feed-forward loop, 29 bi-parallel, and 21 bi-fan motifs between network modules. These results indicate a high level of cross-talk between modules and suggest that studying the interactions between signaling pathways will be important in understanding cellular regulation of cardiac hypertrophy.

\subsubsection{Experimental Validation of Distributed Processing by the Ras/MAPK Pathway}

The hypertrophy signaling model includes eight hubs (Table 3.1), which are species with at least eight connections to other species in the network. The sensitivity analysis revealed that the network hub Ras has the greatest influence on cell area in the network (Figure 3.5-6). No other network hubs make up the list of the five most influential network species (Supplemental Figure 3.9A). The second most influential species, JAK, only had six direct connections to other species (Figure 3.1). Ras has 14 connections to other species in the network, six of which are downstream. Through distributed processing out of Ras, the network could continue to generate large increases in cell area if any single 


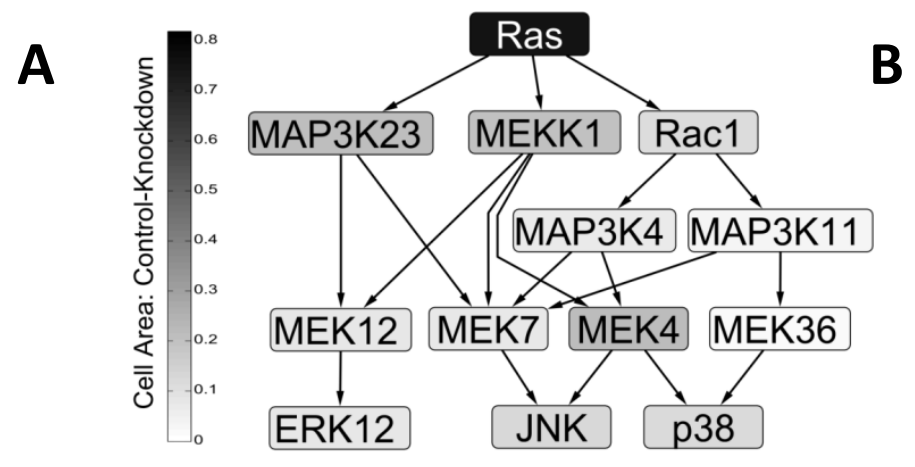

Model

C

Experiment (24 hours)

D

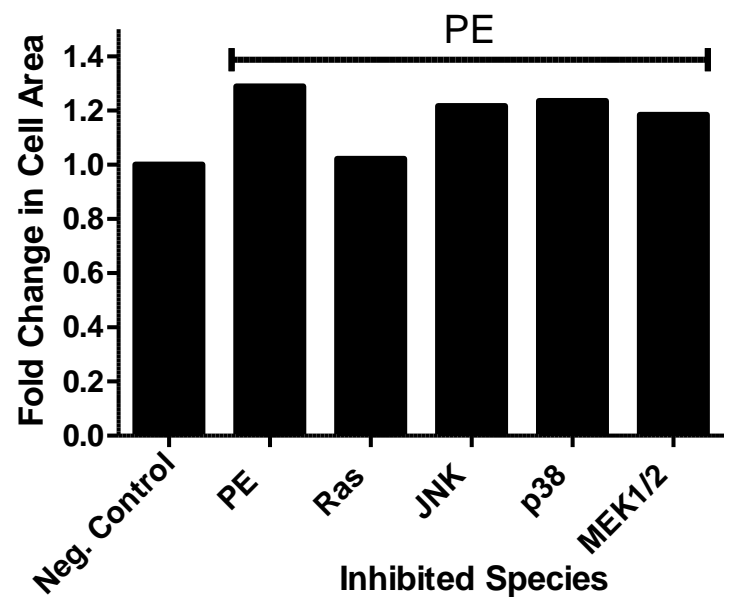

Experiment (48 hours)
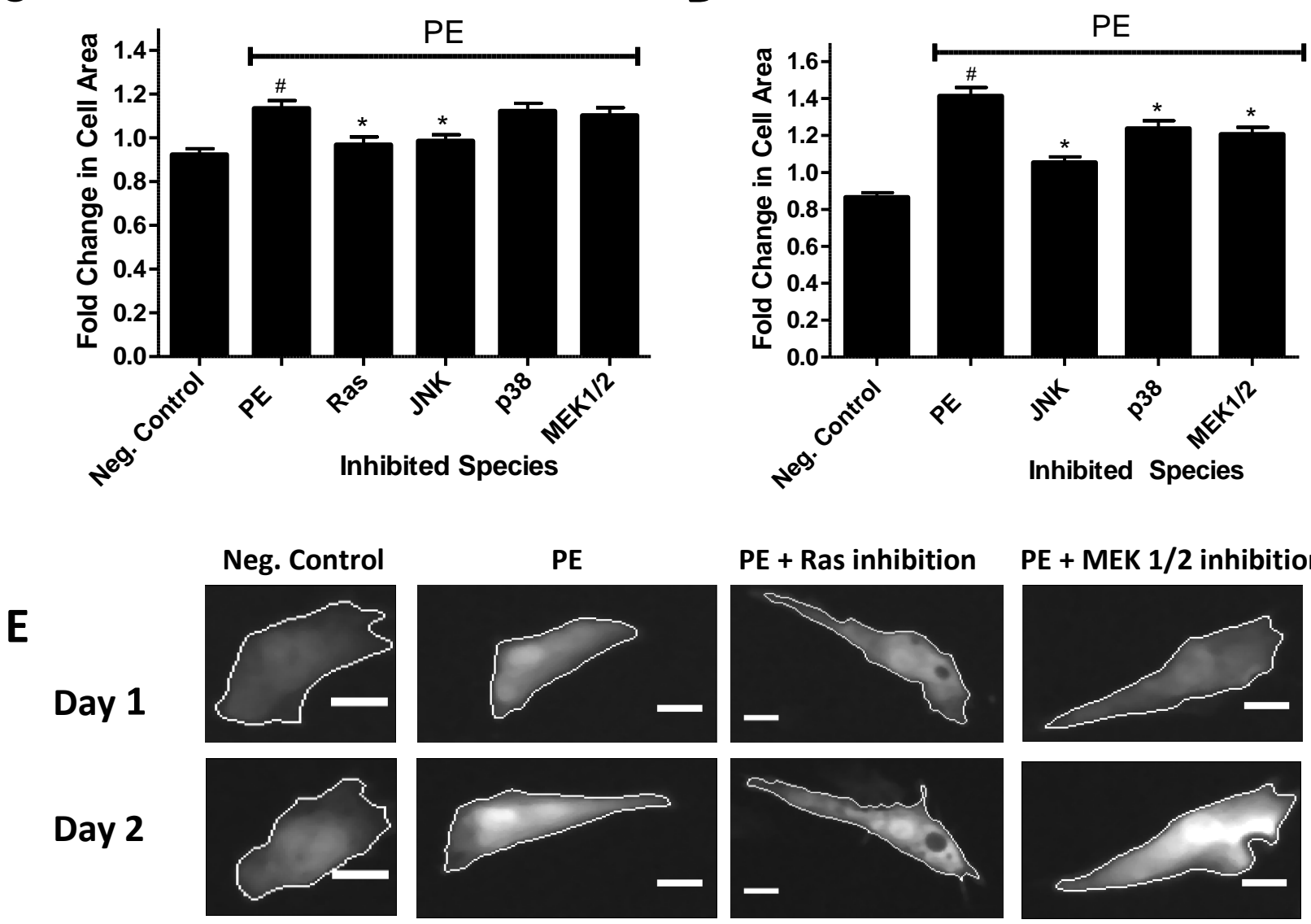

PE + Ras inhibition

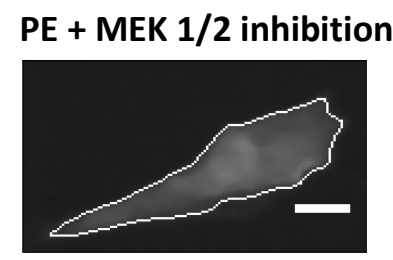

Fold change

in cell area

0.85

1.16

1.04

1.13

Figure 3.13: Model predictions and experimental validation of distributed processing by the

Ras/MAPK pathway. A) Schematic of signaling downstream of Ras labeled with predicted differences in cell area between control and the knockdown of each species in the pathway. Darker species labels indicate a larger influence on cell area. The model predicts that inhibiting Ras, which has the largest influence on cell area, would have a much larger effect on PE-induced cardiac hypertrophy than knocking down any individual downstream species. B) Model predicted fold change in cell area for PE- 
induced hypertrophy with Ras, JNK, MEK1/2, or p38 inhibition. The PE input reaction was set to a weight of 0.25 and all other inputs were turned off. C) Median ( $\sim 500$ cells per condition) fold change in cell area of cultured neonatal rat ventricular myocytes stimulated for 24 hours with PE with an inhibitor for either

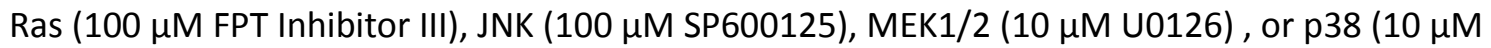
SB203580). Error bars are +/- SE. As predicted, inhibiting Ras almost entirely prevented PE-induced hypertrophy. Differences in fold change in cell area were tested for statistical significance using KruskallWallis non-parametric one-way analysis of variance followed by a Dunn's multiple comparisons posttest. \# $\mathrm{P}<.05$, comparison with negative control (PE vs neg. control). ${ }^{*} \mathrm{P}<.05$, comparison with positive control (Ras, JNK, MEK1/2, and p38 inhibition vs. PE). D) Median ( 700 cells per condition) fold change in cell area of cultured neonatal rat ventricular myocytes stimulated for 48 hours with PE with an inhibitor for JNK (10 $\mu$ M SP600125), MEK1/2 (100 nM PD325901), or p38 (10 $\mu$ M SB203580) for 48 hours. Ras inhibition combined with PE resulted in cell death at 48 hours, so we could not include data from this condition. E) Representative images of segmented cardiac myocytes after 24 hours of treatment with labeled fold change in cell area (Scale bar: $10 \mu \mathrm{m}$ ). PE-induced hypertrophy is attenuated by Rasinhibition more than MEK1/2 inhibition. 
pathway out of Ras was blocked. The model therefore predicts that blocking Ras would have a larger effect on cardiac hypertrophy than inhibiting any other downstream species alone (Figure 3.13A,B).

To test this model prediction we stimulated cardiac myocytes with the $\alpha A R$ agonist PE. The magnitude of PE-induced hypertrophy was compared to myocytes stimulated with both PE and an inhibitor for either Ras, JNK, MEK1/2, or p38. As predicted by the model, Ras inhibition almost entirely prevented PE-induced hypertrophy, giving further evidence for the role of Ras as a highly influential network hub in hypertrophy signaling (Figure 3.13C,E). MEK1/2 and p38 inhibition had smaller effects on cell area (Figure 3.13C-E). JNK inhibition, however, had a greater impact on cell growth than expected based on model predictions (Figure 3.13C). This finding may suggest a larger role in cell growth than can be predicted by the current model or reflect limitations in specificity of the JNK inhibitor [84]. Therefore the relative contribution to hypertrophy between MEK1/2, p38, and JNK requires further study. Data from experiments taken out to 48 hours reveal that $p 38$ inhibition and MEK1/2 inhibition did have significant effects on hypertrophy, but less so than JNK inhibition (Figure 3.13D). Ras inhibition combined with PE resulted in cell death at 48 hours, so we could not include data from this condition. Since sustained Ras inhibition with PE led to cell death, Ras may be important in adaptation to stress for cardiac myocytes. Ras inhibition with FPT Inhibitor III has been shown to increase apoptosis [85], [86]. Inhibitor treatment without PE did not cause significant $(\mathrm{P}<.05$, Kruskal Wallis test followed by Dunn's multiple comparison's post-test) differences in fold change in cell area compared to negative control at 24 or 48 hours (Figure 3.14). 

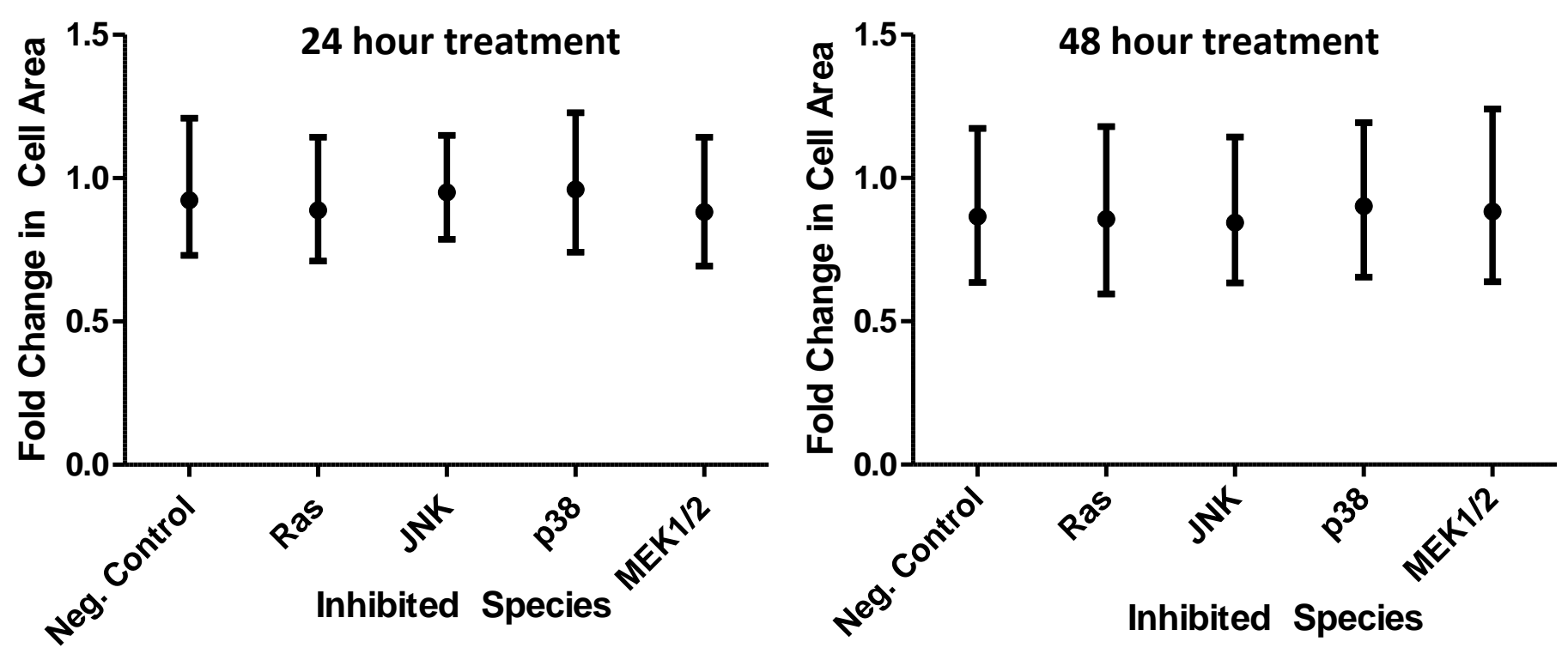

Figure 3.14: Fold change in cell area for myocytes treated with inhibitors but without PE. To ensure that the inhibitors used were not causing substantial effects on cell health or hypertrophy, we treated myocytes with the same concentrations of inhibitors used to collect the data displayed in Figure 6C-D without PE. Graph displays median and interquartile range of fold change in cell area ( $N \sim 500$ cells per condition). Myocytes in A) were treated for 24 hours with an inhibitor for either Ras (100 $\mu$ M FPT

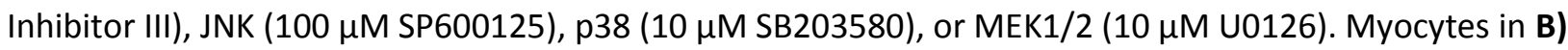
were stimulated for 48 hours with an inhibitor for Ras (100 $\mu$ M FPT Inhibitor III), JNK (10 $\mu$ M SP600125), p38 (10 $\mu$ M SB203580), or MEK1/2 (100 nM PD325901). The concentrations of inhibitors used did not cause significant differences in fold change in cell area compared to control (serum-free media only). Differences in fold change in cell area were tested for statistical significance using Kruskall-Wallis nonparametric one-way analysis of variance followed by a Dunn's multiple comparisons post-test test. $P<.05$ was considered significant. 


\subsection{Discussion}

While previous experiments related to hypertrophy have focused primarily on small portions of the overall network, integrated computational models provide an opportunity to analyze how multiple pathways interact to affect cardiac hypertrophy. Here, we developed a computational model of the hypertrophy signaling network with 106 species and 193 reactions by integrating 14 established pathways regulating cardiac myocyte growth. Other models of hypertrophy have been constructed to increase understanding of individual pathways in the network. A previous model of the $\mathrm{IP}_{3}$-calcineurin pathway used sensitivity analysis to show that differences in receptor kinetics and density explained the differences in $\mathrm{IP}_{3}$ transients induced by endothelin-1 and angiotensin II [71]. A different model of the calcineurin pathway was used to elucidate MCIP's role in a negative feedback loop in calcineurin/NFAT signaling that requires a large threshold of NFAT to induce MCIP expression [70]. These studies successfully used systems analysis tools to gain new insights about the composition of signaling pathways related to hypertrophy. We built a more comprehensive model of the larger network in order to study how network organization and interactions between network components affect the differential regulation of transcription factors, gene expression, and myocyte size.

Network motif analysis revealed an enrichment of bi-fan and bi-parallel motifs, network building blocks that could accelerate the development of hypertrophy and impede its reversal. Bi-fan motifs indicate a high level of cross-talk and further motivate the need for integrative systems techniques that examine the larger network in order to better understand hypertrophic signaling. Bi-parallel motifs are a type of four-node feed-forward loop. Feed-forward loops have been shown in mathematical models and Escherichia coli transcriptional regulation to accelerate signaling and increase robustness of the network by delaying output reversal when the upstream signal is removed [79]. This facilitates output stability with the transient loss of an input [87]. These properties can be further tuned by adjusting parameters in the network such as thresholds and reaction rates [88]. As experimental data becomes available, 
reaction parameters of individual motifs can be refined in the hypertrophy signaling network model to study specific feed-forwards regulating signaling speed and robustness.

Sensitivity analysis was used to inform clustering of the network into modules. Modularity enhances robustness of a particular module's function and distinct connections between modules enable development of various cell functions [89]. Modules in the hypertrophy network were found to be highly interconnected with an enrichment of feed-forward, bi-fan, and bi-parallel motifs. More experimental data is needed to fully characterize the unique role of each of these modules in hypertrophy.

Sensitivity analysis identified species with the greatest effects on cell growth. GSK3 $\beta$ was predicted to negatively regulate cell area which is consistent with the finding that GSK3 $\beta$ overexpression attenuated the hypertrophy response due to calcineurin, $\beta$-adrenergic signaling, and pressure overload [90] and partially reversed hypertrophy due to pressure overload [91]. Upstream nodes were in general more influential on the overall network than downstream nodes, which can also be seen in the sensitivity analysis for the $\beta$-adrenergic signaling network (12). Ras, a network hub, had the greatest effect on cell area and greater magnitude effects on more species than any other species in the network. The high influence of Ras is consistent with the correlation of network degree and lethality of single gene mutations [81] and the stability of scale free networks to random node failures and not to attacks on hubs [83].

We tested our model prediction that attack of the hub Ras would have a greater effect on cell area than inhibition of less connected downstream nodes by comparing the level of PE-induced hypertrophy with Ras, JNK, MEK1/2, or p38 inhibitors. As predicted, the Ras inhibitor had the greatest effect on PE-induced hypertrophy. Our experimental results demonstrating reduction in PE induced hypertrophy with inhibition of Ras, JNK, p38, and MEK $1 / 2$ are consistent with data from cultured neonatal myocytes [92], [93]. Increased cell size was seen in neonatal rat cardiac myocytes with 
microinjection of an activated Ras mutant [94], overactivated JNK by constitutively active MKK7 [95], overactivated p38 by constitutively active MKK6 [93], and expression of constitutively active MEK1 [96]. While in vitro experiments show pro-hypertrophic effects for JNK, MEK1/2, and p38, in vivo experiments have generated more conflicting results [97]. Additional studies are needed to conclusively show the relative contributions of these ligands to various cardiac hypertrophy phenotypes in myocytes.

Reactions in this hypertrophy model were selected based on published data with a preference for experiments using cultured neonatal rat ventricular myocytes. While neonatal cells are perhaps less physiologically relevant to cardiac hypertrophy compared to adult myocyte or in vivo systems, more complete experimental data on hypertrophy signaling is available from neonatal myocytes. This allowed us to build a more comprehensive signaling network model than would have otherwise been possible. The use of default parameters with equal weighting of all reactions was necessary for this initial model since many of these parameter values are unknown. Weighting and parameter values can be refined as experimental data become available. Similarly, this extensible platform allows newly identified reactions to be added as they are discovered. Experiment-model discrepancies highlight areas where additional signaling proteins or reactions may be needed to mechanistically explain experimental observations. The model may therefore be a useful tool for experimental design.

In summary, we developed an integrative model of the hypertrophy signaling network in neonatal ventricular myocytes. Motif analysis revealed a high level of cross-talk and four-node feedforward motifs in the network, and sensitivity analysis identified the most influential species in hypertrophy and modular organization of the network. We demonstrated the utility of this approach in studying the functional effects of multi-scale signaling network organization on cardiac myocyte hypertrophy. 


\section{Chapter 4}

\section{Phenotypic screen identifies distinct signatures of cardiac myocyte hypertrophy}

Reprinted from: Phenotypic screen quantifying differential regulation of cardiac myocyte hypertrophy identifies CITED4 regulation of myocyte elongation., Vol. 72, July 2014, Pages 74-84, with permission from Elsevier. 


\subsection{Introduction}

Cardiac hypertrophy develops with increased biochemical and mechanical stresses on the heart [12] and is a major predictor of heart failure and sudden cardiac death [98-100]. Specific features of cardiac hypertrophic remodeling depend on the type of cardiac stress [101], [102]. Physiological stresses such as exercise lead to hypertrophy without cardiac dysfunction, but pathological stresses such as high blood pressure and myocardial infarction lead to hypertrophy with increased fibrosis, cell death, and cardiac dysfunction [17]. Moreover, myocytes grow distinctly in response to different mechanical stimuli. Pressure overload induces concentric hypertrophy, characterized by thickening of the heart wall, and volume overload of the heart induces eccentric hypertrophy, characterized by myocyte elongation and dilation of the heart wall [103]. Since eccentric hypertrophy is a greater risk to patients than concentric hypertrophy [31], increased knowledge of the unique signaling pathways controlling myocyte shape will be important in improving therapies for heart failure. Previous work suggests that myocyte size is regulated by common signaling pathways and myocyte shape is regulated by distinct signaling pathways [37], [104]. But little is known about the specific signaling pathways that induce distinct characteristics of hypertrophy [18], [105].

Many signaling pathways and genes manage the hypertrophic response [13]. While many of the components of this network have been identified, the distinct contributions to different features of hypertrophy such as shape, fibrosis, and cell death between pathways is not well understood [106]. Furthermore, the pathways governing hypertrophy are highly connected with much cross-talk between pathways [107]. It is unclear how all of the parts of such an interconnected network function together as a coordinated system that can induce distinct, context-dependent hypertrophy features. Commonly measured markers of hypertrophy such as cell size and fetal gene expression are not markedly differentially regulated between receptor pathways in the signaling network [107]. 
Here, we test the hypothesis that features such as myocyte shape, fibrosis, cell death, and inflammation may better differentiate hypertrophic signaling pathways. We quantified differential regulation of 5 shape features using high-throughput myocyte imaging and transcript levels of 12 genes induced by 15 predominant hypertrophic agonists. These genes have previously been associated with phenotypes such as fibrosis, cell death, contractility, proliferation, angiogenesis, inflammation, and the fetal cardiac gene program, providing a phenotypic signature for each agonist. We clustered pathway inputs and outputs to identify a network map linking input modules to output modules. Among these, we found strong correlations between Bax and CTGF mRNA abundance in response to Angll and between myocyte elongation and CITED4 mRNA abundance in response to Nrg1. Follow-up experiments validated these correlations, revealing regulation of pro-apoptotic Bax by fibrosis marker CTGF and negative regulation of myocyte elongation by CITED4 gene expression.

\subsection{Methods}

\subsubsection{Cell culture and microscopy}

Cardiac myocytes were harvested from 1 to 2 day old Sprague Dawley rats using the Neomyts isolation kit (Cellutron, Baltimore, MD). Myocytes were cultured in plating media (Dulbecco's modified Eagle media, 17\% M199, 10\% horse serum, 5\% fetal bovine serum, $100 \mathrm{U} / \mathrm{mL}$ penicillin, and $50 \mathrm{mg} / \mathrm{mL}$ : streptomycin) at a density of 100,000 cells per well of a $96-$ well plate coated with SureCoat (a combination of collagen and laminin, Cellutron). All procedures were performed in accordance with the Guide for the Care and Use of Laboratory Animals published by the US National Institutes of Health and approved by the University of Virginia Institutional Animal Care and Use Committee. Two days after isolation, myocytes were transfected with GFP under a cardiac myocyte specific troponin T promoter[47] using Lipofectamine 2000 (Invitrogen, Carlsbad, California; transfection efficiency: 1015\%). Two days after transfection, myocytes were imaged using automated image acquisition scripts, 
which collect a $5 \times 5$ grid of images in each well of interest in the 96-well plate (Figure 4.1)[39]. Images were collected using an Olympus IX81 inverted microscope with 10X UPlanSApo 0.40 NA objective, OrcaAG CCD camera (Hamamatsu, Bridgewater, NJ), automated stage (Prior Scientific, Rockland, MA), and IPLab software (Scanalytics, Fairfax, VA).

\subsubsection{Quantifying changes in shape}

After initial images were collected, myocytes were rinsed and cultured in serum-free media containing one of three concentrations of a hypertrophic agonist (dilution factor $=10$, intermediate concentration listed): $1 \mu \mathrm{M}$ Atrial Natriuretic Factor (ANF), $1 \mu \mathrm{M}$ Angiotensin II (AngII), $1 \mathrm{nM}$ Cardiotrophin-1 (CT1), 10 nM Epidermal Growth Factor (EGF), 100 nM Endothelin-1 (ET1 while maintaining cell health in the serum free control condition), $20 \mathrm{ng} / \mathrm{mL}$ Fibroblast growth factor-2 (FGF2), 10 nM Insulin Growth Factor-1 (IGF1), 10 ng/mL Interleukin-6 (IL6), Isoproterenol (ISO), 1 nM Leukemia Inhibitory Factor (LIF), $1 \mu \mathrm{M}$ Norepinephrine (NE), 10 ng/mL Neuregulin-1 (Nrg1), $1 \mu$ M Phenylephrine (PE), $1 \mathrm{ng} / \mathrm{mL}$ Transforming Growth Factor-beta (TGF $\beta$ ), or $10 \mathrm{ng} / \mathrm{mL}$ Tumor Necrosis Factor-alpha (TNF $\alpha$ ). A comprehensive list of agonist concentrations is shown in Table 4.1. After 48 hours, myocytes were imaged again. 48 hour stimulation allowed for robust changes in cell size and shape to be measured while maintaining cell health in serum free conditions.

Changes in myocyte area, perimeter, form factor, elongation, and integrated fluorescence intensity were calculated using automated custom Matlab image analysis algorithms. Form factor is a measure of circularity and is calculated as $4 \pi^{*}$ area/perimeter ${ }^{2}$. A circle therefore has a form factor value of 1 and all other shapes have form factors less than 1 . Elongation is a ratio of the major axis length to the minor axis length of the myocyte. All shape measurements were recorded from two wells from three independent myocyte isolations. 


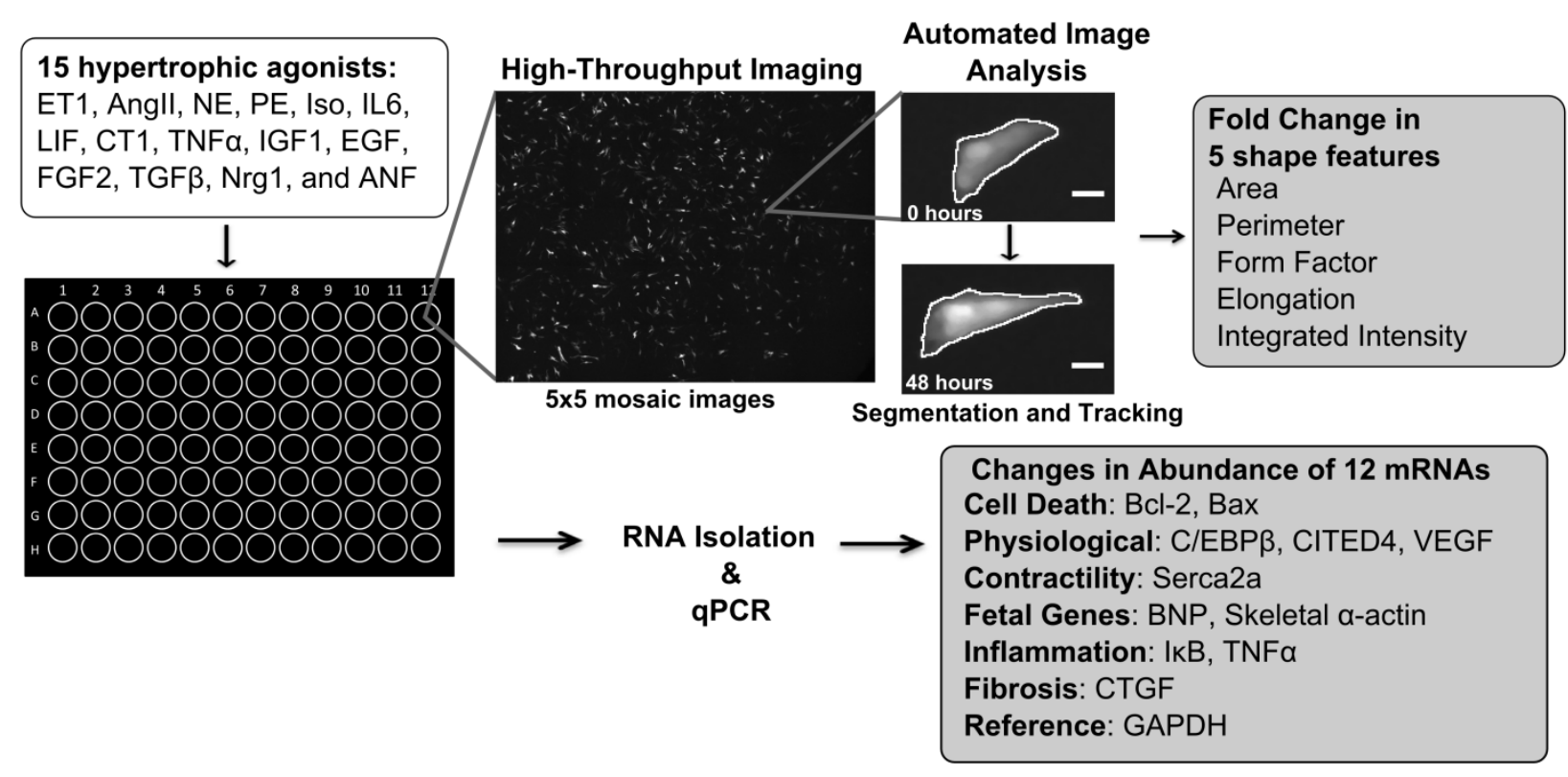

Figure 4.1: 15 ligand hypertrophic agonist screen in neonatal rat cardiac myocytes, measuring changes in 5 shape features and abundance of 12 mRNAs. Myocytes were treated for 48 hours with 1 of 15 hypertrophic agonists. Fold change in 5 shape features was calculated using our automated image acquisition and analysis platform. We measured fold change in abundance of $12 \mathrm{mRNAs}$ related to cell death, physiological hypertrophy, contractility, fetal genes, inflammation, and fibrosis using qPCR. 


\begin{tabular}{|c|c|c|c|c|}
\hline Ligand & Abbreviation & Low & Medium & High \\
\hline Atrial Natriuretic Factor & ANF & $0.1 \mu \mathrm{M}$ & $1 \mu \mathrm{M}$ & $10 \mu \mathrm{M}$ \\
\hline Angiotensin II & Ang II & $0.1 \mu \mathrm{M}$ & $1 \mu \mathrm{M}$ & $10 \mu \mathrm{M}$ \\
\hline Cardiotrophin-1 & CT1 & $0.1 \mathrm{nM}$ & $1 \mathrm{nM}$ & $10 \mathrm{nM}$ \\
\hline Epidermal Growth Factor & EGF & $1 \mathrm{nM}$ & $10 \mathrm{nM}$ & $100 \mathrm{nM}$ \\
\hline Endothelin-1 & ET1 & $10 \mathrm{nM}$ & $0.1 \mu \mathrm{M}$ & $1 \mu \mathrm{M}$ \\
\hline Fibroblast Growth Factor-2 & FGF2 & $2 \mathrm{ng} / \mathrm{mL}$ & $20 \mathrm{ng} / \mathrm{mL}$ & $200 \mathrm{ng} / \mathrm{mL}$ \\
\hline Insulin Growth Factor-1 & IGF1 & $1 \mathrm{nM}$ & $10 \mathrm{nM}$ & $100 \mathrm{nM}$ \\
\hline Interleukin-6 & IL6 & $1 \mathrm{ng} / \mathrm{mL}$ & $10 \mathrm{ng} / \mathrm{mL}$ & $100 \mathrm{ng} / \mathrm{mL}$ \\
\hline Isoproterenol & Iso & $0.1 \mu M$ & $1 \mu M$ & $10 \mu M$ \\
\hline Leukemia Inhibitory Factor & LIF & $0.1 \mathrm{nM}$ & $1 \mathrm{nM}$ & $10 \mathrm{nM}$ \\
\hline Norepinephrine & NE & $0.1 \mu M$ & $1 \mu \mathrm{M}$ & $10 \mu \mathrm{M}$ \\
\hline Neuregulin-1 & Nrg1 & $1 \mathrm{ng} / \mathrm{mL}$ & $10 \mathrm{ng} / \mathrm{mL}$ & $100 \mathrm{ng} / \mathrm{mL}$ \\
\hline Phenylephrine & PE & $0.1 \mu M$ & $1 \mu M$ & $10 \mu M$ \\
\hline Transforming Growth Factor- $\beta$ & TGF $\beta$ & $0.1 \mathrm{ng} / \mathrm{mL}$ & $1 \mathrm{ng} / \mathrm{mL}$ & $10 \mathrm{ng} / \mathrm{mL}$ \\
\hline Tumor Necrosis Factor- $\alpha$ & TNF $\alpha$ & $1 \mathrm{ng} / \mathrm{mL}$ & $10 \mathrm{ng} / \mathrm{mL}$ & $100 \mathrm{ng} / \mathrm{mL}$ \\
\hline
\end{tabular}

Table 4.1: Concentrations of ligands used in the hypertrophic agonist screen. Changes in shape were measured at all 3 concentrations and changes in mRNA abundance were measured at the medium concentration. 


\subsubsection{Quantifying changes in transcript abundance}

48 hours after stimulation with the hypertrophic agonists, total RNA was purified from myocytes given the intermediate concentration of the agonist using the RNeasy Mini kit (Qiagen, Valencia, CA). Complementary DNA was synthesized from $85.5 \mathrm{ng}$ of total RNA using the iScript cDNA synthesis kit (Bio-Rad). mRNA levels of twelve genes (Bcl-2, Bax, C/EBP $\beta$, CITED4, VEGF, Serca2a, BNP, Skeletal $\alpha$ actin, IKB, TNF $\alpha, C T G F$, and GAPDH) were measured using qPCR (BioRad CFX Connect) using iTaq Universal SYBR Green Supermix (Bio-Rad), 2 ng of cDNA, and $400 \mathrm{nM}$ of each primer set. GAPDH served as internal control. Gene-specific primers were designed on PrimerQuest (Integrated DNA Technologies, Inc.) A list of primers used is shown in Table 4.2. Data were analyzed using the comparative $C_{T}$ method with efficiency correction[108]. Measurements were collected from three independent myocyte isolations.

\subsection{4 siRNA knockdown:}

Two days after isolation, $10 \mathrm{nM}$ silencer select siRNA (Ambion) was transfected into cells using Lipofectamine RNAiMAX, as described by the manufacturer's protocol. Two siRNA sequences were tested for each target. Cells were transfected again the next day with $10 \mathrm{nM}$ siRNA in fresh medium. 24 hours following the second transfection, a given hypertrophic agonist was applied (0.1 $\mu \mathrm{M}$ Ang II, 10 $\mathrm{ng} / \mathrm{mL} \mathrm{Nrg1}$, or $1 \mathrm{nM}$ LIF). 48 hours after addition of the agonist, RNA was purified from the myocytes as described above. For CITED4 knockdown, Lipofectamine 2000 was used with the first transfection so that the cTnt-GFP plasmid could be introduced simultaneously for subsequent imaging.

\subsubsection{CITED4 adenoviral overexpression}

To express CITED4 in neonatal rat ventricular myocytes the full-length cDNA for CITED4 with either an $\mathrm{N}$ or C-terminal Flag tag was cloned into the pENTR vector (Life Technologies) and subsequently 


\begin{tabular}{|c|c|c|l|l|}
\hline Gene & NCBI Gene ID & Species & \multicolumn{1}{|c|}{ Forward primer } & \multicolumn{1}{c|}{ Reverse Primer } \\
\hline BAX & 24887 & Rat & TTTGCAGACGGCAACTTCAACTGG & TGTCCAGCCCATGATGGTTCTGAT \\
\hline BCL2 & 24224 & Rat & TTGTGGCCTTCTTTGAGTTCGGTG & TCATCCACAGAGCGATGTTGTCCA \\
\hline BNP & 25105 & Rat & TCTCAAAGGACCAAGGCCCTACAA & CTTCTGCCCAAAGCAGCTTGAACT \\
\hline C/EBPb & 24253 & Rat & TGATGCAATCCGGATCAAACGTGG & TTTAAGTGATTACTCAGGGCCCGGCT \\
\hline CITED4 & 114491 & Rat & TTTGCAGTCTGCTACTACTGCGG & TCGGATTTGTTCACTGTCTCACGC \\
\hline CTGF & 64032 & Rat & ACATGGCGTAAAGCCAGGGAGTAA & ATGACACACGGTTCTCACTTCGGT \\
\hline GAPDH & 24383 & Rat & TTGGCTCTATTGGGTGCCTGGTTA & AGCTTCCCATTCTCAGCCTTGACT \\
\hline IKB & 25493 & Rat & TCTGAAAGCTGGCTGTGATCCTGA & TAGACACGTGTGGCCGTTGTAGTT \\
\hline SERCA2a & 29693 & Rat & TGGTAGCCAATGCAATTGTGGGTG & TCCTGTCGATACACCTTGCCCATT \\
\hline Skeletal $\alpha-a c t i n$ & 29437 & Rat & ATCTATGAGGGTTATGCCCTGCCA & TTTGATGTCGCGCACAATCTCACG \\
\hline TNF $\alpha$ & 24835 & Rat & AGAACAGCAACTCCAGAACACCCT & TGCCAGTTCCACATCTCGGATCAT \\
\hline VEGFa & 83785 & Rat & TGAAAGACTCCGGTGTGGTCT & GTTTCTGGAAGTGAGCCAACG \\
\hline
\end{tabular}

Table 4.2: List of primer sequences used for measurements of changes in mRNA abundance in the hypertrophic agonist screen. Primers were designed using PrimerQuest (Integrated DNA Technologies). 
subcloned into an adenoviral destination vector. Transfection into HEK 293 cells resulted in significant virus production within one week. After purification the viral titer was determined by immunocytochemistry to viral coat proteins (Adeno-X, Clontech). Neonatal rat cardiac myocytes cultured on laminin-coated coverslips were treated with adenoviral constructs expressing CITED4 or lacZ for 12 hours. Myocytes were then cultured in serum free media containing the thymidine analogue EdU $(10 \mu \mathrm{M})$ for an additional 24 hours before imaging. A multiplicity of infection (MOI) of 30 was used for overexpression experiments. After fixation cultures were stained with antibodies against sacromeric actin ( $\alpha$-actinin) to identify cardiac myocytes and antibodies for Ki67 or EdU detection for determination of proliferation. Myocytes were imaged with a Zeiss 510 laser scanning confocal microscope with a 40X objective. 60 high power frames (HPFs) were acquired and analyzed from 3 independent experiments.

\subsection{Results}

\subsubsection{Differential effects on myocyte size and shape}

Our automated microscopy and image analysis platform[39] enables quantitative comparison of changes in area, elongation, form factor, perimeter, and integrated fluorescence intensity between signaling pathways (Figure 4.1). Fold changes in each of these shape features was calculated after 48 hour stimulation with three different concentrations of 15 hypertrophic agonists (Figure 4.2-4). These data reveal distinct regulation of shape among the 15 hypertrophic agonists. The $\alpha$-adrenergic agonists $\mathrm{PE}, \mathrm{NE}$, and ET1 caused the largest increases in cell area. While many of the agonists caused minimal changes to cell size, other shape changes were affected more dramatically. This is most evident with Nrg1 stimulation, which did not significantly affect cell area but caused the greatest increases in myocyte elongation. The $\alpha$-adrenergic agonists ET1, NE, and PE decreased form factor due to increases

in the number of cell protrusions. Nrg1 stimulation most dramatically increased elongation with LIF and FGF also causing high elongation responses at specific 

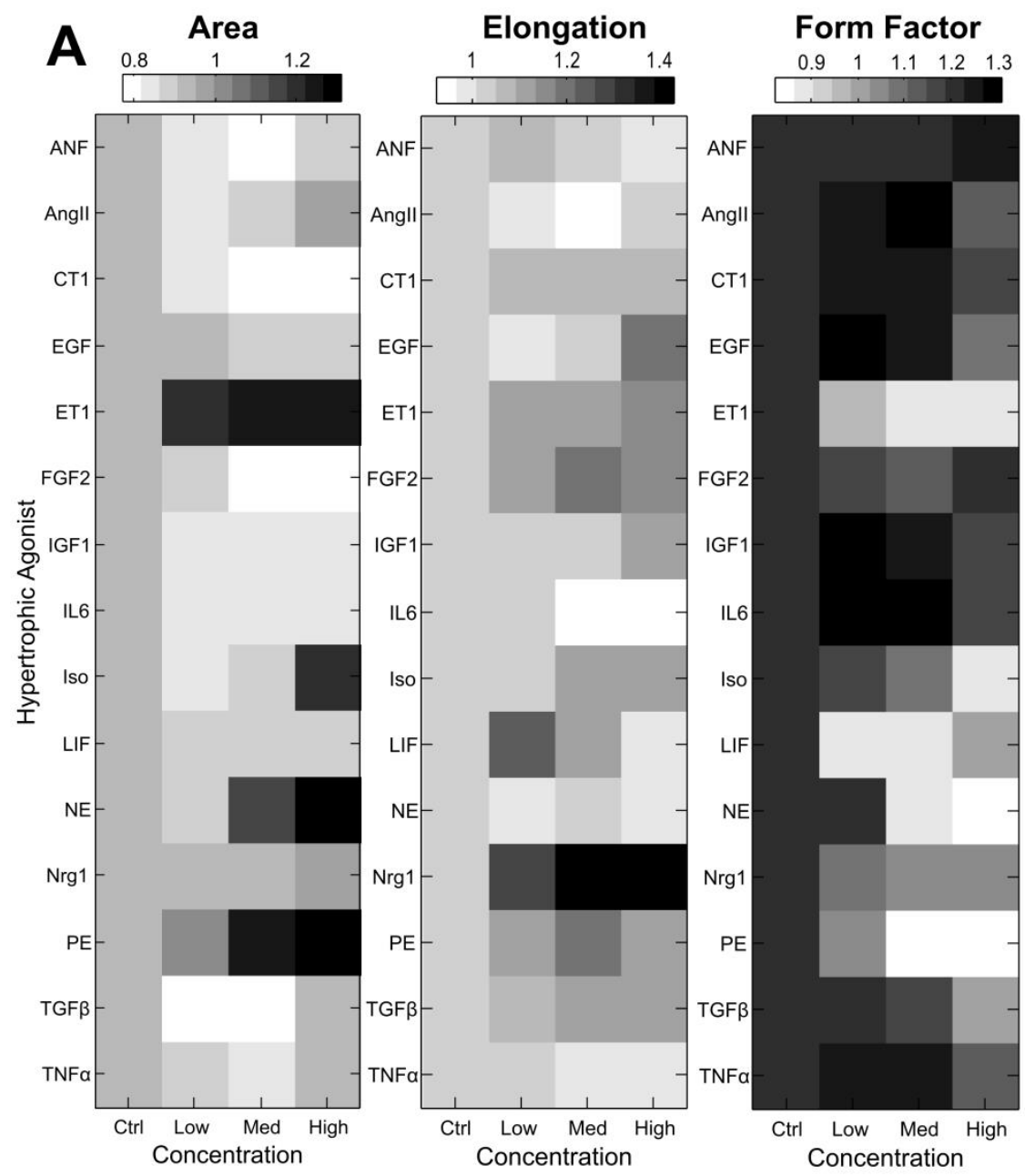

B
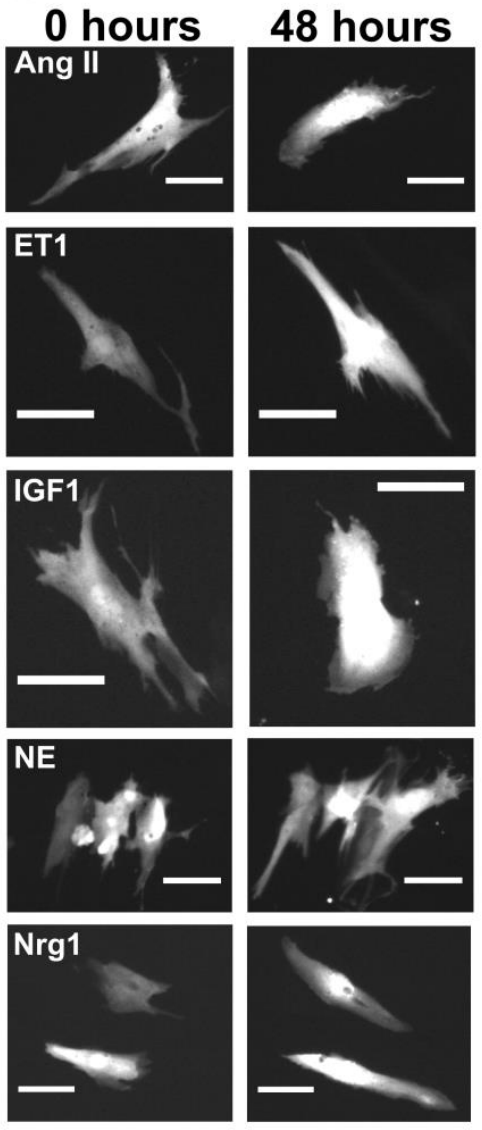

Figure 4.2: Hypertrophic agonist dose responses reveal diverse monophasic and biphasic effects on myocyte shape. A) Heat maps of median fold changes in area, elongation, and form factor induced by three different concentrations of hypertrophic agonists, with a dilution factor of 10. B) Representative images of cardiac myocytes treated with a subset of the hypertrophic agonists tested, revealing unique changes in shape among the hypertrophy pathways (Scale bar: $50 \mu \mathrm{m}$ ). 


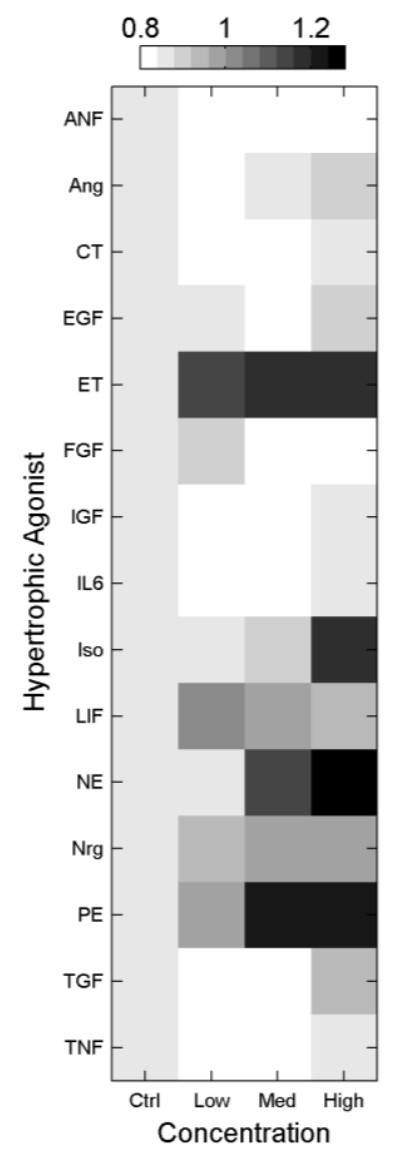

Figure 4.3: Hypertrophic agonist perimeter dose response data. Heat map of median fold change in perimeter induced by three different concentrations of hypertrophic agonists with a dilution factor of 10. Results qualitatively resemble cell area with an exception for LIF, which induced higher changes in perimeter than area due to increased elongation and decreased form factor. 


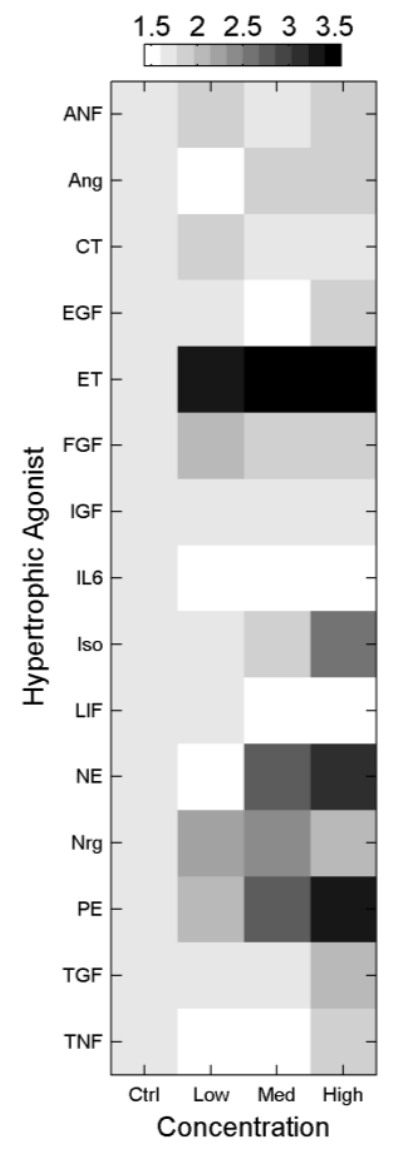

Figure 4.4: Hypertrophic agonist integrated fluorescence intensity dose response data. Heat map of median fold change in integrated intensity induced by three different concentrations of hypertrophic agonists with a dilution factor of 10. Results qualitatively resemble cell area with an exception for Nrg1, which induced higher changes in intensity than area. 
concentrations. Interestingly, many of the agonist dose responses revealed biphasic regulation of myocyte elongation, as can be seen with LIF, FGF, and PE. Representative myocyte images of the response to a subset of the hypertrophic agonists studied are shown in Figure $4.2 \mathrm{~B}$ and illustrate the diversity of shape responses observed among the agonists.

\subsubsection{Single cell data reveal differential regulation of shape features:}

Previous studies have hypothesized that due to the substantial cell-cell variability, single cell data from even control (untreated) conditions may contain valuable information about relationships between cellular phenotypes[109], [110]. To test this hypothesis, correlations between each of the five shape features were calculated using either the median response to the intermediate concentration of each hypertrophic agonist (Figure 4.5A) or single-myocyte data from control conditions ( $N=724$ myocytes) (Figure 4.5B).

Single cell data from untreated cells allowed identification of several interesting correlations in cell shape metrics. Area and elongation have a small correlation coefficient $(0.07)$, indicating that these features depict distinct features of cell shape and may be regulated by different pathways. As expected, area and perimeter are highly correlated (0.82) since these features both primarily describe cell size, however, some variation remains due to differences in shape. For example, LIF significantly increased perimeter without increasing cell area. Fluorescence intensity provides a reporter of troponin T promoter activity that, while highly correlated with cell size, is independent of cell area [39]. This correlation therefore indicates that larger myocytes typically have increased troponin T promoter activity. Form factor is moderately anti-correlated with both perimeter and elongation. Form factor is a measure of cell circularity, and therefore decreases with both elongation and increased numbers of cell protrusions. Form factor and area have a small correlation coefficient $(-0.26)$, indicating that these features capture distinct features and may be regulated by different pathways. Thus single-cell data 


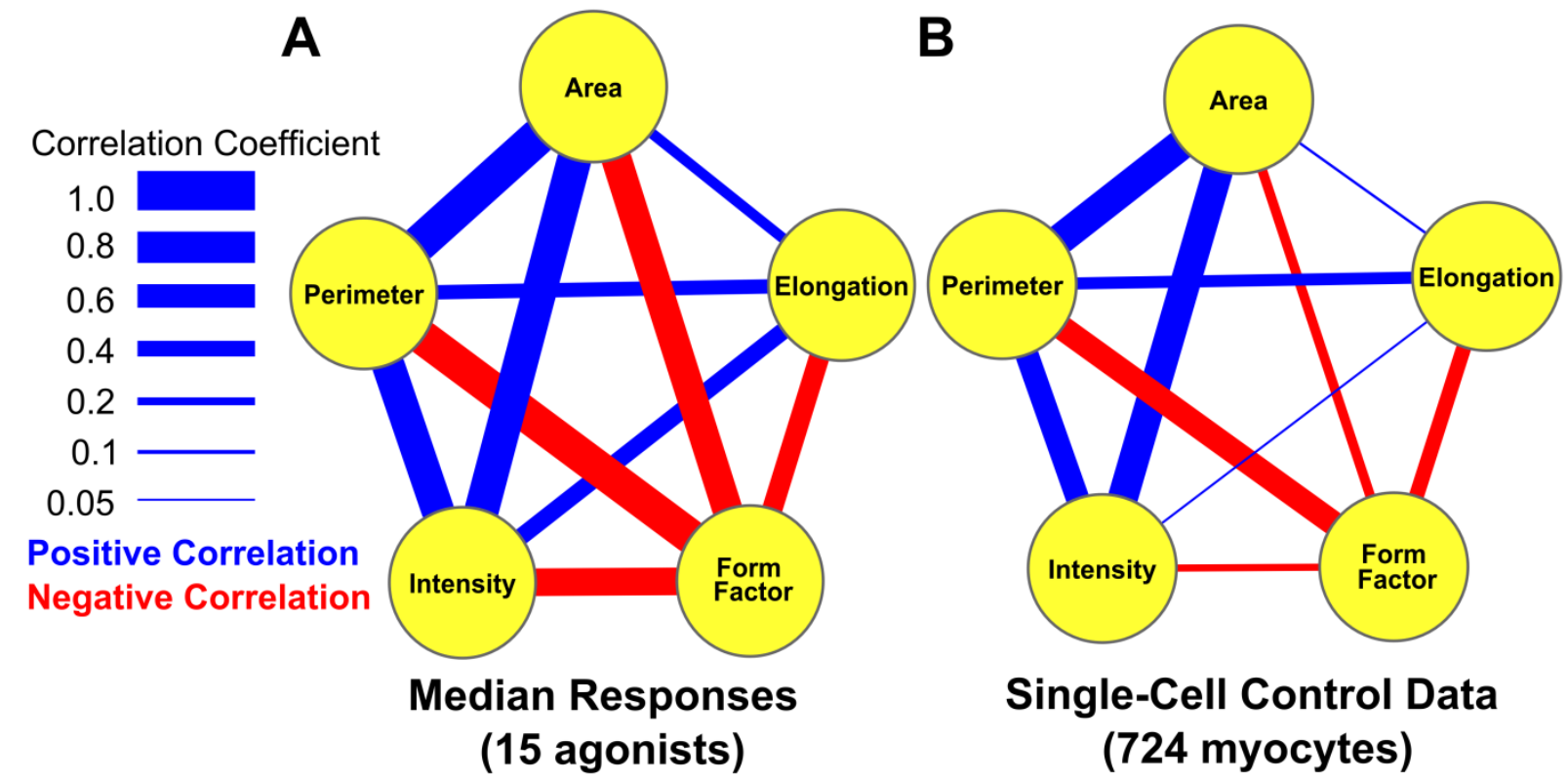

Figure 4.5: Intrinsic variability in single-cell data from untreated conditions is sufficient to capture distinct modes of myocyte shape regulation. Pearson correlation coefficients were calculated using either A) median fold change in shape feature data from myocytes stimulated with the intermediate concentration of each of the 15 agonists or B) single-cell data from the control myocytes ( $N=724$ myocytes) of fold change in shape features after 48 hours. 
from even a single treatment condition can harness cell-cell variability to reveal new relationships between myocyte phenotypes.

Correlations derived using the median responses to receptor agonists were qualitatively similar, but higher in magnitude than the correlations derived using single-cell control data. Median-derived correlations coefficients are larger since the agonists that highly affected cell size in this data set also typically had larger effects on many of the other shape metrics. For example, the $\alpha$-adrenergic agonists had the largest effects on cell area, perimeter, intensity, and form factor.

\subsubsection{Differential effects on myocyte transcript abundance}

For each of the 15 hypertrophic agonists, we measured fold change in mRNA abundance of 12 genes related to cardiac myocyte hypertrophy. Genes were selected to capture a diverse set of maladaptive and adaptive features of hypertrophy for each agonist. We measured mRNA abundance of genes previously related to cell death (Bax, which promotes apoptosis and necrosis[111], and antiapoptotic $\mathrm{Bcl} 2$ [112], [113]), physiological hypertrophy (C/EBP $\beta$, which is down-regulated with exercise [28], CITED4, which is upregulated with exercise and associated with myocyte proliferation [28], and pro-angiogenic VEGF [114]), contractility (Serca2a [115]), fetal genes (BNP and skeletal $\alpha$-actin [116]), inflammation (IKB [117] and TNF $\alpha$ [118], [119]), and fibrosis (connective tissue growth factor, CTGF [120]).

We performed hierarchical clustering of the hypertrophic agonist screen data of fold change in mRNA abundance and shape compared to control of myocytes treated with the intermediate concentration of each agonist (Figure 4.6). Each output (column), was normalized before clustering to give a mean of 0 and a standard deviation of 1 . This allowed us to compare outputs that had different effect sizes. Bar graphs showing data before normalization with standard error and $p$-values for each output is provided in the supplement (Figure 4.7-22). 


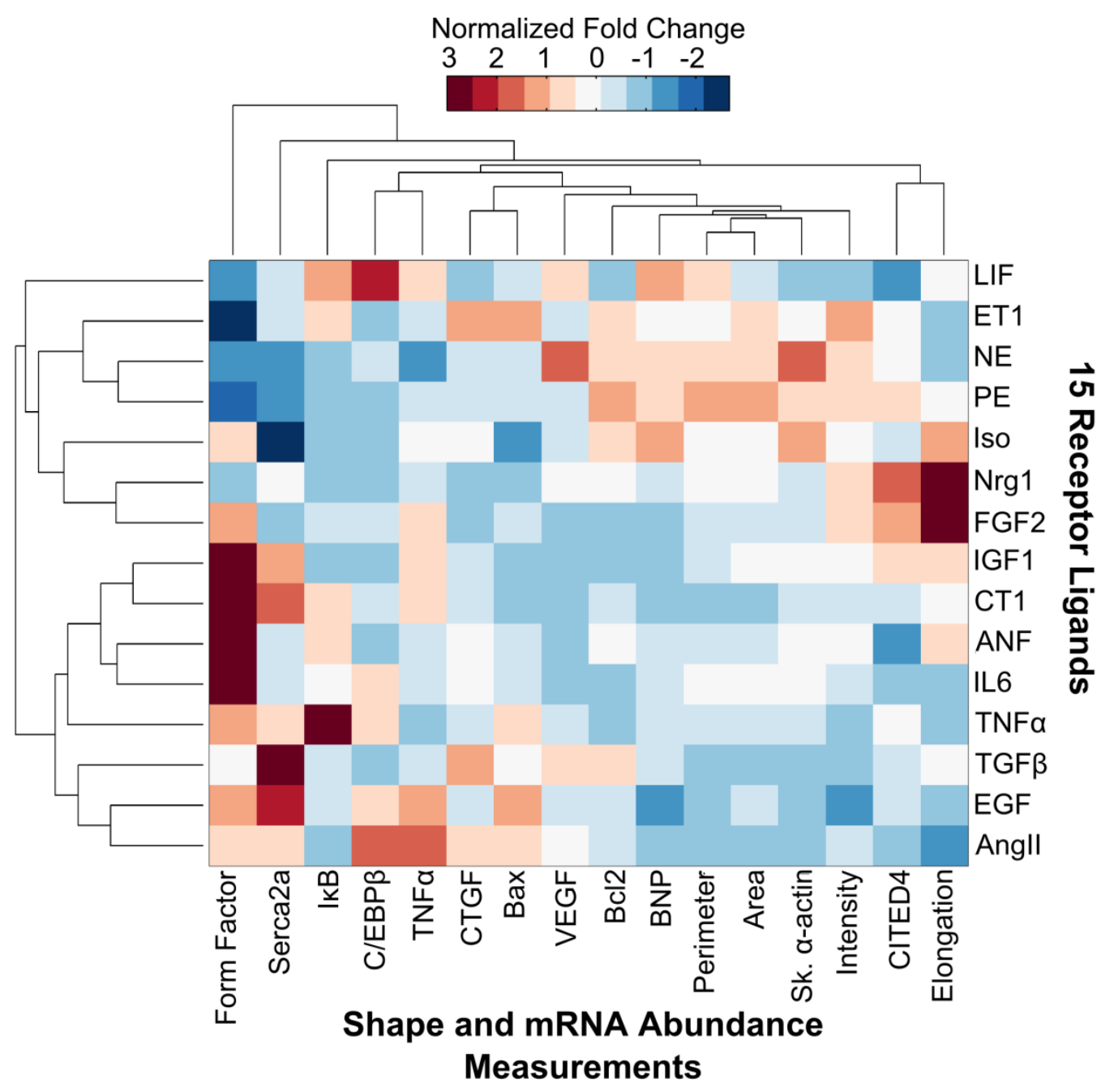

Figure 4.6: Agonist screen identifies distinct hypertrophy phenotypic signatures. Hierarchical clustering of the ligand screen data of fold change in output compared to control. Each output (columns) was normalized before clustering to give a mean of 0 and a standard deviation of 1 . Red indicates an agonist generates relatively high levels of a given output compared to the other agonists tested and blue indicates relatively low levels. While each agonist significantly affected at least one of the hypertrophic phenotypic outputs, agonists had unique signatures of hypertrophy. 


\section{Bax}

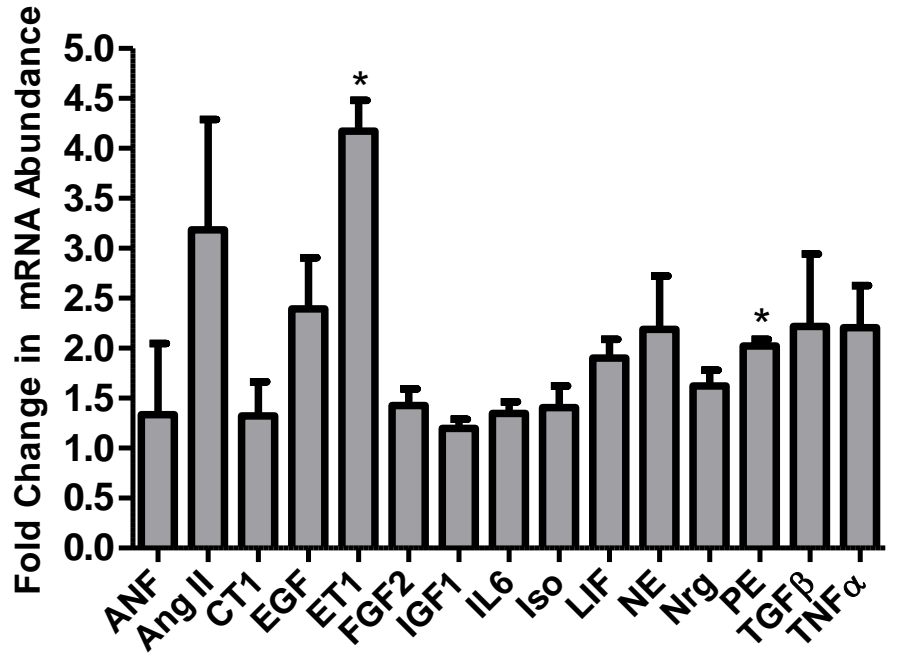

Hypertrophic Agonist

Figure 4.7: Fold change in Bax mRNA compared to control. Experiments were performed from three independent myocyte isolations and presented as mean $\pm S E M .{ }^{*} P<0.05$; unpaired, two-tailed Student's t-test.

\section{BCL2}

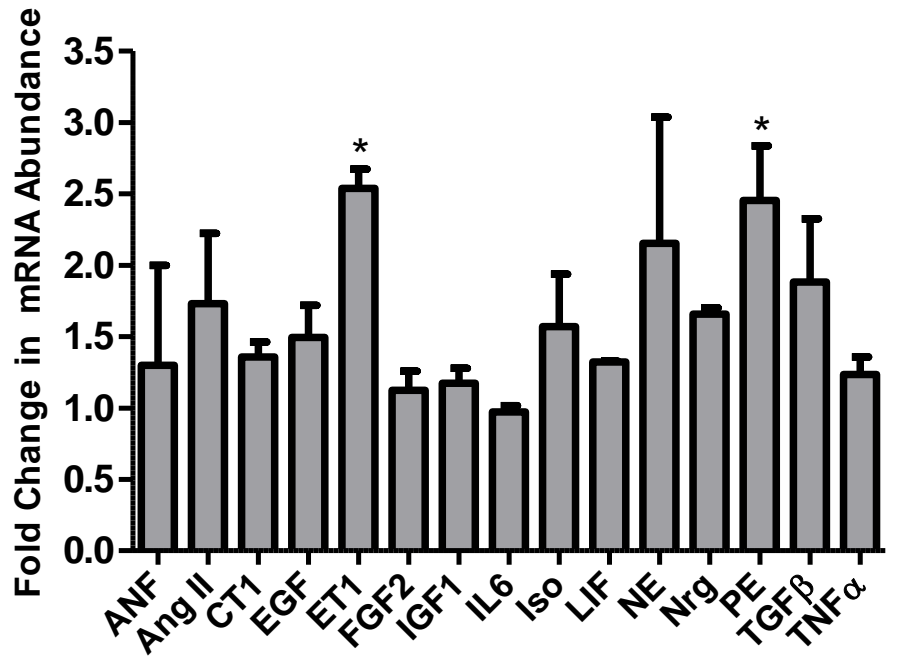

Hypertrophic Agonist

Figure 4.8: Fold change in Bcl2 mRNA compared to control. Experiments were performed from three independent myocyte isolations and presented as mean \pm SEM. ${ }^{*} \mathrm{P}<0.05$; unpaired, two-tailed Student's t-test. 


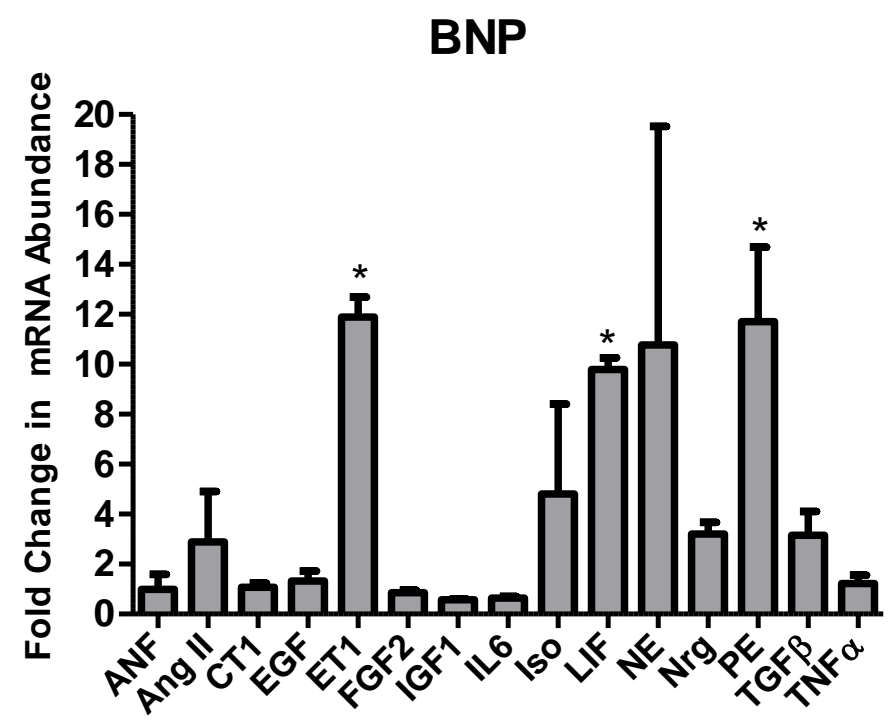

Hypertrophic Agonist

Figure 4.9: Fold change in BNP mRNA compared to control. Experiments were performed from three independent myocyte isolations and presented as mean $\pm S E M$. ${ }^{*} P<0.05$; unpaired, two-tailed Student's t-test.

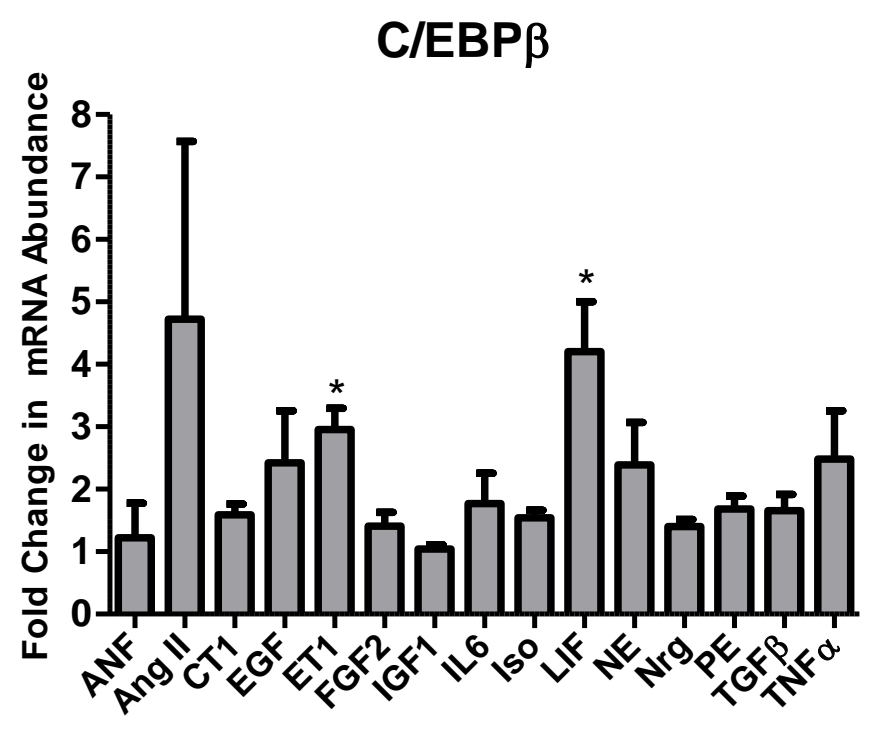

Hypertrophic Agonist

Figure 4.10: Fold change in C/EBP $\beta$ mRNA compared to control. Experiments were performed from three independent myocyte isolations and presented as mean \pm SEM. ${ }^{*} \mathrm{P}<0.05$; unpaired, two-tailed Student's t-test. 


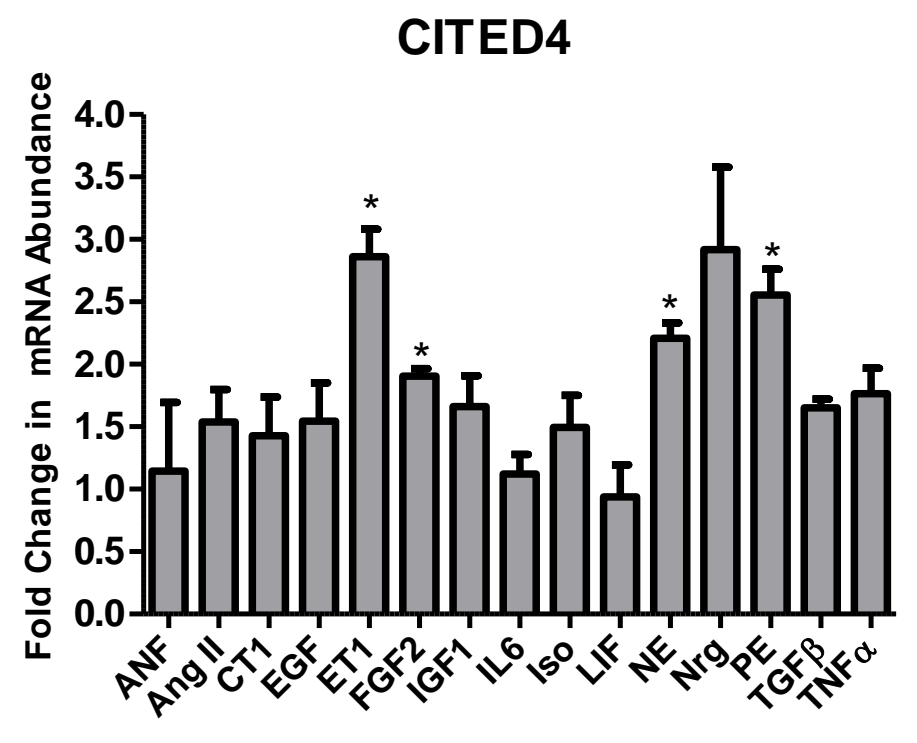

Hypertrophic Agonist

Figure 4.11: Fold change in CITED4 mRNA compared to control. Experiments were performed from three independent myocyte isolations and presented as mean $\pm \mathrm{SEM}$. ${ }^{*} \mathrm{P}<0.05$; unpaired, two-tailed Student's t-test.

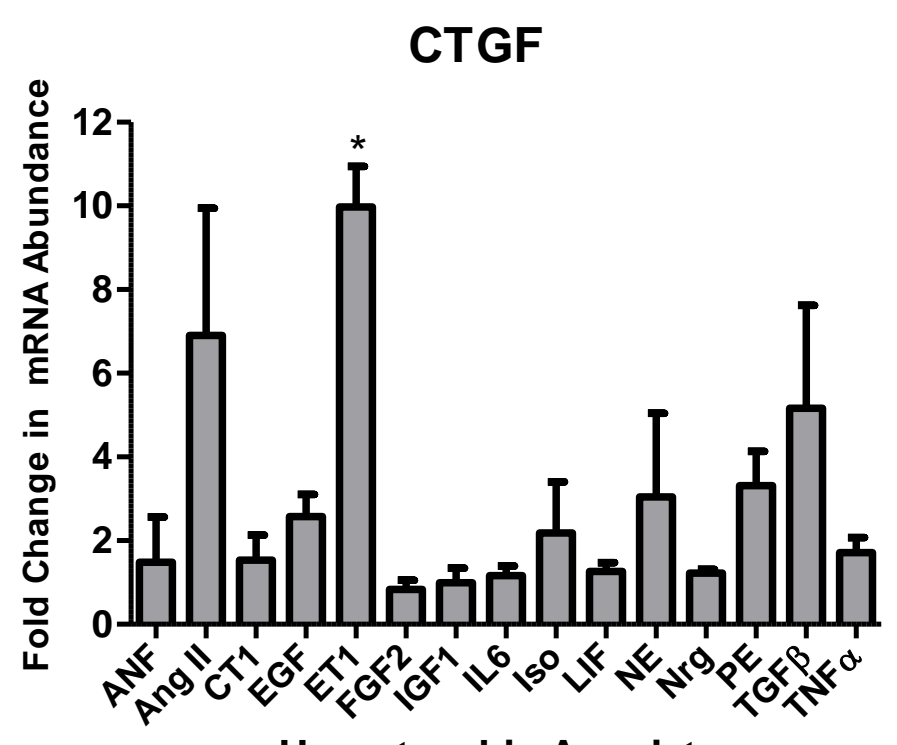

Hypertrophic Agonist

Figure 4.12: Fold change in CTGF mRNA compared to control. Experiments were performed from three independent myocyte isolations and presented as mean \pm SEM. ${ }^{*} \mathrm{P}<0.05$; unpaired, two-tailed Student's t-test. 


\section{IKB}

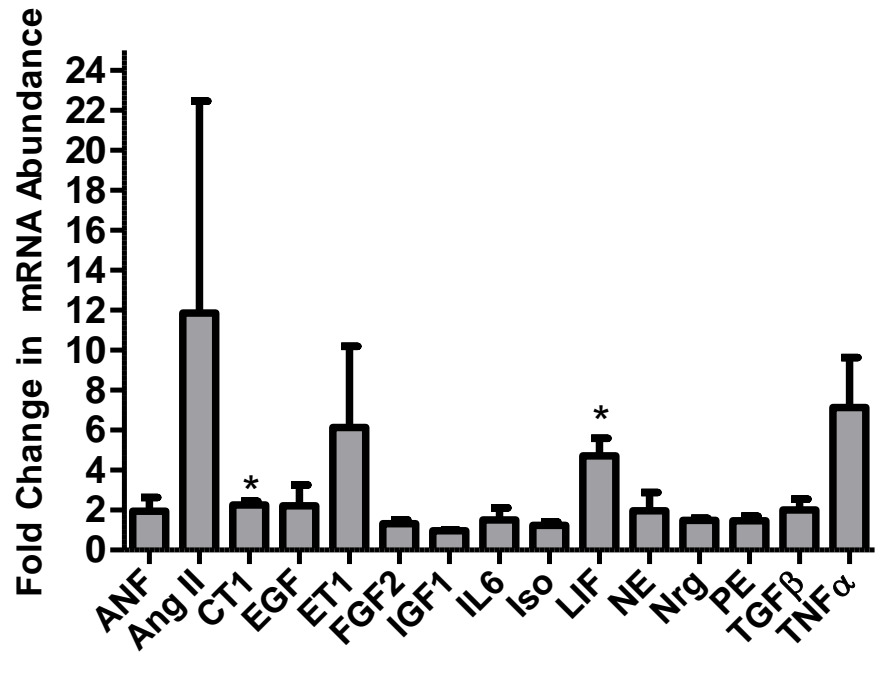

Hypertrophic Agonist

Figure 4.13: Fold change in IKB mRNA compared to control. Experiments were performed from three independent myocyte isolations and presented as mean $\pm S E M$. ${ }^{*} P<0.05$; unpaired, two-tailed Student's t-test.

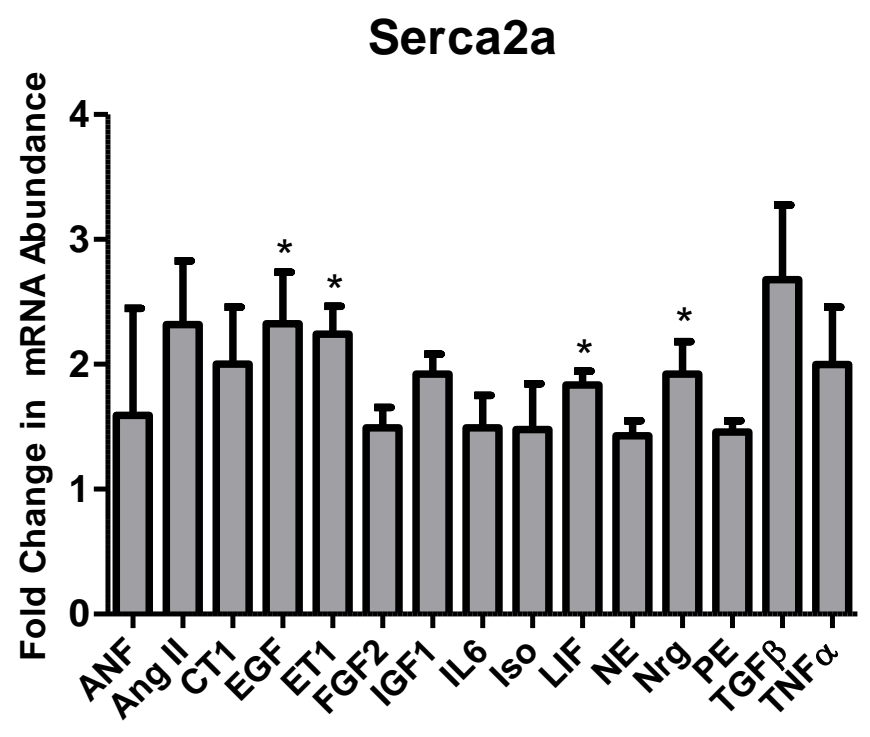

Hypertrophic Agonist

Figure 4.14: Fold change in Serca2a mRNA compared to control. Experiments were performed from three independent myocyte isolations and presented as mean \pm SEM. ${ }^{*} \mathrm{P}<0.05$; unpaired, two-tailed Student's t-test. 


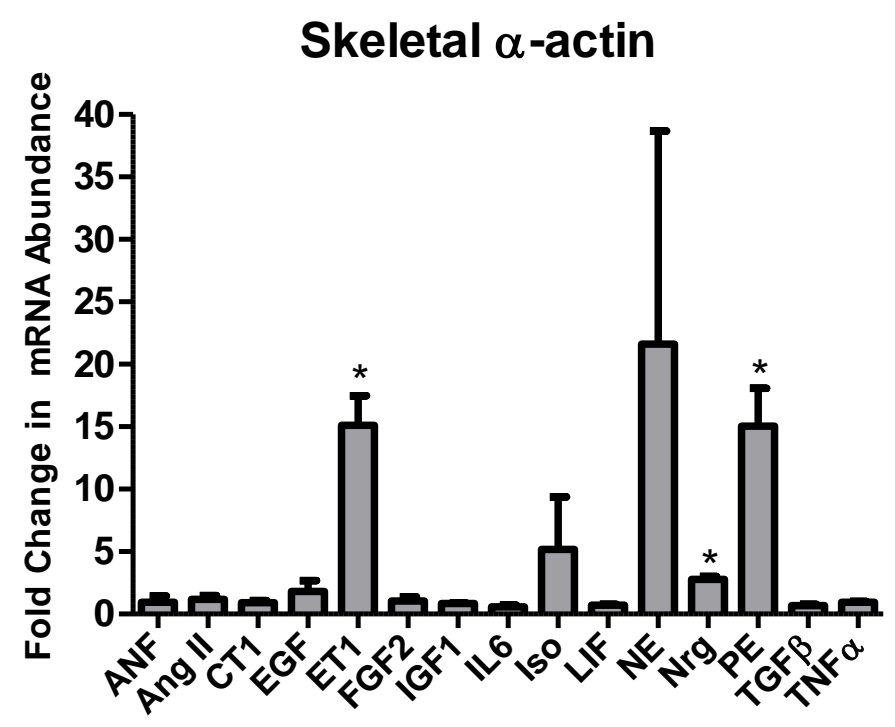

Hypertrophic Agonist

Figure 4.15: Fold change in Skeletal $\boldsymbol{\alpha}$-actin mRNA compared to control. Experiments were performed from three independent myocyte isolations and presented as mean $\pm S E M$. ${ }^{*} P<0.05$; unpaired, twotailed Student's t-test.

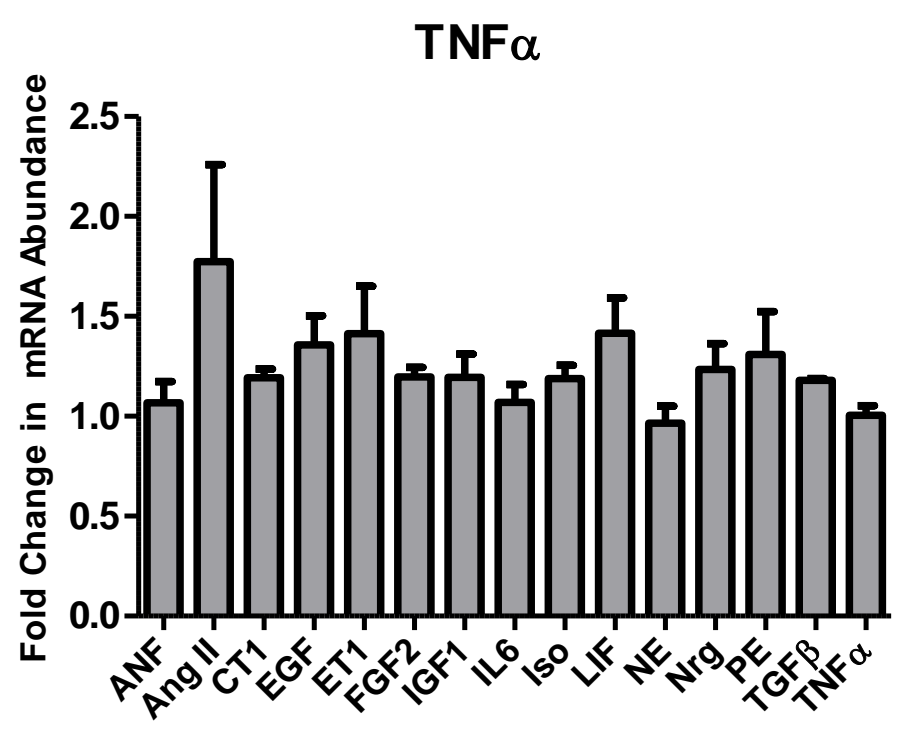

Hypertrophic Agonist

Figure 4.16: Fold change in TNF $\alpha$ mRNA compared to control. Experiments were performed from three independent myocyte isolations and presented as mean $\pm \mathrm{SEM} .{ }^{*} \mathrm{P}<0.05$; unpaired, two-tailed Student's t-test. 


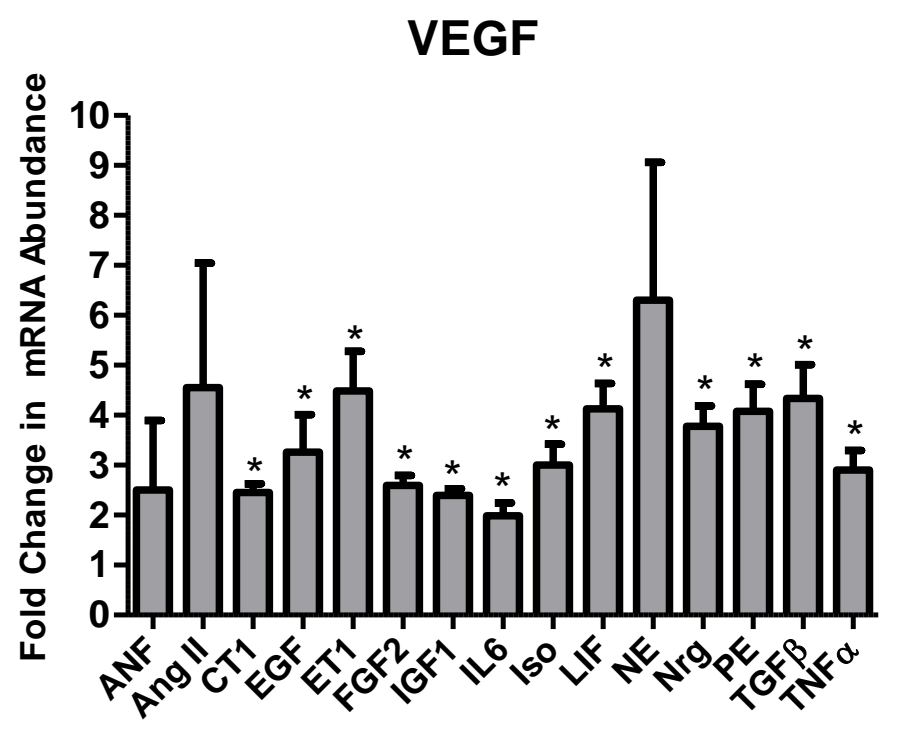

Hypertrophic Agonist

Figure 4.17: Fold change in VEGF mRNA compared to control. Experiments were performed from three independent myocyte isolations and presented as mean $\pm S E M$. ${ }^{*} P<0.05$; unpaired, two-tailed Student's t-test.

Area

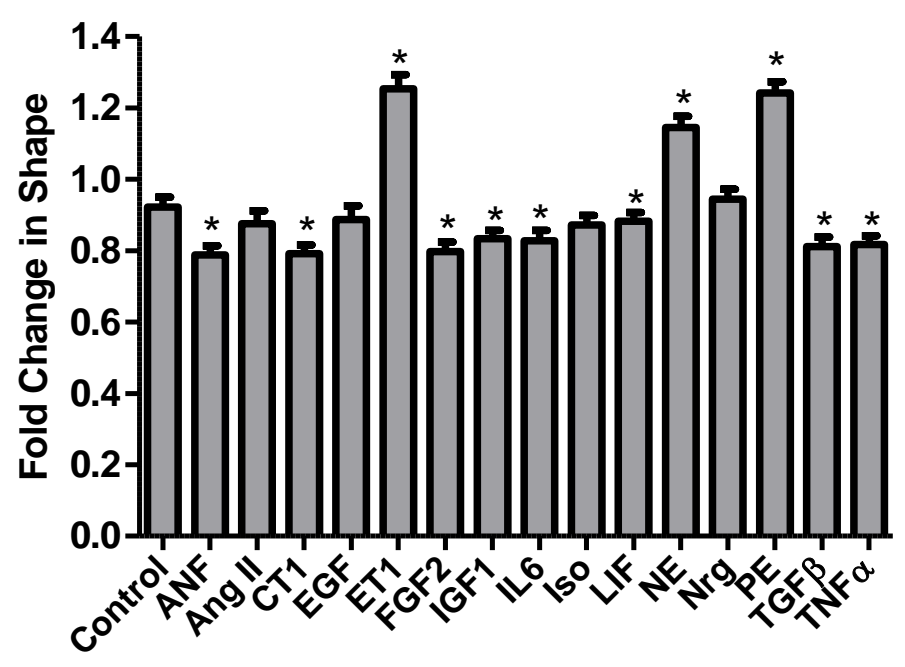

Hypertrophic Agonist

Figure 4.18: Fold change in myocyte area. Experiments were performed in two wells from three independent myocyte isolations ( 600 cells per condition) and presented as median \pm SEM. ${ }^{*} \mathrm{P}<0.05$; unpaired, two-tailed Student's t-test. 


\section{Elongation}

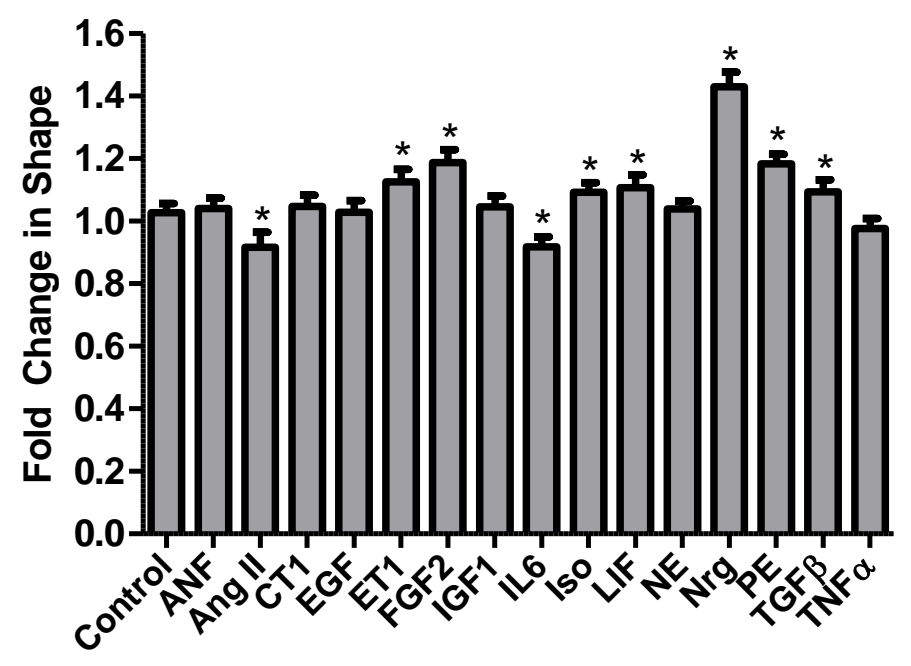

Hypertrophic Agonist

Figure 4.19: Fold change in myocyte elongation. Experiments were performed in two wells from three independent myocyte isolations ( $\sim 60$ cells per condition) and presented as median \pm SEM. ${ }^{*} P<0.05$; unpaired, two-tailed Student's t-test.

\section{Form Factor}

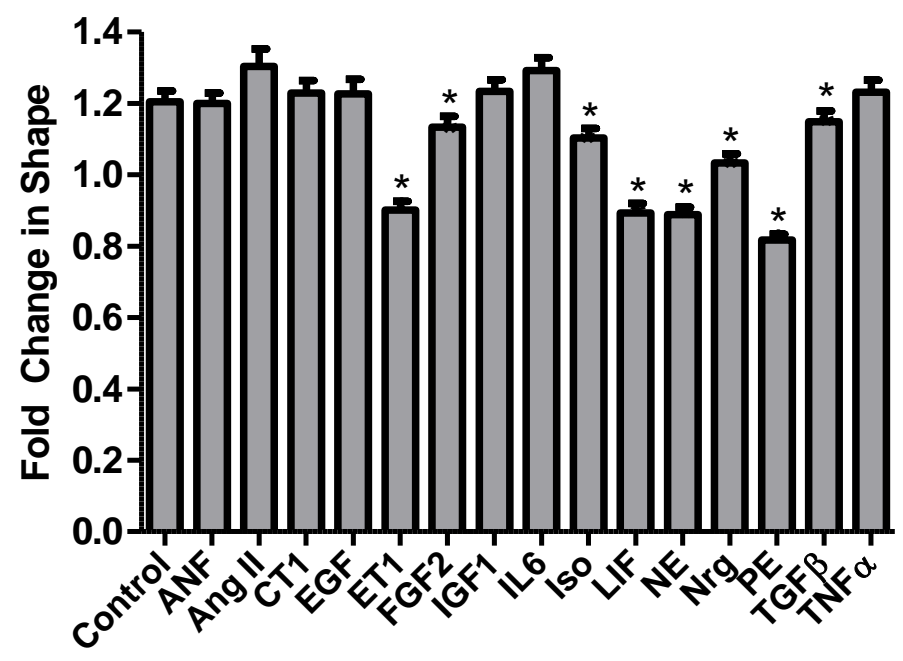

Hypertrophic Agonist

Figure 4.20: Fold change in myocyte form factor. Experiments were performed in two wells from three independent myocyte isolations ( $\sim 60$ cells per condition) and presented as median \pm SEM. ${ }^{*} \mathrm{P}<0.05$; unpaired, two-tailed Student's t-test. 


\section{Perimeter}

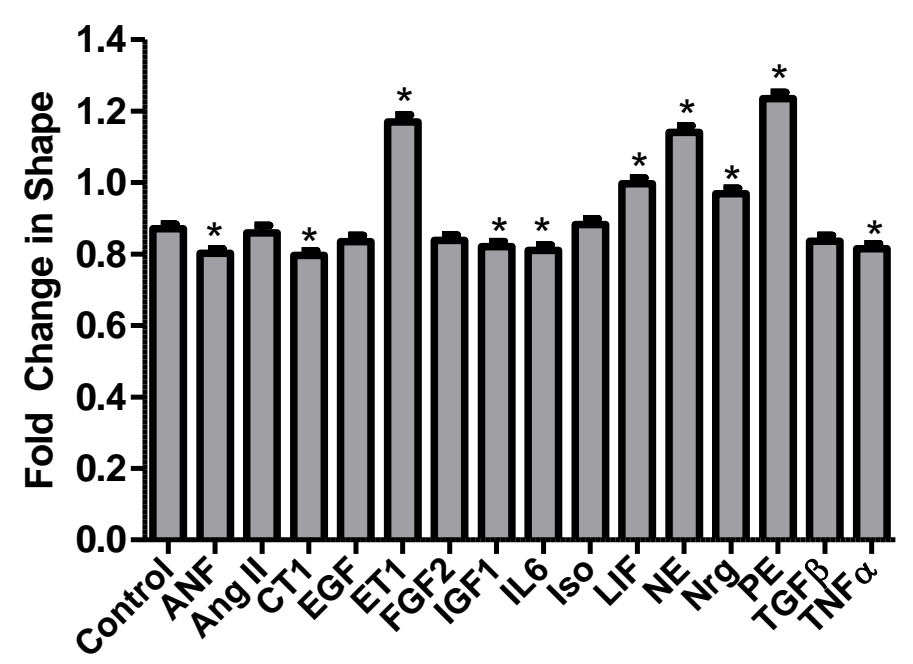

Hypertrophic Agonist

Figure 4.21: Fold change in myocyte perimeter. Experiments were performed in two wells from three independent myocyte isolations ( $\sim 60$ cells per condition) and presented as median $\pm \mathrm{SEM} .{ }^{*} \mathrm{P}<0.05$; unpaired, two-tailed Student's t-test.

\section{Fluorescence Intensity}

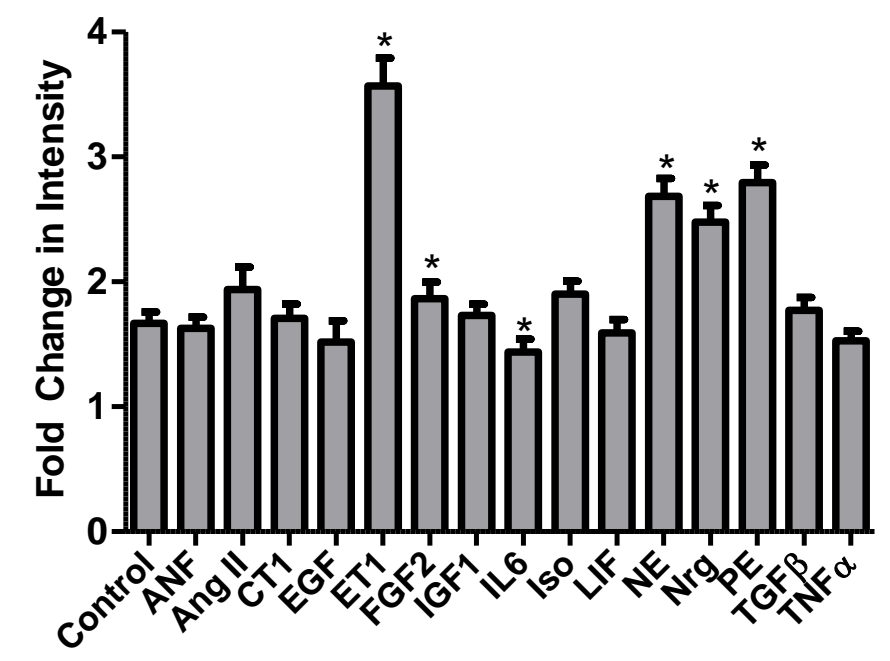

Hypertrophic Agonist

Figure 4.22: Fold change in myocyte integrated fluorescence intensity. Experiments were performed in two wells from three independent myocyte isolations ( $\sim 60$ cells per condition) and presented as median \pm SEM. ${ }^{*} \mathrm{P}<0.05$; unpaired, two-tailed Student's t-test. 
The combined mRNA abundance and shape data reveal that the hypertrophic agonists generate distinct phenotypic signatures of hypertrophy (Figure 4.6). CITED4 and elongation were notably upregulated by Nrg1 and FGF2, CTGF was upregulated by ET1, Ang II, and TGF $\beta$, Bax was upregulated by ET1, Ang II, and EGF, and C/EBPß was upregulated with Ang II and LIF. Remarkably, while many of the agonists had minimal effects on cell area, the effects on other hypertrophy phenotypic outputs were much more prominent. For example, while LIF did not significantly increase myocyte area, LIF led to significant increases in BNP, C/EBP $\beta$, and IKB. Moreover, nearly every agonist significantly upregulated VEGF mRNA abundance, which is important for myocyte growth without cardiac dysfunction [121] (Figure 4.17). Hierarchical clustering revealed new relationships among the outputs measured, identifying correlations between CITED4 and Elongation, TNF $\alpha$ and C/EBP $\beta$, and CTGF and Bax. Notable correlations among inputs were identified between NE and PE, Nrg1 and FGF2, and EGF and Ang II.

\subsubsection{Clustering reveals modular input-output relationships}

Through k-means clustering of the data in Figure 4.6, we identified highly correlated input and output modules and a network map linking input modules to output modules (Figure 4.23). We identified five modules within the inputs and five modules within the outputs. Lines between species within a module indicate $\mathrm{P}<0.01$ for a given correlation. Interestingly, many maladaptive outputs grouped together in one of the modules: Bax, C/EBP $\beta$, Serca2a, TNF $\alpha$, and CTGF. More beneficial outputs, Bcl2 and VEGF, grouped together with fetal genes, BNP and skeletal $\alpha$-actin, and myocyte area, perimeter, and fluorescent intensity. Nrg1 and ET1 were each in a module by themselves, indicating especially unique regulation of hypertrophic outputs. CITED4 and Elongation were highly correlated and in a distinct module from the other outputs measured.

We also calculated the median normalized fold change in output between each input-output pair within an input module and an output module. Bold lines connecting input modules and output 


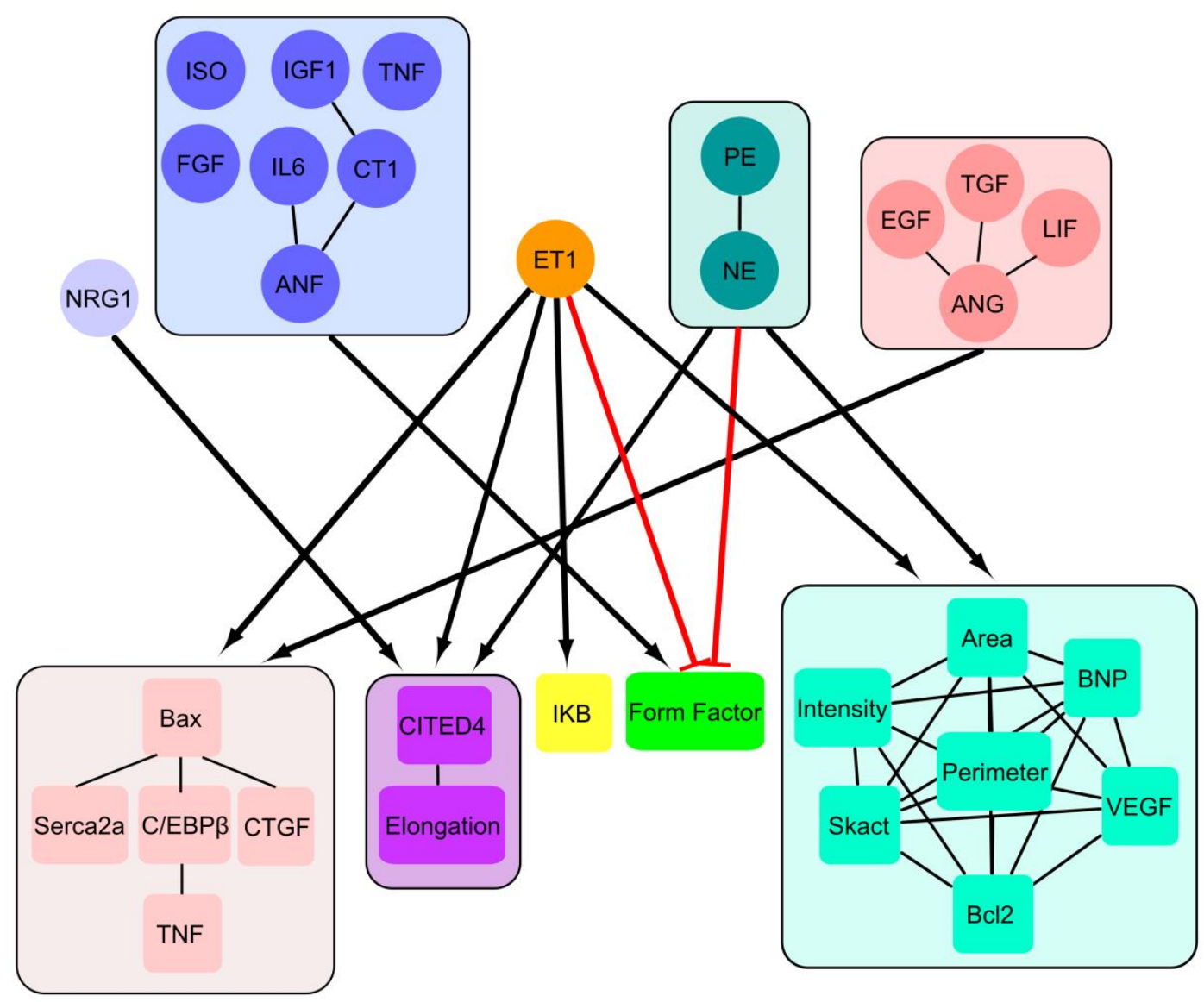

Figure 4.23: Clustering reveals modular input-output relationships in hypertrophy signaling. Diagram of the 5 input modules and 5 output modules generated by k-means clustering of the hypertrophic agonist screen data. Links between input modules and output modules are marked if the median normalized fold change in output between each input-output pair in the modules was $>|0.70|$ with red lines indicating negative effects. Lines marked between components within a module indicate $\mathrm{P}<.01$ from a given correlation. Clustering identified new relationships and modules among inputs and outputs, including correlations between CTGF and Bax and between CITED4 and myocyte elongation. 
modules were diagrammed (Figure 4.23) if the median normalized fold change in output between each input-output pair in the module was $>|0.7|$, with red lines indicating negative effects. The Ang II and ET1 modules had a high effect on the module with Bax and C/EBP $\beta$. ET1 had high effects on every output module, and was the only module to markedly affect IKB. The $\alpha$-adrenergic receptors had high effects on the module with cell size, fetal genes, and VEGF. Nrg1 upregulated the CITED4 and elongation module and the ANF module was the only group that increased form factor. This network map reveals shared regulation among maladaptive features of hypertrophy, which could enhance the development of pathological hypertrophy.

\subsubsection{Pro-fibrotic CTGF upregulates Bax mRNA abundance}

Given the maladaptive effects of myocyte death and fibrosis on cardiac hypertrophy, we wanted to further investigate our measured correlation between pro-cell death Bax and pro-fibrotic CTGF. While Bax/CTGF correlation could conceivably arise from a shared upstream pathway without causal relationships between Bax and CTGF, we hypothesized that CTGF mechanistically regulates Bax mRNA abundance in cardiac myocytes. To test this hypothesis, we performed a series of follow-up experiments. First, we confirmed the presence of CTGF in myocytes with immunofluorescence imaging (Figure 4.24-25).

We next tested whether recombinant CTGF was sufficient to increase Bax mRNA abundance. Five days after isolation, we applied 0.1 or $1 \mu \mathrm{M}$ recombinant human CTGF protein (Life Technologies) to the cardiac myocytes. After 48 hours we measured fold change in Bax mRNA using qPCR as described earlier. As hypothesized, application of recombinant CTGF to myocytes did lead to increased Bax mRNA abundance (Figure 4.26A). 
CTGF

Endothelin-1

Angtiotensin II

Control
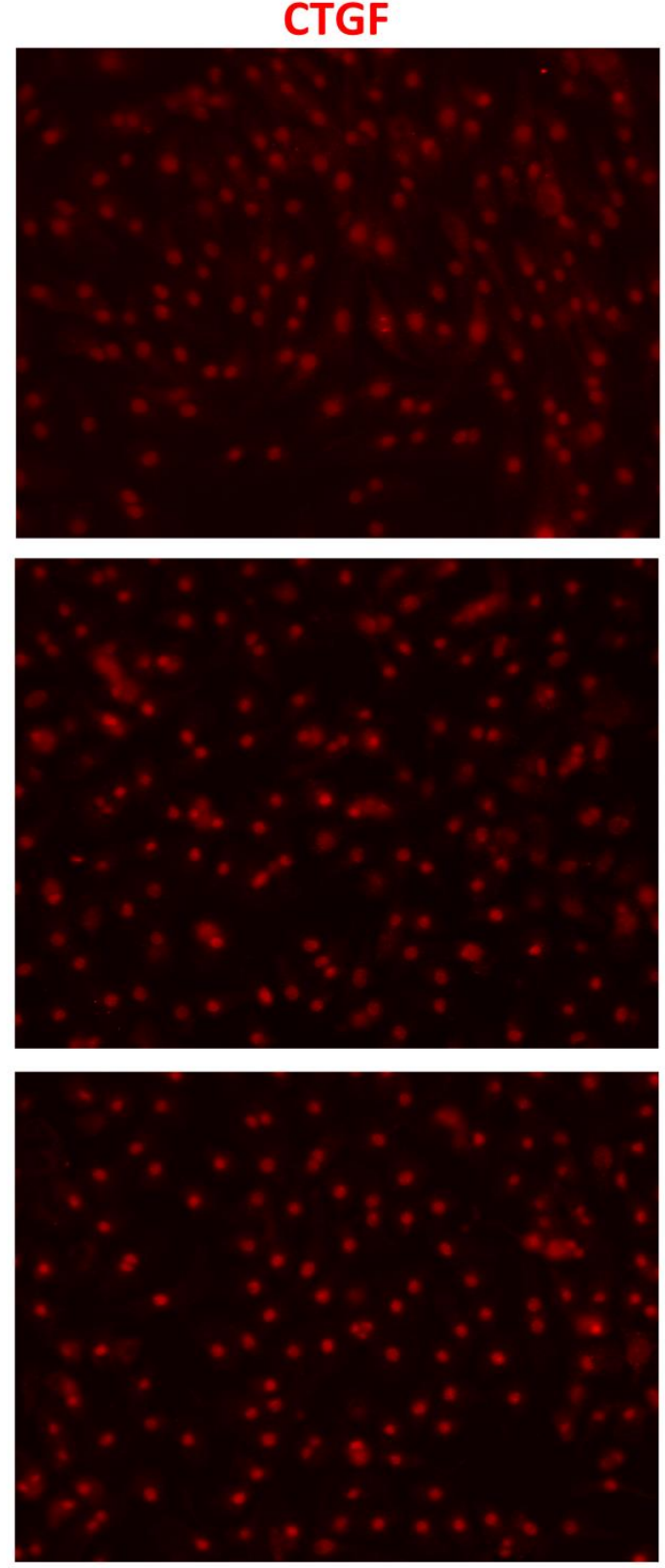

CTGF, $\alpha$-actinin, DAPI Merge
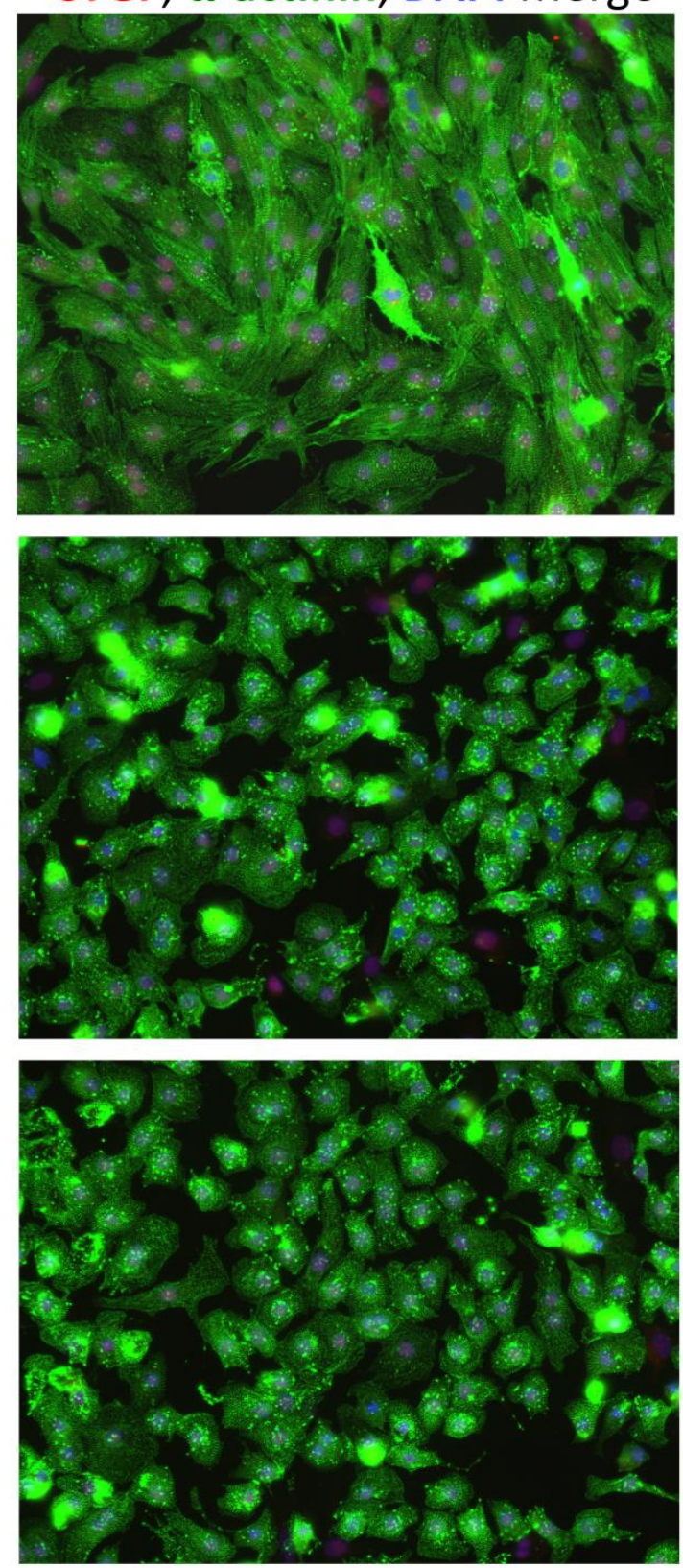

Figure 4.24: Immunofluorescence imaging of CTGF in neonatal rat ventricular cardiac myocytes. To ensure that fibrotic marker CTGF is present in cardiac myocytes, we labeled myocytes for $\alpha$-actinin and CTGF. 48-hours after isolation, myocytes were cultured with 100 nM ET1 or $10 \mu \mathrm{M}$ Ang II for 48-hours before fixing to induce upregulation of CTGF. Myocytes were labeled with mouse anti- $\alpha$-actinin (SigmaAldrich) and rabbit anti-CTGF (Abcam) primary antibodies (1:200 concentration), Alexa Fluor-488 goat anti-mouse (Invitrogen) and Alexa Fluor 594 goat anti-rabbit (Abcam) secondary antibodies (1:200 concentration), and DAPI with SlowFade (Invitrogen). CTGF was located predominantly in the nucleus and colocalized with cells expressing $\alpha$-actinin, indicating that CTGF is present in cardiac myocytes. 


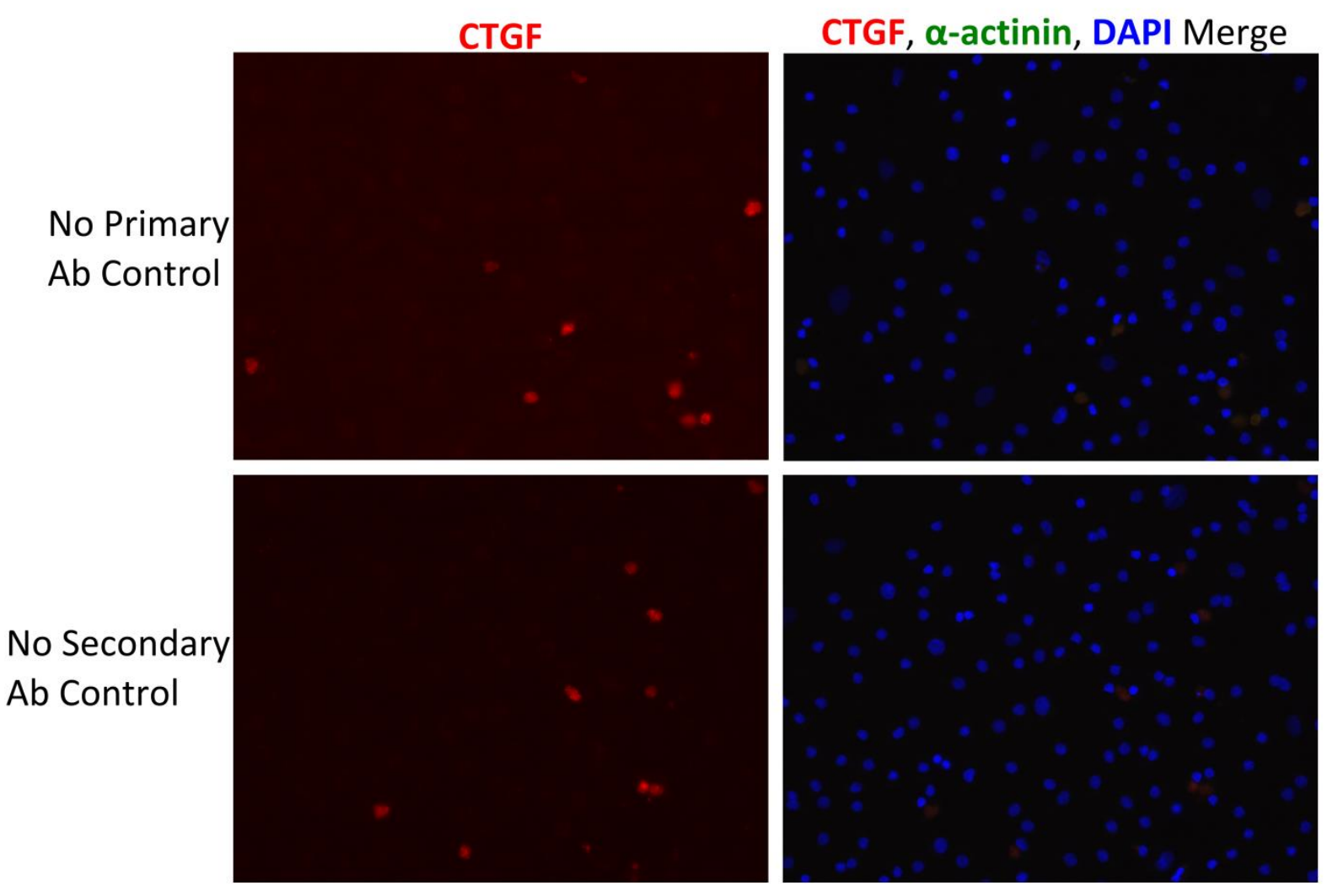

Figure 4.25: Immunofluorescence negative controls for imaging CTGF in neonatal rat ventricular cardiac myocytes. To ensure that fibrotic marker CTGF is present in cardiac myocytes, we labeled myocytes for $\alpha$-actinin and CTGF (Figure 4.24). Controls without primary antibodies (top) and secondary antibodies (bottom) reveal specificity of $\alpha$-actinin and CTGF immunofluorescence labeling. Nonspecific CTGF signals were only present in dead myocytes. 
A

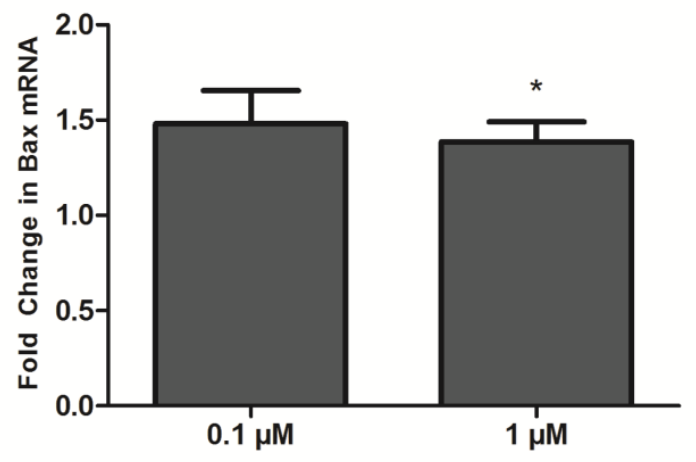

Recombinant CTGF concentration

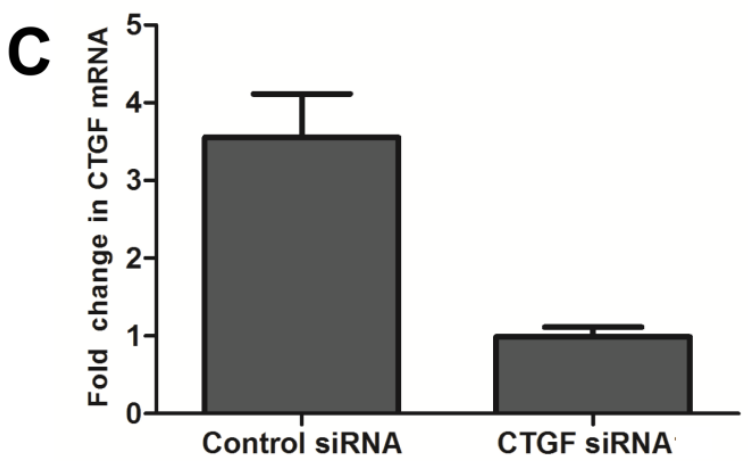

B

Ang II

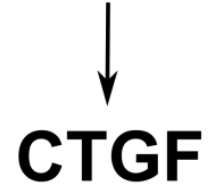

$\downarrow$

Bax mRNA

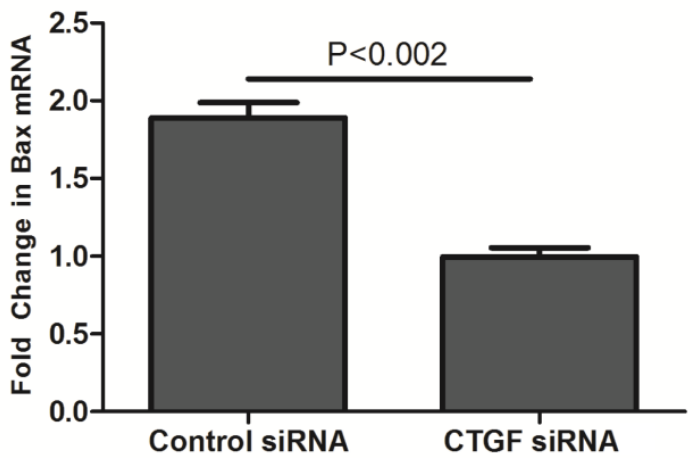

Figure 4.26: CTGF increases Bax mRNA abundance. A) Mean +/- SE fold change in Bax mRNA after 0.1 $\mu \mathrm{M}$ or $1 \mu \mathrm{M}$ recombinant CTGF was applied to myocytes for 48 hours. Bax mRNA abundance was quantified with qPCR. $1 \mu \mathrm{M}$ CTGF significantly increased the Bax mRNA level in cardiac myocytes $(P<.05$, Student's t-test). B) Schematic of hypothesized regulation of Bax mRNA by CTGF. Since CTGF induced increases in Bax mRNA in myocytes we hypothesized that regulation of Bax expression by CTGF may have led to the correlation between these features in our ligand screen data. $\mathrm{C}$ ) Mean +/- SE fold change in CTGF (left) and Bax (right) after CTGF mRNA was knocked down by siRNA transfection and myocytes were stimulated with $0.1 \mu \mathrm{M}$ Ang II for 48 hours. Knockdown of CTGF led to significantly decreased Bax mRNA compared to control siRNA ( $P<0.002$, Student's t-test). 
To further study the CTGF/Bax interplay under conditions mimicking pathological stress, we studied the effect of CTGF knockdown with siRNA on Bax mRNA abundance in Ang II-treated myocytes. Since recombinant CTGF increased Bax mRNA abundance, we hypothesized that knockdown of CTGF would lead to decreased Bax mRNA abundance. Ang II was chosen as an agonist because it induced large increases in abundance of the output module containing Bax and CTGF without greatly affecting many other outputs. As hypothesized, knockdown of CTGF decreased Ang II-dependent increases in Bax mRNA (Figure 4.26C). Together, these results indicate a signaling pathway from CTGF to Bax mRNA regulation in cardiac myocytes and reveal a role for CTGF in Ang II-induced increases in Bax mRNA abundance. While CTGF plays a significant role in Ang II-induced Bax regulation, Bax has other known regulators [122-125]. Thus in addition to promoting fibrosis, agonists causing increased CTGF may also enhance cell death signaling, potentially exacerbating maladaptive hypertrophic remodeling of the heart.

\subsubsection{CITED4 negatively regulates cardiac myocyte elongation}

Clustering analysis had revealed prominent correlation between CITED4 mRNA abundance and myocyte elongation. Since this correlation has interesting implications for co-regulation of shape and gene expression, we decided to investigate this relationship further. CITED4 is upregulated with exercise and associated with myocyte proliferation [28], making it a potential therapeutic target for cardiac regeneration. Moreover, eccentric hypertrophy due to myocyte elongation is associated with severe cardiac dysfunction [31], making signaling regulating myocyte elongation an especially important area for further investigation. While elongation/CITED4 correlation could arise from a shared upstream pathway without causal relationships between CITED4 and elongation, we hypothesized that CITED4 mechanistically regulated elongation in cardiac myocytes.

To test this hypothesis, we knocked down CITED4 with siRNA (Figure 4.27A) and measured the effects on Nrg1 and LIF-induced elongation of cardiac myocytes. Nrg1 and LIF were chosen since they 
both induce myocyte elongation, but had different effects on CITED4 mRNA abundance (strong upregulation vs. no effect respectively, see Figure 4.11). CITED4 knockdown did not affect elongation in control myocytes and significantly increased Nrg1-induced elongation (Figure 4.27B-C). These data are both consistent with Nrg1-induced elongation and upregulation of CITED4 as measured in the agonist screen (Figure 4.6) and indicate that CITED4 negative regulates myocyte elongation, forming an incoherent feedforward loop [88]. Moreover, while LIF did not specifically upregulate CITED4 expression, CITED4 knockdown below basal levels also increased elongation in LIF treated myocytes.

To test whether the proposed incoherent feed-forward loop can quantitatively explain the measured relationships between CITED4 mRNA abundance and myocyte elongation, we developed a minimal mathematical model using ordinary differential equations (Figure 4.27D). Equations and a detailed schematic of the model are provided in Appendix E. In the model, Nrg1 stimulates myocyte elongation and CITED4 expression, LIF stimulates myocyte elongation, and CITED4 expression negatively regulates myocyte elongation. These processes are modeled using saturating, Michaelis-Menten form kinetics, with CITED4 negative regulation of elongation only occuring if the myocytes have increased elongation compared to control (fold change in elongation > 1). CITED4 mRNA and elongation are also influenced by constant basal production and linear degradation terms.

We fit the model parameters to CITED4 and elongation data from control siRNA experiments using nonlinear least-squares fitting (Figure 4.27D) and then independently validated the predicted the change in myocyte elongation given the experimentally determined amount of CITED4 knockdown (Figure 4.27E). Note that the model predictions of elongation after CITED4 knockdown closely matched the elongation data, even though the model was not fit to this condition (Figure 4.27E). We next 

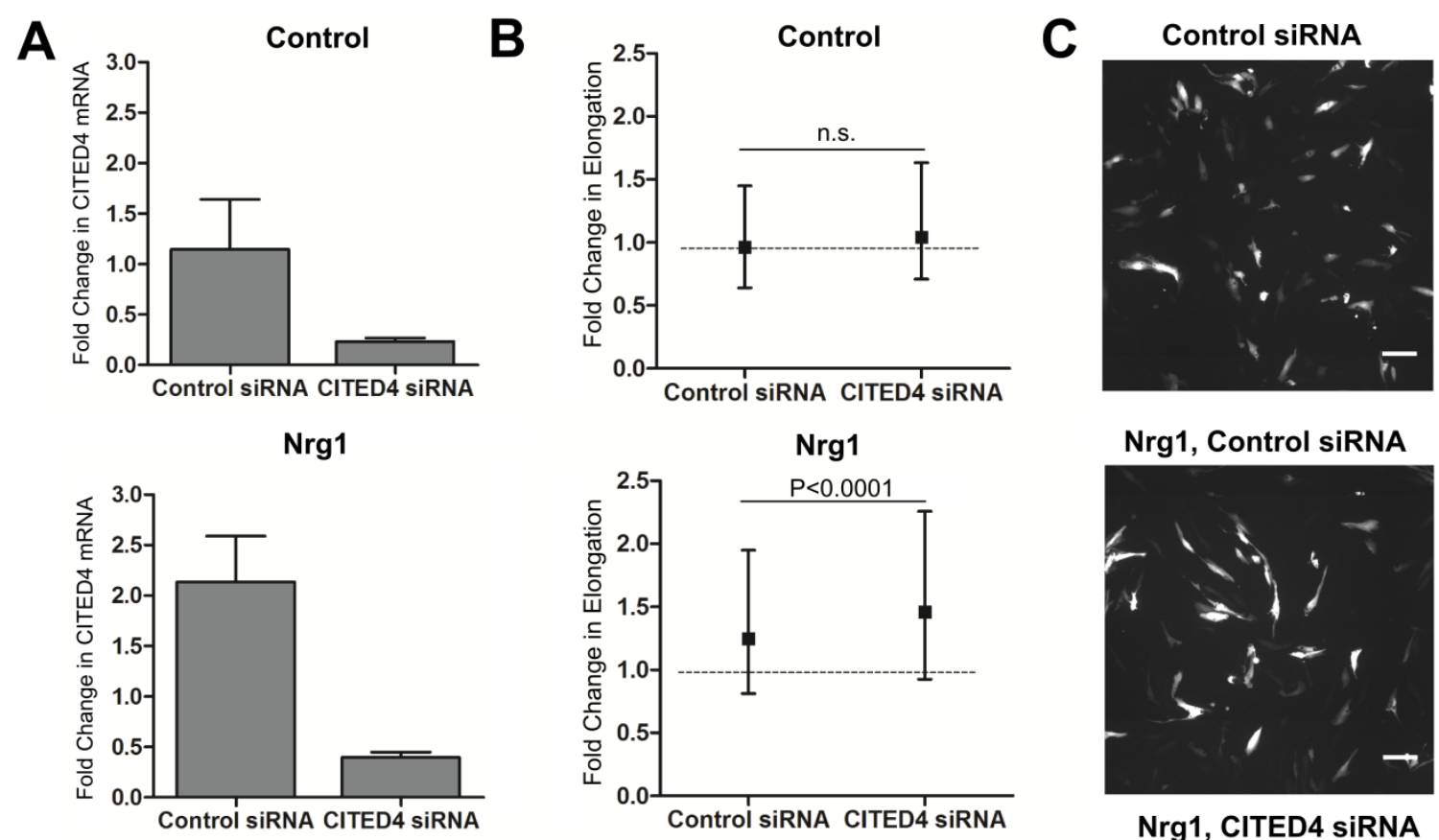

Nrg1, Control siRNA
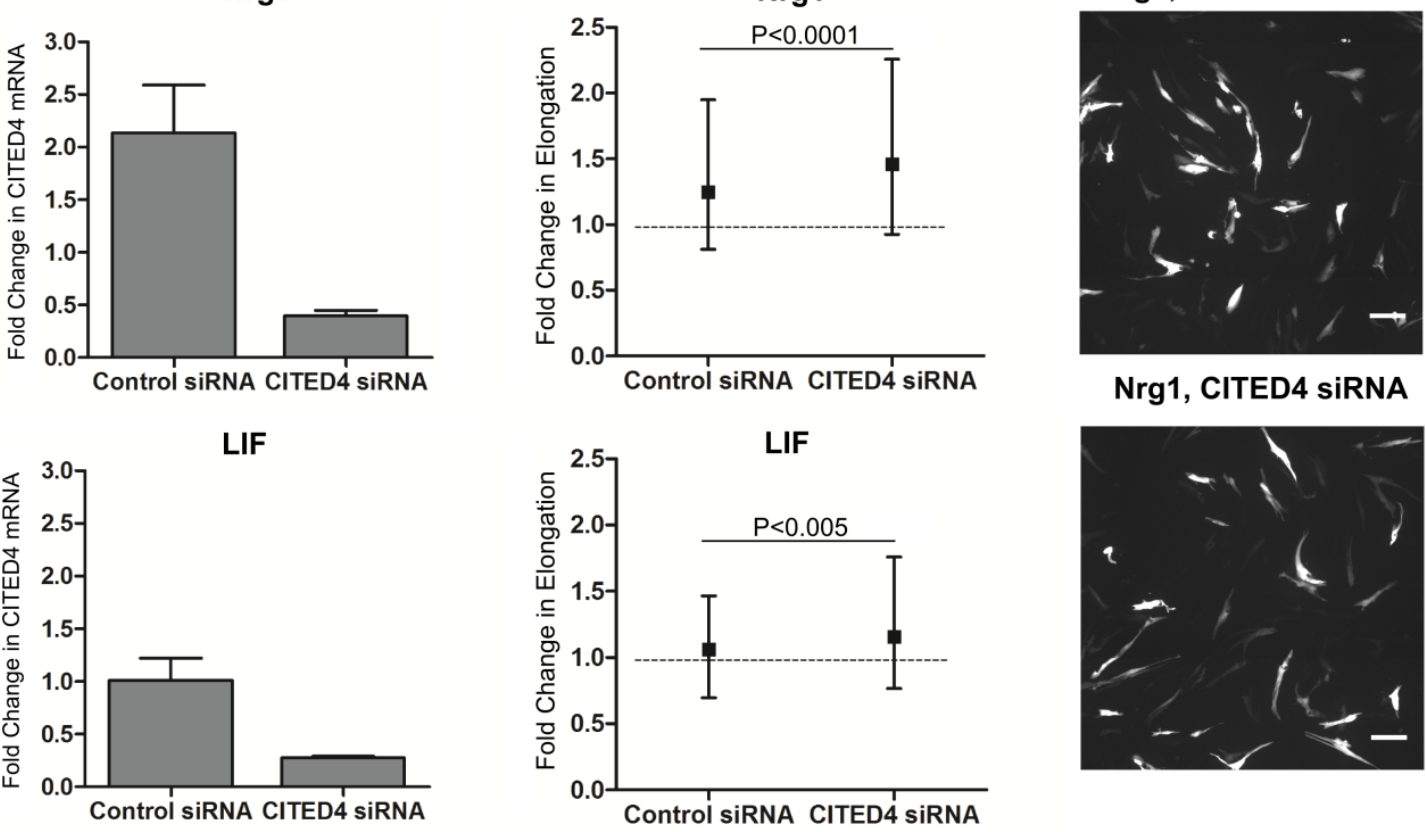

Nrg1, CITED4 siRNA

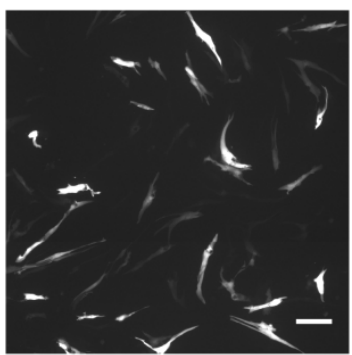

\section{Control siRNA}

D

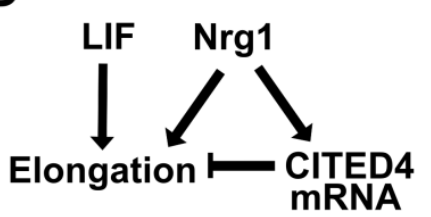

E

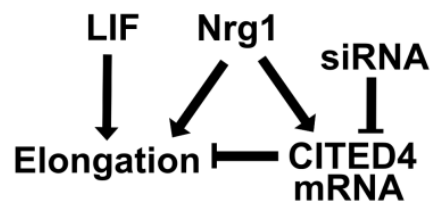

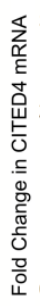
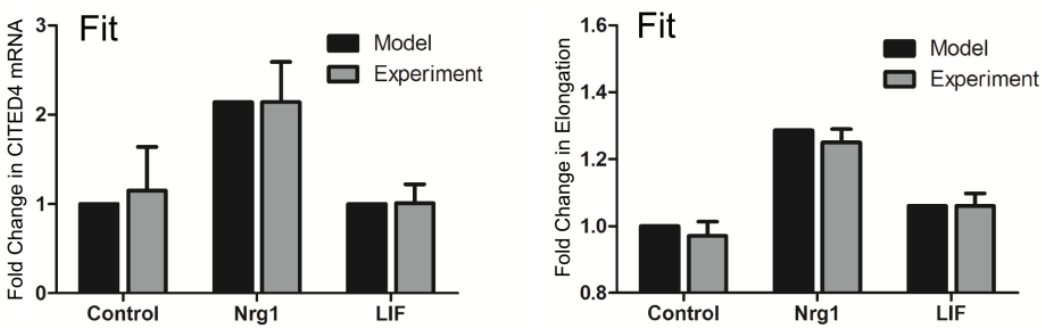

CITED4 SIRNA
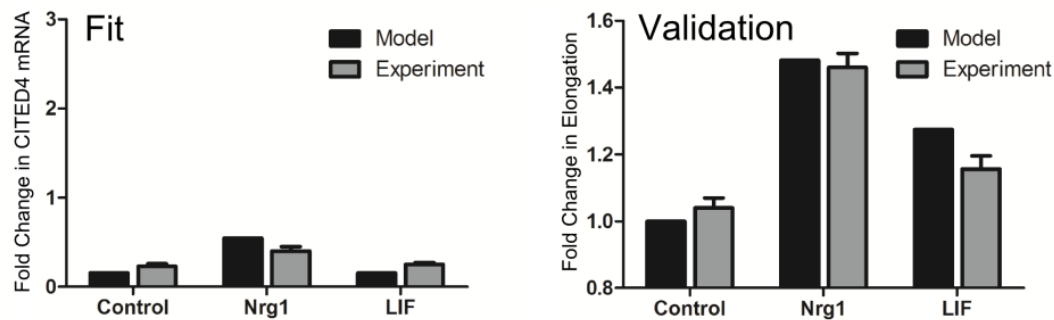


\section{Figure 4.27: CITED4 knockdown enhances Nrg1 and LIF-induced myocyte elongation via an incoherent}

feed-forward loop. A) Mean +/- SE of CITED4 mRNA abundance. CITED4 mRNA was knocked down by siRNA transfection. CITED4 mRNA levels were quantified using qPCR. Myocytes were treated with control, $10 \mathrm{ng} / \mathrm{mL} \mathrm{Nrg1}$, or $1 \mathrm{nM}$ LIF for 48 hours after transfection with control or CITED4 siRNA. B) Median and interquartile range of fold change in myocyte elongation for control, Nrg1, and LIF treated myocytes. Nrg1 and LIF-treated myocytes significantly increased elongation after CITED4 knockdown (two-tailed Mann Whitney $\mathrm{U}$ test, $\mathrm{N}^{\sim 700}$ myocytes), indicating that CITED4 negatively regulates elongation. C) Representative images of control myocytes and Nrg1-treated myocytes with control siRNA or CITED4 siRNA (Scale bar: $100 \mu \mathrm{m}$ ). CITED4 knockdown enhanced Nrg1-induced myocyte elongation. D) Minimal mathematical model of CITED4 regulation of myocyte elongation, fit to CITED4 mRNA and elongation data in control siRNA conditions. E) Incorporating measured levels of CITED4 mRNA knockdown, the model accurately predicted increased Nrg1- and LIF-dependent myocyte elongation in CITED4 siRNA conditions (an independent experimental validation). 
explored whether alternative simpler models may also be able to predict the data. We calculated the sum of squared errors of prediction (SSE) to quantitatively compare the appropriateness of selecting one model over another, where a smaller SSE indicates a higher predictive ability. We found that negative regulation of elongation by CITED4 (Figure 4.28) and a minimum elongation threshold for negative regulation by CITED4 (Figure 4.29) were necessary for our model predictions of elongation to validate against our independent experimental observations with CITED4 siRNA. Indeed, model variants lacking CITED4 regulation of elongation $(\mathrm{SSE}=0.055)$ or the elongation threshold $(\mathrm{SSE}=0.029)$ performed worse in experimental validation tests than the default model $(\mathrm{SSE}=0.016)$. Our experimental and modeling results imply that CITED4 mRNA abundance and myocyte elongation were correlated in our hypertrophic agonist screen due to coincident regulation by Nrg1. Moreover, Nrg1, CITED4, and myocyte elongation form an incoherent feedforward loop where CITED4 negatively regulates elongation. 

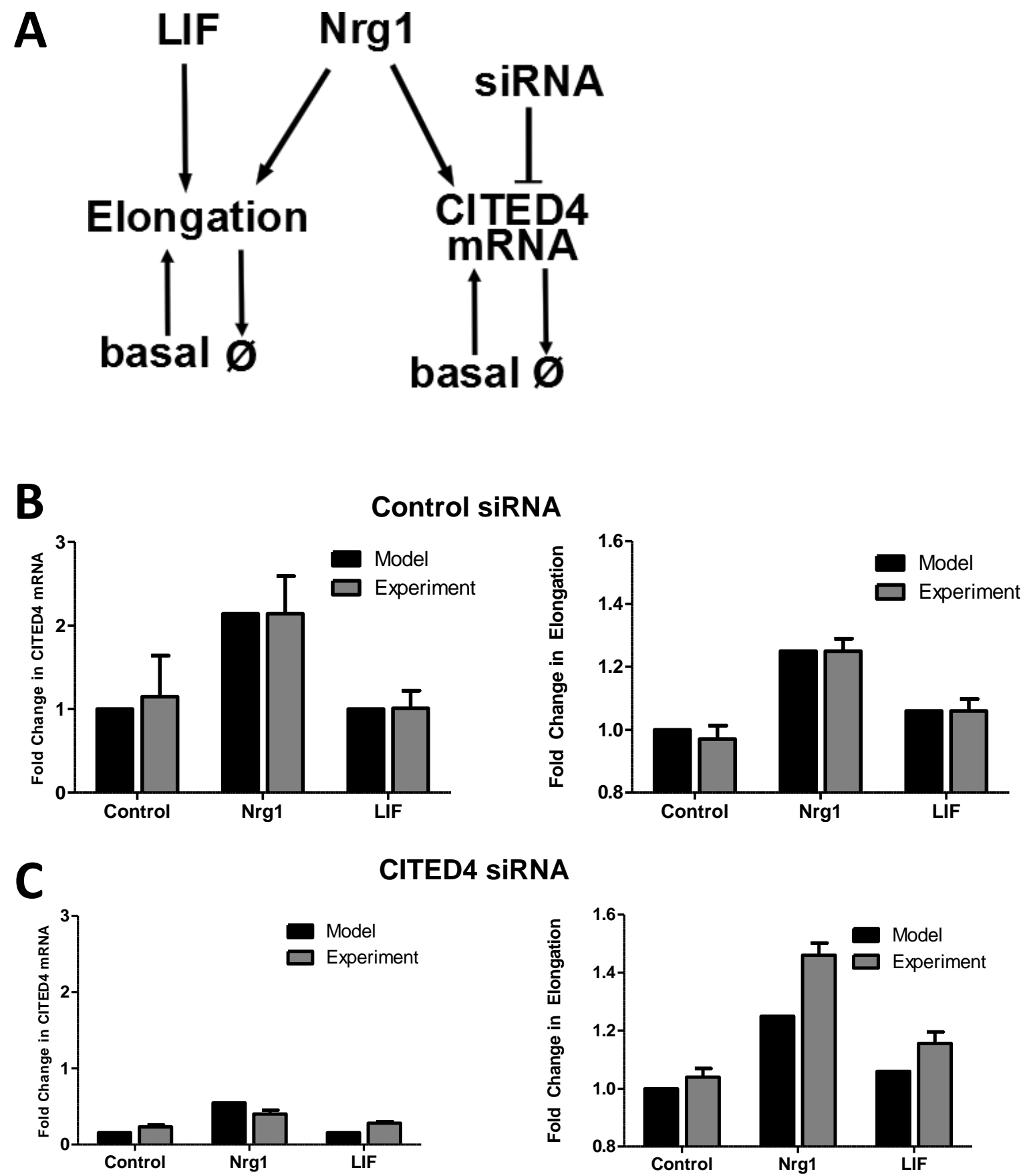

Figure 4.28: Computational model lacking CITED4 regulation of myocyte elongation cannot predict enhanced myocyte elongation after CITED4 knockdown. A) Schematic of an ordinary differential equation model of LIF, Nrg1, CITED4, and Elongation with no link between CITED4 mRNA and myocyte elongation. In the model, Nrg1 stimulates myocyte elongation and CITED4 expression and LIF stimulates myocyte elongation. These processes are modeled using saturating, Michaelis-Menten form kinetics. CITED4 mRNA abundance and elongation are also influenced by constant basal production and linear degradation terms. B) We fit the model to CITED4 mRNA and elongation data with control siRNA. C) 
After fitting to the measured level of CITED4 mRNA knockdown, the model independently predicted myocyte elongation with CITED4 siRNA. This model structure is not able to predict enhanced Nrg1 and LIF-induced elongation with CITED4 siRNA knockdown.

\section{Control siRNA}
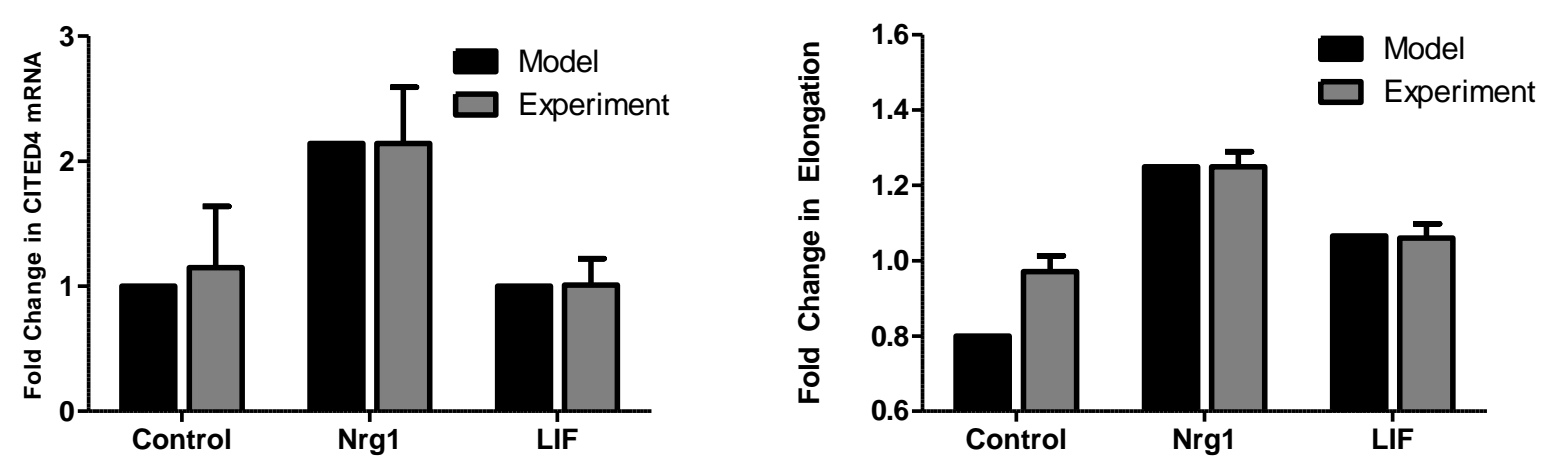

CITED4 siRNA
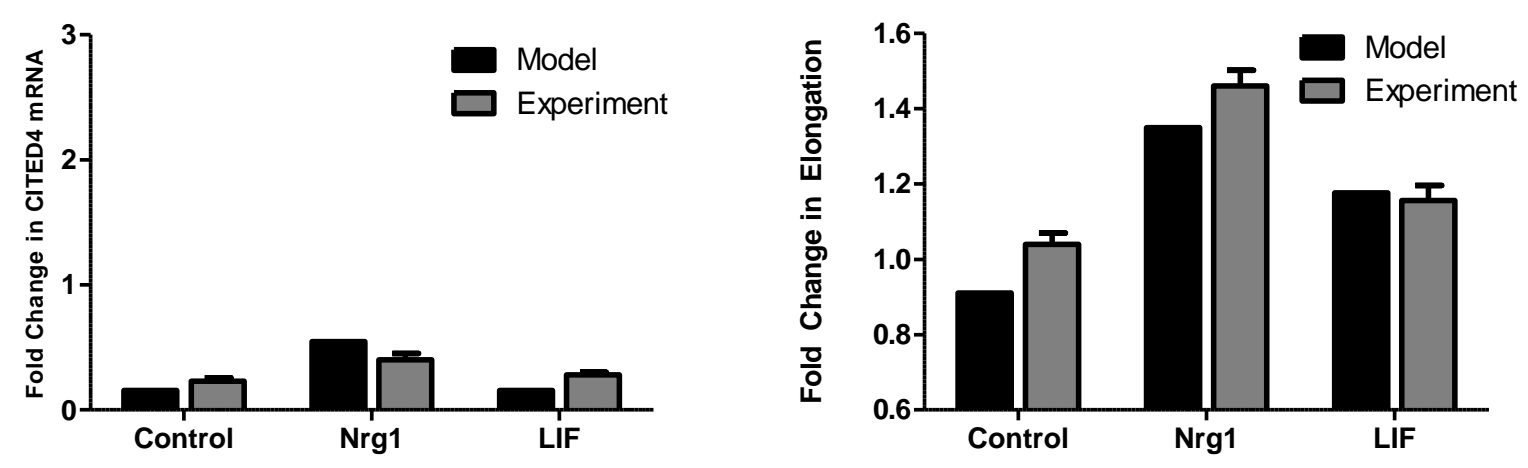

Figure 4.29: Computational model lacking minimum myocyte elongation value for CITED4 regulation of myocyte elongation cannot predict control CITED4 siRNA data. We developed a version of our Nrg1, LIF, CITED4, and elongation model where there was no longer a minimum elongation value for the myocytes. Therefore, CITED4 mRNA could negatively regulate myocyte elongation at any value of elongation. A) We fit the model to CITED4 mRNA and elongation data with control siRNA. B) After fitting to the measured level of CITED4 mRNA knockdown, the model independently predicted myocyte elongation with CITED4 siRNA. This model structure produces a worse fit to our data compared to Figure 7D (with a minimum elongation value) and predicts increased elongation for control cells with CITED4 knockdown, which is not consistent with our experimental observations. This model structure therefore makes less accurate predictions of myocyte elongation after CITED4 knockdown. 


\subsubsection{CITED4 overexpression increases cardiac myocyte size and myocyte proliferation}

Our minimal model of CITED4 and elongation required incorporating an elongation threshold above which CITED4 negatively regulated elongation (Figure 4.29). This was initially validated in our CITED4 siRNA experiments where CITED4 knockdown further enhanced elongation in Nrg1 and LIF treated myocytes, but not with control myocytes (Figure 4.27A-C). As a corollary, our model predicts that overexpression of CITED4 does not affect elongation of unstimulated cardiac myocytes (Figure 4.30). To test this hypothesis, we treated neonatal rat cardiac myocytes with adenoviral constructs expressing CITED4, or lacZ as a control. As predicted by the model, increased expression of CITED4 did not significantly affect myocyte elongation (Figure 4.30A-B). However, overexpression of CITED4 did increase the average cell area, fraction of myocytes expressing proliferation markers Ki67 and EdU, and myocyte number (Figure 4.30C-F), demonstrating activity of the construct. Therefore, CITED4 overexpression induces myocyte proliferation, as previously reported [28], and myocyte hypertrophy consistent with the phenotype of physiological hypertrophy. 


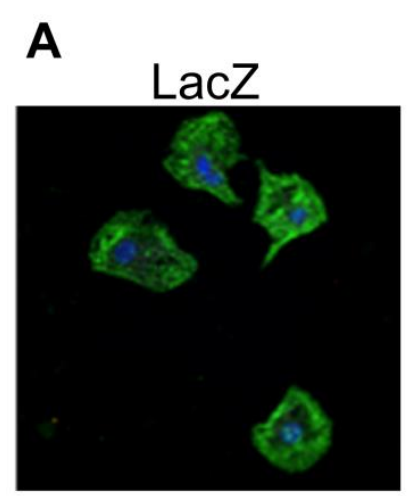

CITED4

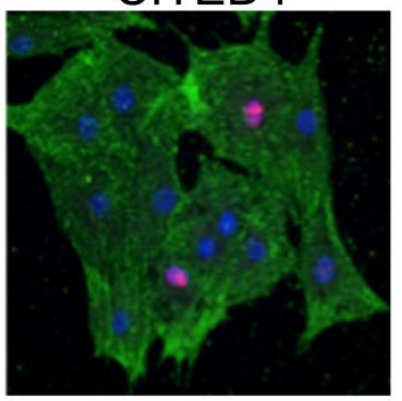

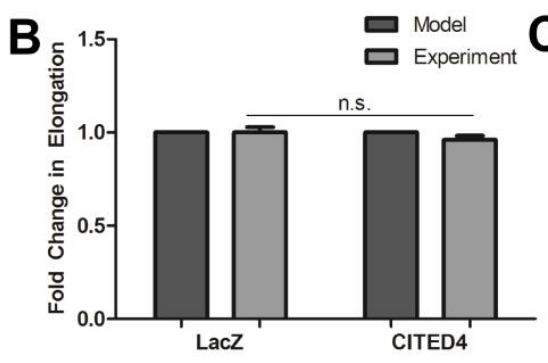
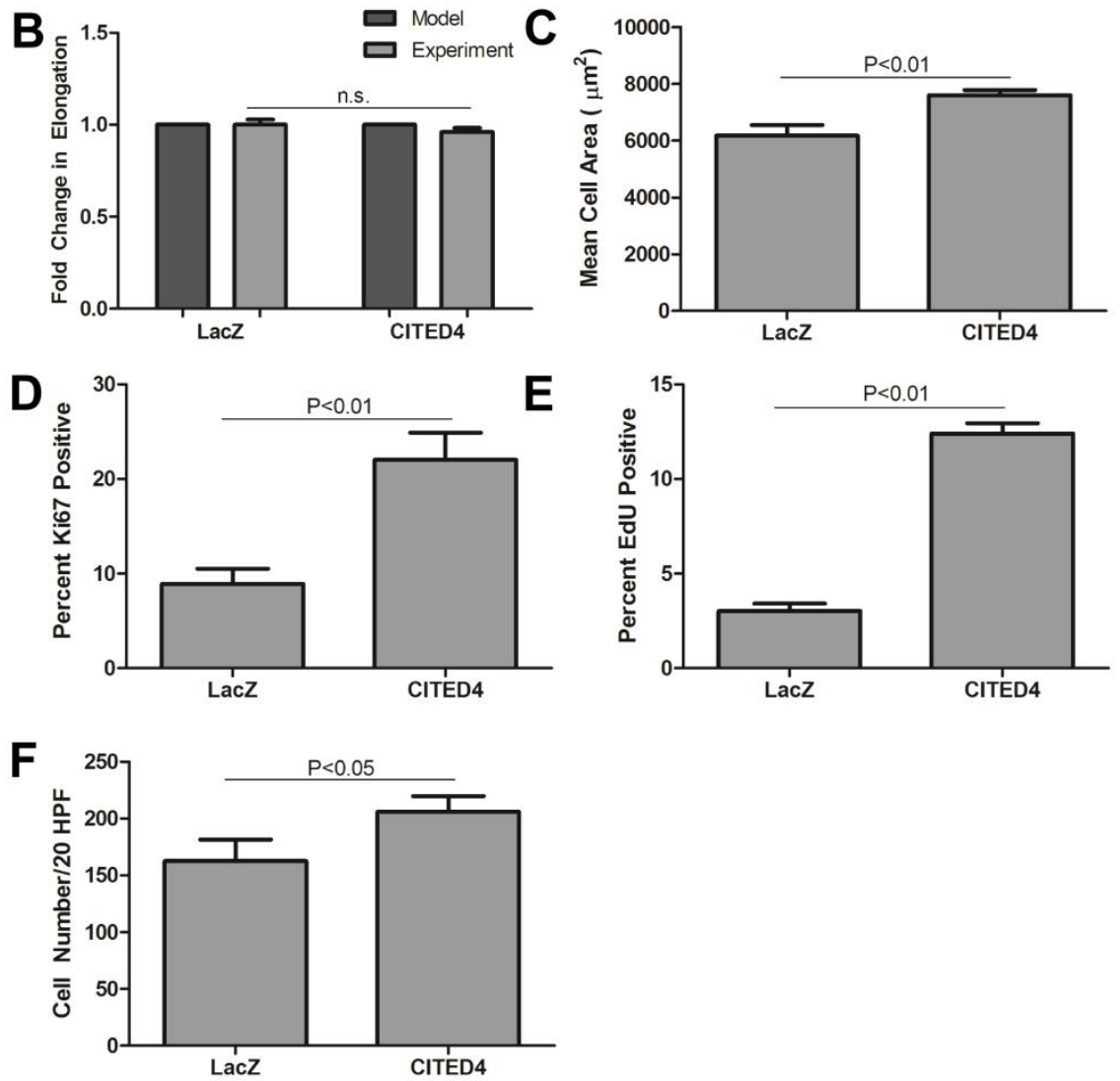

Figure 4.30: Overexpression of CITED4 induces myocyte proliferation and hypertrophy without affecting elongation. Neonatal rat ventricular myocytes were treated with either CITED4-expressing adenovirus or lacZ control. Experiments were performed 24 hours after infection. Data was collected from 3 independent experiments with 500 myocytes per condition, reported as mean +/- SE. A) Representative images of lacZ and CITED4-expressing myocytes labeled for theproliferation marker Ki67 (red), $\alpha$-actinin (green), and DAPI (blue). B) Model prediction and independent experimental validation of change in myocyte elongation after CITED4 overexpression. CITED4 overexpression without an elongation stimulus such as Nrg1 or LIF did not affect myocyte elongation, as predicted by our model. C) Myocyte area, D) percentage of myocytes positive for proliferation markers Ki67 and E) EdU, F) and quantification of cardiac myocyte cell number. HPF indicates high-power field. CITED4 overexpression induced increases in myocyte area and myocyte proliferation without affecting elongation.

Experimental data generated by collaborators Vassilios J. Bezzerides (Department of Cardiology, Children's Hospital Boston, Harvard Medical School) and Anthony Rosenzweig (Cardiovascular Division of the Beth Israel Deaconess Medical Center, Harvard Medical School). 


\subsection{Discussion}

Quantitative understanding of the distinct contributions of signaling pathways to specific changes in shape and mRNA expression is needed to better understand and ultimately control the molecular circuits governing cardiac hypertrophy. Here, we performed a screen of 15 hypertrophic agonists in neonatal rat ventricular myocytes and quantified differential regulation of 5 shape features and transcript abundance of 12 genes. While cultured myocytes do not fully reproduce the complexity of the heart, they are essential for scalable high-throughput studies, and neonatal myocytes are the best characterized cellular system for studying myocyte hypertrophy [126]. We plated myocytes on SureCoat for optimal cell health, but consideration of other substrates would be interesting in future work, particularly in studies of mechanotransduction pathways such as integrin signaling.

Previous work has established a role for these 15 agonists in hypertrophy [13], [107], but the contributions of these pathways to distinct hypertrophic features and relative dominance among the agonists to specific phenotypic outputs was less characterized. We previously compared changes in shape and sarcomere organization between four of the hypertrophic agonists, PE, Iso, IGF1, and TNF $\alpha$ [37]. We extended this work to measure transcript abundance in addition to shape with a more diverse panel of agonists. This allowed us to better characterize differential regulation of maladaptive and adaptive hypertrophy features and identify transcripts regulating Bax mRNA and myocyte elongation.

Previous work has demonstrated systems analysis of a single ligand screen in other cell types [127] and high-content screening of cardiac myocyte size in fixed cells after perturbation with microRNA libraries [38]. Here, we used live cell imaging of cardiac myocytes to measure fold-changes in 5 shape features. Live-cell imaging using plasmid-based transfection allows for isolation of RNA from the same myocytes we used to measure shape changes, conspicuous cell boundaries suitable for automated 
segmentation from monolayers of myocytes, and measurement of fold-changes in shape of individual myocytes, which has less dispersion than raw data without tracking [39].

While many commonly measured markers of hypertrophy such as cell size and fetal gene expression are not substantially differentially regulated between hypertrophy pathways [107], measuring outputs related to shape, fibrosis, cell death, inflammation, and proliferation revealed distinct phenotypic signatures among the hypertrophy pathways. Correlation coefficients among the shape features further reveal differential regulation of cell area, elongation, and form factor. Distinct regulation of elongation is of substantial interest due to the in vivo significance of concentric and eccentric hypertrophy [101]. We used our data of differential regulation of outputs to identify modules within inputs and outputs. These modules revealed shared regulation of many maladaptive features such as cell death, fibrosis, and inflammation. Shared regulation of maladaptive hypertrophy features could provide insight into the observation of distinct in vivo presentations of pathological and physiological hypertrophy [17]. Additionally, protective outputs VEGF and Bcl2 were in a module with measures of cell size and hypertrophy (fetal gene program). VEGF is important for adaptive growth of the heart [114], [128], and $\mathrm{Bcl} 2$ would further protect the heart during growth [129], [130]. While we examined cell size, shape and mRNA at 48 hours, additional causal relationships may be revealed by examining earlier time points.

Hierarchical clustering of the ligand screen data revealed correlation between mRNA abundance of pro-fibrotic CTGF and pro-cell death Bax. Correlation between these two features is interesting given association with cardiac myocyte death and dilated cardiomyopathy and heart failure [131], [132] and association of fibrosis with diastolic dysfunction and arrhythmias [133], [134]. Previous work has shown that CTGF stimulates proliferation in fibroblasts and endothelial cells [135] but apoptosis in human breast cancer cells[136] and human aortic smooth muscle cells [137], [138]. CTGF protein levels are 
increased in both the infarct region and viable myocardium after myocardial infarction in rats indicating involvement by CTGF in early and late stage cardiac remodeling [139]. Our follow-up experiments showed that increased CTGF was sufficient to increase Bax mRNA abundance and that knocking down CTGF decreased Ang II-dependent increases in Bax mRNA. Pathological hypertrophy is associated with increased fibrosis and cell death and shared regulation between CTGF and Bax could accelerate development of cardiac dysfunction. Therapies for hypertrophy that decrease CTGF could therefore provide some protection from cell death in addition to protection from fibrosis.

We identified an incoherent feed forward loop for Nrg-1-induced elongation where Nrg-1 increased myocyte elongation and CITED4 mRNA abundance, while CITED4 mRNA negatively regulates myocyte elongation. Further study of the dynamics of changes in myocyte elongation and CITED4 mRNA abundance will be needed to fully explore the biological significance of this type 1 incoherent feed forward loop. Previous studies of this signaling motif in other systems have shown that incoherent feed forward loops can induce pulses in signaling and accelerate signaling response time [88], [140]. Previous work has also shown Nrg1 and LIF-induced myocyte elongation [141]. Nrg1 also induced the largest increases in CITED4 mRNA, which has been previously shown to induce myocyte proliferation [28]. Therapeutic importance of Nrg1 signaling has been shown in previous mouse studies. Knockout of Erbb4, a member of the Nrg1 signaling pathway, led to dilated cardiomyopathy and premature death in mice [142]. Moreover, administration of Nrg1 to rats with cardiac dysfunction reduced hypertrophy and increased fractional shortening and ejection fraction [143].

While Nrg1 induced myocyte elongation at 48 hours, coincident increases in CITED4 mRNA worked to attenuate elongation. Since eccentric hypertrophy has been linked to higher risk for systolic dysfunction and heart failure[31], [144], there has been great interest in learning about specific signaling pathways that regulate myocyte elongation [30], [145]. Increased CITED4 may therefore be beneficial to 
the heart by preventing high levels of myocyte elongation in addition to its previously studied role in exercise induced myocyte proliferation. However, it will be important in future studies to determine CITED4's role in regulation of adaptive phases of eccentric hypertrophy versus maladaptive phases that lead to heart failure [106], [146]. Moreover, we showed that overexpression of CITED4 increased myocyte area and proliferation. While CITED4 and area were not as highly correlated as CITED4 and elongation, we did observe high CITED4 mRNA levels with ET1, PE, and NE, the agonists causing the highest increases in cell area.

Conversely, LIF increased myocyte elongation without increased CITED4 mRNA to buffer elongation, making it a potentially more deleterious inducer of elongation. CITED4 knockdown below basal levels still enhanced LIF-induced elongation, indicating basal CITED4 expression in the myocytes that protects against elongation signaling. Previous work has demonstrated the importance of ERK5 signaling in LIF-induced elongation [147]. LIF also significantly increased abundance of C/EBP $\beta$ mRNA, a gene down-regulated in exercise-induced hypertrophy [28]. Moreover, while skeletal $\alpha$-actin and BNP were similarly regulated, LIF selectively increased BNP and not skeletal $\alpha$-actin. High BNP levels have been associated with increased cardiovascular morbidity and mortality [148], [149]. These findings indicate LIF signaling may be especially detrimental in pathological hypertrophy.

In summary, by measuring 5 shape features and 12 mRNAs we quantified distinct phenotypic signatures for 15 major hypertrophic agonists. This result is significant given the high level of cross talk in the hypertrophy signaling network and lack of differential regulation between pathways in commonly measured hypertrophy readouts such as fetal gene expression [107]. Follow-up experiments identified positive regulation of Bax mRNA abundance by CTGF, negative regulation of myocyte elongation by CITED4, and increased cell size with CITED4 overexpression. 
Chapter 5

\section{Reverse Phase Protein Array proteomics analysis of hypertrophic agonists}




\subsection{Introduction}

In Chapter 4 we measured differential regulation of myocyte shape and gene expression in response to different hypertrophic agonists. Moreover, while hypertrophy pathways are often depicted as being independent, our model analysis in Chapter 3 revealed a new conceptual model where receptors act in a crosstalk dense network, with many signals funneling through two network hubs: PI3K and Ras. Therefore Ras and PI3K may be key decision points in the network for these different hypertrophy features. Indeed, our model predicted Ras to be the most influential protein in the hypertrophy signaling network. Moreover, Ras and PI3K overexpression result in unique phenotypes in vivo. Ras overexpression induced pathological hypertrophy[150], [151] and PI3K expression is important for physiological hypertrophy[25]. Therefore we hypothesize that the balance of signaling out of PI3K and Ras determines the form of myocyte hypertrophy. We hypothesize that high Ras signaling relative to PI3K results in more pathological features such as increased elongation, fibrosis, cell death, and that high PI3K signaling relative to Ras results in more protective hypertrophic features such as proliferation, growth without elongation, protection from cell death, and myocyte proliferation. Here, we stimulate myocytes with 5 different hypertrophic agonists and measure the magnitude of Ras and PI3K signaling. In future studies, we will measure changes in myocyte shape and gene expression after inhibiting PI3K, Ras, or both.

\subsection{Methods}

To measure PI3K and Ras signaling, we used Reverse Phase Protein Array (RPPA) analysis to measure changes in protein levels of 170 different proteins. RPPA is a high-throughput antibody-based method for measuring protein concentration similar to Western blots. While PI3K and Ras activity were not directly measured using this method, we were able to measure the phosphorylation state of many downstream species such as AKT, GSK3 $\beta$, mTor, cRaf, cJun, MEK1, p38, MAPK, and JNK. 
Neonatal rat ventricular myocytes were cultured in 24 -well plates $(500,000$ myocytes/well) and

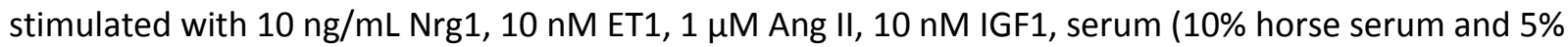
fetal bovine serum), or control. Myocyte protein was isolated at two time points: 1 hour and 48 hours according to the protocol on the MD Anderson Cancer Center RPPA Core Facility website. Protein concentration was quantified using the Pierce 660 nM Protein Assay Kit (Thermo Scientific). Cell lysates were submitted to the MD Anderson Cancer Center for analysis. Data was collected from 2 independent myocyte isolations per condition. All data points were normalized for protein loading.

\subsection{Results}

For each condition, we rank ordered the proteins with the greatest fold change compared to control. Below, we display bar graphs showing the top $10 \%$ of proteins with the greatest fold changes. We observed many proteins in MAP Kinase and AKT signaling pathways in these top $10 \%$ lists and also many species related to cell growth signaling (e.g. S6, p70S6 Kinase, and YAP1). ET1 induced large increases in the most MAPK species. Phosphorylated MAPK, cRaf, MEK1, and p38 were high at 1 hour and phosphorylated AKT was high 48 hours (Figure 5.1). Ang II's list of highly upregulated proteins includes much less MAPK and AKT related species. At 1 hour only phosphorylated MAPK is high. Although notably at 48 hours, cell death related proteins Bax and cleaved caspase 7 are high with Ang II (Figure 5.2). Nrg1 exhibited high changes in phosphorylated AKT, GSK3, and MAPK at both time points (Figure 5.3). Serum showed larger increases in phosphorylated MAPK at 1 hour and AKT and GSK3 at 48 hours (Figure 5.4). Finally, IGF showed larger increases in phosphorylated MAPK at 1 hour (Figure 5.5). Other signaling proteins not in the MAPK or AKT pathways that were commonly seen in the lists of proteins with the greatest changes were phosphorylated STAT3 and PKC. 
A.

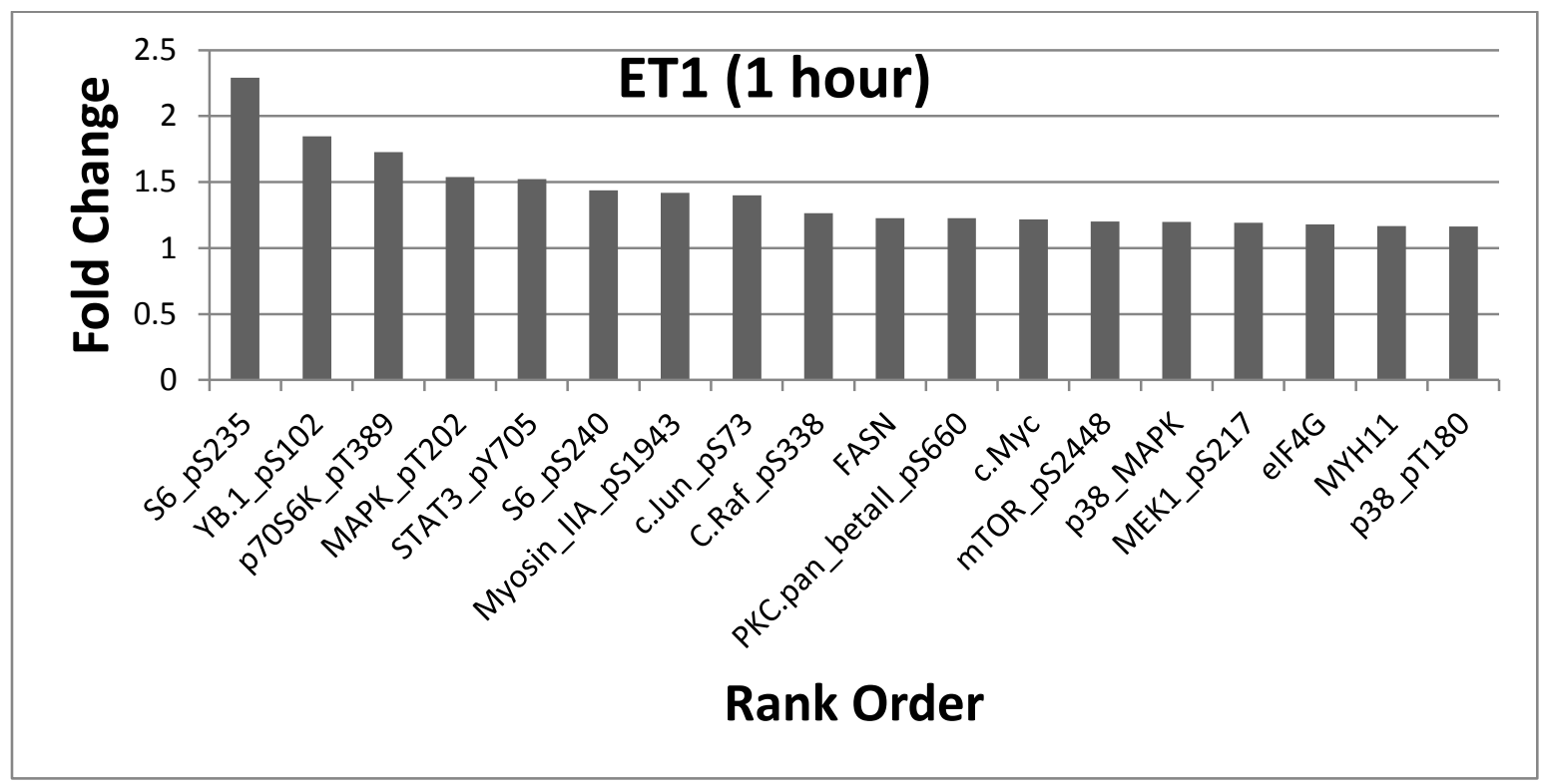

B.

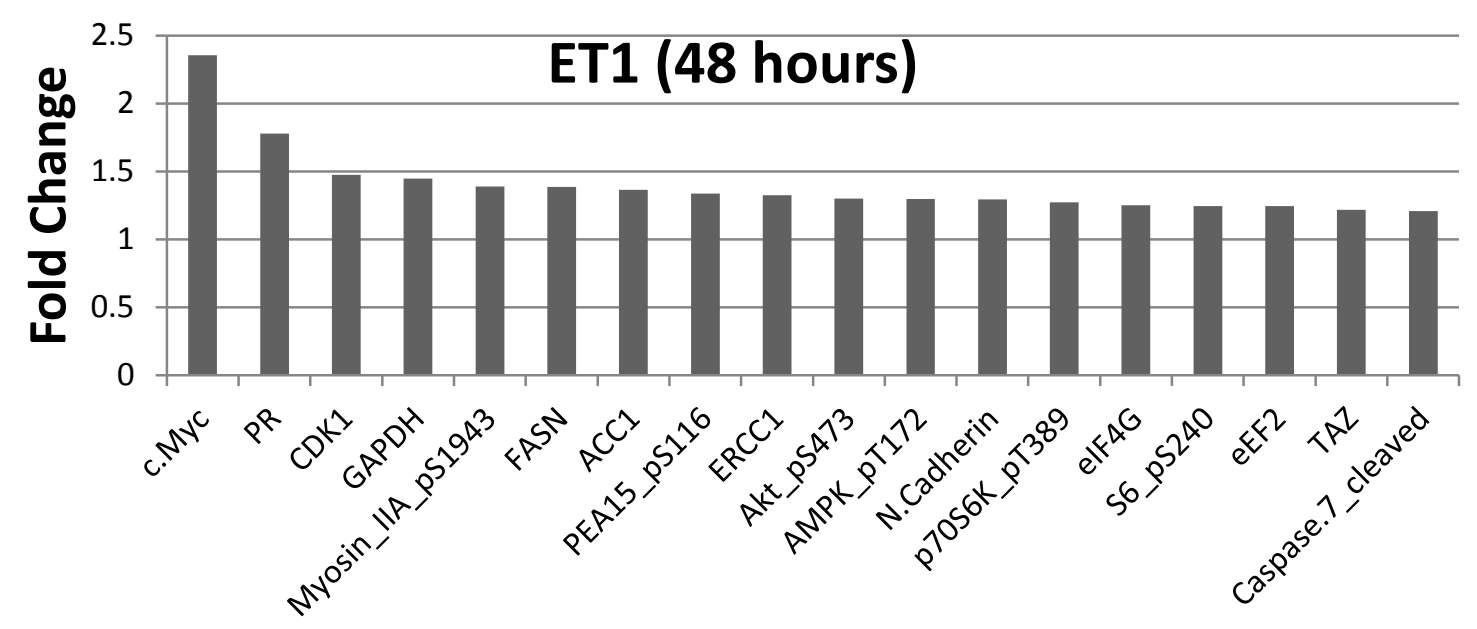

Rank Order

Figure 5.1 Rank ordered list of average fold change in protein concentration compared to control for duplicate samples treated with 10 nM ET1 for A) 1 hour and B) 48 hours. 
A.

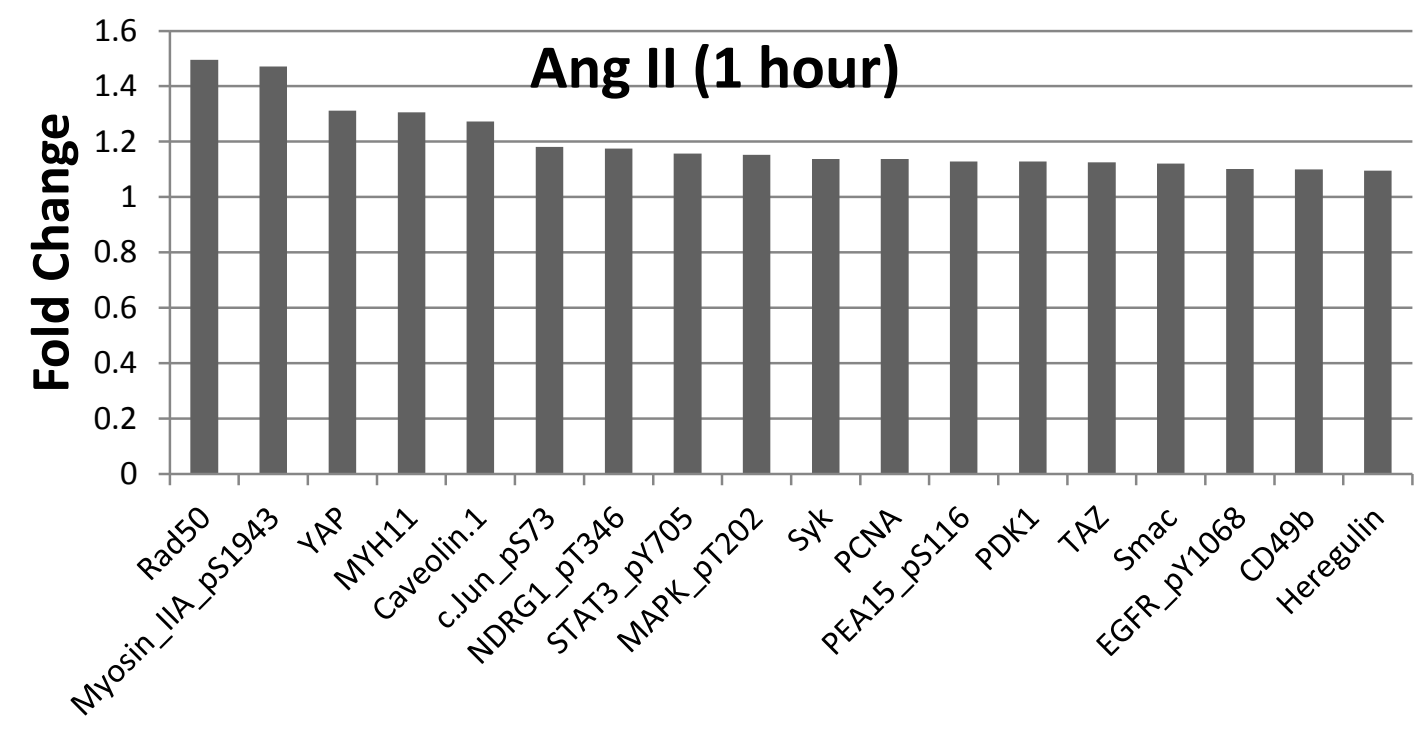

Rank Order

B.

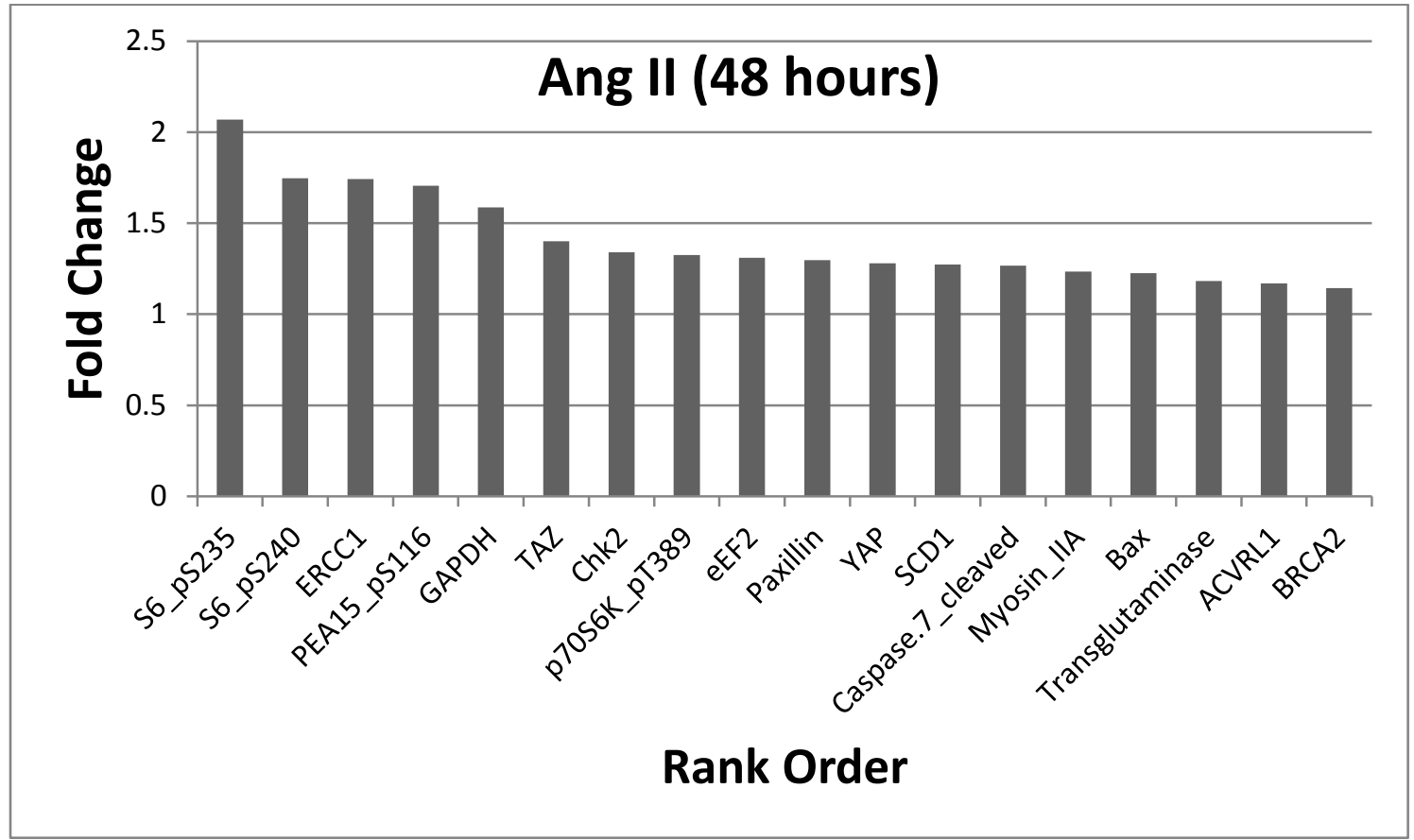

Figure 5.2 Rank ordered list of average fold change in protein concentration compared to control for duplicate samples treated with $1 \mu \mathrm{M}$ Ang II for A) 1 hour and B) 48 hours. 
A.

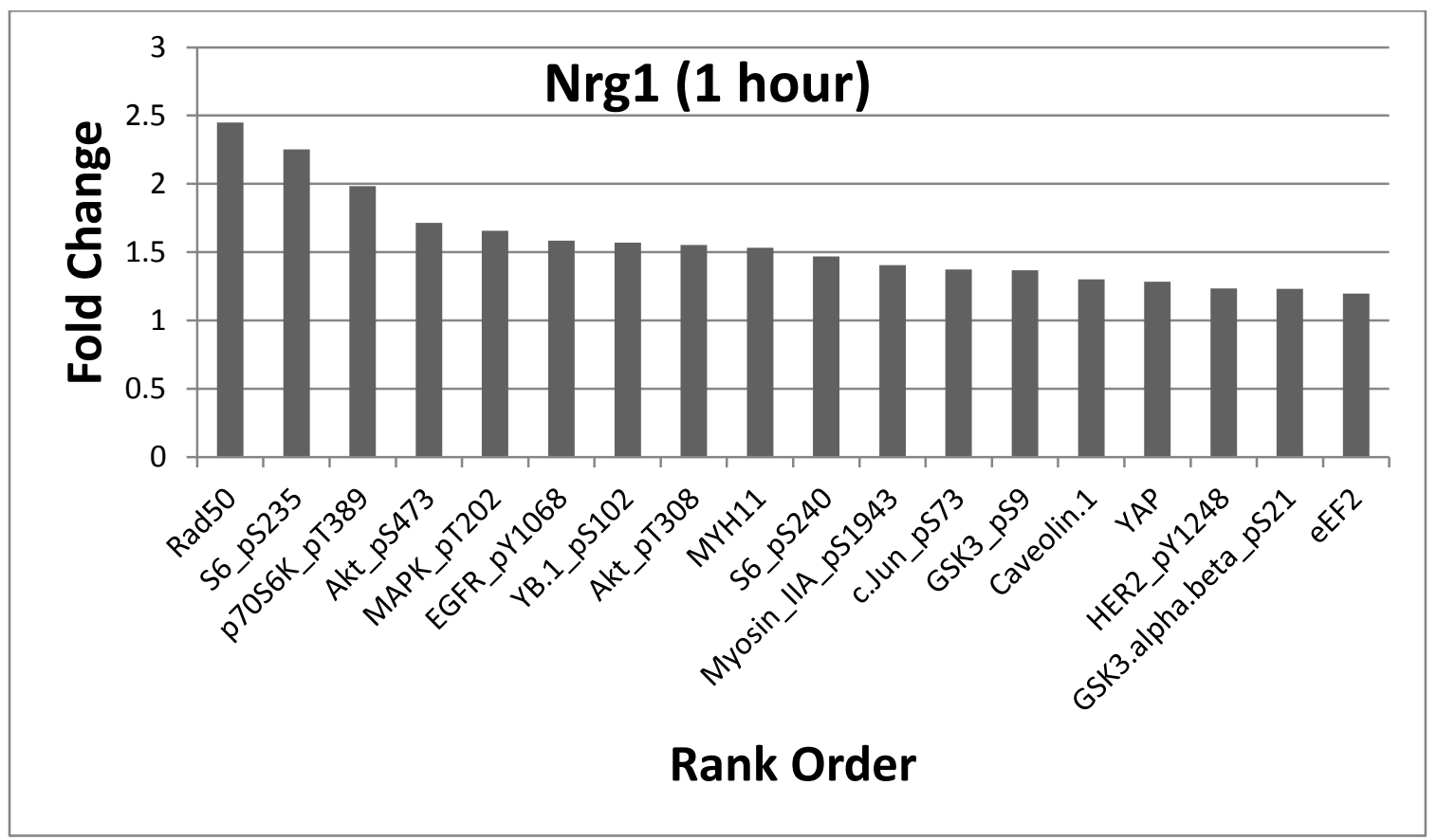

B.

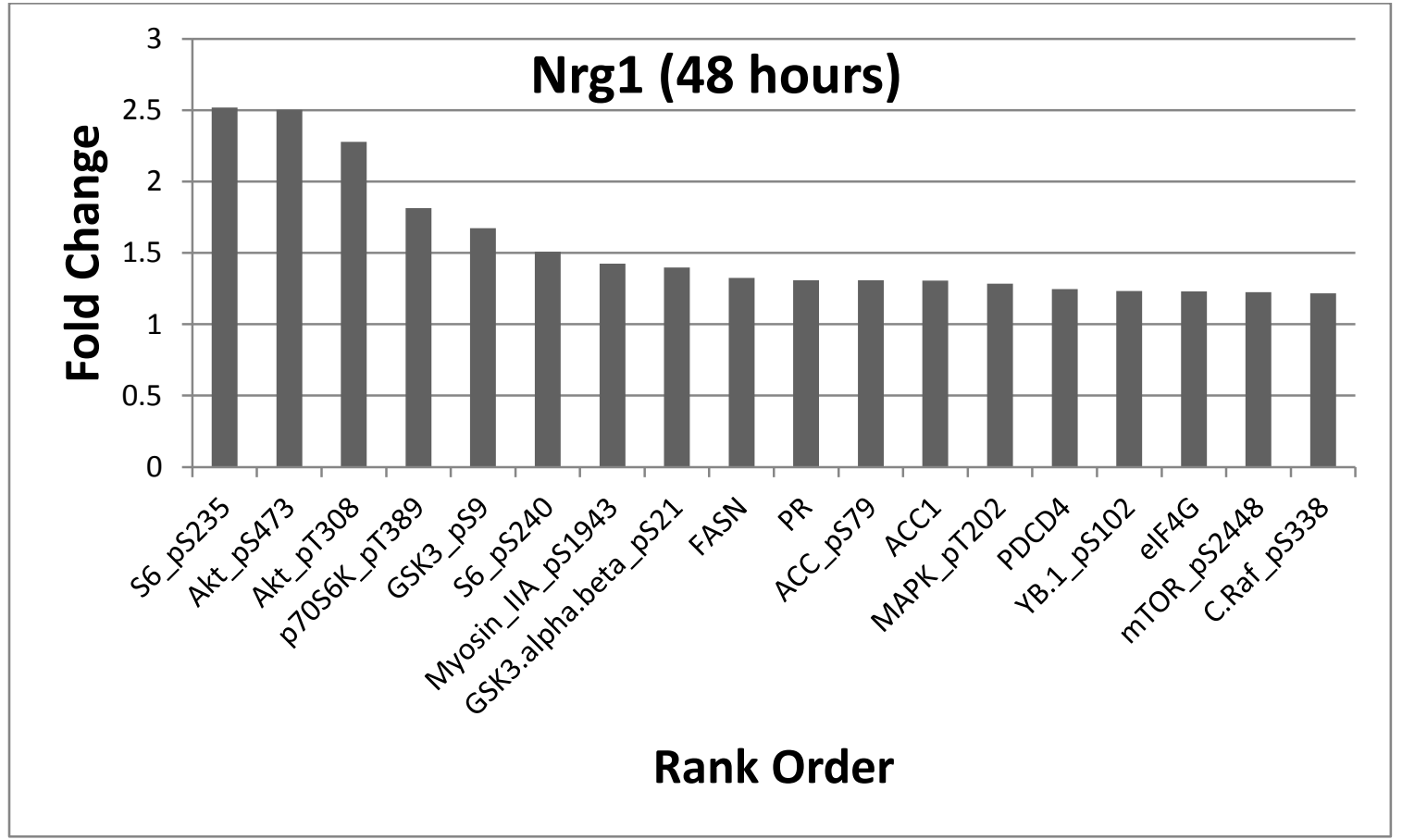

Figure 5.3 Rank ordered list of average fold change in protein concentration compared to control for duplicate samples treated with $10 \mathrm{ng} / \mathrm{mL} \mathrm{Nrg1}$ for A) 1 hour and B) 48 hours. 
A.

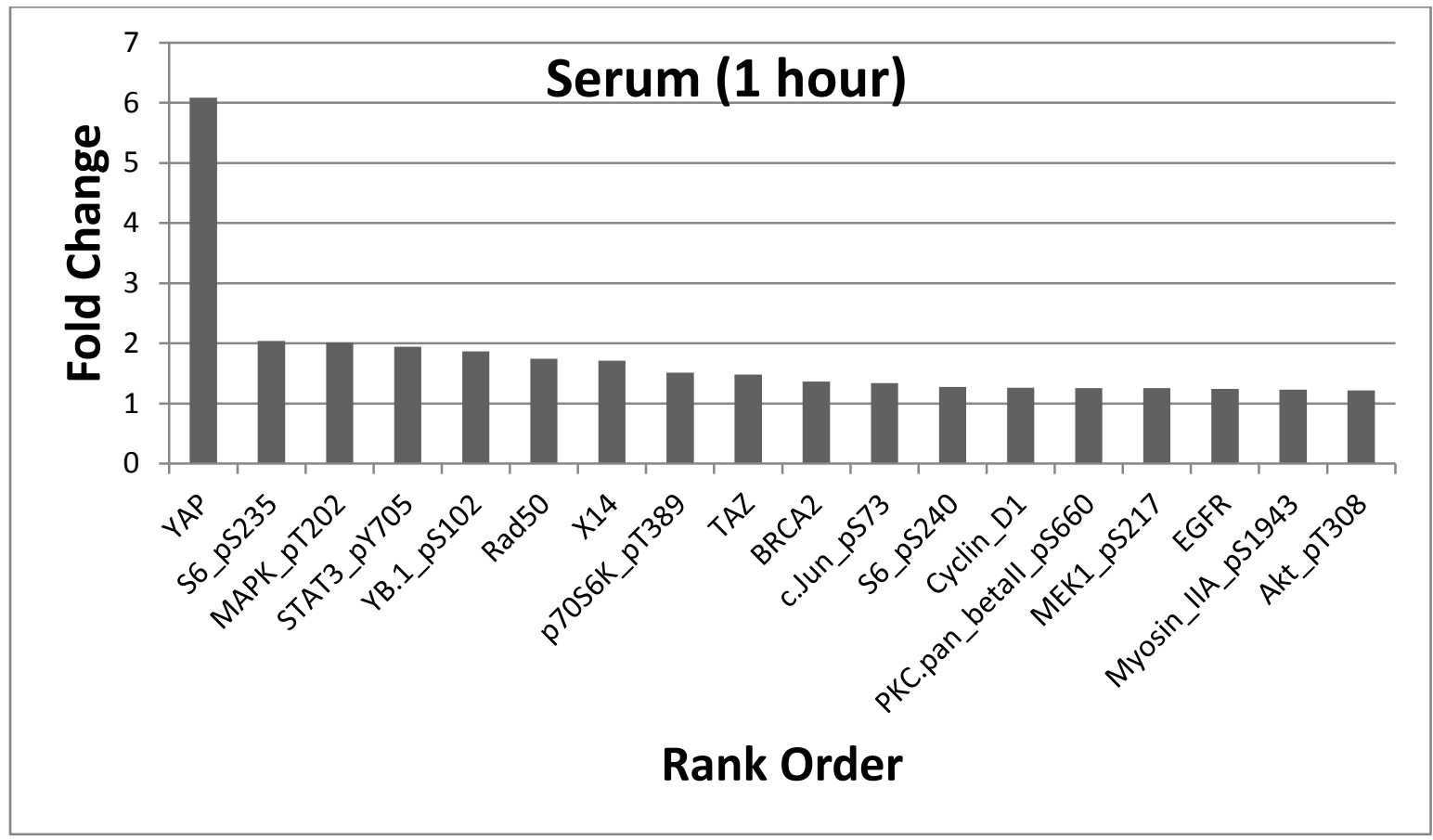

B.

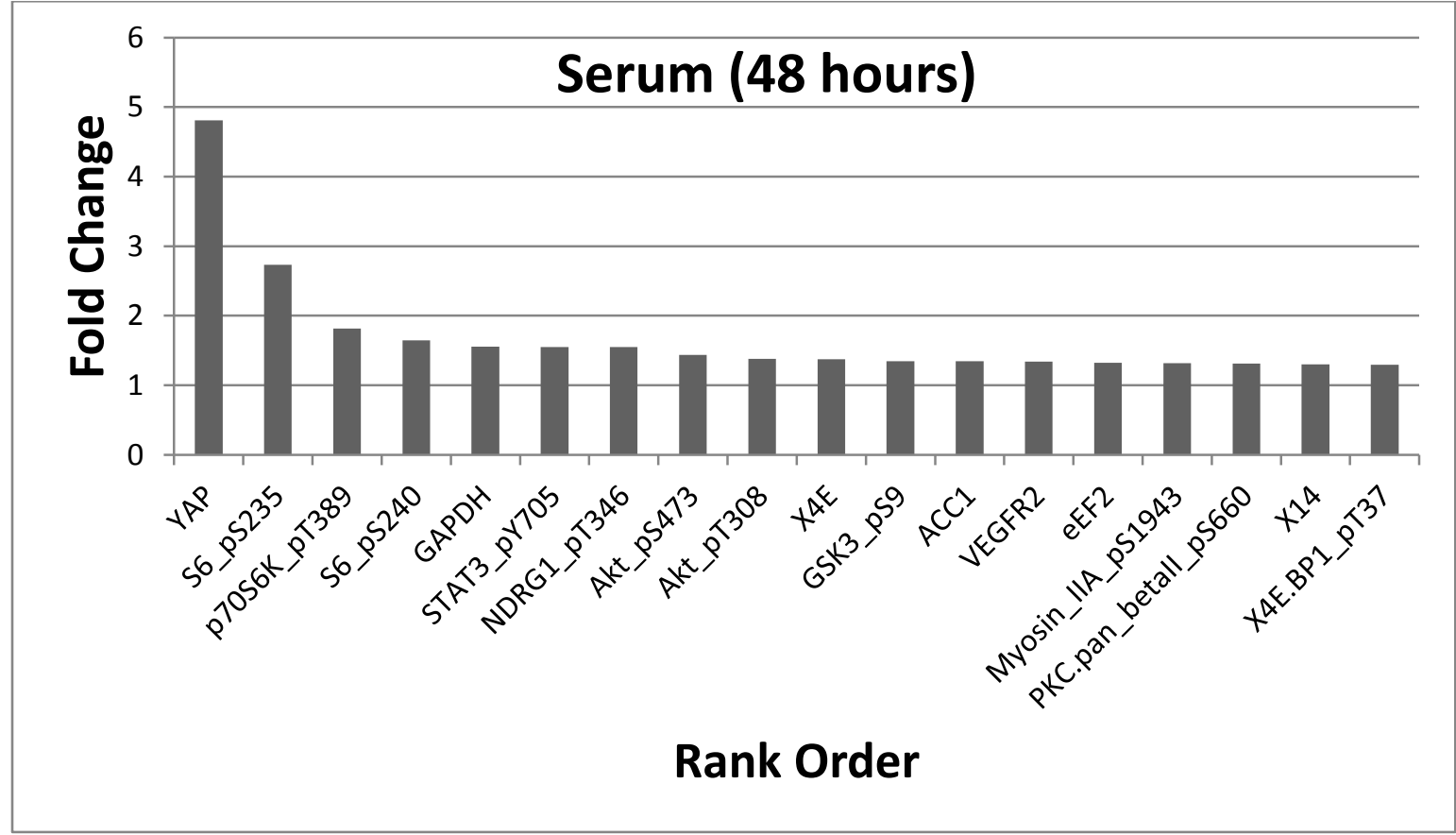

Figure 5.4 Rank ordered list of average fold change in protein concentration compared to control for duplicate samples treated with serum for A) 1 hour and B) 48 hours. 
A.

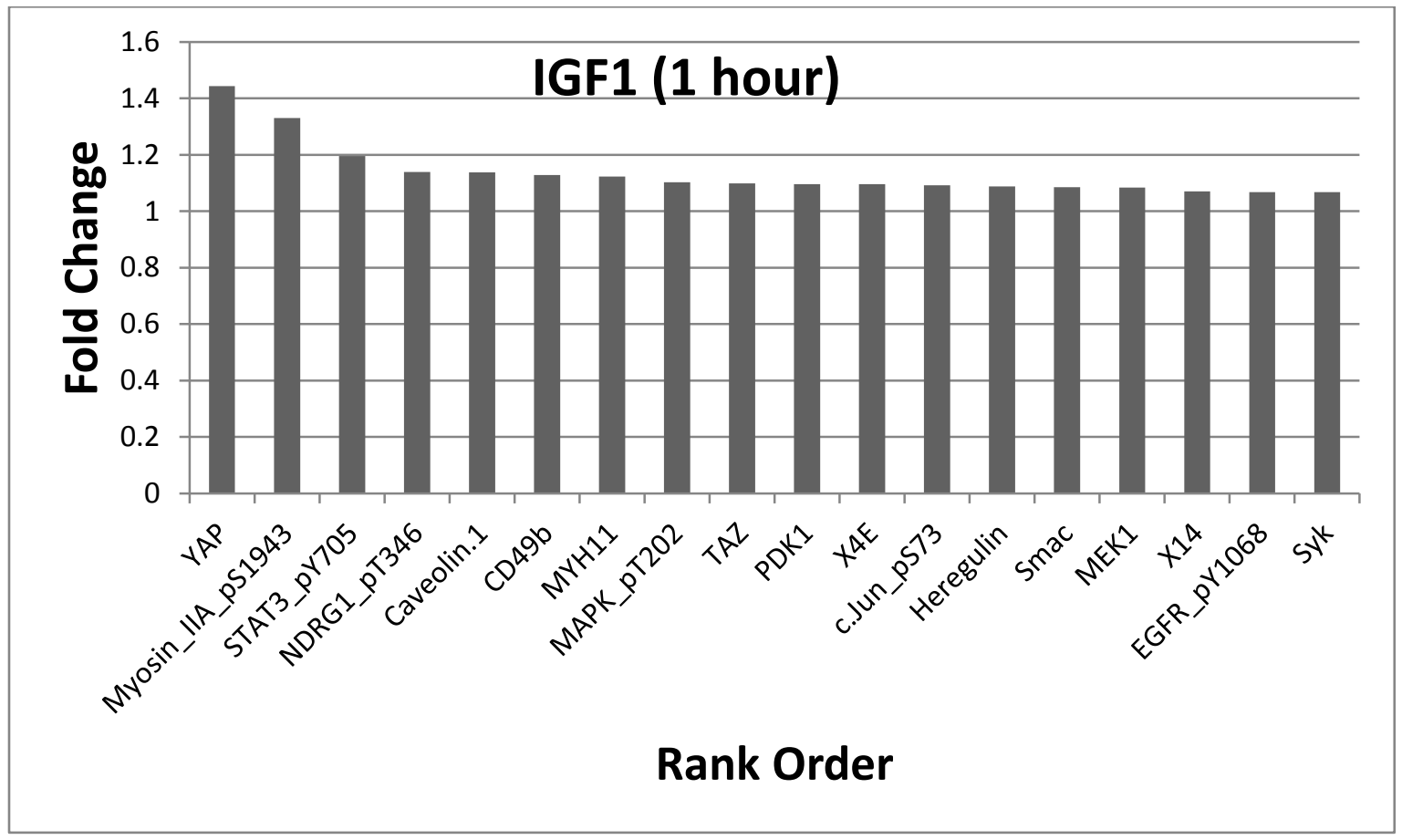

B.

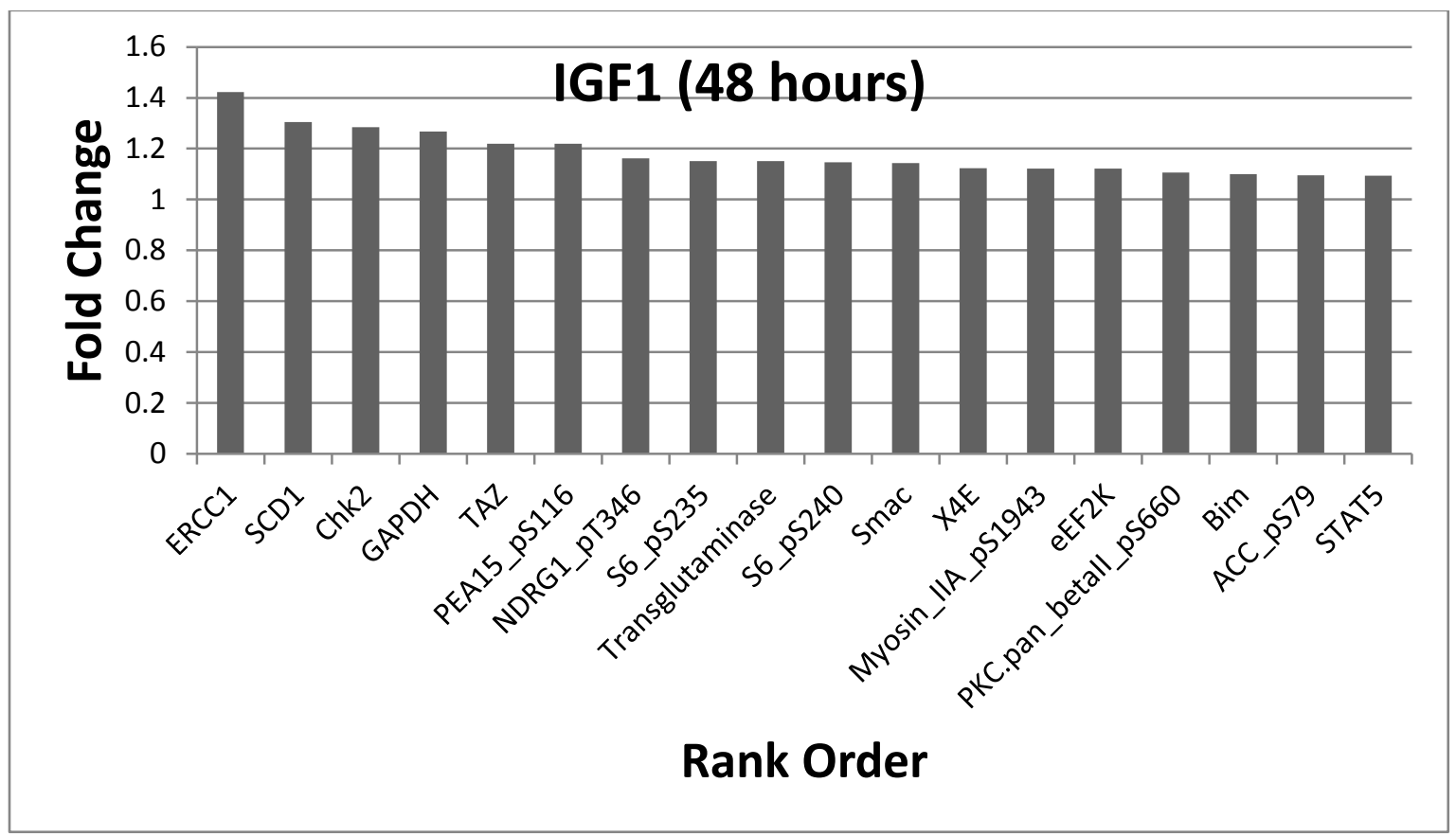

Figure 5.5 Rank ordered list of average fold change in protein concentration compared to control for duplicate samples treated with 10 nM IGF1 for A) 1 hour and B) 48 hours. 
To increase understanding of the effects of the ligands on the PI3K and Ras signaling pathways we produced heat maps displaying the effect sizes of the hypertrophic agonists on the phosphorylation state of species in these pathways. For PI3K signaling, phosphorylated AKT, GSK3, and mTor are relevant, and for Ras signaling, phosphorylated MAPK, MEK1, p38, JNK, cJun, and cRaf are relevant. We performed hierarchical clustering of the data of average fold change in protein concentration compared to control. Each output (column) was normalized before clustering to give a mean of 0 and a standard deviation of 1 . This allowed us to compare outputs that had different effect sizes.

At 1 hour, we see high AKT signaling with Nrg1 relative to the other ligands and high phosphorylated cRaf with ET1 (Figure 5.6A). Phosphorylated MAP Kinases are elevated with Nrg1, ET1, and Serum. At 48 hours, AKT is increasingly elevated with Nrg1 (Figure 5.6B). Phosphorylated MAP Kinases are elevated with Nrg1 and Serum, but not as high as at the 1 hour time point. Signaling levels were relatively lower with Ang II and IGF1. Ang II did not show substantial AKT signaling at either time point and had moderately upregulated MAP Kinase signaling at both time points. Although we would expect potent AKT signaling with IGF1[152], we were not able to measure many large changes in signaling in the neonatal myocytes.

In the combined time point heat map we see high correlation between phosphorylated AKT and GSK3 and between many of the MAP Kinase species: MEK1, MAPK, and p38 (Figure 5.7). This is consistent with what we would expect since these species are in the same signaling pathway. mTor, which is in the PI3K signaling cascade, however, is more similarly correlated to MAP Kinase signaling than AKT signaling. Moreover, cRaf, which is in the Ras signaling pathway, exhibits unique signaling compared to the other MAP Kinase species.

While future work manipulating the levels of Ras and PI3K signaling in the myocytes is needed to learn more about the role of these species in specific maladaptive and adaptive hypertrophy features, it 


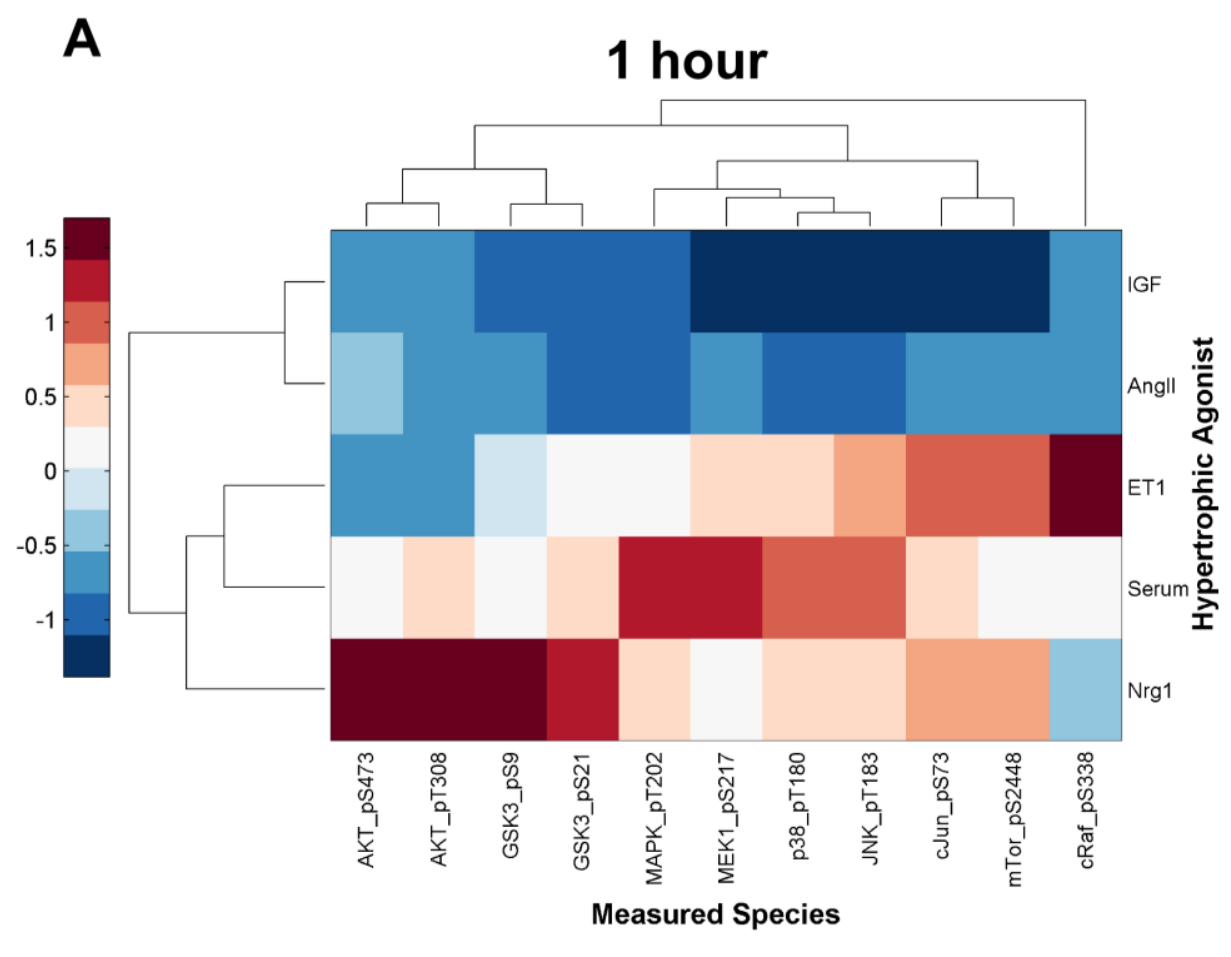

B 48 hours

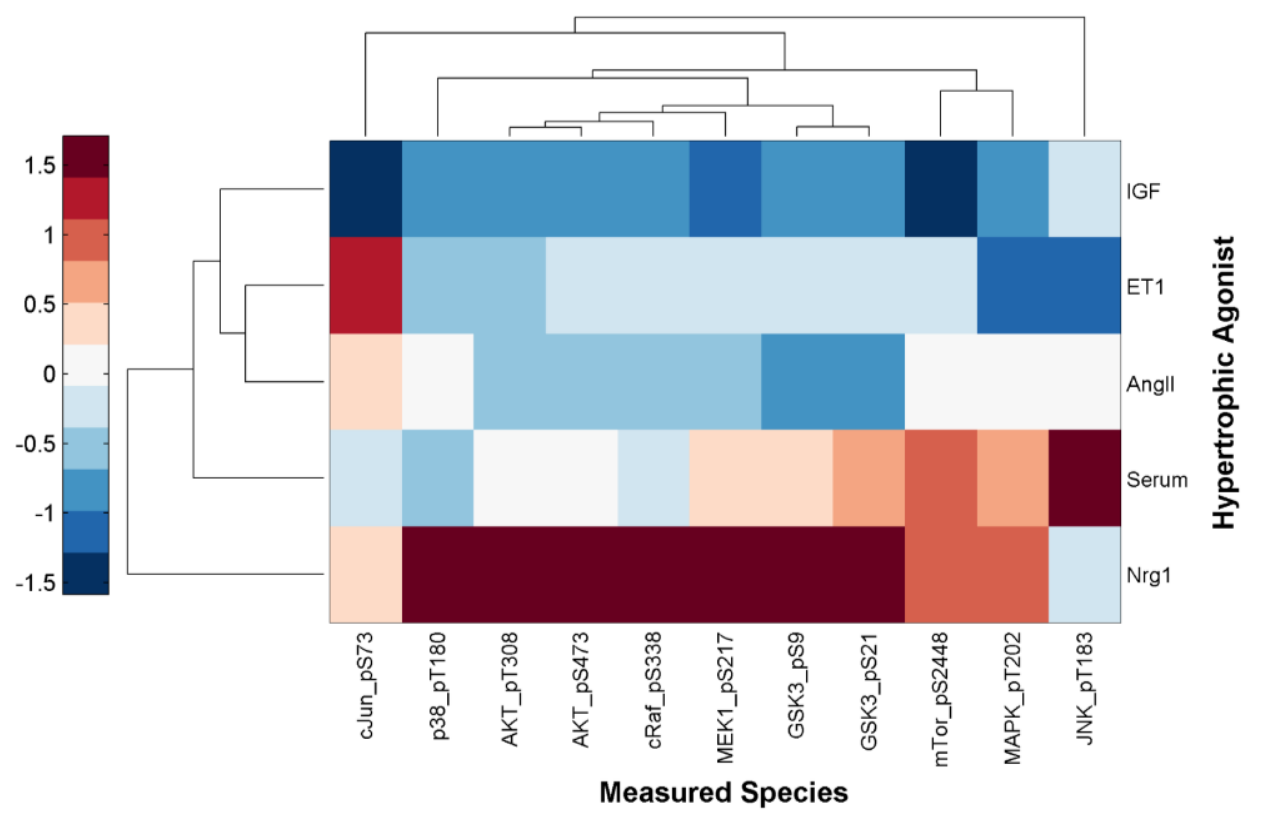

Figure 5.6 Hierarchical clustering of the RPPA data at A) 1 hour and B) 48 hours of average fold change in measured protein concentration of species in the Ras and PI3K signaling pathways compared to control. 


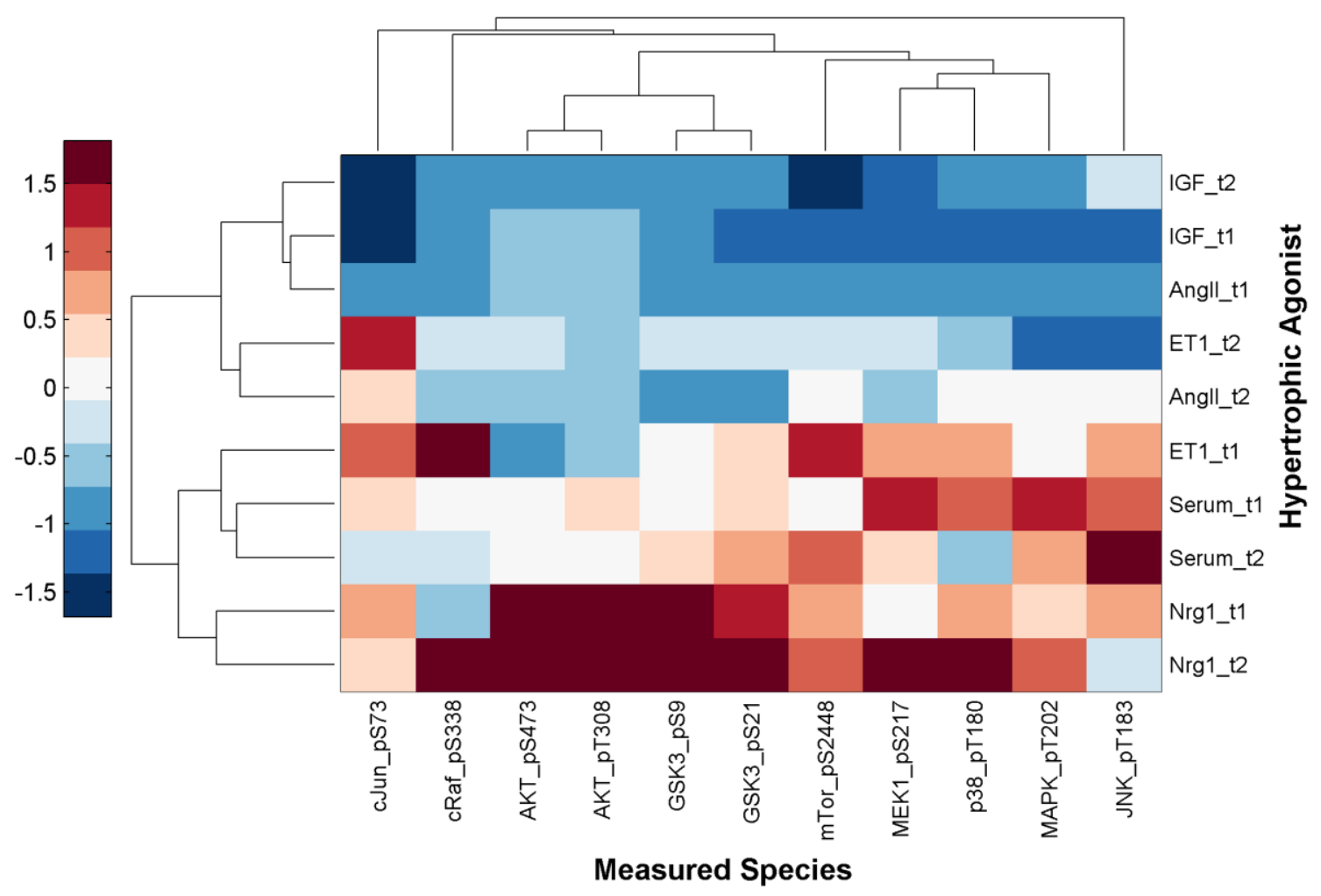

Figure 5.7 Hierarchical clustering of the combined RPPA data at both 1 hour ( 11 ) and 48 hours (t2) of average fold change in measured protein concentration of species in the Ras and PI3K signaling pathways compared to control. 
is clear that these species, which have unique effects on myocyte phenotypes (Chapter 4), also have differences in relative amounts of Ras and PI3K signaling. Nrg1 has the highest levels of AKT signaling, Ang II has absent AKT signaling, and ET1 and Serum have elevated AKT and Ras signaling, but at different time points, where increased AKT signaling occurs later. 
Chapter 6

Dissertation conclusions and future directions 


\subsection{Conclusions}

The overall goal of this dissertation was to integrate experimental and computational approaches to determine how the components and network topology of hypertrophy signaling lead to differential regulation of myocyte shape and gene expression. In meeting this objective, we have

i.) Developed an automated microscopy and image processing platform for cardiac myocyte hypertrophy.

ii.) Discovered a delay in reversal of phenylephrine-induced hypertrophy due to intracellular $\alpha$ adrenergic receptors.

iii.) Built a large-scale computational model of the cardiac myocyte hypertrophy signaling network and used the model to identify the most influential species in the network.

iv.) Predicted and experimentally validated Ras as a highly influential network hub with distributed regulation of myocyte hypertrophy downstream of Ras.

v.) Identified distinct hypertrophy phenotypic signatures for 15 hypertrophic agonists and outputs with similar patterns of regulation.

vi.) Discovered CITED4 regulation of myocyte elongation and CTGF regulation of Bax mRNA expression.

In Chapter 2 (Aim 1), we described the development of a high-throughput screening procedure to study hypertrophy signaling network in live cardiac myocytes. Using this approach, the shape of thousands of individual cells can be measured and tracked over several days. We validated this imaging platform by studying the dynamics of reversal of PE-induced hypertrophy at multiple concentrations. In doing this, we discovered a concentration dependent delay in reversal of hypertrophy that can be mechanistically explained by internalized PE acting on intracellular $\alpha$-adrenergic receptors. This memory mechanism, while potentially providing cardioprotection for future insults, could also accelerate the 
development of hypertrophy and heart failure. It also suggests that transient release of circulating catecholamines could lead to sustained hypertrophy signaling. Moreover, $\alpha A R$ agonist internalization would complicate therapeutic approaches aimed at blocking this pathway. Therefore development of pharmacologic agents that can be readily internalized by the cell or prevent uptake of hypertrophic agonists may be important for successful treatment of cardiac hypertrophy. Functional intracellular receptors have also been identified for angiotensin II [63], endothelin-A and -B [64], and $\beta$-adrenergic receptors [65] in cardiac myocytes.

In Chapter 3 (Aim 2), we described the development of a 106-species computational model of the cardiac myocyte hypertrophy signaling network. Using the model we identified Ras as the most influential species in the network, followed by JAK, G $\beta$, gp130LIFR, and Gaq. Notably, correlations between local connectivity and influence were relatively low, indicating the need for sensitivity analysis of the network to identify these highly influential species. Motif analysis revealed an enrichment of bifan and bi-parallel motifs, network motifs that could accelerate the development of hypertrophy and impede its reversal. Moreover, the modules we identified in the network through clustering of the sensitivity analysis data were also found to be highly interconnected, further demonstrating the high level of cross-talk in the network. While conceptual models of hypertrophy signaling often depict independent linear pathways, we showed that cardiac hypertrophy signaling is a highly integrated network.

In Chapter 4 (Aim 3), we described the results of a phenotypic screen of 15 predominant hypertrophic agonists where we measured fold changes in 5 shape features and transcript levels of 12 genes. In validating our computational model of hypertrophy we observed that commonly measured markers of hypertrophy such as cell size and fetal gene expression are not markedly differentially regulated between receptor pathways in the signaling network. When we expanded the number of measurements to include transcripts related to elongation, fibrosis, cell death, proliferation, and 
inflammation, we were able to measure distinct hypertrophy phenotypic signatures among the receptor agonists. Five modules were identified within inputs and outputs, with many maladaptive outputs grouping together in one module (Bax, C/EBP $\beta$, Serca2a, TNF $\alpha$, and CTGF). Shared regulation of maladaptive hypertrophy features could provide insight into the observation of distinct in vivo presentations of pathological and physiological hypertrophy. Additionally, protective outputs VEGF and $\mathrm{Bcl} 2$ were in a module with measures of cell size and hypertrophy (fetal gene program). The screen also revealed correlation between CITED4 transcript abundance and elongation. Follow-up experiments revealed that CTGF regulates Bax expression and that CITED4 negatively regulated myocyte elongation and induces physiological hypertrophy. Therapies targeting pro-fibrotic CTGF may therefore have added benefits by reducing cell death. Moreover, CITED4, which has been shown in a previous study to induce myocyte proliferation, may be a promising therapeutic target for heart failure by protecting myocytes from elongation, which is associated with poor clinical outcomes.

These aims helped reveal mechanisms that control how the hypertrophy signaling network manages a variety of hypertrophy phenotypic outputs. We identified important network hubs and shared regulation of maladaptive and adaptive hypertrophy features. While this systems approach revealed insights into network organization, it also allowed us to prioritize experiments to reveal new mechanistic insights into hypertrophy, such as the discovery of CITED4 regulation of myocyte elongation. The quantitative network understanding gained in this work will be helpful in planning therapeutic interventions for heart failure that enhance adaptive responses and suppress maladaptive responses.

\subsection{Future Directions}

Fortunately, many opportunities remain for future work studying network organization in cardiac myocyte hypertrophy. Here we developed a high-throughput imaging platform and large-scale 
computational model of hypertrophy. These tools can be readily applied to study other important features in heart failure such as fibrosis, cell death, and proliferation. We saw in the single ligand screen that many predominant hypertrophic agonists also affect these phenotypic outputs, so there will likely be a lot of overlap between computational models of hypertrophy, fibrosis, cell death, and proliferation. It will be interesting in future work to discover which pathways are shared versus independent in controlling these different features of cardiac remodeling. Development of high-throughput methods for measuring these features in vitro will be critical in producing the data needed to construct these other models. Since cell boundary information is less critical in measuring these other features, plate reader assays of markers for cell death, fibrosis, and proliferation could be useful in quickly screening compounds.

In our phenotypic screen follow-up study we discovered that CITED4 negatively regulated myocyte elongation and induced physiological hypertrophy. To evaluate CITED4's potential as a therapeutic target, it will be important in future studies to determine CITED4's role in regulation of adaptive phases of eccentric hypertrophy versus maladaptive phases that lead to heart failure. This can be studied by increasing cardiac expression of CITED4 in mice using an established method for cardiac myocyte-specific gene delivery: AAV-9 mediated expression of CITED4 from the cardiac troponin T promoter[47]. Mice could then be given a physiological or pathological volume overload stimulus such as aerobic exercise or myocardial infarction, respectively. Comparing chamber dimensions to respective controls will elucidate the effects of CITED4 on physiological and pathological myocyte elongation. Moreover, it will be essential to confirm the effectiveness of CITED4 expression as a therapeutic target in vivo. This could be done by giving mice myocardial infarctions and comparing the hemodynamic and histological parameters of mice with cardiac overexpression of CITED4 versus control. 
We used our high-throughput imaging platform to study shape changes in neonatal rat ventricular myocytes induced by 15 receptor agonists. These techniques can be readily extended to be used in high-throughput RNAi screens or with human stem-cell derived cardiac myocytes. An RNAi screen would be useful in discovering genes regulating different aspects of myocyte shape such as elongation. It will be useful to confirm screen hits in human stem-cell derived cardiac myocytes to see if findings in neonatal rat myocytes translate to human cardiac myocytes. Confirmation of key results in human cells could be a useful preliminary study before pursuing a target in vivo.

Future work is also needed to fit the parameters of the large scale computational model of hypertrophy. Currently the model uses a normalized Hill equation modeling framework with default parameters, which allowed us to build a comprehensive model of the signaling network. We validated the model by comparing qualitative input - output relationships for transcription factor activities and phenotypic outputs such as cell size and fetal gene expression and saw $96 \%$ agreement. In our receptor agonist screen, we observed large differences in the effect on cell size among the ligands. The $\alpha$ and $\beta$ adrenergic receptor agonists (ET1, NE, PE, and ISO) induced much greater changes in cell size than the other agonists. The current version of our model, however, predicts much more similar cell size responses among the various ligands (Figure 6.1). This is likely due to the high level of cross-talk in the network, such that agonists activate similar species (Figure 6.2). Therefore, future work is needed to fit reaction weights so that our model is able to make more quantitative predictions about the relative contribution of receptor agonists to hypertrophy. Given the high amount of cross-talk, key reactions to fit will likely be at the receptor level. Cardiac myocyte cell size and shape data produced from screens of drug libraries inhibiting various species in the network will also be helpful in expanding the validation of our model and tuning the weights of model reactions. 

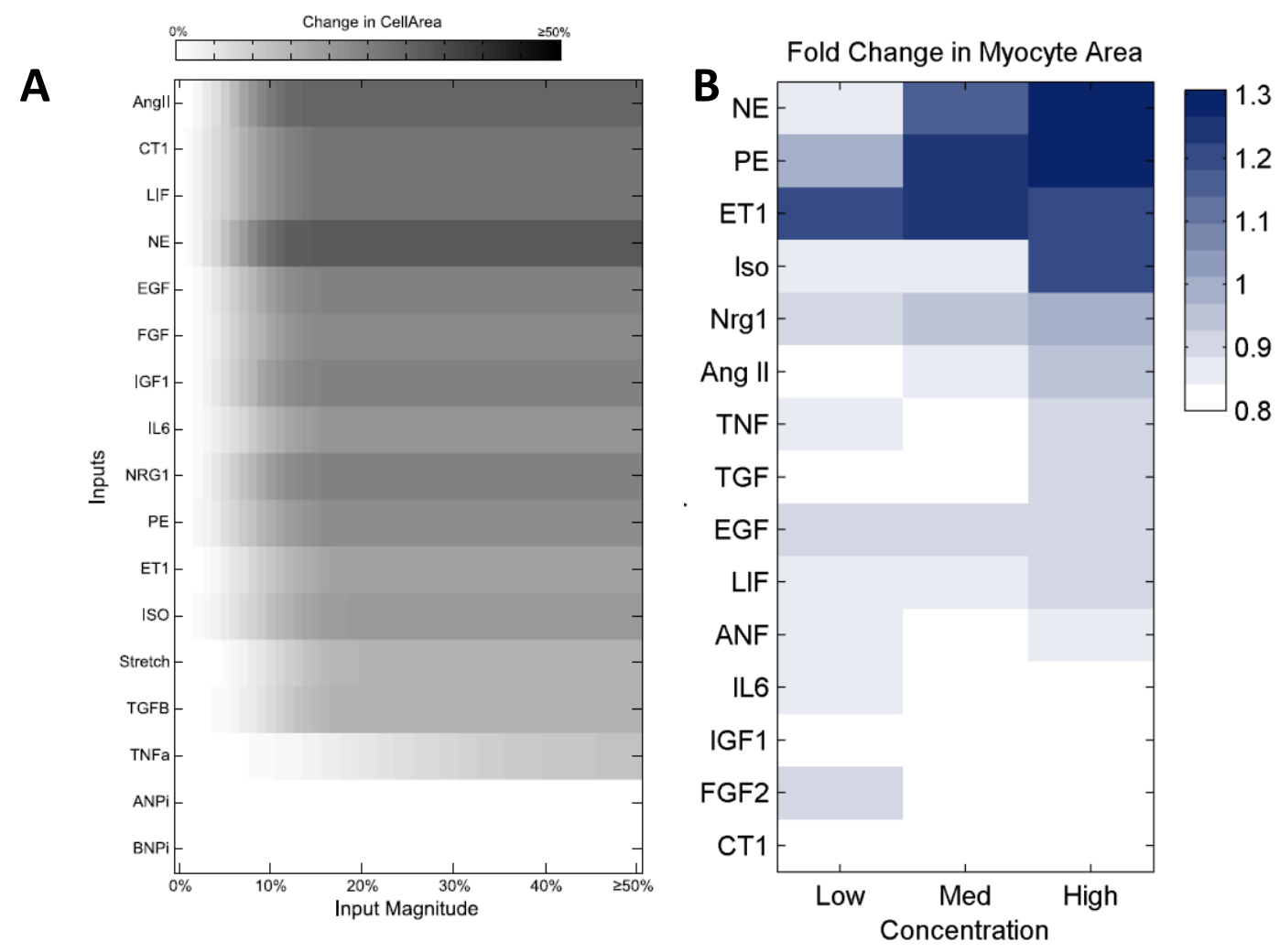

Figure 6.1: A) Predicted magnitude of change in cell area in response to increasing levels of input activation by the hypertrophy signaling network computational model. Y-axis is organized by EC50. B) Ligand screen data of magnitude of change in cell area in response to increasing levels of receptor agonist. PE, ET1, and Iso had much larger effects on cell area than the current model is able to predict. 


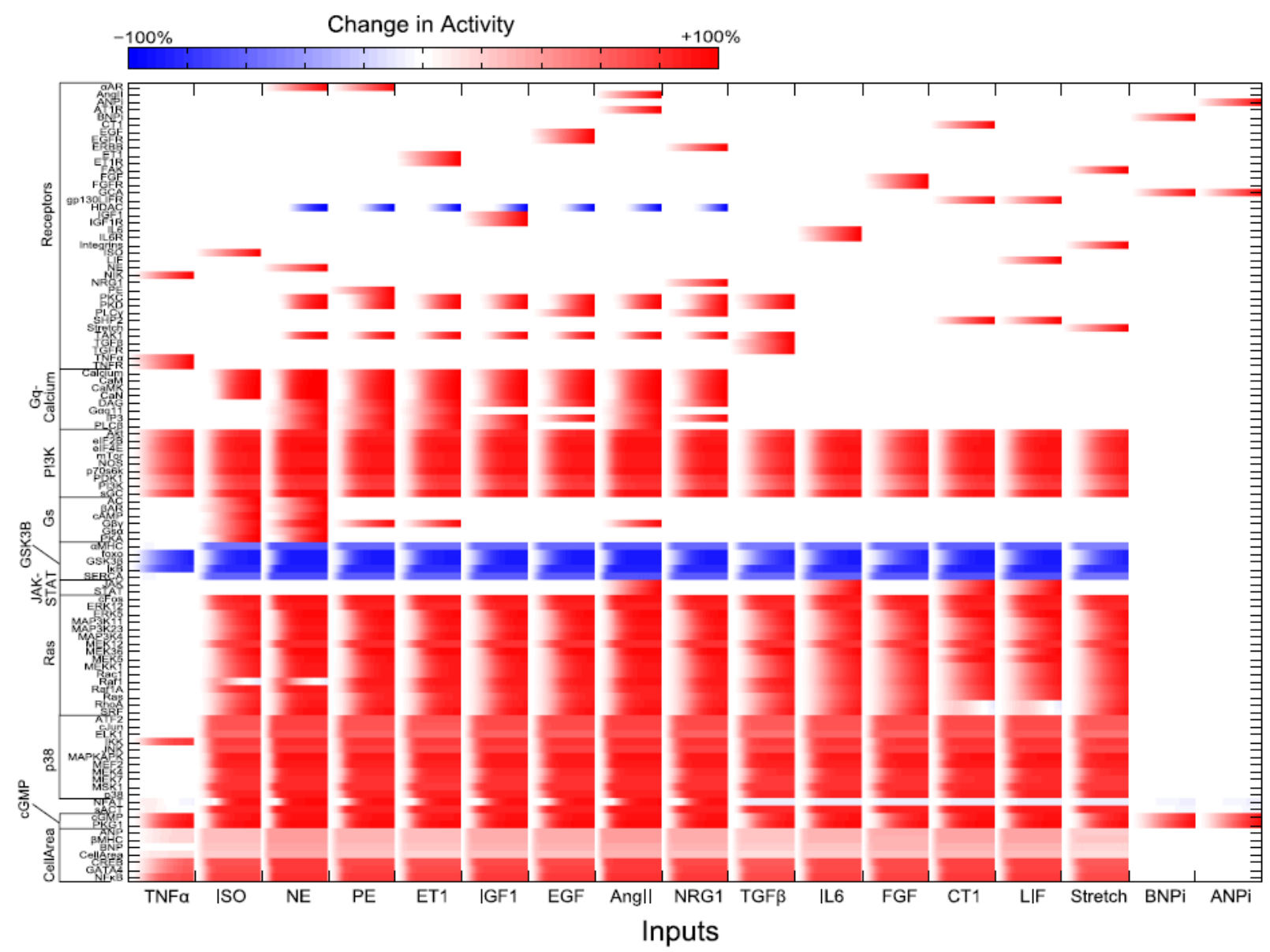

Figure 6.2 Predicted hypertrophy signaling network dose response at input concentrations ranging from 0 to $100 \%$. Network species ( $y$-axis) are organized by module. Most of the modules are affected by every receptor agonist due to high network cross-talk. 
Finally, continuing work is needed to learn about the role of key hypertrophy network hubs in differential regulation of hypertrophy features. In the ligand screen we were able to measure distinct hypertrophy phenotypic signatures and correlations among various outputs. Further work is needed to discover the intermediate species that control the expression of adaptive and maladaptive genes. Preliminary data from our computational model of hypertrophy point to two initial signaling hubs to test: Ras and PI3K. The model analysis revealed a new conceptual model where receptors act in a crosstalk dense network, with many signals funneling through these two network hubs.

The role of Ras and PI3K in hypertrophy signaling can be studied by stimulating neonatal rat ventricular myocytes with a diverse subset of hypertrophic agonists, such as ET1, Nrg1, and Angll, and pharmacologically inhibiting Ras or PI3K. Unfortunately, specific Ras inhibitors are unavailable, so downstream map kinases like MEK1 and p38 will need to be targeted instead. 500 nM PI-103 inhibits PI3K, 100nM PD325901 inhibits MEK, and $10 \mu \mathrm{M}$ SB203580 inhibits p38. Shape and gene expression can be measured as we did for the single ligand screen in Chapter 4. We hypothesize that these inhibitors in the PI3K and Ras pathways will have different effects on maladaptive and adaptive hypertrophy features. We hypothesize that high Ras (high MEK and/or p38) signaling relative to PI3K will result in more pathological features such as increased elongation, fibrosis, cell death, and that high PI3K signaling relative to Ras will result in more protective hypertrophic features such as proliferation, growth without elongation, protection from cell death, and myocyte proliferation. 


\section{Appendix A}

\section{Detailed methods for high-throughput cardiac myocyte image acquisition and analysis.}




\section{A.1 Cell Culture}

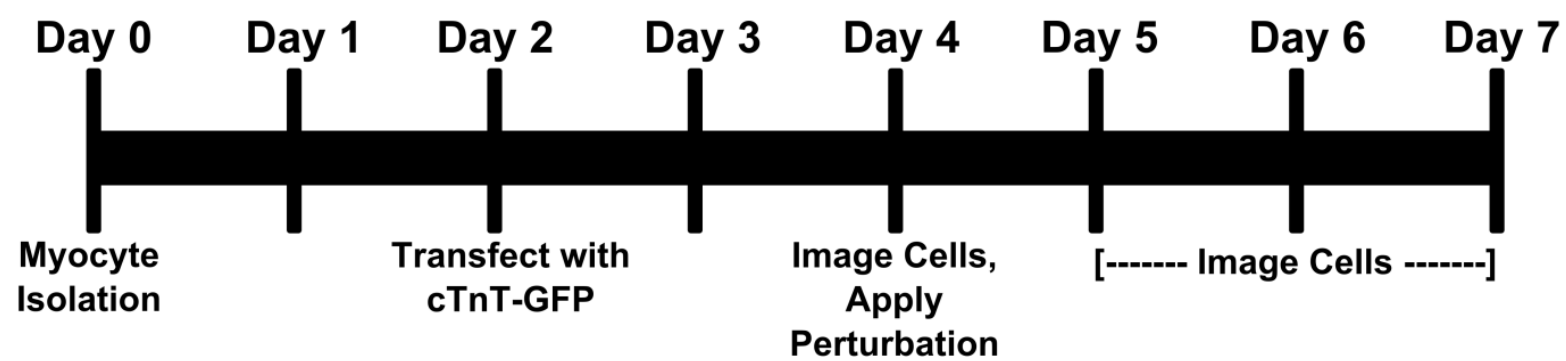

Figure A.1: Timeline for cell culture and imaging for automated imaging of changes in cardiac myocyte shape.

1. Isolate rat cardiac myocytes from 1-2 day old Sprague Dawley rats using the Cellutron Neonatal Cardiomyocyte Isolation kit.

2. Plate myocytes on 96 -well plate coated with SureCoat, 100,000 myocytes per well.

3. Culture myocytes in cell culture media at $37^{\circ} \mathrm{C}$ and $5 \% \mathrm{CO}_{2}$.

4. 2 days after isolation, transfect cardiac myocytes with $0.4 \mu \mathrm{g}$ GFP under a troponin T promoter per well using Lipofectamine 2000 according to the manufacturer's instructions (see Note 1). Wait 48 hours after transfection for adequate GFP expression for imaging (see Note 2).

5. After collecting initial images, rinse the myocytes and then culture myocytes in cell culture media without serum with the desired perturbation (see Note 3).

6. Record follow-up images at the desired time points. Myocytes can be reliably imaged for about a week.

7. A complete cell culture timeline for transfection and imaging is shown in Figure A.1. 


\section{A.2 Image Acquisition}

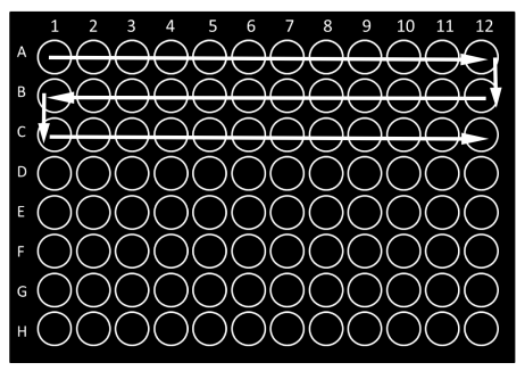

1. Move to next well

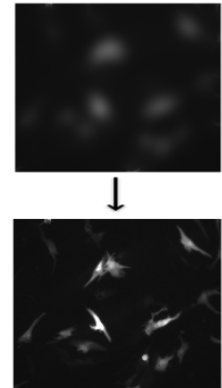

2. Auto Focus

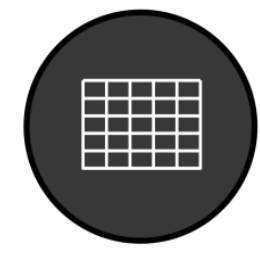

3. Set imaging coordinates

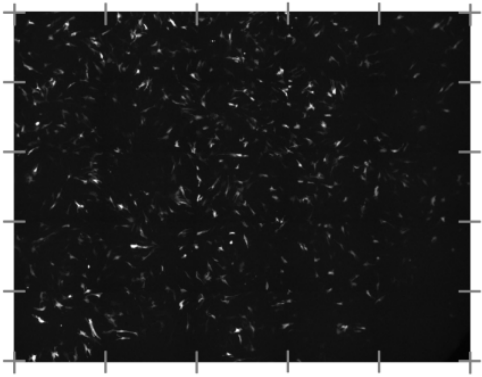

4. Acquire $5 \times 5$ mosaic image

Figure A.2: Scripts to control the microscope automated stage allow for quick and reproducible imaging of the same set of myocytes over time. Using pre-saved coordinates for the centroid of each well, the automated stage is programmed to move to each well of interest in a 96-well plate. After (1) moving to the next well, the algorithm will (2) autofocus, (3) set imaging coordinates depending on the size of the mosaic image and (4) acquire the mosaic image. A representative $5 \times 5$ mosaic image is shown.

Software algorithms were developed to automatically focus and collect 25 images in each well of interest in a 96-well plate (Figure A.2). These 25 images are put together into a composite $5 \times 5$ mosaic image for each well. By programming the automated microscope stage, the stage moves to the center of each well automatically.

Many microscopy image acquisition software packages allow users to develop scripts to automate experiments. The basic workflow for scanning 96-well plates to measure changes in cell shape is as follows:

1. Save coordinates of centroids of each well of interest in your 96-well plate.

a. Metamorph has a "scan multi-well plate" command which allows user to specify the dimension of the plate and then select which wells the user wants to image. Save the stage position coordinates of the first well to standardize the path of the scan. The user would then move the stage to this saved location at the beginning of each experiment.

b. If your software package does not have a similar built-in function, you can create an array of stage position coordinates, saving the centroid locations of each well.

$$
\sim 150 \sim
$$


2. Using these well coordinates, develop a script that moves the stage to each location you want to image. Moving the stage in a serpentine pattern allows for the least amount of total stage movement.

a. Again, this can be done in Metamorph using the "scan multi-well plate" function with the plate dimensions.

3. Set an image acquisition script to execute in each well. In Metamorph, these script files are called journals. The script should have the following commands:

1. Coarse autofocus - Move objective to five different heights, $10 \mu \mathrm{m}$ apart (+/- $20 \mu \mathrm{m}$ from the current objective height) and automatically select the height producing the image with the sharpest image resolution. In order for this algorithm to be successful, the focus must be approximated by the user at the beginning of the experiment.

a. In Metamorph this operation can be accomplished using the "Adjust Focus" command in the Journal Editor.

2. Fine autofocus - Move objective to five different heights, $2 \mu \mathrm{m}$ apart (+/- $4 \mu \mathrm{m}$ from the current objective height) and automatically select the height producing the image with the sharpest image resolution.

a. In Metamorph this operation can be accomplished using the "Adjust Focus" command in the Journal Editor with different parameter values.

3. Set parameters for the imaging bounds within the well. If you would like to collect more than 1 image per well, the user needs to set up the boundary coordinates for imaging within the well.

a. In Metamorph this can be done using the "Assign Variable" command. For a 5x5 image area:

i. Set "ScanSlide.ScanUpperLeft.X" to "StagePosition.StageX"-1077 


\section{ii. Set "ScanSlide.ScanLowerRight.X" to "StagePosition.StageX"+1077 \\ iii. Set "ScanSlide.ScanUpperLeft.Y" to "StagePosition.StageY"+800 \\ iv. Set "ScanSlide.ScanLowerRight.Y" to "StagePosition.Stagex"-800}

These numbers can be modified for smaller or larger imaging areas. Larger imaging areas generate more cell data per condition, but require more computing resources to analyze due to the larger file sizes.

4. Collect images using the set parameters.

a. In Metamorph this can be done using the "Scan Slide" command with the above variables set.

b. Before first using Scan Slide, this command needs to be calibrated. Also, image acquisition settings are set in this command such as exposure time and binning. An exposure time of 120 ms works well here. These settings can be saved and loaded before beginning image acquisition.

5. Save collected mosaic image.

a. In Metamorph this can be done using the "Save Using Sequential File Name" command.

Cell size, shape, and fluorescence intensity data from these mosaic images can then be extracted using automated image analysis pipelines. Since myocyte migration is minimal and the coordinates of the 96-well plate are fixed, we can use these scripts to image the same set of myocytes over time. Therefore, we can measure fold changes in shape of thousands of individual myocytes after perturbations. Myocytes can be reliably imaged over the time period of about a week. 


\section{A.3 Image Analysis}
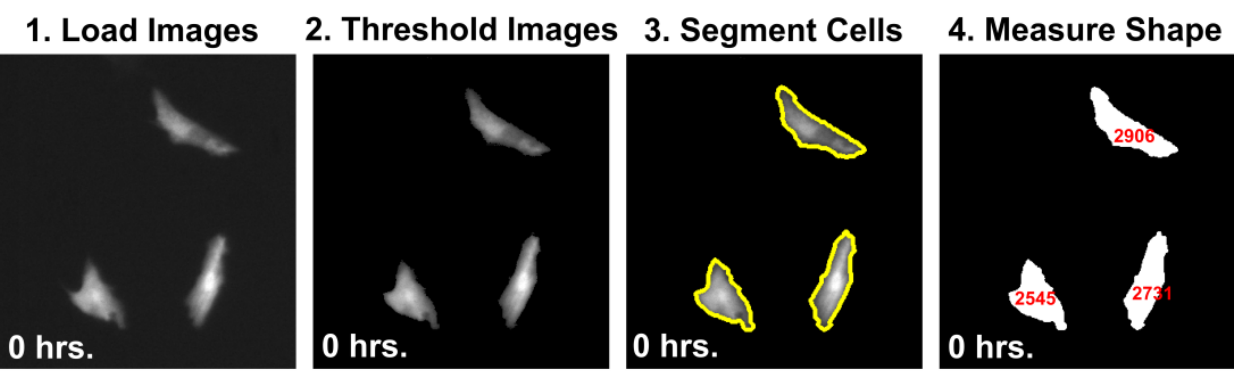

5. Track Myocytes
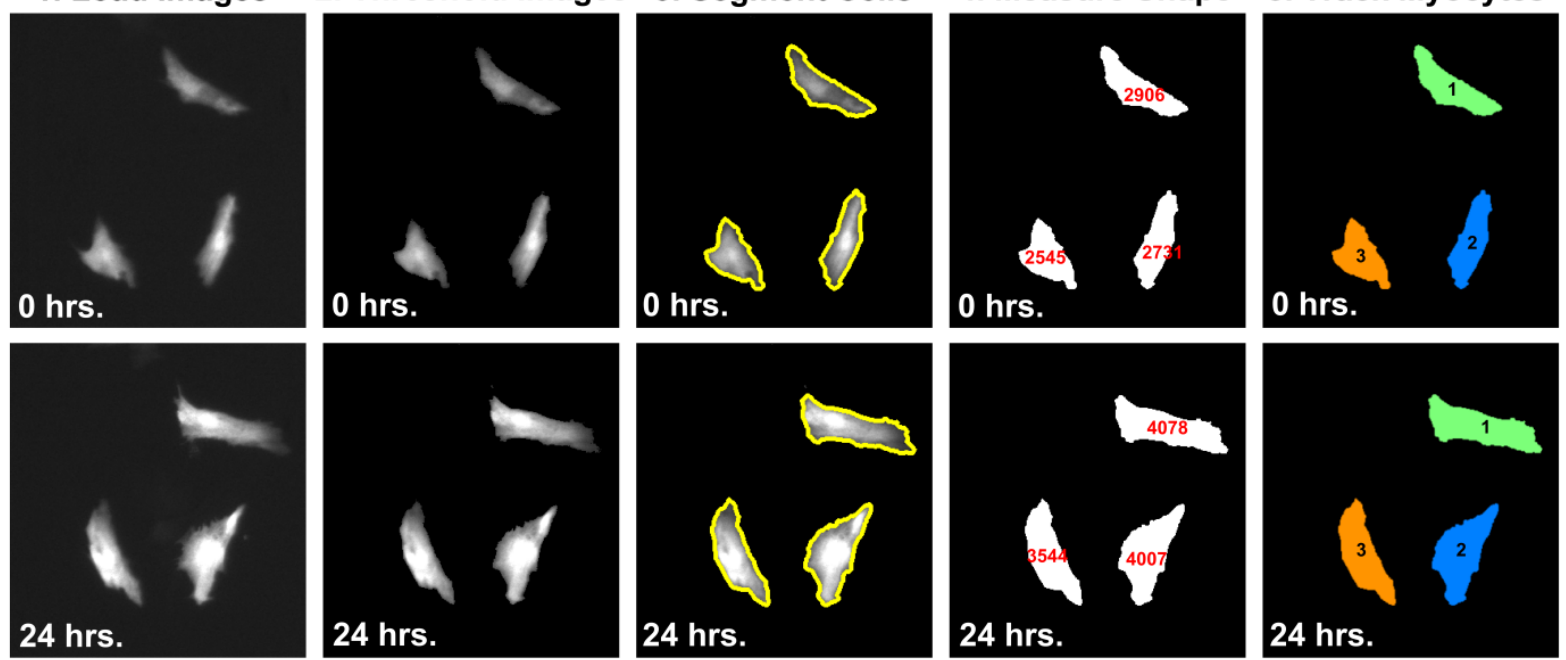

Figure A.3: Image analysis pipelines enable automated quantification of fold changes in shape of cardiac myocytes at multiple time points. The algorithm (1) loads images from each time point of a given well, (2) thresholds the image to reduce background noise, (3) segments myocyte boundaries, (4) calculates shape features such as cell area from the segmentation, and (4) tracks myocytes over time. Myocytes are tracked using the cell with the closest distance to the centroid at the previous time point.

Image analysis scripts can be used to load images, correct for noise, segment myocytes, measure shape and fluorescence intensity, and track myocytes over time (Figure A.3). For labs with more computational experience, the following image analysis procedures can be developed in MATLAB. However, for labs with limited programming experience, the open source program CellProfiler can be used to develop automated image analysis pipelines.

The basic steps of the image analysis pipeline with instructions for implementing this pipeline using CellProfiler are described below. In CellProfiler, image analysis pipelines are built by piecing together built-in image analysis modules. The settings in each module can then be fine-tuned to accommodate the needs of a given image set. A CellProfiler pipeline file and example images for this protocol are freely available at http://bme.virginia.edu/saucerman under Downloads.

1. Load images - Load all images of a given well at each time point measured. 
a. This can be done using the "Load Images" command in CellProfiler and specifying text labels that these images have in common (such as well number) (see Note 4).

2. Background correction - A pixel intensity threshold for image noise is calculated using the Otsu method [48]. This threshold value is then subtracted from the image to remove background noise.

a. Use the "Apply Threshold" command in CellProfiler, set a noise threshold for the image using the "Otsu Adaptive" thresholding method for two classes. Minimize the weighted variance and use a correction factor of 1 (no correction).

3. Segment Myocytes - Calculate a pixel intensity that distinguishes cells from background using the Otsu method. To segment any adjacent cells, first calculate the number of myocytes in the image using the number of local maxima in a smoothed image and then use a watershed algorithm [49] to calculate the dividing lines. Since Lipofectamine 2000 transfection efficiency in neonatal ventricular cardiac myocytes is $10-15 \%$, expressing myocytes are rarely adjacent to each other. Discard myocytes touching the edge of the image from analysis.

a. Use the "Identify Primary Objects" command in CellProfiler with the following specifications:

i. Specify the typical diameter range of the myocytes to eliminate dead cells or noise from the analysis. Discard myocytes outside of this range.

ii. Use Otsu Adaptive thresholding method, two classes, minimizing weighted variance, with a correction factor of 0.8 .

iii. Use "Intensity" to distinguish clumps of objects and draw dividing lines. Since myocytes on average are brighter towards the center, GFP fluorescence intensity is useful in determining the number of myocytes in a 
cluster. The number of local maxima in a smoothed image is used to count the number of myocytes. Then a watershed algorithm is used to set the boundary lines between myocytes.

iv. Specify an appropriate maximum number of objects based on your image sizes and transfection efficiency to prevent program crashes on high noise images.

4. Overlay outlines on original image - Create an image overlaying the segmentation result onto the original image. This can be used to check accuracy of myocyte segmentation.

a. Use the "Overlay Outlines" command in CellProfiler.

5. Measure myocyte size and shape - Use the segmentation result to calculate shape features for each myocyte including area, perimeter, form factor, major and minor axis length, and orientation. For studying hypertrophy, we focused on changes in myocyte area.

a. Use the "Measure Object Size Shape" command in CellProfiler.

6. Measure myocyte GFP fluorescence intensity - Measure the integrated fluorescence intensity of each myocyte based on the segmentation. Looking at integrated intensity reveals information about the amount of expression of the plasmid. Certain perturbations may increase the expression depending on the promoter.

a. Use the "Measure Object Intensity" command in CellProfiler.

7. Track myocytes between days - Give each myocyte an identification number and then find each myocyte's position in images at later time points by identifying the closest myocyte to its original position (see Note 5).

a. Use the "Track Objects" command in CellProfiler with "Distance" as the tracking method. This gives each myocyte an identification number and identifies which myocyte in subsequent images is the same cell. The pixel neighborhood the 
algorithm searches around each myocyte on later days is specified. 150 pixels is a good starting value. These identification numbers then can be used to sort the myocyte measurements in post-processing.

8. Save image processing images - Save images from desired points in image processing algorithm such as the image with the segmentation outlines.

a. Use the "Save Images" command in CellProfiler to save the image with cell outlines and the image with cell ID numbers from the tracking algorithm.

\section{A.4 Notes}

1. Myocytes may also be transfected 24 hours after isolation with similar results.

2. While GFP fluorescence intensity increases over the course of the experiment, these increases have minimal effects on area measurements $(<5 \%)[39]$.

3. After initial images are collected, it is important to wash and transfer myocytes to media without serum since serum causes high levels of myocyte growth.

4. Depending on the file type the images are saved as by the image acquisition software, the images may need to be converted to a different file type. This can be done using the batch convert plugin in ImageJ software. CellProfiler can read .png, .tif, .bmp, and .jpg.

5. Myocytes migrate minimally and can be robustly tracked using distance from the previous location. However, if follow-up images are > 100 pixels out of alignment, the tracking algorithm will fail. Therefore, image alignment should be checked and corrected before running the algorithm. If images are dramatically out of alignment, pre-process images in a new CellProfiler pipeline using the "Align" module or align by batch cropping images from each time point in ImageJ. 


\section{Appendix B}

\section{PE ligand trapping model}


Equations for ligand trapping model:

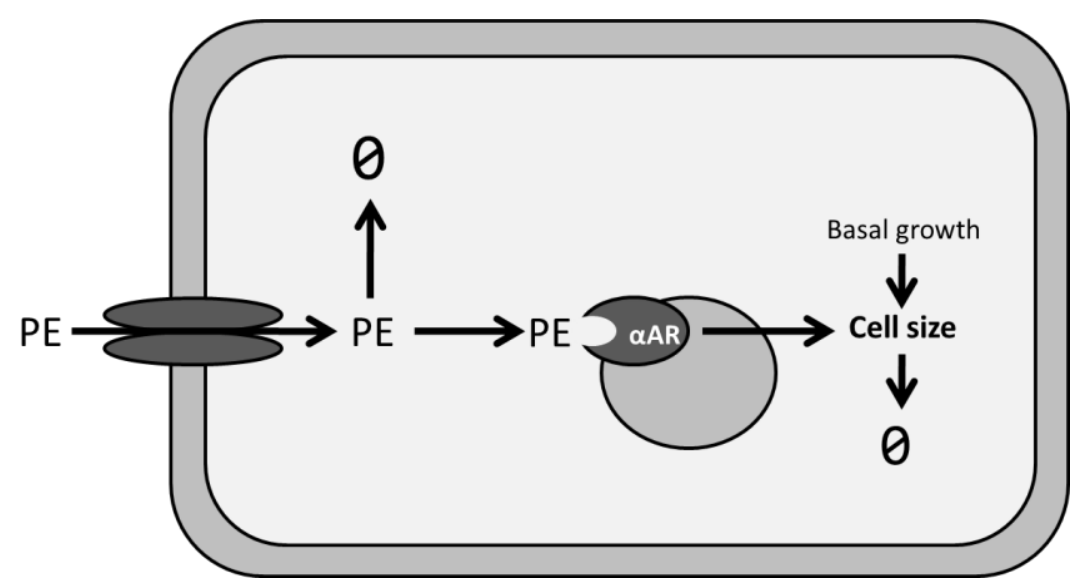

Parameters:

$\mathrm{k} 1=\mathrm{PE}$ internalization rate $\left(\right.$ hours $\left.^{-1}\right)$

$\mathrm{k} 2$ = maximum $\mathrm{PE}$ degradation rate $\left(\right.$ hours $\left.^{-1}\right)$

$\mathrm{k} 3$ = maximum rate of PE-induced cell growth (hours ${ }^{-1}$ )

k4 = Rate of reversal of cell growth (hours ${ }^{-1}$ )

$\mathrm{k} 5$ = basal growth rate $\left(\frac{\mu \mathrm{mol}}{\mathrm{L} * \text { hours }}\right)$

$\mathrm{Kd}=\mathrm{PE}$-induced cell growth $\mathrm{Km}(\mu \mathrm{mol} / \mathrm{L})$

$\mathrm{Kd} 2=\mathrm{PE}$ degradation $\mathrm{Km}(\mu \mathrm{mol} / \mathrm{L})$

Parameter Fitting Results:

The following parameters were fit to the data in Figure 2a:

$\mathrm{k} 1=0.3771$ hours $^{-1}$

$\mathrm{k} 2=221$ hours $^{-1}$

$\mathrm{k} 3=0.0181$ hours $^{-1}$

$\mathrm{k} 4=0.0119$ hours $^{-1}$

$\mathrm{k} 5=.008 \frac{\mu \mathrm{mol}}{\mathrm{L} * \text { hours }}$

$\mathrm{Kd}=1.6682 \mu \mathrm{mol} / \mathrm{L}$

$\mathrm{Kd} 2=746 \mu \mathrm{mol} / \mathrm{L}$

Differential Equations:

$\frac{d P E_{\text {in }}}{d t}=k 1 * P E_{\text {out }}-\frac{k 2 * P E_{\text {in }}}{K d 2+P E_{\text {in }}}$

$\frac{d \text { CellSize }}{d t}=\frac{k 3 * P E_{\text {in }}}{K d+P E_{\text {in }}}-k 4 *$ CellSize $+k 5$

$\mathrm{PE}_{\text {out }}$ was held constant at $0,0.1,1,10,100$, or $1000 \mu \mathrm{mol} / \mathrm{L} \mathrm{PE}$ for 24 hours and then set to $0 \mu \mathrm{mol} / \mathrm{L}$ for all conditions for 48 hours to simulate extracellular washout of PE. 


\section{Equations for ligand trapping model variations:}

Model with competitive inhibition with prazosin

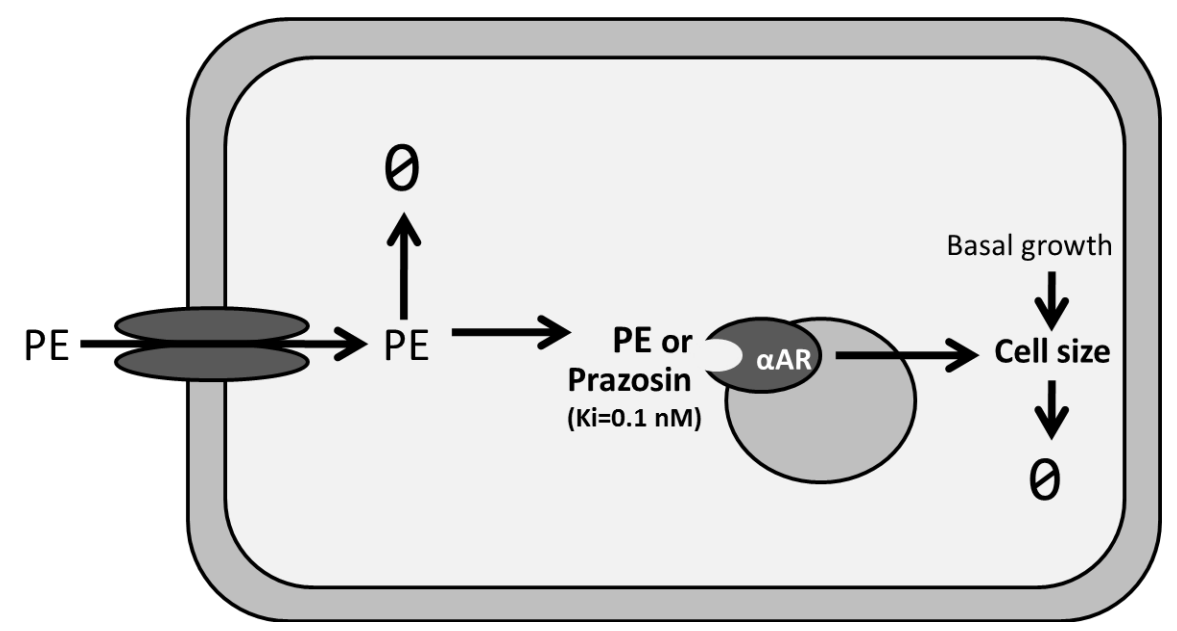

After PE washout at 24 hours:

$\frac{d \text { CellSize }}{d t}=\frac{k 3 * P E_{\text {in }}}{K d\left(1+\frac{I}{K i}\right)+P E_{\text {in }}}-k 4 *$ CellSize $+k 5$

$\mathrm{I}=10 \mu \mathrm{mol} / \mathrm{L}$

$\mathrm{Ki}=0.1 \mathrm{nM}$

Model with only sarcolemmal $\alpha A R^{\prime} s$

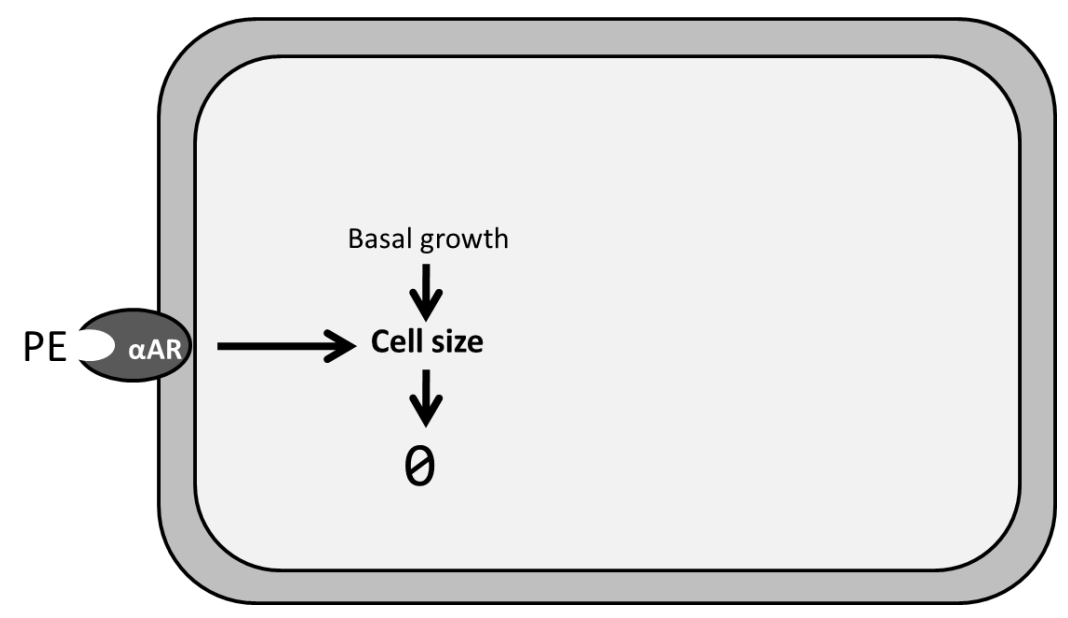

Cell size is affected by the extracellular PE concentration, $\mathrm{PE}_{\text {out, }}$ instead of the intracellular PE concentration. Therefore, after extracellular PE is washed out, cell size decreases for all conditions.

$\frac{d \text { CellSize }}{d t}=\frac{k 3 * P E_{\text {out }}}{K d+P E_{\text {out }}}-k 4 *$ CellSize $+k 5$ 


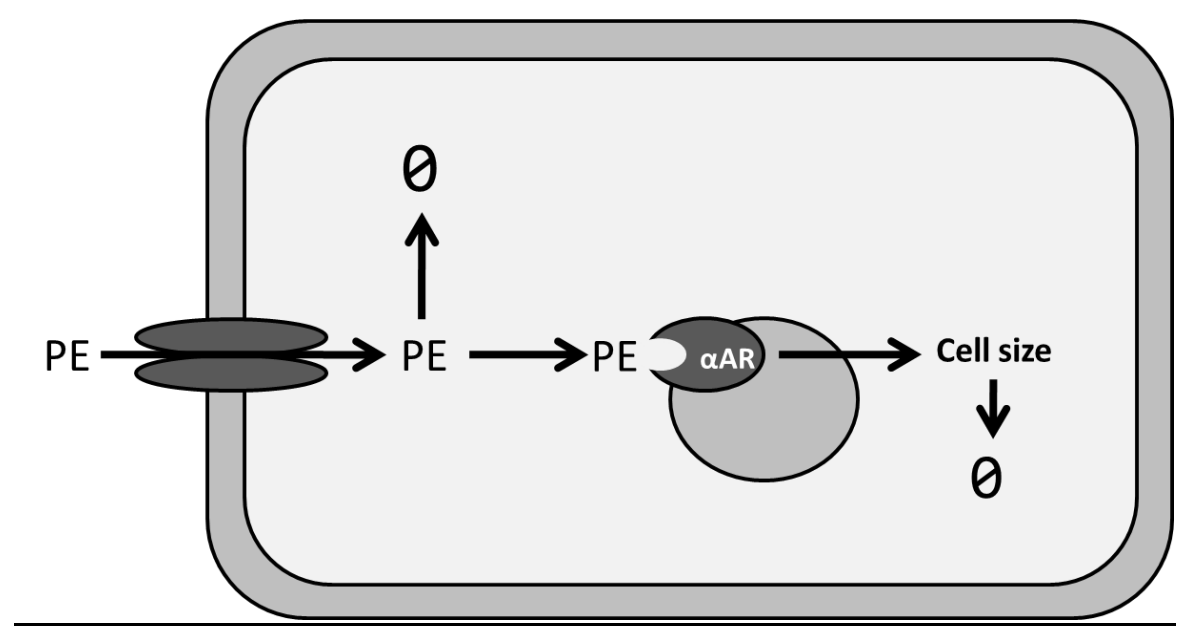

In the model formulation without the basal growth rate, $\mathrm{k} 5$, cell size cannot decrease below its original value. This is not consistent with experimental observations.

$$
\frac{d P E_{\text {in }}}{d t}=k 1 * P E_{\text {out }}-\frac{k 2 * P E_{\text {in }}}{K d 2+P E_{\text {in }}}
$$

$\frac{d \text { CellGrowth }}{d t}=\frac{k 3 * P E_{\text {in }}}{K d+P E_{\text {in }}}-k 4 *$ CellGrowth

$$
\text { CellSize }=\text { CellGrowth }+1
$$

\section{Model without saturation of PE degradation}

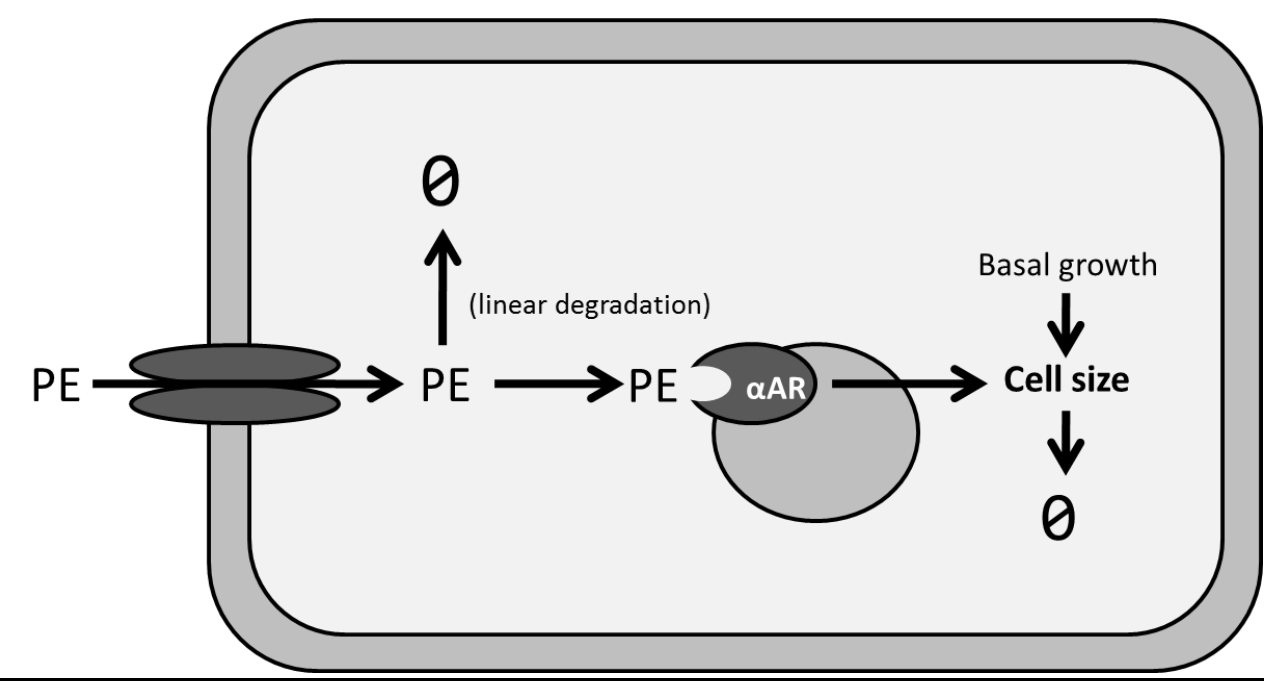

In this model formulation, the degradation term for intracellular $P E, P E_{i n}$, is linear instead of saturating. 


$$
\begin{aligned}
& \frac{d P E_{\text {in }}}{d t}=k 1 * P E_{\text {out }}-k 2 * P E_{\text {in }} \\
& \frac{d \text { CellSize }}{d t}=\frac{k 3 * P E_{\text {in }}}{K d+P E_{\text {in }}}-k 4 * \text { CellSize }+k 5
\end{aligned}
$$

The following parameters were fit to the data in Figure 2a:

$\mathrm{k} 1=0.2447$ hours $^{-1}$

$\mathrm{k} 2=0.2965$ hours $^{-1}$

$\mathrm{k} 3=0.0171$ hours $^{-1}$

$\mathrm{k} 4=0.010$ hours $^{-1}$

k5 $=.008 \frac{\mu \text { mol }}{\mathrm{L} * \text { hours }}$

$\mathrm{Kd}=1.8979 \mu \mathrm{mol} / \mathrm{L}$ 


\section{Appendix C}

Hypertrophy signaling network model species and reaction list 


\section{Hypertrophy signaling network species and reactions}

\section{Species:}

The species name, abbreviation, initial value (Yinit), maximum value (Ymax), and tau are listed for each species.

\begin{tabular}{|c|c|c|c|c|}
\hline ID & name & Yinit & Ymax & tau \\
\hline $\mathrm{aAR}$ & alpha_adrenergicReceptor & 0 & 1 & 1 \\
\hline$A C$ & AdenylyICyclase & 0 & 1 & 1 \\
\hline Akt & Protein Kinase B & 0 & 1 & 1 \\
\hline aMHC & alphaMyosinHeavyChain & 1 & 1 & 1 \\
\hline Angll & Angiotensin II & 0 & 1 & 1 \\
\hline ANP & AtrialNatriuretic Factor & 0 & 1 & 1 \\
\hline ANPi & AtrialNatriureticFactor Input & 0 & 1 & 1 \\
\hline AT1R & Angiotensin II receptor & 0 & 1 & 1 \\
\hline ATF2 & Activating Transcription Factor 2 & 0 & 1 & 1 \\
\hline BAR & B-adrenergic Receptor & 0 & 1 & 1 \\
\hline $\mathrm{bMHC}$ & beta Myosin Heavy Chain & 0 & 1 & 1 \\
\hline BNP & Brain Natriuretic Peptide & 0 & 1 & 1 \\
\hline BNPi & Brain Natriuretic Peptide Input & 0 & 1 & 1 \\
\hline Calcium & Calcium & 0 & 1 & 1 \\
\hline CaM & Calmodulin & 0 & 1 & 1 \\
\hline CaMK & CaM Kinase & 0 & 1 & 1 \\
\hline CAMP & cyclic AMP & 0 & 1 & 1 \\
\hline CaN & Calcineurin & 0 & 1 & 1 \\
\hline CellArea & Increased Cell Area & 0 & 1 & 1 \\
\hline cFos & Protein c-Fos & 0 & 1 & 1 \\
\hline cGMP & cyclic Guanosine Monophosphate & 0 & 1 & 1 \\
\hline cJun & Protein cJun & 0 & 1 & 1 \\
\hline CREB & cAMP Response Element Binding & 0 & 1 & 1 \\
\hline CT1 & Cardiotrophin-1 & 0 & 1 & 1 \\
\hline DAG & Diacylglycerol & 0 & 1 & 1 \\
\hline EGF & Epidermal Growth Factor & 0 & 1 & 1 \\
\hline EGFR & Epidermal Growth Factor Receptor & 0 & 1 & 1 \\
\hline elF2B & Eukaryotic Initiation Factor 2 & 0 & 1 & 1 \\
\hline elF4E & Eukaryotic translation initiation factor $4 \mathrm{E}$ & 0 & 1 & 1 \\
\hline ELK1 & ELK1 & 0 & 1 & 1 \\
\hline ERBB & $\begin{array}{l}\text { Erythroblastic leukemia viral oncogene homolog } \\
2 \text { and } 3 \text { or } 4\end{array}$ & 0 & 1 & 1 \\
\hline ERK12 & Extracellular signal-regulated kinases 1 or 2 & 0 & 1 & 1 \\
\hline ERK5 & Erk5 & 0 & 1 & 1 \\
\hline
\end{tabular}




\begin{tabular}{|c|c|c|c|c|}
\hline ET1 & Endothelin-1 & 0 & 1 & 1 \\
\hline ET1R & Endothelin-1 receptor & 0 & 1 & 1 \\
\hline FAK & Focal Adhesion Kinase & 0 & 1 & 1 \\
\hline FGF & Fibroblast Growth Factor & 0 & 1 & 1 \\
\hline FGFR & Fibroblast Growth Factor Receptor & 0 & 1 & 1 \\
\hline foxo & Forkhead Box O & 1 & 1 & 1 \\
\hline Gaq11 & G protein alpha subunit q or 11 & 0 & 1 & 1 \\
\hline GATA4 & Protein GATA & 0 & 1 & 1 \\
\hline GBG & G protein beta and gamma subunits & 0 & 1 & 1 \\
\hline GCA & Guanylate Cyclase A & 0 & 1 & 1 \\
\hline gp130LIFR & $\begin{array}{l}\text { Leukemia inhibitory factor receptor alpha and } \\
\text { interleukin } 6 \text { signal transducer gp } 130\end{array}$ & 0 & 1 & 1 \\
\hline Gsa & G protein alpha s & 0 & 1 & 1 \\
\hline GSK3B & Glycogen synthase kinase 3 beta & 1 & 1 & 1 \\
\hline HDAC & Histone deacetylase & 1 & 1 & 1 \\
\hline IGF1 & Insulin-like Growth Factor 1 & 0 & 1 & 1 \\
\hline IGF1R & Insulin-like Growth Factor 1 Receptor & 0 & 1 & 1 \\
\hline IkB & $\begin{array}{l}\text { Nuclear factor of kappa light polypeptide gene } \\
\text { enhancer in B-cells inhibitor, alpha }\end{array}$ & 1 & 1 & 1 \\
\hline IKK & $\begin{array}{l}\text { Inhibitor of kappa light polypeptide gene } \\
\text { enhancer in B-cells, kinase beta }\end{array}$ & 0 & 1 & 1 \\
\hline IL6 & Interleukin 6 & 0 & 1 & 1 \\
\hline IL6R & $\begin{array}{l}\text { Interleukin } 6 \text { Receptor and interleukin } 6 \text { signal } \\
\text { transducer gp130 }\end{array}$ & 0 & 1 & 1 \\
\hline Integrins & Integrins & 0 & 1 & 1 \\
\hline IP3 & Inositol Triphosphate & 0 & 1 & 1 \\
\hline ISO & Isoproterenol & 0 & 1 & 1 \\
\hline JAK & Janus kinase 1 or 2 & 0 & 1 & 1 \\
\hline JNK & c-Jun N-terminal kinase & 0 & 1 & 1 \\
\hline LIF & Leukemia inhibitory factor & 0 & 1 & 1 \\
\hline MAP3K11 & MAPK Kinase Kinase 11 & 0 & 1 & 1 \\
\hline MAP3K23 & MAPK Kinase Kinase 2 or 3 & 0 & 1 & 1 \\
\hline MAP3K4 & MAPK Kinase Kinase 4 & 0 & 1 & 1 \\
\hline MAPKAPK & MAPK-Activated Protein Kinase & 0 & 1 & 1 \\
\hline MEF2 & Myocyte Enhancer Factor-2 & 0 & 1 & 1 \\
\hline MEK12 & MAPK kinase 1 or MAPK kinase 2 & 0 & 1 & 1 \\
\hline MEK36 & MAPK kinase 3 or MAPK kinase 6 & 0 & 1 & 1 \\
\hline MEK4 & MAPK Kinase 4 & 0 & 1 & 1 \\
\hline MEK5 & MAPK kinase 5 & 0 & 1 & 1 \\
\hline MEK7 & MAPK Kinase 7 & 0 & 1 & 1 \\
\hline MEKK1 & MAPK Kinase Kinase 1 & 0 & 1 & 1 \\
\hline
\end{tabular}




\begin{tabular}{|c|c|c|c|c|}
\hline MSK1 & $\begin{array}{l}\text { ribosomal protein } 56 \text { kinase, } 90 \mathrm{kDa} \text {, polypeptide } \\
5\end{array}$ & 0 & 1 & 1 \\
\hline mTor & mechanistic Target Of Rapamycin & 0 & 1 & 1 \\
\hline NE & Norepinephrine & 0 & 1 & 1 \\
\hline NFAT & Nuclear Factor of Activated T-cells & 0 & 1 & 1 \\
\hline NFkB & $\begin{array}{l}\text { Nuclear Factor kappa-light-chain-enhancer of } \\
\text { activated B cells }\end{array}$ & 0 & 1 & 1 \\
\hline NIK & NFkB Inducing Kinase & 0 & 1 & 1 \\
\hline NOS & Endothelial Nitric Oxide Synthase & 0 & 1 & 1 \\
\hline NRG1 & Neuregulin 1 & 0 & 1 & 1 \\
\hline p38 & P38 mitogen-activated protein kinase & 0 & 1 & 1 \\
\hline p70s6k & $70 \mathrm{kDa}$ ribosomal protein $\mathrm{S6}$ kinase I & 0 & 1 & 1 \\
\hline PDK1 & 3-phosphoinositide dependent protein kinase-1 & 0 & 1 & 1 \\
\hline $\mathrm{PE}$ & Phenylephrine & 0 & 1 & 1 \\
\hline PI3K & Phosphatidyl Inositol 3 Kinase & 0 & 1 & 1 \\
\hline PKA & Protein Kinase $\mathrm{A}$ & 0 & 1 & 1 \\
\hline PKC & Protein Kinase $\mathrm{C}$ & 0 & 1 & 1 \\
\hline PKD & Protein Kinase D & 0 & 1 & 1 \\
\hline PKG1 & cGMP-dependent protein kinase 1 & 0 & 1 & 1 \\
\hline PLCB & Phospholipase C Beta & 0 & 1 & 1 \\
\hline PLCG & Phopholipase C Gamma 1 & 0 & 1 & 1 \\
\hline Rac1 & Ras-related C 3 botulinum toxin substrate 1 & 0 & 1 & 1 \\
\hline Raf1 & Raf1 & 0 & 1 & 1 \\
\hline Raf1A & Activated Raf1 & 0 & 1 & 1 \\
\hline Ras & Rat sarcoma viral oncogene homolog & 0 & 1 & 1 \\
\hline RhoA & Ras homolog gene family, member A & 0 & 1 & 1 \\
\hline sACT & Alpha Skeletal Actin & 0 & 1 & 1 \\
\hline SERCA & Sarcoplastic Reticulum Ca2+ ATPase & 1 & 1 & 1 \\
\hline sGC & soluble Guanylyl Cyclase & 0 & 1 & 1 \\
\hline SHP2 & $\begin{array}{l}\text { Protein tyrosine phosphatase, non-receptor } \\
\text { type } 11\end{array}$ & 0 & 1 & 1 \\
\hline SRF & Serum Response Factor & 0 & 1 & 1 \\
\hline STAT & $\begin{array}{l}\text { Signal Transducers and Activators of } \\
\text { Transcription }\end{array}$ & 0 & 1 & 1 \\
\hline Stretch & Stretch & 0 & 1 & 1 \\
\hline TAK1 & TGF-beta Activated Kinase 1 & 0 & 1 & 1 \\
\hline TGFB & Transforming Growth Factor beta & 0 & 1 & 1 \\
\hline TGFR & Transforming Growth Factor Receptors & 0 & 1 & 1 \\
\hline TNFa & Tumor Necrosis Factor-alpha & 0 & 1 & 1 \\
\hline TNFR & Tumor Necrosis Factor Receptor & 0 & 1 & 1 \\
\hline
\end{tabular}




\section{Reactions:}

Each reaction included in the network model is specified below. A "!" indicates inhibition and ' $\&$ ' indicates an AND reaction. When multiple reactions affect a species through an OR relationship, those reactions are listed in separate rows. Parameter values are also provided for each reaction. We used default parameters of weight $=1, \mathrm{n}=1.4$, and $\mathrm{EC} 59=0.5$.

\begin{tabular}{|c|c|c|c|c|}
\hline ID & Rule & Weight & $\underline{\mathrm{n}}$ & EC50 \\
\hline r1 & $\Rightarrow$ Angll & 0 & 1.4 & 0.5 \\
\hline$r 2$ & $\Rightarrow$ ANPi & 0 & 1.4 & 0.5 \\
\hline r3 & $\Rightarrow \mathrm{BNPi}$ & 0 & 1.4 & 0.5 \\
\hline r4 & $\Rightarrow$ CT1 & 0 & 1.4 & 0.5 \\
\hline r5 & $\Rightarrow$ EGF & 0 & 1.4 & 0.5 \\
\hline r6 & $=>$ ET1 & 0 & 1.4 & 0.5 \\
\hline$r 7$ & $\Rightarrow>F G F$ & 0 & 1.4 & 0.5 \\
\hline r8 & $\Rightarrow>$ IGF1 & 0 & 1.4 & 0.5 \\
\hline r9 & $\Rightarrow>$ IL6 & 0 & 1.4 & 0.5 \\
\hline r10 & $=>$ ISO & 0 & 1.4 & 0.5 \\
\hline r11 & $\Rightarrow$ LIF & 0 & 1.4 & 0.5 \\
\hline r12 & $\Rightarrow \mathrm{NE}$ & 0 & 1.4 & 0.5 \\
\hline r13 & $\Rightarrow>N R G 1$ & 0 & 1.4 & 0.5 \\
\hline r14 & $\Rightarrow P E$ & 0 & 1.4 & 0.5 \\
\hline r15 & $\Rightarrow>$ Stretch & 0 & 1.4 & 0.5 \\
\hline r16 & $\Rightarrow>$ TGFB & 0 & 1.4 & 0.5 \\
\hline r17 & $\Rightarrow$ TNFa & 0 & 1.4 & 0.5 \\
\hline r18 & $!$ Akt $=>$ foxo & 1 & 1.4 & 0.5 \\
\hline r19 & $! \mathrm{Akt}=>$ GSK3B & 1 & 1.4 & 0.5 \\
\hline r20 & $! \mathrm{CaMK}=>\mathrm{HDAC}$ & 1 & 1.4 & 0.5 \\
\hline r21 & !cFos \& !cJun \& !MEF2 \& !NFAT $=>$ aMHC & 1 & 1.4 & 0.5 \\
\hline r22 & !cFos \& !cJun \& !NFAT $=>$ SERCA & 1 & 1.4 & 0.5 \\
\hline r23 & !foxo => CellArea & 1 & 1.4 & 0.5 \\
\hline r24 & $\begin{array}{l}\text { !GSK3B \& !JNK \& !p38 \& !PKG1 \& !PKA => } \\
\text { NFAT }\end{array}$ & 1 & 1.4 & 0.5 \\
\hline r25 & !GSK3B $=>$ CREB & 1 & 1.4 & 0.5 \\
\hline r26 & !GSK3B => elF2B & 1 & 1.4 & 0.5 \\
\hline r27 & !GSK3B => GATA4 & 1 & 1.4 & 0.5 \\
\hline r28 & !HDAC $=>$ MEF2 & 1 & 1.4 & 0.5 \\
\hline r29 & $! \mid k B=>N F k B$ & 1 & 1.4 & 0.5 \\
\hline r30 & $! I K K=>I k B$ & 1 & 1.4 & 0.5 \\
\hline r31 & !PKA \& Raf1A => Raf1 & 1 & 1.4 & 0.5 \\
\hline r32 & $! P K C=>$ HDAC & 1 & 1.4 & 0.5 \\
\hline r33 & $! P K D=>$ HDAC & 1 & 1.4 & 0.5 \\
\hline r34 & $\mathrm{aAR}=>\mathrm{Gaq} 11$ & 1 & 1.4 & 0.5 \\
\hline
\end{tabular}




\begin{tabular}{|c|c|c|c|c|}
\hline r35 & $A C=>c A M P$ & 1 & 1.4 & 0.5 \\
\hline r36 & $A k t=>I K K$ & 1 & 1.4 & 0.5 \\
\hline r37 & Akt $=>\mathrm{mTor}$ & 1 & 1.4 & 0.5 \\
\hline r38 & Akt $=>$ NOS & 1 & 1.4 & 0.5 \\
\hline r39 & Angll $=>$ AT1R & 1 & 1.4 & 0.5 \\
\hline $\mathrm{r} 40$ & ANPi $=>$ GCA & 1 & 1.4 & 0.5 \\
\hline r41 & AT1R $=>$ Gaq11 & 1 & 1.4 & 0.5 \\
\hline r42 & AT1R $=>$ JAK & 1 & 1.4 & 0.5 \\
\hline r43 & ATF2 $\Rightarrow>$ ANP & 1 & 1.4 & 0.5 \\
\hline r44 & ATF2 $=>$ bMHC & 1 & 1.4 & 0.5 \\
\hline r45 & ATF2 $=>$ BNP & 1 & 1.4 & 0.5 \\
\hline r46 & ATF2 $=>$ CellArea & 1 & 1.4 & 0.5 \\
\hline r47 & BAR $=>$ Gsa & 1 & 1.4 & 0.5 \\
\hline r48 & BNPi $=>$ GCA & 1 & 1.4 & 0.5 \\
\hline r49 & Calcium $=>$ CaM & 1 & 1.4 & 0.5 \\
\hline r50 & CaM $=>$ CaMK & 1 & 1.4 & 0.5 \\
\hline r51 & $\mathrm{CaM}=>\mathrm{CaN}$ & 1 & 1.4 & 0.5 \\
\hline r52 & CAMP $=>$ PKA & 1 & 1.4 & 0.5 \\
\hline r53 & CaN $=>$ NFAT & 1 & 1.4 & 0.5 \\
\hline r54 & cFos \& cJun \& SRF $=>$ SACT & 1 & 1.4 & 0.5 \\
\hline r55 & cFos $=>$ ANP & 1 & 1.4 & 0.5 \\
\hline r56 & cFos $=>$ bMHC & 1 & 1.4 & 0.5 \\
\hline r57 & cFos $=>$ BNP & 1 & 1.4 & 0.5 \\
\hline r58 & cGMP $=>$ PKG1 & 1 & 1.4 & 0.5 \\
\hline r59 & cJun $\&$ SRF $=>$ SACT & 1 & 1.4 & 0.5 \\
\hline r60 & cJun $=>$ ANP & 1 & 1.4 & 0.5 \\
\hline r61 & cJun $=>$ bMHC & 1 & 1.4 & 0.5 \\
\hline r62 & cJun $=>$ BNP & 1 & 1.4 & 0.5 \\
\hline r63 & cJun $=>$ CellArea & 1 & 1.4 & 0.5 \\
\hline r64 & CREB $=>$ ANP & 1 & 1.4 & 0.5 \\
\hline r65 & CREB $=>$ CellArea & 1 & 1.4 & 0.5 \\
\hline r66 & CT1 => gp130LIFR & 1 & 1.4 & 0.5 \\
\hline r67 & DAG \& Calcium $=>$ PKC & 1 & 1.4 & 0.5 \\
\hline r68 & $E G F=>E G F R$ & 1 & 1.4 & 0.5 \\
\hline r69 & $E G F R=>P I 3 K$ & 1 & 1.4 & 0.5 \\
\hline r70 & $E G F R=>P L C G$ & 1 & 1.4 & 0.5 \\
\hline$r 71$ & EGFR $=>$ Ras & 1 & 1.4 & 0.5 \\
\hline r72 & ELK1 $=>$ BNP & 1 & 1.4 & 0.5 \\
\hline r73 & $E R B B=>P I 3 K$ & 1 & 1.4 & 0.5 \\
\hline r74 & $E R B B=>P L C G$ & 1 & 1.4 & 0.5 \\
\hline r75 & $E R B B=>$ Ras & 1 & 1.4 & 0.5 \\
\hline
\end{tabular}




\begin{tabular}{|c|c|c|c|c|}
\hline r76 & ERK12 \& CaN $=>$ NFAT & 1 & 1.4 & 0.5 \\
\hline$r 77$ & ERK12 $=>$ cFos & 1 & 1.4 & 0.5 \\
\hline$r 78$ & ERK12 $=>$ cJun & 1 & 1.4 & 0.5 \\
\hline$r 79$ & ERK12 => ELK1 & 1 & 1.4 & 0.5 \\
\hline r80 & ERK12 => GATA4 & 1 & 1.4 & 0.5 \\
\hline r81 & ERK12 => MSK1 & 1 & 1.4 & 0.5 \\
\hline r82 & $\mathrm{ERK} 12=>\mathrm{NFkB}$ & 1 & 1.4 & 0.5 \\
\hline r83 & ERK5 => MEF2 & 1 & 1.4 & 0.5 \\
\hline r84 & ET1 $=>$ ET1R & 1 & 1.4 & 0.5 \\
\hline r85 & ET1R => Gaq11 & 1 & 1.4 & 0.5 \\
\hline r86 & FAK $=>$ Ras & 1 & 1.4 & 0.5 \\
\hline r87 & $F G F=>F G F R$ & 1 & 1.4 & 0.5 \\
\hline r88 & FGFR $=>$ Ras & 1 & 1.4 & 0.5 \\
\hline r89 & Gaq11 $=>$ GBG & 1 & 1.4 & 0.5 \\
\hline r90 & Gaq11 => PLCB & 1 & 1.4 & 0.5 \\
\hline r91 & GATA4 \& SRF $=>$ SACT & 1 & 1.4 & 0.5 \\
\hline r92 & GATA4 $=>$ bMHC & 1 & 1.4 & 0.5 \\
\hline r93 & GATA4 => CellArea & 1 & 1.4 & 0.5 \\
\hline r94 & GBG $=>$ PI3K & 1 & 1.4 & 0.5 \\
\hline r95 & GBG $=>$ Raf1A & 1 & 1.4 & 0.5 \\
\hline r96 & GBG $=>$ Ras & 1 & 1.4 & 0.5 \\
\hline r97 & $\mathrm{GCA}=>\mathrm{cGMP}$ & 1 & 1.4 & 0.5 \\
\hline r98 & gp130LIFR => JAK & 1 & 1.4 & 0.5 \\
\hline r99 & gp130LIFR => SHP2 & 1 & 1.4 & 0.5 \\
\hline r100 & $\mathrm{Gsa}=>\mathrm{AC}$ & 1 & 1.4 & 0.5 \\
\hline r101 & Gsa $=>$ GBG & 1 & 1.4 & 0.5 \\
\hline r102 & IGF1 => IGF1R & 1 & 1.4 & 0.5 \\
\hline r103 & IGF1R => PI3K & 1 & 1.4 & 0.5 \\
\hline r104 & IGF1R => PLCB & 1 & 1.4 & 0.5 \\
\hline r105 & IGF1R => Ras & 1 & 1.4 & 0.5 \\
\hline r106 & IL6 => IL6R & 1 & 1.4 & 0.5 \\
\hline r107 & IL6R => JAK & 1 & 1.4 & 0.5 \\
\hline r108 & Integrins $=>\mathrm{FAK}$ & 1 & 1.4 & 0.5 \\
\hline r109 & IP3 => Calcium & 1 & 1.4 & 0.5 \\
\hline $\mathrm{r} 110$ & ISO => BAR & 1 & 1.4 & 0.5 \\
\hline r111 & $J A K=>P I 3 K$ & 1 & 1.4 & 0.5 \\
\hline$r 112$ & JAK $=>$ Ras & 1 & 1.4 & 0.5 \\
\hline $\mathrm{r} 113$ & $J A K=>$ STAT & 1 & 1.4 & 0.5 \\
\hline r114 & JNK => ATF2 & 1 & 1.4 & 0.5 \\
\hline r115 & JNK => cJun & 1 & 1.4 & 0.5 \\
\hline r116 & JNK $=>$ ELK1 & 1 & 1.4 & 0.5 \\
\hline
\end{tabular}




\begin{tabular}{|c|c|c|c|c|}
\hline r117 & LIF => gp130LIFR & 1 & 1.4 & 0.5 \\
\hline r118 & MAP3K11 => MEK36 & 1 & 1.4 & 0.5 \\
\hline r119 & MAP3K11 => MEK7 & 1 & 1.4 & 0.5 \\
\hline r120 & MAP3K23 => MEK12 & 1 & 1.4 & 0.5 \\
\hline r121 & MAP3K23 => MEK4 & 1 & 1.4 & 0.5 \\
\hline r122 & MAP3K23 => MEK5 & 1 & 1.4 & 0.5 \\
\hline r123 & MAP3K23 => MEK7 & 1 & 1.4 & 0.5 \\
\hline r124 & MAP3K4 => MEK4 & 1 & 1.4 & 0.5 \\
\hline r125 & MAP3K4 => MEK7 & 1 & 1.4 & 0.5 \\
\hline r126 & MAPKAPK $=>$ CREB & 1 & 1.4 & 0.5 \\
\hline r127 & MEF2 $=>$ ANP & 1 & 1.4 & 0.5 \\
\hline r128 & $\mathrm{MEF} 2=>\mathrm{bMHC}$ & 1 & 1.4 & 0.5 \\
\hline r129 & MEF2 $=>$ BNP & 1 & 1.4 & 0.5 \\
\hline r130 & MEF2 => CellArea & 1 & 1.4 & 0.5 \\
\hline r131 & $\mathrm{MEF} 2=>\mathrm{sACT}$ & 1 & 1.4 & 0.5 \\
\hline r132 & MEK12 => ERK12 & 1 & 1.4 & 0.5 \\
\hline r133 & MEK36 => p38 & 1 & 1.4 & 0.5 \\
\hline r134 & MEK4 => JNK & 1 & 1.4 & 0.5 \\
\hline r135 & MEK4 => p38 & 1 & 1.4 & 0.5 \\
\hline r136 & MEK5 => ERK5 & 1 & 1.4 & 0.5 \\
\hline r137 & MEK7 => JNK & 1 & 1.4 & 0.5 \\
\hline$r 138$ & MEKK1 => MEK12 & 1 & 1.4 & 0.5 \\
\hline r139 & MEKK1 => MEK4 & 1 & 1.4 & 0.5 \\
\hline r140 & MEKK1 => MEK7 & 1 & 1.4 & 0.5 \\
\hline r141 & MSK1 => CREB & 1 & 1.4 & 0.5 \\
\hline r142 & $\mathrm{mTor}=>$ elF4E & 1 & 1.4 & 0.5 \\
\hline r143 & $\mathrm{mTor}=>\mathrm{p} 70 \mathrm{~s} 6 \mathrm{k}$ & 1 & 1.4 & 0.5 \\
\hline r144 & $\mathrm{NE}=>\mathrm{aAR}$ & 1 & 1.4 & 0.5 \\
\hline r145 & $N E=>B A R$ & 1 & 1.4 & 0.5 \\
\hline r146 & NFAT \& GATA4 => ANP & 1 & 1.4 & 0.5 \\
\hline r147 & NFAT \& GATA4 => BNP & 1 & 1.4 & 0.5 \\
\hline r148 & NFAT $=>$ bMHC & 1 & 1.4 & 0.5 \\
\hline r149 & NFAT $=>$ sACT & 1 & 1.4 & 0.5 \\
\hline r150 & NIK => IKK & 1 & 1.4 & 0.5 \\
\hline r151 & $\mathrm{NOS}=>\mathrm{sGC}$ & 1 & 1.4 & 0.5 \\
\hline r152 & NRG1 $=>$ ERBB & 1 & 1.4 & 0.5 \\
\hline r153 & p38 => ATF2 & 1 & 1.4 & 0.5 \\
\hline r154 & p38 => ELK1 & 1 & 1.4 & 0.5 \\
\hline r155 & p38 => GATA4 & 1 & 1.4 & 0.5 \\
\hline r156 & $\mathrm{p} 38=>\mathrm{IKK}$ & 1 & 1.4 & 0.5 \\
\hline r157 & p38 => MAPKAPK & 1 & 1.4 & 0.5 \\
\hline
\end{tabular}




\begin{tabular}{|c|c|c|c|c|}
\hline r158 & p38 => MEF2 & 1 & 1.4 & 0.5 \\
\hline r159 & p38 => MSK1 & 1 & 1.4 & 0.5 \\
\hline r160 & PDK1 $=>$ Akt & 1 & 1.4 & 0.5 \\
\hline r161 & $P E=>a A R$ & 1 & 1.4 & 0.5 \\
\hline r162 & PI3K => PDK1 & 1 & 1.4 & 0.5 \\
\hline r163 & PKA $=>$ Calcium & 1 & 1.4 & 0.5 \\
\hline r164 & PKA $=>$ CREB & 1 & 1.4 & 0.5 \\
\hline r165 & $\mathrm{PKC}=>\mathrm{PKD}$ & 1 & 1.4 & 0.5 \\
\hline r166 & $\mathrm{PKC}=>$ Raf1A & 1 & 1.4 & 0.5 \\
\hline r167 & $\mathrm{PKC}=>$ Ras & 1 & 1.4 & 0.5 \\
\hline r168 & $\mathrm{PKC}=>\mathrm{TAK} 1$ & 1 & 1.4 & 0.5 \\
\hline r169 & $P L C B=>D A G$ & 1 & 1.4 & 0.5 \\
\hline r170 & PLCB $=>$ IP3 & 1 & 1.4 & 0.5 \\
\hline r171 & PLCG $=>$ DAG & 1 & 1.4 & 0.5 \\
\hline$r 172$ & PLCG $=>$ IP3 & 1 & 1.4 & 0.5 \\
\hline r173 & Rac1 => MAP3K11 & 1 & 1.4 & 0.5 \\
\hline r174 & Rac1 => MAP3K4 & 1 & 1.4 & 0.5 \\
\hline r175 & Raf1 => MEK12 & 1 & 1.4 & 0.5 \\
\hline r176 & Ras \& !SHP2 => RhoA & 1 & 1.4 & 0.5 \\
\hline r177 & Ras => MAP3K23 & 1 & 1.4 & 0.5 \\
\hline r178 & Ras => MEKK1 & 1 & 1.4 & 0.5 \\
\hline r179 & Ras $=>\mathrm{PI} 3 \mathrm{~K}$ & 1 & 1.4 & 0.5 \\
\hline $\mathrm{r} 180$ & Ras $=>$ Rac1 & 1 & 1.4 & 0.5 \\
\hline r181 & Ras $=>$ Raf1A & 1 & 1.4 & 0.5 \\
\hline r182 & RhoA => SRF & 1 & 1.4 & 0.5 \\
\hline r183 & $s G C=>c G M P$ & 1 & 1.4 & 0.5 \\
\hline r184 & SHP2 => MEK5 & 1 & 1.4 & 0.5 \\
\hline r185 & STAT $=>$ ANP & 1 & 1.4 & 0.5 \\
\hline r186 & STAT $=>$ bMHC & 1 & 1.4 & 0.5 \\
\hline r187 & Stretch $=>$ Integrins & 1 & 1.4 & 0.5 \\
\hline r188 & TAK1 => MEK36 & 1 & 1.4 & 0.5 \\
\hline r189 & TGFB $=>$ TGFR & 1 & 1.4 & 0.5 \\
\hline r190 & TGFR $=>$ PKC & 1 & 1.4 & 0.5 \\
\hline r191 & TNFa $=>$ TNFR & 1 & 1.4 & 0.5 \\
\hline r192 & TNFR $=>$ NIK & 1 & 1.4 & 0.5 \\
\hline r193 & TNFR => PI3K & 1 & 1.4 & 0.5 \\
\hline
\end{tabular}




\section{Appendix D}

\section{References for experimental validation of transcription factor activities and phenotypic outputs}


Pubmed ID numbers for the experimental data used to validate model predicted input-output relationships are provided below.

\begin{tabular}{|c|c|c|c|c|c|c|}
\hline Input 1 & Input 2 & Output & Prediction & Measurement & Reference & Additional References \\
\hline Angll & & $\mathrm{aMHC}$ & Decrease & No Change & 17111039 & 8946229 \\
\hline Angll & & ANP & Increase & Increase & 8348686 & $\begin{array}{l}9396478,11714734,11815436, \\
11889020\end{array}$ \\
\hline Angll & & bMHC & Increase & Increase & 11322781 & $8946229,17111039,19713680$ \\
\hline Angll & & BNP & Increase & Increase & 9396478 & \\
\hline Angll & & CellArea & Increase & Increase & 9396478 & $\begin{array}{l}11714734,11815436,9727550, \\
19713680\end{array}$ \\
\hline Angll & & SACT & Increase & Increase & 8348686 & 11889020 \\
\hline Angll & & SERCA & Decrease & Decrease & 8762048 & \\
\hline ANP & Angll & $\mathrm{aMHC}$ & $\begin{array}{l}\text { No } \\
\text { Change }\end{array}$ & No Data & & \\
\hline ANP & Angll & ANP & $\begin{array}{l}\text { No } \\
\text { Change }\end{array}$ & Decrease & 15313208 & \\
\hline ANP & Angll & $\mathrm{bMHC}$ & $\begin{array}{l}\text { No } \\
\text { Change }\end{array}$ & Decrease & 16890211 & \\
\hline ANP & Angll & BNP & $\begin{array}{l}\text { No } \\
\text { Change }\end{array}$ & Decrease & 15313208 & \\
\hline ANP & Angll & CellArea & $\begin{array}{l}\text { No } \\
\text { Change }\end{array}$ & Decrease & 15313208 & 16890211 \\
\hline ANP & Angll & sACT & $\begin{array}{l}\text { No } \\
\text { Change }\end{array}$ & No Data & & \\
\hline ANP & Angll & SERCA & $\begin{array}{l}\text { No } \\
\text { Change }\end{array}$ & No Data & & \\
\hline ANP & ET1 & $\mathrm{aMHC}$ & $\begin{array}{l}\text { No } \\
\text { Change }\end{array}$ & No Data & & \\
\hline ANP & ET1 & ANP & $\begin{array}{l}\text { No } \\
\text { Change }\end{array}$ & Decrease & 15313208 & \\
\hline ANP & ET1 & $\mathrm{bMHC}$ & $\begin{array}{l}\text { No } \\
\text { Change }\end{array}$ & No Data & & \\
\hline ANP & ET1 & BNP & $\begin{array}{l}\text { No } \\
\text { Change }\end{array}$ & Decrease & 15313208 & \\
\hline ANP & ET1 & CellArea & $\begin{array}{l}\text { No } \\
\text { Change }\end{array}$ & Decrease & 15313208 & 16890211 \\
\hline ANP & ET1 & sACT & $\begin{array}{l}\text { No } \\
\text { Change }\end{array}$ & No Data & & \\
\hline ANP & ET1 & SERCA & $\begin{array}{l}\text { No } \\
\text { Change }\end{array}$ & No Data & & \\
\hline ANP & PE & $\mathrm{aMHC}$ & $\begin{array}{l}\text { No } \\
\text { Change }\end{array}$ & No Data & & \\
\hline ANP & PE & ANP & $\begin{array}{l}\text { No } \\
\text { Change }\end{array}$ & No Data & & \\
\hline ANP & $\mathrm{PE}$ & bMHC & No & No Data & & \\
\hline
\end{tabular}




\begin{tabular}{|c|c|c|c|c|c|c|}
\hline & & & Change & & & \\
\hline ANP & PE & BNP & $\begin{array}{l}\text { No } \\
\text { Change }\end{array}$ & No Data & & \\
\hline ANP & PE & CellArea & $\begin{array}{l}\text { No } \\
\text { Change }\end{array}$ & Decrease & 10455158 & \\
\hline ANP & PE & sACT & $\begin{array}{l}\text { No } \\
\text { Change }\end{array}$ & No Data & & \\
\hline ANP & PE & SERCA & $\begin{array}{l}\text { No } \\
\text { Change }\end{array}$ & No Data & & \\
\hline ANP & & $\mathrm{aMHC}$ & $\begin{array}{l}\text { No } \\
\text { Change }\end{array}$ & No Data & & \\
\hline ANP & & ANP & $\begin{array}{l}\text { No } \\
\text { Change }\end{array}$ & No Data & & \\
\hline ANP & & $\mathrm{bMHC}$ & $\begin{array}{l}\text { No } \\
\text { Change }\end{array}$ & No Change & 16890211 & \\
\hline ANP & & BNP & $\begin{array}{l}\text { No } \\
\text { Change }\end{array}$ & No Change & 15313208 & \\
\hline ANP & & CellArea & $\begin{array}{l}\text { No } \\
\text { Change }\end{array}$ & No Change & 10455158 & 16890211,15313208 \\
\hline ANP & & SACT & $\begin{array}{l}\text { No } \\
\text { Change }\end{array}$ & No Data & & \\
\hline ANP & & SERCA & $\begin{array}{l}\text { No } \\
\text { Change }\end{array}$ & No Data & & \\
\hline BNP & & aMHC & $\begin{array}{l}\text { No } \\
\text { Change }\end{array}$ & No Data & & \\
\hline BNP & & ANP & $\begin{array}{l}\text { No } \\
\text { Change }\end{array}$ & No Data & & \\
\hline BNP & & $\mathrm{bMHC}$ & $\begin{array}{l}\text { No } \\
\text { Change }\end{array}$ & No Data & & \\
\hline BNP & & BNP & $\begin{array}{l}\text { No } \\
\text { Change }\end{array}$ & No Data & & \\
\hline BNP & & CellArea & $\begin{array}{l}\text { No } \\
\text { Change }\end{array}$ & No Data & & \\
\hline BNP & & SACT & $\begin{array}{l}\text { No } \\
\text { Change }\end{array}$ & No Data & & \\
\hline BNP & & SERCA & $\begin{array}{l}\text { No } \\
\text { Change }\end{array}$ & No Data & & \\
\hline CT1 & & $\mathrm{aMHC}$ & Decrease & No Data & & \\
\hline CT1 & & ANP & Increase & Increase & 8621626 & 7862649 \\
\hline CT1 & & $\mathrm{bMHC}$ & Increase & No Data & & \\
\hline CT1 & & BNP & Increase & Increase & 9595480 & 15623435 \\
\hline CT1 & & CellArea & Increase & Increase & 8621626 & 7862649,15623435 \\
\hline CT1 & & SACT & Increase & No Change & 8621626 & \\
\hline CT1 & & SERCA & Decrease & No Data & & \\
\hline EGF & & $\mathrm{aMHC}$ & Decrease & No Data & & \\
\hline EGF & & ANP & Increase & Increase & 11834705 & \\
\hline
\end{tabular}




\begin{tabular}{|c|c|c|c|c|c|}
\hline EGF & $\mathrm{bMHC}$ & Increase & No Data & & \\
\hline EGF & BNP & Increase & Increase & 14645255 & \\
\hline EGF & CellArea & Increase & Increase & 11834705 & \\
\hline EGF & SACT & Increase & No Data & & \\
\hline EGF & SERCA & Decrease & No Data & & \\
\hline ET1 & $\mathrm{aMHC}$ & Decrease & Increase & 1567402 & \\
\hline ET1 & ANP & Increase & Increase & 1394868 & $2531580,9396478,11815436$ \\
\hline ET1 & bMHC & Increase & Increase & 10984495 & $10809760,1567402,9428623$ \\
\hline ET1 & BNP & Increase & Increase & 1394868 & $9396478,14997707,9595480$ \\
\hline ET1 & CellArea & Increase & Increase & 2054934 & $9396478,14967736,11815436$ \\
\hline ET1 & SACT & Increase & Increase & 2054934 & 9428623,9798730 \\
\hline ET1 & SERCA & Decrease & Decrease & 14997707 & \\
\hline FGF & $\mathrm{aMHC}$ & Decrease & Decrease & 1688886 & 9428623,1712696 \\
\hline FGF & ANP & Increase & Increase & 7519562 & 9428623,1712696 \\
\hline FGF & $\mathrm{bMHC}$ & Increase & Increase & 1688886 & 9428623,1712696 \\
\hline FGF & BNP & Increase & No Data & & \\
\hline FGF & CellArea & Increase & Increase & 9428623 & \\
\hline FGF & SACT & Increase & Increase & 1688886 & $9428623,1712696,2402491$ \\
\hline FGF & SERCA & Decrease & Decrease & 9428623 & 1712696 \\
\hline IGF1 & $\mathrm{aMHC}$ & Decrease & No Data & 9428623 & \\
\hline IGF1 & ANP & Increase & Decrease & 9428623 & \\
\hline IGF1 & $\mathrm{bMHC}$ & Increase & Increase & 9428623 & \\
\hline IGF1 & BNP & Increase & No Data & & \\
\hline IGF1 & CellArea & Increase & Increase & 7683979 & 9428623 \\
\hline IGF1 & SACT & Increase & Increase & 7683979 & 9428623 \\
\hline IGF1 & SERCA & Decrease & No Data & 9428623 & \\
\hline IL6 & $\mathrm{aMHC}$ & Decrease & No Data & & \\
\hline IL6 & ANP & Increase & Increase & 14997707 & \\
\hline IL6 & $\mathrm{bMHC}$ & Increase & No Data & & \\
\hline IL6 & BNP & Increase & Increase & 14997707 & \\
\hline IL6 & CellArea & Increase & No Data & & \\
\hline IL6 & SACT & Increase & No Data & & \\
\hline IL6 & SERCA & Decrease & Decrease & 14997707 & 10752545 \\
\hline ISO & aMHC & Decrease & Decrease & 16501029 & \\
\hline ISO & ANP & Increase & Increase & 11181023 & $15001529,10799529,18194989$, \\
\hline ISO & $\mathrm{bMHC}$ & Increase & No Change & 20362664 & 16501029,18851973 \\
\hline ISO & BNP & Increase & Increase & 16501029 & \\
\hline ISO & CellArea & Increase & Increase & 6216022 & $11181023,15001529,20362664$ \\
\hline ISO & SACT & Increase & Increase & 20362664 & 16501029 \\
\hline ISO & SERCA & Decrease & Decrease & 16501029 & \\
\hline LIF & $\mathrm{aMHC}$ & Decrease & No Data & & \\
\hline
\end{tabular}




\begin{tabular}{|c|c|c|c|c|c|}
\hline LIF & ANP & Increase & Increase & 10452539 & $\begin{array}{l}\text { 11009450, 11073891, 11387209, } \\
9798730\end{array}$ \\
\hline LIF & bMHC & Increase & Increase & 10212267 & 10452539 \\
\hline LIF & BNP & Increase & Increase & 10452539 & $12855672,11009450,11073891$ \\
\hline LIF & CellArea & Increase & Increase & 12855672 & 11073891 \\
\hline LIF & SACT & Increase & Increase & 11009450 & 11073891,11387209 \\
\hline LIF & SERCA & Decrease & Decrease & 10752545 & \\
\hline NE & aMHC & Decrease & Decrease & 17592507 & \\
\hline NE & ANP & Increase & Increase & 1850419 & 20044473,17592507 \\
\hline NE & $\mathrm{bMHC}$ & Increase & Increase & 2560798 & $\begin{array}{l}2156896,2037558,20044473, \\
17592507\end{array}$ \\
\hline NE & BNP & Increase & Increase & 20044473 & 17592507 \\
\hline NE & CellArea & Increase & Increase & 6216022 & 20044473 \\
\hline NE & sACT & Increase & Increase & 2560798 & $\begin{array}{l}2821075,2537847,20044473 \\
17592507\end{array}$ \\
\hline NE & SERCA & Decrease & Decrease & 17592507 & \\
\hline NRG1 & aMHC & Decrease & No Data & & \\
\hline NRG1 & ANP & Increase & Increase & 9553078 & 10564160 \\
\hline NRG1 & $\mathrm{bMHC}$ & Increase & No Data & & \\
\hline NRG1 & BNP & Increase & Increase & 16698793 & \\
\hline NRG1 & CellArea & Increase & Increase & 9553078 & 16698793 \\
\hline NRG1 & SACT & Increase & Increase & 9553078 & \\
\hline NRG1 & SERCA & Decrease & No Data & & \\
\hline PE & aMHC & Decrease & Decrease & 15795322 & 9428623,17592507 \\
\hline $\mathrm{PE}$ & ANP & Increase & Increase & 15795322 & $11815436,10984495,11387209$, \\
\hline PE & $\mathrm{bMHC}$ & Increase & Increase & 15795322 & $10984495,10642269,17592507$ \\
\hline $\mathrm{PE}$ & BNP & Increase & Increase & 15795322 & $9428623,11387209,9798730$ \\
\hline PE & CellArea & Increase & Increase & 15795322 & 11815436,10984495 \\
\hline $\mathrm{PE}$ & SACT & Increase & Increase & 15795322 & $9428623,11387209,10642269$ \\
\hline $\mathrm{PE}$ & SERCA & Decrease & Decrease & 15795322 & 9428623,17592507 \\
\hline Stretch & aMHC & Decrease & Decrease & 9925362 & \\
\hline Stretch & ANP & Increase & Increase & 1534087 & 10784353,9925362 \\
\hline Stretch & bMHC & Increase & Increase & 1534087 & 9925362 \\
\hline Stretch & BNP & Increase & Increase & 10587524 & \\
\hline Stretch & CellArea & Increase & No Data & & \\
\hline Stretch & SACT & Increase & Increase & 1534087 & 1702436 \\
\hline Stretch & SERCA & Decrease & Decrease & 9925362 & \\
\hline TGFB & aMHC & Decrease & Decrease & 9428623 & 1688886,1712696 \\
\hline TGFB & ANP & Increase & Increase & 9428623 & $16125722,9396478,1712696$ \\
\hline TGFB & bMHC & Increase & Increase & 9428623 & $16125722,1688886,1712696$ \\
\hline TGFB & BNP & Increase & Increase & 16125722 & 9396478 \\
\hline TGFB & CellArea & Increase & Increase & 16125722 & 9396478 \\
\hline
\end{tabular}




\begin{tabular}{|l|l|l|l|l|l|l|} 
TGFB & & SACT & Increase & Increase & 9428623 & 1688886,1712696 \\
\hline TGFB & & SERCA & Decrease & Decrease & 9428623 & 1712696 \\
\hline TNFa & & aMHC & $\begin{array}{l}\text { No } \\
\text { Change }\end{array}$ & Decrease & 11827697 & \\
\hline TNFa & & ANP & Increase & Increase & 12409315 & 11851362 \\
\hline TNFa & & bMHC & Increase & No Data & & \\
\hline TNFa & & BNP & $\begin{array}{l}\text { No } \\
\text { Change }\end{array}$ & No Change & 12054852 & \\
\hline TNFa & & CellArea & Increase & Increase & 12409315 & 9727550,11851362 \\
\hline TNFa & & SACT & $\begin{array}{l}\text { No } \\
\text { Change }\end{array}$ & No Data & & \\
\hline TNFa & & SERCA & $\begin{array}{l}\text { No } \\
\text { Change }\end{array}$ & No Data & & \\
\hline
\end{tabular}




\section{Appendix E}

CITED4 and myocyte elongation model 


\section{Supplementary Methods:}

Equations for CITED4 and Elongation Model:
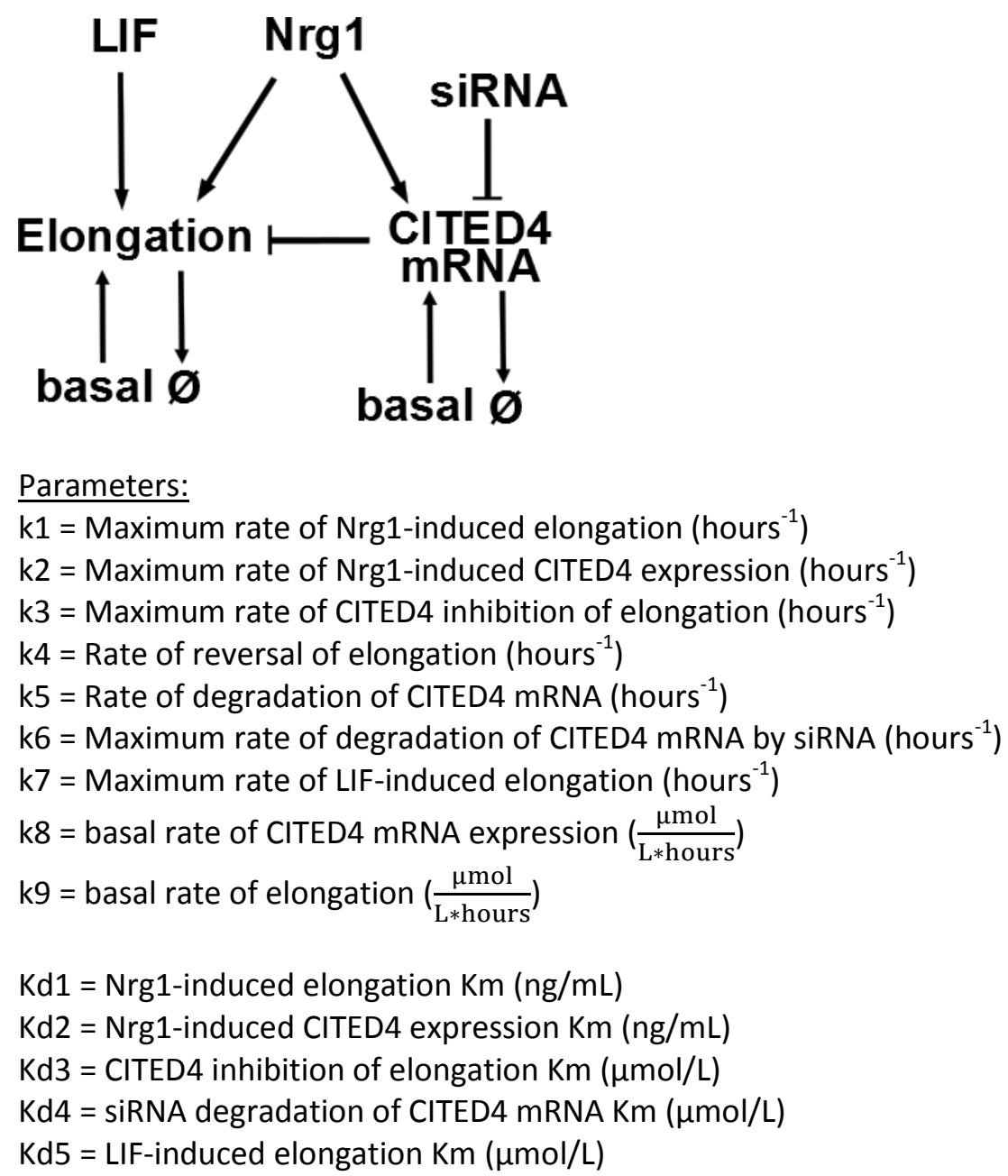

Parameters:

$\mathrm{k} 1$ = Maximum rate of Nrg1-induced elongation (hours ${ }^{-1}$ )

k2 = Maximum rate of Nrg1-induced CITED4 expression (hours ${ }^{-1}$ )

k3 = Maximum rate of CITED4 inhibition of elongation (hours ${ }^{-1}$ )

k4 = Rate of reversal of elongation (hours ${ }^{-1}$ )

$\mathrm{k} 5$ = Rate of degradation of CITED4 mRNA (hours ${ }^{-1}$ )

k6 = Maximum rate of degradation of CITED4 mRNA by siRNA (hours ${ }^{-1}$ )

k7 = Maximum rate of LIF-induced elongation (hours ${ }^{-1}$ )

$\mathrm{k} 8$ = basal rate of CITED4 mRNA expression $\left(\frac{\mu \mathrm{mol}}{\mathrm{L} * \text { hours }}\right)$

$\mathrm{k} 9=$ basal rate of elongation $\left(\frac{\mu \mathrm{mol}}{\mathrm{L} * \text { hours }}\right)$

$\mathrm{Kd} 1=\mathrm{Nrg} 1$-induced elongation $\mathrm{Km}(\mathrm{ng} / \mathrm{mL})$

$\mathrm{Kd} 2=\mathrm{Nrg} 1$-induced CITED4 expression $\mathrm{Km}(\mathrm{ng} / \mathrm{mL})$

$\mathrm{Kd} 3=\mathrm{CITED} 4$ inhibition of elongation $\mathrm{Km}(\mu \mathrm{mol} / \mathrm{L})$

$\mathrm{Kd} 4=$ siRNA degradation of CITED4 mRNA Km $(\mu \mathrm{mol} / \mathrm{L})$

$\mathrm{Kd} 5=\mathrm{LIF}$-induced elongation $\mathrm{Km}(\mu \mathrm{mol} / \mathrm{L})$

Parameter Fitting Results:

The following parameters were estimated by nonlinear least-squares fitting to the control siRNA experimental data in Figure 7A-B using the "Isqnonlin" function in Matlab:

$$
\begin{aligned}
& \text { k1 }=0.0219 \text { hours }^{-1} \\
& \text { k2 }=0.0329 \text { hours }^{-1} \\
& \text { k3 }=0.0219 \text { hours }^{-1} \\
& \text { k4 }=0.0083 \text { hours }^{-1} \\
& \text { k5 }=0.01 \text { hours }^{-1} \\
& \text { k6 }=0.05 \text { hours }^{-1} \\
& \text { k7 }=0.025 \text { hours }^{-1} \\
& \text { k8 }=0.01 \frac{\mu \text { mol }}{\mathrm{L} * \text { hours }} \\
& \text { k9 }=0.0083 \frac{\mu \text { mol }}{\mathrm{L} * \text { hours }}
\end{aligned}
$$


$\mathrm{Kd} 1=1 \mathrm{ng} / \mathrm{mL}$

$\mathrm{Kd} 2=1 \mathrm{ng} / \mathrm{mL}$

$\mathrm{Kd} 3=1.1707 \mu \mathrm{mol} / \mathrm{L}$

$\mathrm{Kd} 4=1 \mu \mathrm{mol} / \mathrm{L}$

$\mathrm{Kd} 5=1.1545 \mu \mathrm{mol} / \mathrm{L}$

Differential Equations:

$\frac{d \text { CITED } 4}{d t}=k 8+\frac{k 2 * N r g 1}{K d 2+N r g 1}-k 5 *$ CITED $4-k 6 * \operatorname{siRNA} *$ CITED 4

$\frac{d \text { Elongation }}{d t}=k 9+\frac{k 1 * N r g 1}{K d 1+N r g 1}+\frac{k 7 * L I F}{K d 5+L I F}-\frac{k 3 * \text { CITED } 4}{K d 3+\text { CITED } 4}-k 4 *$ Elongation

If, fold change in Elongation $<1, k 3=0$

\section{Equations for Elongation and CITED4 model variations:}

Model without CITED4 inhibition of elongation:

Parameter Fitting Results:

The following parameters were estimated by nonlinear least-squares fitting to the control siRNA experimental data in Figure 7A-B using the "Isqnonlin" function in Matlab:

$\mathrm{k} 1=0.0135$ hours $^{-1}$

$\mathrm{k} 2=0.0329$ hours $^{-1}$

$\mathrm{k} 3=\mathrm{N} / \mathrm{A}$

$\mathrm{k} 4=0.0427$ hours $^{-1}$

$\mathrm{k} 5=0.01$ hours $^{-1}$

k6 $=0.065$ hours $^{-1}$

$\mathrm{k} 7=0.0059$ hours $^{-1}$

$\mathrm{k} 8=0.01 \frac{\mu \mathrm{mol}}{\mathrm{L} * \text { hours }}$

k9 $=0.0135 \frac{\mu \text { mol }}{\mathrm{L} * \text { hours }}$

$\mathrm{Kd} 1=1 \mathrm{ng} / \mathrm{mL}$

$\mathrm{Kd} 2=1 \mathrm{ng} / \mathrm{mL}$

$\mathrm{Kd} 3=\mathrm{N} / \mathrm{A}$

$\mathrm{Kd} 4=1 \mu \mathrm{mol} / \mathrm{L}$

$\mathrm{Kd} 5=1.1545 \mu \mathrm{mol} / \mathrm{L}$ 
Differential Equations:

$\frac{d C I T E D 4}{d t}=k 8+\frac{k 2 * N r g 1}{K d 2+N r g 1}-k 5 * C I T E D 4-k 6 * s i R N A * C I T E D 4$

$\frac{d \text { Elongation }}{d t}=k 9+\frac{k 1 * N r g 1}{K d 1+N r g 1}+\frac{k 7 * L I F}{K d 5+L I F}-k 4 *$ Elongation

Model without minimum elongation value for CITED4 inhibition:

Parameter Fitting Results:

The following parameters were estimated by nonlinear least-squares fitting to the control siRNA experimental data in Figure 7A-B using the "Isqnonlin" function in Matlab:

$\mathrm{k} 1=0.0155$ hours $^{-1}$

$\mathrm{k} 2=0.0329$ hours $^{-1}$

$\mathrm{k} 3=0.0117$ hours $^{-1}$

$\mathrm{k} 4=0.0132$ hours $^{-1}$

$\mathrm{k} 5=0.01$ hours $^{-1}$

$k 6=0.065$ hours $^{-1}$

k7 $=0.015$ hours $^{-1}$

k8 $=0.01 \frac{\mu \mathrm{mol}}{\mathrm{L} * \text { hours }}$

$\mathrm{k} 9=0.0134 \frac{\mu \mathrm{mol}}{\mathrm{L} * \text { hours }}$

$\mathrm{Kd} 1=1 \mathrm{ng} / \mathrm{mL}$

$\mathrm{Kd} 2=1 \mathrm{ng} / \mathrm{mL}$

$\mathrm{Kd} 3=1 \mu \mathrm{mol} / \mathrm{L}$

$\mathrm{Kd} 4=1 \mu \mathrm{mol} / \mathrm{L}$

$\mathrm{Kd} 5=1 \mu \mathrm{mol} / \mathrm{L}$

Differential Equations:

$\frac{d C I T E D 4}{d t}=k 8+\frac{k 2 * N r g 1}{K d 2+N r g 1}-k 5 * C I T E D 4-k 6 * \operatorname{siRNA} *$ CITED 4

$\frac{\text { dElongation }}{d t}=k 9+\frac{k 1 * N r g 1}{K d 1+N r g 1}+\frac{k 7 * L I F}{K d 5+L I F}-\frac{k 3 * \text { CITED } 4}{K d 3+C I T E D 4}-k 4 *$ Elongation 


\section{References}

1. Braunwald, E. Research advances in heart failure: a compendium. Circulation research 113, 633-45 (2013).

2. Heidenreich, P. A. et al. Forecasting the impact of heart failure in the United States: a policy statement from the American Heart Association. Circulation. Heart failure 6, 606-19 (2013).

3. Mudd, J.O. \& Kass, D.A. Tackling heart failure in the twenty-first century. Nature $451,919-28$ (2008).

4. Sanganalmath, S.K. \& Bolli, R. Cell therapy for heart failure: a comprehensive overview of experimental and clinical studies, current challenges, and future directions. Circulation research 113, 810-34 (2013).

5. Taylor, C.J., Roalfe, A.K., lles, R. \& Hobbs, F.D.R. Ten-year prognosis of heart failure in the community: follow-up data from the Echocardiographic Heart of England Screening (ECHOES) study. European journal of heart failure 14, 176-84 (2012).

6. Goldberg, R.J., Ciampa, J., Lessard, D., Meyer, T.E. \& Spencer, F.A. Long-term Survival After Heart Failure. Arch Intern Med 167, 490-496 (2007).

7. Jessup, M. \& Brozena, S. Heart failure. The New England journal of medicine $\mathbf{3 4 8 , 2 0 0 7 - 1 8 ~ ( 2 0 0 3 ) . ~}$

8. Bui, A.L., Horwich, T.B. \& Fonarow, G.C. Epidemiology and risk profile of heart failure. Nature reviews. Cardiology 8, 30-41 (2011).

9. Stewart, S., Maclntyre, K., Hole, D.J., Capewell, S. \& McMurray, J.J. More "malignant" than cancer? Five-year survival following a first admission for heart failure. European journal of heart failure 3, 315-22 (2001).

10. Levy, D., Garrison, R., Savage, D., Kannel, W. \& Castelli, W. Prognostic implications of echocardiographically determined left ventricular mass in the Framingham Heart Study. NEJM 322, 1561-1566 (1990).

11. Okin, P.M. et al. Regression of electrocardiographic left ventricular hypertrophy during antihypertensive treatment and the prediction of major cardiovascular events. JAMA : the journal of the American Medical Association 292, 2343-9 (2004).

12. Hill, J.A. \& Olson, E.N. Cardiac plasticity. The New England journal of medicine 358, 1370-80 (2008).

13. Heineke, J. \& Molkentin, J.D. Regulation of cardiac hypertrophy by intracellular signalling pathways. Nature reviews. Molecular cell biology 7, 589-600 (2006).

14. Koitabashi, N. \& Kass, D. a Reverse remodeling in heart failure-mechanisms and therapeutic opportunities. Nature reviews. Cardiology 1-11 (2011).doi:10.1038/nrcardio.2011.172 
15. Massie, B.M. Novel targets for the treatment of heart failure: Perspectives from a heart failure clinician and trialist. Journal of molecular and cellular cardiology 51, 438-40 (2011).

16. Plump, A. \& Madwed, J. Heart failure drug discovery: Scarred or ready for remodeling? Journal of molecular and cellular cardiology 51, 444-8 (2011).

17. Bernardo, B.C., Weeks, K.L., Pretorius, L. \& McMullen, J.R. Molecular distinction between physiological and pathological cardiac hypertrophy: Experimental findings and therapeutic strategies. Pharmacology \& therapeutics 128, 191-227 (2010).

18. Molkentin, J.D. The transcription factor C/EBPbeta serves as a master regulator of physiologic cardiac hypertrophy. Circulation research 108, 277-8 (2011).

19. Strøm, C.C. et al. Expression profiling reveals differences in metabolic gene expression between exercise-induced cardiac effects and maladaptive cardiac hypertrophy. The FEBS journal 272, 2684-95 (2005).

20. Scheinowitz, M. et al. Short- and long-term swimming exercise training increases myocardial insulin-like growth factor-I gene expression. Growth hormone \& IGF research 13, 19-25 (2003).

21. Neri Serneri, G.G. et al. Increased Cardiac Sympathetic Activity and Insulin-Like Growth Factor-I Formation Are Associated With Physiological Hypertrophy in Athletes. Circulation Research 89, 977-982 (2001).

22. Yayama, K. et al. Up-Regulation of Angiotensin II Type 2 Receptor in Rat Thoracic Aorta by Pressure-Overload. Pharmacology 308, 736-743 (2004).

23. Rapacciuolo, a et al. Important role of endogenous norepinephrine and epinephrine in the development of in vivo pressure-overload cardiac hypertrophy. Journal of the American College of Cardiology 38, 876-82 (2001).

24. Arai, M. et al. Endothelin-1 and its binding sites are upregulated in pressure overload cardiac hypertrophy Endothelin-1 and its binding sites are upregulated in pressure overload cardiac hypertrophy. In Situ (2012).

25. Mcmullen, J.R. et al. Phosphoinositide 3-kinase(p110 $\alpha$ ) plays a critical role for the induction of physiological, but not pathological, cardiac hypertrophy. PNAS 100, 12355-12360 (2003).

26. Devereux, R.B. Therapeutic options in minimizing left ventricular hypertrophy. American heart journal 139, S9-14 (2000).

27. Geenen, D.L., Malhotra, A., Buttrick, P.M. \& Geenen, D.L. Angiotensin receptor 1 blockade does not prevent physiological cardiac hypertrophy in the adult rat. J Appl Physio/ 81, 816-821 (1996).

28. Boström, P. et al. C/EBP $\beta$ controls exercise-induced cardiac growth and protects against pathological cardiac remodeling. Cell 143, 1072-83 (2010). 
29. Grossman, W., Jones, D. \& McLaurin, L.P. Wall stress and patterns of hypertrophy in the human left ventricle. The Journal of clinical investigation 56, 56-64 (1975).

30. Kehat, l. et al. Extracellular signal-regulated kinases 1 and 2 regulate the balance between eccentric and concentric cardiac growth. Circulation research 108, 176-83 (2011).

31. Berenji, K., Drazner, M.H., Rothermel, B. a \& Hill, J. a Does load-induced ventricular hypertrophy progress to systolic heart failure? American journal of physiology. Heart and circulatory physiology 289, H8-H16 (2005).

32. Dorn, G.W., Robbins, J. \& Sugden, P.H. Phenotyping hypertrophy: eschew obfuscation. Circulation research 92, 1171-5 (2003).

33. McCulloch, A.D. \& Paternostro, G. Cardiac systems biology. Annals of the New York Academy of Sciences 1047, 283-95 (2005).

34. Lusis, A.J. \& Weiss, J.N. Cardiovascular networks: systems-based approaches to cardiovascular disease. Circulation 121, 157-70 (2010).

35. Evans, J.G. \& Matsudaira, P. Linking microscopy and high content screening in large-scale biomedical research. Methods Mol Biol 356, 33-38 (2007).

36. Chan, S.Y. \& Loscalzo, J. The emerging paradigm of network medicine in the study of human disease. Circulation research 111, 359-74 (2012).

37. Bass, G.T. et al. Automated image analysis identifies signaling pathways regulating distinct signatures of cardiac myocyte hypertrophy. Journal of molecular and cellular cardiology (2011).doi:10.1016/j.yjmcc.2011.11.009

38. Jentzsch, C. et al. A phenotypic screen to identify hypertrophy-modulating microRNAs in primary cardiomyocytes. Journal of molecular and cellular cardiology (2011).doi:10.1016/j.yjmcc.2011.07.010

39. Ryall, K.A. \& Saucerman, J.J. Automated imaging reveals concentration dependent delay in reversibility of cardiac myocyte hypertrophy. J Mol Cell Cardiol (2012).doi:10.1016/j.yjmcc.2012.04.016

40. Takimoto, E. et al. Chronic inhibition of cyclic GMP phosphodiesterase 5A prevents and reverses cardiac hypertrophy. Nature medicine 11, 214-22 (2005).

41. Xu, D. et al. Prevention and reversal of cardiac hypertrophy by soluble epoxide hydrolase inhibitors. Proceedings of the National Academy of Sciences of the United States of America 103, 18733-8 (2006).

42. Moens, A.L. et al. Reversal of cardiac hypertrophy and fibrosis from pressure overload by tetrahydrobiopterin: efficacy of recoupling nitric oxide synthase as a therapeutic strategy. Circulation 117, 2626-36 (2008). 
43. Zafeiridis, A., Jeevanandam, V., Houser, S.R. \& Margulies, K.B. Regression of cellular hypertrophy after left ventricular assist device support. Circulation 98, 656-62 (1998).

44. Kuhn, M. et al. Left ventricular assist device support reverses altered cardiac expression and function of natriuretic peptides and receptors in end-stage heart failure. Cardiovascular research 64, 308-14 (2004).

45. Birks, E.J. et al. Reversal of severe heart failure with a continuous-flow left ventricular assist device and pharmacological therapy: a prospective study. Circulation 123, 381-90 (2011).

46. Jentzsch, C. et al. A phenotypic screen to identify hypertrophy-modulating microRNAs in primary cardiomyocytes. Journal of molecular and cellular cardiology (2011).doi:10.1016/j.yjmcc.2011.07.010

47. Prasad, K.-M.R., Xu, Y., Yang, Z., Acton, S.T. \& French, B.A. Robust cardiomyocyte-specific gene expression following systemic injection of $A A V$ : in vivo gene delivery follows a Poisson distribution. Gene therapy 43-52 (2010).doi:10.1038/gt.2010.105

48. Otsu, N. A Threshold Selection Method from Gray-Level Histograms. IEEE Transactions on Systems, Man, and Cybernetics 9, 62-66 (1979).

49. Vincent, L. \& Soille, P. Watersheds in Digital Spaces: An Efficient Algorithm Based on Immersion Simulations. IEEE transactions on pattern analysis and machine intelligence 13, 583-97 (1991).

50. Carpenter, A.E. et al. CellProfiler: image analysis software for identifying and quantifying cell phenotypes. Genome biology 7, R100 (2006).

51. Xia, Y., Rajapurohitam, V., Cook, M.A. \& Karmazyn, M. Inhibition of phenylephrine induced hypertrophy in rat neonatal cardiomyocytes by the mitochondrial K ATP channel opener diazoxide. Journal of Molecular and Cellular Cardiology 37, 1063-1067 (2004).

52. Barron, A.J., Finn, S.G. \& Fuller, S.J. Chronic activation of extracellular-signal-regulated protein kinases by phenylephrine is required to elicit a hypertrophic response in cardiac myoctes. Biochem. J. 371, 71-79 (2003).

53. Pediani, J.D. et al. B-arrestin-dependent spontaneous $\alpha 1$ a-adrenoceptor endocytosis causes intracellular transportation of $\alpha$-blockers via recycling compartments. Molecular Pharmacology 992-1004 (2005).doi:10.1124/mol.104.008417.uptake

54. Wright, C.D. et al. Nuclear alpha1-adrenergic receptors signal activated ERK localization to caveolae in adult cardiac myocytes. Circulation research 103, 992-1000 (2008).

55. Levin, M.C., Marullo, S., Muntaner, O., Andersson, B. \& Magnusson, Y. The myocardiumprotective Gly-49 variant of the beta 1-adrenergic receptor exhibits constitutive activity and increased desensitization and down-regulation. The Journal of biological chemistry 277, 30429-35 (2002). 
56. Staehelin, M., Simons, P., Jaeggi, K. \& Wigger, N. CGP 12177: A hydrophilic B-adrenergic receptor radioligand reveals high affinity binding of agonists to intact cells. The Journal of biological chemistry 258, 3496-3502 (1983).

57. Brahmadevara, N., Shaw, A.M. \& MacDonald, A. ALpha1-adrenoceptor antagonist properties of CGP 12177A and other beta-adrenoceptor ligands: evidence against beta(3)- or atypical betaadrenoceptors in rat aorta. British journal of pharmacology 142, 781-7 (2004).

58. Brahmadevara, N., Shaw, A.M. \& MacDonald, A. Evidence against beta 3-adrenoceptors or low affinity state of beta 1-adrenoceptors mediating relaxation in rat isolated aorta. British journal of pharmacology 138, 99-106 (2003).

59. Sadoshima, J. \& Izumo, S. The cellular and molecular response of cardiac myocytes to mechanical stress. Annual review of physiology 59, 551-71 (1997).

60. Nishimura, J., Kanaide, H. \& Nakamura, M. Binding of [3H]Prazosin of Calcium Antagonists. The Journal of Pharmacology and Experimental Therapeutics 236, 789-93 (1986).

61. Daly, C.J., Milligan, C.M., Milligan, G., Mackenzie, J.F. \& Mcgrath, J.C. Cellular localization and pharmacological characterization of functioning alpha-1 adrenoceptors by fluorescent ligand binding and image analysis reveals identical binding properties of clustered and diffuse populations of receptors. The Journal of pharmacology and experimental therapeutics 286, 98490 (1998).

62. Mackenzie, J.F., Daly, C.J., Pediani, J.D. \& McGrath, J.C. Quantitative imaging in live human cells reveals intracellular alpha(1)-adrenoceptor ligand-binding sites. The Journal of pharmacology and experimental therapeutics 294, 434-43 (2000).

63. Tadevosyan, A. et al. Nuclear-delimited angiotensin receptor-mediated signaling regulates cardiomyocyte gene expression. The Journal of biological chemistry 285, 22338 -22349 (2010).

64. Boivin, B., Chevalier, D., Villeneuve, L.R., Rousseau, E. \& Allen, B.G. Functional endothelin receptors are present on nuclei in cardiac ventricular myocytes. The Journal of biological chemistry 278, 29153-63 (2003).

65. Boivin, B. et al. Functional beta-adrenergic receptor signalling on nuclear membranes in adult rat and mouse ventricular cardiomyocytes. Cardiovascular research 71, 69-78 (2006).

66. Berry, J.M. et al. Reversibility of Adverse, Calcineurin-Dependent Cardiac Remodeling. Circulation research (2011).doi:10.1161/CIRCRESAHA.110.228452

67. Moretti, A. et al. Patient-specific induced pluripotent stem-cell models for long-QT syndrome. The New England journal of medicine 363, 1397-409 (2010).

68. Saucerman, J.J., Brunton, L.L., Michailova, A.P. \& McCulloch, A.D. Modeling beta-adrenergic control of cardiac myocyte contractility in silico. The Journal of biological chemistry 278, 479978003 (2003). 
69. Saucerman, J.J. et al. Computational Models of Whole Cells and Heterogeneous Tissue. Circulation Research 1216-1224 (2004).doi:10.1161/01.RES.0000150055.06226.4e

70. Shin, S.-young et al. Switching feedback mechanisms realize the dual role of MCIP in the regulation of calcineurin activity. FEBS Letters 580, 5965-5973 (2006).

71. Cooling, M., Hunter, P. \& Crampin, E.J. Modeling Hypertrophic IP3 Transients in the Cardiac Myocyte. Biophysical Journal 93, 3421-3433 (2007).

72. Ibarra, R.U., Edwards, J.S. \& Palsson, B.O. Escherichia coli K-12 undergoes adaptive evolution to achieve in silico predicted optimal growth. Nature 420, 20-23 (2002).

73. Chavali, A.K., Whittemore, J.D., Eddy, J.A., Williams, K.T. \& Papin, J.A. Systems analysis of metabolism in the pathogenic trypanosomatid Leishmania major. Molecular Systems Biology (2008).doi:10.1038/msb.2008.15

74. Bonneau, R. et al. Resource A Predictive Model for Transcriptional Control of Physiology in a Free Living Cell. Cell 1354-1365 (2007).doi:10.1016/j.cell.2007.10.053

75. Kraeutler, M.J., Soltis, A.R. \& Saucerman, J.J. Modeling cardiac $\beta$-adrenergic signaling with normalized-Hill differential equations: comparison with a biochemical model. BMC systems biology 4, 157 (2010).

76. Shannon, P. et al. Cytoscape: a software environment for integrated models of biomolecular interaction networks. Genome research 13, 2498-504 (2003).

77. Assenov, Y., Ramírez, F., Schelhorn, S.-E., Lengauer, T. \& Albrecht, M. Computing topological parameters of biological networks. Bioinformatics (Oxford, England) 24, 282-4 (2008).

78. Ferro, A. et al. Sequence similarity is more relevant than species specificity in probabilistic backtranslation. BMC bioinformatics 8, 58 (2007).

79. Alon, U. Network motifs: theory and experimental approaches. Nature reviews. Genetics 8, 450-61 (2007).

80. Omura, T. et al. Dominant Negative Mutant of c-Jun Inhibits Cardiomyocyte Hypertrophy Induced by Endothelin 1 and Phenylephrine. Hypertension 39, 81-86 (2002).

81. H. Jeong, S. P. Mason, A.-L.B.\& Z.N.O. Lethality and centrality in protein networks. Nature 411, 4142 (2001).

82. Schaub, M.C., Hefti, M.A., Harder, B.A. \& Eppenberger, H.M. Various hypertrophic stimuli induce distinct phenotypes in cardiomyocytes. J Mol Med 75, 901-920 (1997).

83. Jeong, H. Error and attack tolerance of complex networks'. Nature 406, (2000). 
84. Bain, J. et al. The selectivity of protein kinase inhibitors: a further update. The Biochemical journal 408, 297-315 (2007).

85. Hung, W. \& Chaung, L. The farnesyltransferase inhibitor, FPT inhibitor III upregulates Bax and Bclxs expression and induces apoptosis in human ovarian cancer cells. In. J. Oncol. 12, 137-77 (1998).

86. Morgan, M. a. Cell-cycle-dependent activation of mitogen-activated protein kinase kinase (MEK$1 / 2$ ) in myeloid leukemia cell lines and induction of growth inhibition and apoptosis by inhibitors of RAS signaling. Blood 97, 1823-1834 (2001).

87. Kalir, S., Mangan, S. \& Alon, U. A coherent feed-forward loop with a SUM input function prolongs flagella expression in Escherichia coli. Molecular Systems Biology 1-6 (2005).doi:10.1038/msb4100010

88. Mangan, S. \& Alon, U. Structure and function of the feed-forward loop network motif. October 100, (2003).

89. Hartwell, L.H., Hopfield, J.J., Leibler, S. \& Murray, A.W. From molecular to modular cell biology. Nature 402, 47-52 (1999).

90. Antos, C.L. et al. Activated glycogen synthase-3 "`. suppresses cardiac hypertrophy in vivo. (2001).

91. Sanbe, A. et al. Reengineering Inducible Cardiac-Specific Transgenesis With an Attenuated Myosin Heavy Chain Promoter. Circulation Research 609-616 (2003).doi:10.1161/01.RES.0000065442.64694.9F

92. Yue, T.L. et al. Extracellular signal-regulated kinase plays an essential role in hypertrophic agonists, endothelin-1 and phenylephrine-induced cardiomyocyte hypertrophy. The Journal of biological chemistry 275, 37895-901 (2000).

93. Zechner, D., Thuerauf, D.J., Hanford, D.S., McDonough, P.M. \& Glembotski, C.C. A role for the p38 mitogen-activated protein kinase pathway in myocardial cell growth, sarcomeric organization, and cardiac-specific gene expression. The Journal of cell biology 139, 115-27 (1997).

94. Fuller, S.J., Gillespie-Brown, J. \& Sugden, P.H. Oncogenic src, raf, and ras stimulate a hypertrophic pattern of gene expression and increase cell size in neonatal rat ventricular myocytes. The Journal of biological chemistry 273, 18146-52 (1998).

95. Wang, Y. et al. Cardiac hypertrophy induced by mitogen-activated protein kinase kinase 7, a specific activator for c-Jun $\mathrm{NH2}$-terminal kinase in ventricular muscle cells. The Journal of biological chemistry 273, 5423-6 (1998).

96. Ueyama, T. et al. Requirement of activation of the extracellular signal-regulated kinase cascade in myocardial cell hypertrophy. Journal of molecular and cellular cardiology 32, 947-60 (2000). 
97. Rose, B.A., Force, T. \& Wang, Y. Mitogen-Activated Protein Kinase Signaling in the Heart : Angels Versus Demons in a Heart-Breaking Tale. Phsiol Rev 1507-1546 (2010).doi:10.1152/physrev.00054.2009.

98. Levy, D., Garrison, R., Savage, D., Kannel, W. \& Castelli, W. Prognostic implications of echocardiographically determined left ventricular mass in the Framingham Heart Study. NEJM 322, 1561-1566 (1990).

99. Okin, P.M. et al. Regression of electrocardiographic left ventricular hypertrophy during antihypertensive treatment and the prediction of major cardiovascular events. JAMA : the journal of the American Medical Association 292, 2343-9 (2004).

100. Haider, A. W., Larson, M.G., Benjamin, E.J. \& Levy, D. Increased left ventricular mass and hypertrophy are associated with increased risk for sudden death. Journal of the American College of Cardiology 32, 1454-9 (1998).

101. Berlo, J.H.V., Maillet, M. \& Molkentin, J.D. Review series Signaling effectors underlying pathologic growth and remodeling of the heart. Conflict 123, 37-45 (2013).

102. Maillet, M., van Berlo, J.H. \& Molkentin, J.D. Molecular basis of physiological heart growth: fundamental concepts and new players. Nature reviews. Molecular cell biology 14, 38-48 (2013).

103. Grossman, W., Jones, D. \& McLaurin, L.P. Wall stress and patterns of hypertrophy in the human left ventricle. J Clin Invest 56, 56-64 (1975).

104. Burchfield, J.S., Xie, M. \& Hill, J. a Pathological ventricular remodeling: mechanisms: part 1 of 2. Circulation 128, 388-400 (2013).

105. Kehat, I. et al. Extracellular signal-regulated kinases 1 and 2 regulate the balance between eccentric and concentric cardiac growth. Circulation research 108, 176-83 (2011).

106. Kehat, I. \& Molkentin, J.D. Molecular pathways underlying cardiac remodeling during pathophysiological stimulation. Circulation 122, 2727-35 (2010).

107. Ryall, K. A. et al. Network reconstruction and systems analysis of cardiac myocyte hypertrophy signaling. The Journal of biological chemistry 287, 42259-68 (2012).

108. Schmittgen, T.D. \& Livak, K.J. Analyzing real-time PCR data by the comparative CT method. Nature Protocols 3, 1101-1108 (2008).

109. Sachs, K., Perez, O., Pe'er, D., Lauffenburger, D. a \& Nolan, G.P. Causal protein-signaling networks derived from multiparameter single-cell data. Science (New York, N.Y.) 308, 523-9 (2005).

110. Izu, L.T. \& Chen-Izu, Y. Une cellule type? Journal of molecular and cellular cardiology 52, 921-2 (2012). 
111. Whelan, R.S. et al. Bax regulates primary necrosis through mitochondrial dynamics. Proceedings of the National Academy of Sciences of the United States of America 109, 6566-71 (2012).

112. Crow, M.T., Mani, K., Nam, Y.-J. \& Kitsis, R.N. The mitochondrial death pathway and cardiac myocyte apoptosis. Circulation research 95, 957-70 (2004).

113. Nadal-Ginard, B. Myocyte Death, Growth, and Regeneration in Cardiac Hypertrophy and Failure. Circulation Research 92, 139-150 (2003).

114. Shiojima, I. et al. Disruption of coordinated cardiac hypertrophy and angiogenesis contributes to the transition to heart failure. $J C l$ 115, (2005).

115. Periasamy, M. \& Huke, S. SERCA pump level is a critical determinant of $\mathrm{Ca}(2+)$ homeostasis and cardiac contractility. Journal of molecular and cellular cardiology 33, 1053-63 (2001).

116. Kuwahara, K. et al. NRSF regulates the fetal cardiac gene program and maintains normal cardiac structure and function. The EMBO journal 22, 6310-21 (2003).

117. Freund, C. et al. Requirement of nuclear factor-kappaB in angiotensin II- and isoproterenolinduced cardiac hypertrophy in vivo. Circulation 111, 2319-25 (2005).

118. Sun, M. et al. Tumor necrosis factor-alpha mediates cardiac remodeling and ventricular dysfunction after pressure overload state. Circulation 115, 1398-407 (2007).

119. Nian, M., Lee, P., Khaper, N. \& Liu, P. Inflammatory cytokines and postmyocardial infarction remodeling. Circulation research 94, 1543-53 (2004).

120. Kemp, T.J., Aggeli, I.-K., Sugden, P.H. \& Clerk, A. Phenylephrine and endothelin-1 upregulate connective tissue growth factor in neonatal rat cardiac myocytes. Journal of molecular and cellular cardiology 37, 603-6 (2004).

121. Tirziu, D., Giordano, F.J. \& Simons, M. Cell communications in the heart. Circulation 122, 928-37 (2010).

122. Baines, C.P. \& Molkentin, J.D. Adenine nucleotide translocase-1 induces cardiomyocyte death through upregulation of the pro-apoptotic protein Bax. Journal of Molecular and Cellular Cardiology 46, 969-977 (2009).

123. Del Re, D.P., Miyamoto, S. \& Brown, J.H. RhoA/Rho kinase up-regulate Bax to activate a mitochondrial death pathway and induce cardiomyocyte apoptosis. The Journal of biological chemistry 282, 8069-78 (2007).

124. Smith, R.M. et al. Genetic depletion of cardiac myocyte STAT-3 abolishes classical preconditioning. Cardiovascular research 63, 611-6 (2004).

125. Kubli, D. A., Ycaza, J.E. \& Gustafsson, A.B. Bnip3 mediates mitochondrial dysfunction and cell death through Bax and Bak. The Biochemical journal 405, 407-15 (2007). 
126. Cook, S. A., Clerk, A. \& Sugden, P.H. Are transgenic mice the "alkahest" to understanding myocardial hypertrophy and failure? Journal of molecular and cellular cardiology 46, 118-29 (2009).

127. Natarajan, M., Lin, K.-M., Hsueh, R.C., Sternweis, P.C. \& Ranganathan, R. A global analysis of crosstalk in a mammalian cellular signalling network. Nature cell biology 8, 571-80 (2006).

128. Jaba, I.M. et al. NO triggers RGS4 degradation to coordinate angiogenesis and cardiomyocyte growth. $J C l$ 123, 1718-31 (2013).

129. Kwak, H. B., Song, W. \& Lawler, J.M. Exercise training attenuates age-induced elevation in $\mathrm{Bax} / \mathrm{BCl}-2$ ratio, apoptosis, and remodeling in the rat heart. FASEB journal : official publication of the Federation of American Societies for Experimental Biology 20, 791-3 (2006).

130. Misao, J. et al. Expression of bcl-2 Protein, an Inhibitor of Apoptosis, and Bax, an Accelerator of Apoptosis, in Ventricular Myocytes of Human Hearts With Myocardial Infarction. Circulation 94, 1506-12 (1996).

131. Dorn, G.W. Apoptotic and non-apoptotic programmed cardiomyocyte death in ventricular remodelling. Cardiovascular research 81, 465-73 (2009).

132. Hayakawa, Y. et al. Inhibition of cardiac myocyte apoptosis improves cardiac function and abolishes mortality in the peripartum cardiomyopathy of Galpha(q) transgenic mice. Circulation 108, 3036-41 (2003).

133. Creemers, E.E. \& Pinto, Y.M. Molecular mechanisms that control interstitial fibrosis in the pressure-overloaded heart. Cardiovascular research 89, 265-72 (2011).

134. Assayag, P. et al. Compensated cardiac hypertrophy: arrhythmogenicity and the new myocardial phenotype. I. Fibrosis. Cardiovascular research 34, 439-44 (1997).

135. Rupérez, M. et al. Connective tissue growth factor is a mediator of angiotensin II-induced fibrosis. Circulation 108, 1499-505 (2003).

136. Hishikawa, K. et al. Connective Tissue Growth Factor Induces Apoptosis in Human Breast Cancer Cell Line MCF-7. Journal of Biological Chemistry 274, 37461-37466 (1999).

137. Hishikawa, K., Nakaki, T. \& Fujii, T. Connective tissue growth factor induces apoptosis via caspase 3 in cultured human aortic smooth muscle cells. European journal of pharmacology 392, 19-22 (2000).

138. Hishikawa, K. et al. Overexpression of Connective Tissue Growth Factor Gene Induces Apoptosis in Human Aortic Smooth Muscle Cells. Circulation 100, 2108-2112 (1999).

139. Dean, R.G. et al. Connective tissue growth factor and cardiac fibrosis after myocardial infarction. The journal of histochemistry and cytochemistry : official journal of the Histochemistry Society 53, 1245-56 (2005). 
140. Mangan, S., Itzkovitz, S., Zaslaver, a \& Alon, U. The incoherent feed-forward loop accelerates the response-time of the gal system of Escherichia coli. Journal of molecular biology 356, 1073-81 (2006).

141. Kuramochi, Y., Guo, X. \& Sawyer, D.B. Neuregulin activates erbB2-dependent src / FAK signaling and cytoskeletal remodeling in isolated adult rat cardiac myocytes. J Mol Cell Cardiol 41, 228-235 (2006).

142. García-Rivello, H. et al. Dilated cardiomyopathy in Erb-b4-deficient ventricular muscle. American journal of physiology. Heart and circulatory physiology 289, H1153-60 (2005).

143. Sawyer, D.B. \& Caggiano, A. Neuregulin-1 $\beta$ for the treatment of systolic heart failure. Journal of molecular and cellular cardiology 51, 501-5 (2011).

144. Velagaleti, R.S. et al. Left Ventricular Hypertrophy Patterns and Incidence of Heart Failure With Preserved Versus Reduced Ejection Fraction. The American journal of cardiology 1-6 (2013).doi:10.1016/j.amjcard.2013.09.028

145. Nicol, R.L. et al. Activated MEK5 induces serial assembly of sarcomeres and eccentric cardiac hypertrophy. The EMBO journal 20, 2757-67 (2001).

146. Sciaretta, S. \& Sadoshima, J. New insights into the molecular phenotype of eccentric hypertrophy. J Mol Cell Cariol 49, 153-156 (2010).

147. Nakaoka, Y. et al. SHP2 mediates gp130-dependent cardiomyocyte hypertrophy via negative regulation of skeletal alpha-actin gene. Journal of molecular and cellular cardiology 49, 157-64 (2010).

148. Langenickel, T. et al. Differential regulation of cardiac ANP and BNP mRNA in different stages of experimental heart failure Differential regulation of cardiac ANP and BNP mRNA in different stages of experimental heart failure. Am J Physiol Heart Circ Physiol 278, H1500-06 (2000).

149. Gardner, D. Natriuretic peptides: markers or modulators of cardiac hypertrophy? Trends in Endocrinology and Metabolism 14, 411-416 (2003).

150. Hunter, J.J., Tanaka, N., Rockman, H.A., Ross, J. \& Chien, K.R. Ventricular Expression of a MLC-2vras Fusion Gene Induces Cardiac Hypertrophy and Selective Diastolic Dysfunction in Transgenic Mice *. Biochemistry 270, 23173-23178 (1995).

151. Sala, V. et al. Signaling to cardiac hypertrophy: insights from human and mouse RASopathies. Molecular medicine (Cambridge, Mass.) 18, 938-47 (2012).

152. Fujio, Y., Nguyen, T., Wencker, D., Kitsis, R.N. \& Walsh, K. Akt Promotes Survival of Cardiomyocytes In Vitro and Protects Against Ischemia-Reperfusion Injury in Mouse Heart. Circulation 101, 660-667 (2000). 
Curriculum Vitae

$\sim 192 \sim$ 


\title{
Karen A. Ryall
}

\section{EDUCATION}

\author{
Email Ryall.karen@gmail.com
}

Ph.D., Biomedical Engineering University of Virginia, Charlottesville, VA Expected May 2014

Thesis: Systems analysis of the cardiac hypertrophy signaling network.

B.S.E., Bioengineering University of Pennsylvania, Philadelphia, PA May 2009

GPA: 3.7, Minor: Engineering Entrepreneurship

\section{RESEARCH AWARDS}

Fellowships

- NSF Graduate Research Fellowship (2011-2014)

- Cardiovascular Research Center Training Grant (2010-2011)

Honors

- Biomedical Engineering Outstanding Graduate Student Award, 2014

- UVA Presidential Poster Competition Finalist, 2013

- Winner of ISHR International Poster Competition at the North American International Society for Heart Research meeting in Banff, Alberta, Canada, 2012

- UVA Engineering Graduate Research Poster Competition Finalist, 2011

- NSF Graduate Research Fellowship Honorable Mention, 2010

\section{RESEARCH EXPERIENCE}

\section{Cardiac Systems Biology Lab University of Virginia, Charlottesville, VA}

Oct. 2009-Present

- Advisor: Dr. Jeffrey Saucerman

- Developed high-throughput live-cell image acquisition and analysis platform for quantifying hypertrophy in cardiac myocytes.

- Developed integrative computational model of the cardiac hypertrophy signaling network with 106 species and 193 reactions.

- Performed a phenotypic screen of 15 hypertrophic agonists quantifying differential regulation of 5 shape features and expression of 12 genes. Follow-up experiments revealed CITED4 regulation of myocyte elongation.

- Analyzed large scale proteomics data set to identify new hypertrophy targets.

- Collaborations with Zhen Yan lab creating automated image analysis pipelines to quantitatively characterize (1) mitochondria health in muscle fibers and (2) fly heart contractions.

\section{Bioengineering Senior Design University of Pennsylvania, Philadelphia, PA}

2008-2009

- Advisor: Dr. Steven Siegel

- Optimized the release properties of a drug delivery implant for schizophrenia patients lasting up to three months.

Bioengineering REU Program University of California, San Diego, La Jolla, CA

Summer 2008

- Advisor: Dr. Andrew McCulloch

- Conducted optical mapping studies on the effects of strain on cardiomyocyte monolayers in an anisotropic stretch device. 
Injury Biomechanics Laboratory University of Pennsylvania, Philadelphia, PA

- Advisor: Dr. Susan Margulies

- Developed experimental procedure to quantify porcine brain deformation during rapid rotational accelerations.

- Collected animal behavior data from piglet head trauma studies and quantified white matter injury from brain sections.

\section{PUBLICATIONS}

- Ryall KA, Bezzerides VJ, Rosenzweig A, Saucerman JJ. Phenotypic screen quantifying differential regulation of cardiac myocyte hypertrophy identifies CITED4 regulation of myocyte elongation. J Mol Cell Cardiol. In Press.

$\checkmark$ Laker RC*, Xu P*, Ryall KA, Zhang M, Adler PN, Saucerman JJ, Yan Z. A novel MitoTimer reporter gene for mitochondrial content, structure, stress, and damage in vivo. J Biol Chem. In Press.

- Ryall KA, Saucerman JJ. Automated microscopy of cardiac myocyte hypertrophy: a case study on the role of intracellular alpha-adrenergic receptors. Methods Mol Biol. 2014. In Press.

- Ryall KA*, Holland DO*, Delaney KA, Kraeutler MJ, Parker AJ, Saucerman JJ. Network reconstruction and systems analysis of cardiac myocyte hypertrophy signaling. J Biol Chem. 2012.287(50):42259-68.

- UVA Press Release

- Ryall KA, Saucerman JJ. Automated imaging reveals a concentration-dependent delay in reversal of cardiac myocyte hypertrophy. J Mol Cell Cardiol. 2012.53(2):282-90.

- Bass GT, Ryall KA, Katikapalli A, Taylor BE, Dang ST, Acton ST, Saucerman JJ. Automated image analysis identifies signaling pathways regulating distinct signatures of cardiac myocyte hypertrophy. J Mol Cell Cardiol. 2012.52(5):923-930.

- Naim MY, Friess S, Smith C, Ralston J, Ryall K, Helfaer MA, Margulies SS. Folic acid enhances early functional recovery in a piglet model of pediatric head injury. Developmental Neuroscience.2010; 32(56):466-479.

- Ibrahim NG, Natesh R, Szczesny SE, Ryall K, Eucker SA, Coats B, and Margulies SS - In situ deformations in the immature brain during rapid rotations. J Biomech Eng. 2010: 132 (4).

- Friess SH, Ichord R, Owens K, Ralston J, Ryall K, Helfaer M, Smith C, and Margulies SS. Repeated traumatic brain injury affects composite cognitive function in piglets. Journal of Neurotrauma. 2009: 26:1111-1121.

\section{PRESENTATIONS:}

\section{Presentations at National/International Conferences}

- Ryall KA, Saucerman JJ. Phenotypic screen reveals differential regulation of cardiac myocyte hypertrophy. ISHR North American Section meeting 2013. San Diego, CA. Poster Presentation. 
Ryall KA*, Holland DO*, Delaney KA, Kraeutler MJ, Parker AJ, Saucerman JJ. Network reconstruction and systems analysis of cardiac myocyte hypertrophy signaling. ISHR North American Section meeting 2012. Banff, Alberta, Canada. Poster Presentation.

\section{- Poster competition winner}

- Ryall KA, Saucerman JJ. Automated imaging reveals a switch between reversible and irreversible cardiac myocyte hypertrophy. AHA Basic Cardiovascular Science 2011 Scientific Sessions. New Orleans, LA. Poster Presentation.

- Ryall KA, Saucerman JJ. Systems analysis of cardiac hypertrophy signaling network using automated imaging. BMES Fall 2010. Austin, TX. Platform Presentation.

- Ryall KA, Wright AT, McCulloch AD. Optical Mapping Study of the Effects of Strain on Cardiomyocyte Monolayers in Anisotropic Stretch Device. BMES Fall 2008.St. Louis, MO. Platform Presentation.

Ryall K, Ibrahim NG, Eucker S, Margulies SS. Direct Visualization of Brain Distortion During Rapid Rotational Accelerations. BMES Fall 2008. St Louis, MO. Poster Presentation.

\section{Presentations at Local Symposiums}

- Ryall KA, Saucerman JJ. "Network reconstruction and systems analysis of cardiac hypertrophy signaling." Student Summer Seminar Series. UVA Dept. of Biomedical Engineering. August 2013. Nominated Talk.

- Ryall KA, Holland DO, Delaney KA, Kraeutler MJ, Parker AJ, Saucerman JJ. Network reconstruction and systems analysis of cardiac myocyte hypertrophy signaling. UVA Presidential Research Poster Competition.

\section{- Poster competition: Biosciences and Health Finalist}

- Ryall, KA, Saucerman JJ. Automated imaging reveals a switch between reversible and irreversible cardiac myocyte hypertrophy. Biomedical Engineering Graduate Symposium. October 2012. Charlottesville, VA. Poster Presentation.

- Ryall, KA, Saucerman JJ. Automated imaging reveals a switch between reversible and irreversible cardiac myocyte hypertrophy. Robert J. Huskey Research Exhibition. March 2012. Charlottesville, VA. Poster Presentation.

- Ryall, KA, Saucerman JJ. Automated imaging reveals a switch between reversible and irreversible cardiac myocyte hypertrophy. Biomedical Engineering Graduate Symposium. September 2011. Charlottesville, VA. Poster Presentation.

- Ryall, KA, Saucerman JJ. Automated imaging reveals a switch between reversible and irreversible cardiac myocyte hypertrophy. University of Virginia Engineering Research Symposium. March 2011. Charlottesville, VA. Poster Presentation.

\section{- Finalist}

- Ryall KA, Wright AT, McCulloch AD. Optical Mapping Study of the Effects of Strain on Cardiomyocyte Monolayers in Anisotropic Stretch Device. UCSD Summer Research Symposium. La Jolla, CA. Platform Presentation. 


\section{TEACHING EXPERIENCE}

Center for the Diversity in Engineering: Introduction to Engineering Summer Program, Invited Speaker University of Virginia Charlottesville, VA

June, 2013

$\checkmark$ Lecture: "What is Biomedical Engineering" to 50 rising high school juniors and seniors

Biomedical Engineering Capstone Design, Graduate Teaching Assistantship University of Virginia Charlottesville, VA

Fall 2011-Spring 2012

- Advised 32 biomedical engineering senior design projects

- Led Design Solutions Workshops to give project feedback and give students opportunities to practice presenting

- Graded written assignments and presentations.

Center for the Diversity in Engineering: LEAD engineering summer institute, Research advisor

University of Virginia Charlottesville, VA Summer 2010

- Instructed high school students in computational modeling techniques

- Mentor for research project on modeling hypertrophy signaling network

University City High School, Guest instructor

Philadelphia, PA

2007

- Developed lesson plans and instructed an inner city high school engineering class in bioengineering topics

\section{PROFFESSIONAL EXPERIENCE}

UVA Licensing \& Ventures Group, Licensing Associate Intern

University of Virginia Charlottesville, VA

November 2011-December 2012

- Performed prior art searches to evaluate patentability of inventions from University of Virginia faculty.

- Conducted market research to identify potential licensing partners.

- Led marketing efforts directed at companies in the medical device, biotechnology, pharmaceutical, and life science products industries.

Penn Engineering Entrepreneurship minor

- Developed full business plan for biomedical device and pitched to a panel of investors.

- Investor ratings: $2^{\text {nd }}$ place overall, $1^{\text {st }}$ place product description and venture economics

- Relevant coursework: entrepreneurship I/II, engineering economics, negotiations, biotech marketplace

\section{ACADEMIC SERVICE}

- Presentation judge, UVA Biomedical Engineering Society graduate symposium

- Organizer, UVA Biomedical Engineering Society graduate symposium

- Reviewer, UVA Entrepreneurship Cup

- Invited panel speaker, Biomedical Engineering Society career panel

2011

- Coordinator, $10^{\text {th }}$ Annual Workshop on FRET Microscopy

2011

- Reviewer, SPECTRA, UVA Engineering and Science Undergraduate Research Journal

- Math and science tutor, West Philly Tutoring Project 